



\title{
Interactions with gas-rich barred galaxies
}

\author{
Dissertation \\ zur Erlangung des Doktorgrades \\ der Mathematisch-Naturwissenschaftlichen Fakultäten \\ der Georg-August-Universität zu Göttingen \\ vorgelegt von \\ Ingo Berentzen \\ aus Göttingen
}

Göttingen 2003 
D 7

Referent:

Prof. Dr. K.J. Fricke

Korreferent:

Prof. Dr. E. Athanassoula

Tag der mündlichen Prüfung: 10. Oktober 2003 
"Interacting or colliding systems form an interesting subject to study, but are not new types of galaxies. After a collision a car is a wreck, not a new type of car."

\section{de Vaucouleurs}

"...in the strange world of galaxies, two cars can indeed collide and coalesce into one limousine. “

\section{F. Schweizer}





\title{
Contents
}

\author{
1 Abstract
}

I General introduction and theory

\section{Introduction}

2.1 Barred galaxies . . . . . . . . . . . . . . . 6

2.2 Interacting galaxies . . . . . . . . . . . . . . 12

2.3 Objectives and thesis outline . . . . . . . . . . . . . . . 19

\section{Galactic dynamics}

3.1 Collisionless systems . . . . . . . . . . . . . . . . 21

3.2 Stellar orbits in barred galaxies . . . . . . . . . . . 23

\section{Numerical methods}

4.1 The algorithms . . . . . . . . . . . . . . . 33

4.2 Setting up the models . . . . . . . . . . . . . . . . . 42

\section{Results}

5 Vertical impact of small companions.

5.1 Introduction . . . . . . . . . . . . . . . . 55

5.2 Methods . . . . . . . . . . . . . . . . . . 57

5.3 Results . . . . . . . . . . . . . . . . 61

5.4 Discussion . . . . . . . . . . . . . . . . . . . . . . . . . . . . . . . . 88

5.5 Summary .......................... 92

5.6 Acknowledgments . . . . . . . . . . . . . . 93

5.7 Bibliography .......................... 94

6 The regeneration of bars by interactions

6.1 Introduction . . . . . . . . . . . . . . . . . 97

6.2 Numerical methods and initial conditions . . . . . . . . . . . 99

6.3 Isolated model with gas - model I0 . . . . . . . . . . . . . . 101 
6.4 Isolated model without gas - model I1 . . . . . . . . . . . . . . 104

6.5 Interacting models with gas . . . . . . . . . . . . . . . 106

6.6 Interacting models without gas . . . . . . . . . . . . . 111

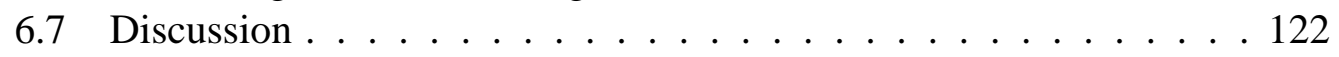

6.8 Summary . . . . . . . . . . . . . . . . . . . . 134

6.9 Acknowledgments . . . . . . . . . . . . . . . 135

6.10 Bibliography ........................ 135

7 Accuracy of pattern speed in numerical simulations.

7.1 Introduction . . . . . . . . . . . . . . . . . 137

7.2 Numerical methods and initial conditions . . . . . . . . . . . 138

7.3 Direct comparison of GRAPE-3 and -5 simulations . . . . . . . . 142

7.4 Systematic numerical tests . . . . . . . . . . . . . . . . . . . . . . . . . 147

7.5 Further numerical tests . . . . . . . . . . . . . . . . . . . . . . . . . . . . . . . . . .

7.6 Properties of the bars . . . . . . . . . . . . . . . . 154

7.7 Discussion . . . . . . . . . . . . . . . . . . . . . . 158

7.8 Summary . . . . . . . . . . . . . . . . . . . . . . 160

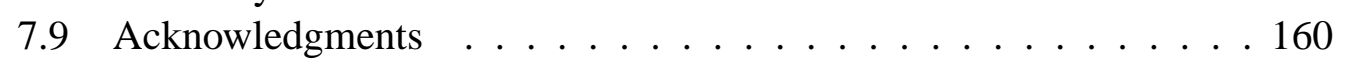

7.10 Bibliography ... . . . . . . . . . . . . . 161

7.11 Appendix: Shocks in a polytropic gas . . . . . . . . . . 162

\section{Summary and prospects}

8.1 Vertical impacts of small companions . . . . . . . . . . . . . 167

8.2 The regeneration of bars . . . . . . . . . . . . . . . . 169

8.3 Numerical accuracy of pattern speeds . . . . . . . . . . . 171

8.4 Future research . . . . . . . . . . . . . . . . . . 171

\section{Bibliography}

\section{Appendices}

\section{A Astronomical units and constants}

\section{B Documentation for the Göttinger $N$-body/SPH code}

B.1 Input files . . . . . . . . . . . . . . . . . . 185

B.2 Output files . . . . . . . . . . . . . . 185

B.3 Input parameters (FTM.NML) . . . . . . . . . . . . . 185

B.4 Model and diagnostic data . . . . . . . . . . . . . . . . . . . . . . . 188

B.5 Compile time parameters (PARAM.F) . . . . . . . . . . . 189

B.6 Model data input file format (FTM.MDL) . . . . . . . . . . . . . . . . . . . . . . . . . . . . . . . . . . .

B.7 Data Structures . . . . . . . . . . . . . . . . . . 190

B.8 Examples I . . . . . . . . . . . . . . . . . . . 191 
B.9 Examples II . . . . . . . . . . . . . . . . . . . . . 191

B.10 Flow Diagram . . . . . . . . . . . . . . . 193

C The GRAPE-system in Göttingen (GÖ-GRAPE)

D Acknowledgements

E Lebenslauf 
1. Abstract

In this work we study the effects interactions have on the evolution of gas-rich barred galaxies using $N$-body/smoothed-particle-hydrodynamics simulations.

In the first part we investigate the dynamical effects of an interaction between an initially barred galaxy and a small spherical companion. In these models the small companion passes through the disc of the larger galaxy nearly perpendicular to it's plane. The impact position and time are varied with respect to the phase of the bar and the dynamical evolution of the disc. We find that the interactions produce expanding ring structures, offset bars, spokes, and other asymmetries in the stars and gas. We describe how the evolution of the bar strength, pattern speed, and gas inflow rate are affected by the interaction. The results are compared with pure stellar simulations to assess the role played by the dissipative component on the evolution of the disc and bar during the interaction.

In the second part, we study the regeneration of stellar bars triggered by a tidal interaction, using numerical simulations of either purely stellar or stellar+gas disc galaxies. We find that interactions which are sufficiently strong to regenerate the bar in the purely stellar models do not lead to a regeneration in the dissipative models, owing to the induced gas inflow. In models in which the bar can be regenerated, we find a tight correlation between the strength and the pattern speed of the induced bar. This relation can be explained by a significant radial redistribution of angular momentum in the disc due to the interaction, similar to the processes and correlations found for isolated barred spirals. We furthermore show that the regenerated bars show the same dynamical properties as their isolated counterparts.

In the final part, we present a systematic study of the influence of numerical effects on the evolution of the pattern speed of bars in fully self-consistent simulations. We show that the evolution of the pattern speed is very sensitive to both the intrinsic numerical noise of the model, as well as to the numerical accuracy in the force calculation. Owing to the superposition of these effects the pattern speed $\Omega_{\mathrm{p}}$ shows an uncertainty of roughly 13 per cent. We conclude that large particle numbers and high force accuracy are required for a robust determination of $\Omega_{\mathrm{p}}$. 


\section{General introduction and theory}





\section{Introduction}

Galaxies are assumed to be the basic building blocks of the large-scale visible structure in our Universe. A typical galaxy consists of a baryonic component of stars $\left(10^{10}-10^{12}\right)$ and gas mixed with dust (up to some 30 per cent), and a large fraction by mass of non-baryonic material or dark matter. The morphological appearance of galaxies is varied and a classification scheme had been first introduced by Edwin P. Hubble (1926), which is known as the Hubble sequence of galaxies. Hubble divided the galaxies, based on galaxy images on photographic plates, into basically three main classes: elliptical (E), spiral (S) and irregular (Irr) galaxies. The spirals are subdivided into two families, the normal spirals (SA) and the barred spirals (SB). The classical representation of the Hubble sequence, which is, because of its appearance, called the tuning-fork diagram, is shown in Fig. 2.1. Elliptical galaxies contain almost no gas and are dominated by an older stellar population, giving them a reddish appearance. S0 galaxies represent a transitional type between elliptical and spiral galaxies. Spiral galaxies are in contrast to ellipticals rotationally supported systems, with a relatively thin stellar disc, typically less and 10 per cent of their diameters. The gas content of the galaxies increases in the Hubble sequence from left to right from almost zero to 20-30 per cent of the baryonic mass. Furthermore, the central bulge component decreases. Historically, the galaxies are called late- and early-type galaxies.

Observations only provide a snapshot of galaxies and thus it is not possible for us get direct information about their dynamical and morphological evolution in time. Numerical simulations of galaxies, however, provide us a tool to follow their evolution. It is now clear that the morphological and dynamical evolution of disc galaxies is driven through both internal and external processes. Instabilities in the disc often give rise to the formation of bars. Because of their striking morphology and dynamical importance on the evolution of the galactic discs, bars have been the subject of intensive study.

Galaxies are in general not isolated objects, but often appear in groups or clusters. Also many field galaxies have a number of small, i.e. less massive, companion galaxies. Interactions between galaxies are therefore frequent events, encounters with the smaller companions are with equally or more massive neighbouring galaxies.

In this work we study several aspects of interactions with gas-rich barred galaxies by means of numerical simulations. We describe the morphological and dynamical evolution of the bar and the disc. 


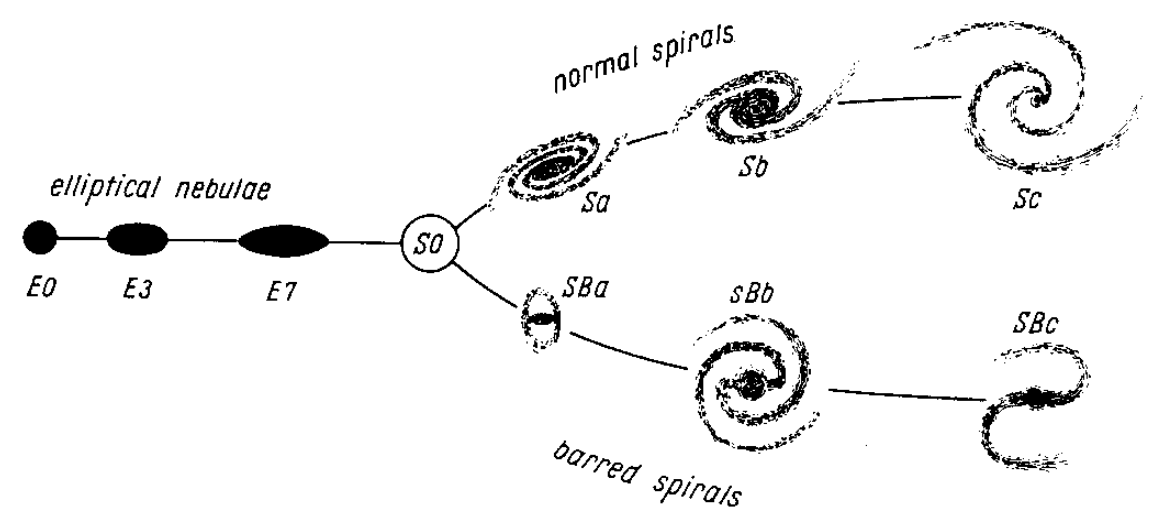

Figure 2.1: Tuning-fork diagram of the Hubble sequence of galaxies (Hubble 1926).

\subsection{Barred galaxies}

Stellar bars in disc galaxies are a common feature. The nearest barred galaxy although difficult to observe directly - is our own, the Milky Way (see, for example, de Vaucouleurs 1964; Blitz \& Spergel 1991; Kuijken 1996; Gerhard 1996, and references therein). In Fig. 2.2 we show a striking example of a barred galaxy. The large-scale bar consists predominantly of older stars, giving it a reddish colour. Some light of the bar is obscured by the off-set leading dust-lanes across the bar. Connected to the end of the bar are two long spiral arms. Both the bar and the spiral arms rotate clockwise in this image.

As shown in Fig. 2.3 (upper panel), barred galaxies amount to more than one third of the catalogued disc galaxies in optical wavelengths (see, for example, de Vaucouleurs 1963; Sellwood \& Wilkinson 1993). With a growing number of available near-infrared (hereafter NIR) observations (see, for example, Pompea \& Rieke 1990; Rix 1993; Rix \& Rieke 1993; Seigar \& James 1998; Eskridge et al. 2000; etc.), it has been confirmed that the fraction of barred galaxies is even higher, i.e. up to roughly two thirds (Eskridge et al. 2000; see also Fig. 2.3, lower panel). As can be seen from Fig. 2.3 the fraction of barred galaxies seems to be independent

\footnotetext{
${ }^{1}$ Credit European Southern Observatory (ESO).
} 


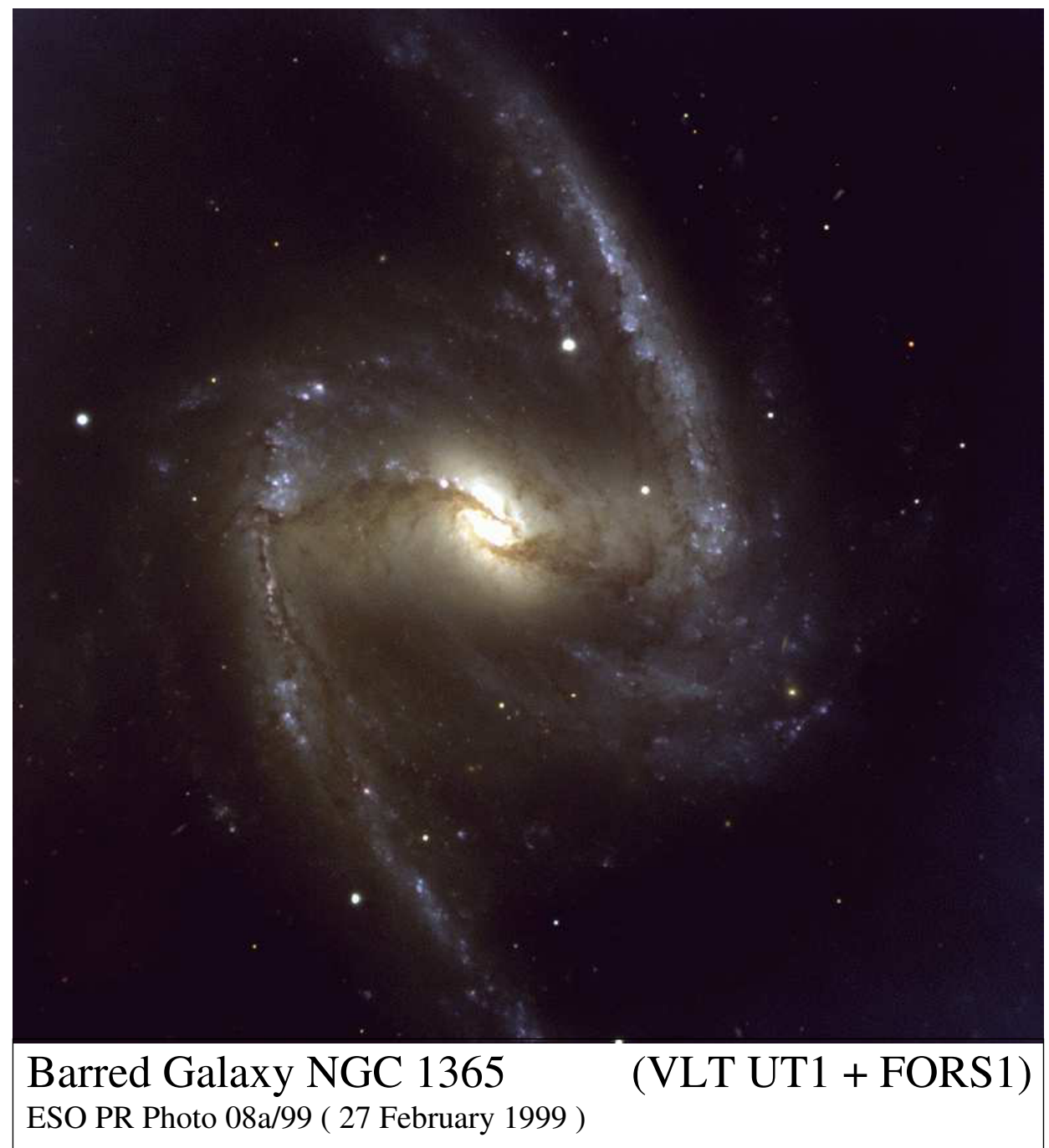

Figure 2.2: Barred spiral NGC 1365 (Credit European Southern Observatory ESO). This galaxy is the largest spiral in the Fornax cluster of galaxies.

of the galaxies Hubble-type. The properties of the bars, however, vary with respect to the Hubble-type. Elmegreen \& Elmegreen (1985) found that bars in early-type galaxies have uniform luminosity profiles and are longer than the ones in late-type galaxies, which have exponential-like density profiles. Furthermore, Athanassoula \& Martinet (1980) and Martin (1995) found a correlation between the length of the bar and the size of the bulge component, in the sense that less massive bulges allow larger bars.

Because of their importance to the dynamical evolution of disc galaxies, barred galaxies have been extensively studied in the past, both from the observational 


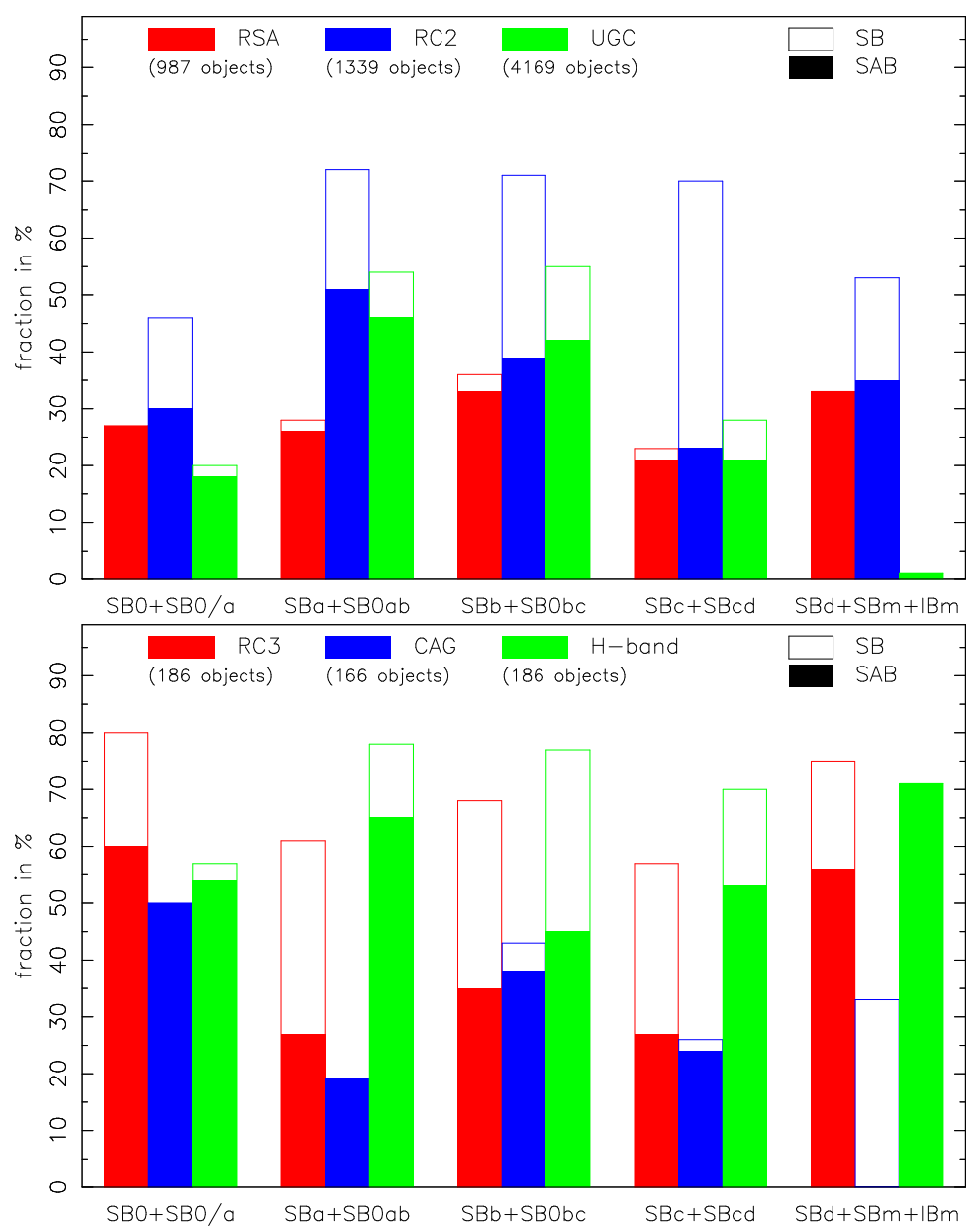

Figure 2.3: Bar-fraction as a function of morphological type. The upper panel shows the fraction of bars in galaxies from different catalogues (Sellwood \& Wilkinson 1993). The lower panel shows the fraction of bars found in a sample of galaxies observed in the H-band in comparison with the catalogued classification (Eskridge et al. 2000).

and theoretical side. It has been established, that the life of a bar can be divided into several episodes (see, for example, the review by Friedli 1999): its formation (Sec. 2.1.1), evolution (Sec. 2.1.2), dissolution (Sec. 2.1.3) and maybe its regeneration (Sec. 2.1.4).

\subsubsection{Bar formation}

Two mechanisms for the formation of bars in disc galaxies are being widely accepted at present. Bar formation can occur, as shown in many numerical simula- 
tions, spontaneously by a global instability in cold, rotationally supported stellar discs (see, for example, Miller et al. 1970; Hohl 1971; Ostriker \& Peebles 1973; Sellwood 1981; Athanassoula \& Sellwood 1986; etc.). The bars can be considered as long-living modes in the disc, caused by the formation of a standing wave by superposition of leading and trailing waves. As outlined by Toomre (1981) for spiral density waves, the bar mode can grow through swing amplification.

It was found early on that the dark matter halo of a galaxy has a stabilizing influence on the disc and the formation of bars (Ostriker \& Peebles 1973). These authors postulated the condition for a rotating stellar system to be stable against the growth of the bar mode, known as the Ostriker-Peebles criterion, as $t \equiv T_{\text {rot }} /|W| \geq$ 0.14 , where $T_{\text {rot }}$ and $W$ are the rotational kinetic energy and the gravitational energy of the system, respectively. As reported only recently, however, even in massive halos the bar growth can be stimulated in the disc due to the destabilising influence of resonant stars in the halo (Athanassoula 2002).

The second mechanism, also confirmed in numerous $N$-body simulations, is the formation of tidally induced bars, triggered by interactions with neighbouring galaxies (see, for example, Byrd et al. 1986; Noguchi 1987, 1988, 1996; Barnes \& Hernquist 1991; Salo 1991; Miwa \& Noguchi 1998; etc.). In this section we will concentrate on the description of bars in isolated disc galaxies, and will come back to tidally induced bars and their properties in Sec. 2.2.

\subsubsection{Evolution of bars}

Once a stellar bar has formed, it changes both the kinematics and the mass distribution within the disc and undergoes further evolution itself, which often ends in a significant weakening or sometimes even destruction of the bar.

The presence of a rotating bar often gives rise to dynamical resonances in the disc, which can be associated with inner and outer rings as found in many barred spirals (see, for example, Schwarz 1979, 1981, 1984a, 1984b, 1984c; Buta 1983, 1984; Combes \& Gerin 1985; etc.). In these works it has been shown that gaseous rings can arise around the Lindblad resonances owing to the perturbation of the bar. The most fundamental parameter describing the dynamics of the bar and the corresponding dynamical resonances is the pattern speed $\Omega_{\mathrm{p}}$, i.e. the angular frequency with which the bar rotates in the disc. The direct measurement of $\Omega_{\mathrm{p}}$ from observations, however, remains a problematic.

One way to measure $\Omega_{\mathrm{p}}$ from observations is by the Tremaine-Weinberg method (Tremaine \& Weinberg 1984a). This method is based on the continuity equation of a tracer population. However, this method is strongly sensitive to errors in the position angle of the disc (Debattista 2003). Another way is to locate the radius of corotation in the disc as derived from the non-circular motion in the disc owing to the bar and spiral structure (Canzian 1993). Furthermore, if the potential of the galaxy can be derived, for instance from near-infrared data, one can use numerical hydrodynamical simulations (see, for example, Sempere et al. 1995a, 1995b; 
Lindblad et al. 1996; Lindblad \& Kristen 1996; Laine et al. 1998; etc.) to find $\Omega_{\mathrm{p}}$. In this method the pattern speed is a free model parameter and can be varied until the observed gas-flow patterns is reproduced sufficiently well. As shown by Patsis \& Athanassoula (2000), the morphology and the inflow are dependent on the hydrodynamical and numerical parameters used in the models.

Numerical simulations of barred galaxies indicate that the pattern speed of the bar does not remain constant during its evolution. The bar can efficiently redistribute and exchange angular momentum with the disc and the spherical galactic components (see, for example, Sellwood 1980, 1981; Weinberg \& Tremaine 1983; Weinberg 1985; Little \& Carlberg 1991a, 1991b; Debattista \& Sellwood 1998, 2000; Athanassoula 1996a). Athanassoula (2003) argued that the evolution of bars in isolated disc galaxies is driven by this redistribution of angular momentum. Disc galaxies strive to transfer angular momentum outwards (Lynden-Bell \& Kalnajs 1972). Disc material in the inner disc, being at resonance with the bar, emits angular momentum to resonant material in the outer disc or to the spheroidal components like the halo and bulge (Athanassoula 2002). The corotation radius of the bar - i.e. the radius at which the disc material rotates with the same angular frequency as the bar - divides the regions of emitters and absorbers. The size and the pattern speed of the bar responds to the angular momentum exchange such as to keep an equilibrium between the emitters and absorbers. These recent results argue strongly that the strength and pattern speed of the bar is determined by the amount of angular momentum exchanged. The bar's slowdown rate found in numerical simulations depends on the relative halo mass and on the velocity dispersion of both the disc and the halo. Furthermore the author has shown that bar growth in the disc can be stimulated even in massive halos due to the destabilising influence of resonant stars in the halo (Athanassoula 2002).

If the bar in the disc becomes sufficiently massive and long, the so-called firehose (or buckling/bending) instability out of the equatorial disc plane may occur (see, for example, Hunter \& Toomre 1969; Combes et al. 1990; Pfenniger \& Friedli 1991; Raha et al. 1991; etc.). After this vertical instability sets in, the disc settles with an increased thickness and vertical velocity dispersion. During and after this phase the bar appears boxy or peanut-shaped when viewed edge-on. Thus this instability may be associated with boxy/peanut-shaped bulges (see, for example, Bureau \& Freeman 1997 and references therein). The fire-hose instability is found to be prominent especially in pure $N$-body simulations. These simulations show that if the instability is very rapid the bar can be destroyed. On the other hand the instability seems to be greatly damped out in models of gas-rich barred discs (Berentzen et al. 1998) owing to the stabilizing influence of the dissipative component.

\section{Influence of the gas}

Another factor influencing the evolution of bars is the interstellar medium in the disc. Although the gas usually contributes only a small fraction to the mass of a 
galaxy, it can significantly influence it's evolution (see, for example, Shlosman \& Noguchi 1993). Many numerical simulations that have included a dissipative disc component have emphasized the importance of the gas in isolated barred galaxies (see, for example, Friedli \& Benz 1993; Heller \& Shlosman 1994; Berentzen et al. 1998). The dynamics of the gas in barred galaxies can be understood by the orbital structure of the disc. A clear introduction to this subject has been given by Prendergast (1983). The gas streamlines tend to follow periodic orbits in the potential of the galaxy. In contrast to stellar trajectories, however, the streamlines in the gas cannot cross. In the case of intersecting orbits, the gas dissipates energy by the way of shocks and thus change orbits (e.g., Sanders et al. 1983). Many barred galaxies of types SBb to SBc show dust lanes in the bar, predominantly on its leading side. Such dust lanes are associated with the shocks in the gas flow, as first claimed by Prendergast (1962, unpublished). Numerical simulations have shown that the shape of the dust lanes reveals information about the internal dynamics of the bar and disc (Athanassoula 1992b).

Owing to the gravitational torques of the bar, gas is driven towards the centre of the galaxy, which might then be accompanied by a central starburst (Heller \& Shlosman 1994) and formation/fuelling of an active galactic nucleus (Shlosman, Frank \& Begelman 1989; Shlosman, Begelman \& Frank 1990). Recent NIR-observations (Pèrez-Ramírez et al. 2000) have also shown a relation between the circumnuclear star formation in barred galaxies and the circumnuclear spiral or ring structure present in these galaxies. The large-scale redistribution of disc material owing to the bar also affects chemical abundance gradients across the disc (see, for example, Edmunds \& Roy 1993; Martin \& Roy 1992; Martin \& Friedli 1999; etc.)

Fully self-consistent numerical simulations including a gaseous disc component have shown that a substantial gas inflow can significantly weaken or even destroy the bar (see, for example, Friedli \& Benz 1993; Berentzen et al. 1998). The central mass concentrations and the times-scales found in these simulations, however, might be too high and too short, respectively, to be in agreement with observations (e.g. Sakamoto et al. 1991) and the relatively high fraction of barred galaxies observed. Furthermore, the fraction of bars seems to be independent of the galaxies morphology (see Sec. 2.1), while early-types are known to have considerably less gas.

\subsubsection{Bar dissolution}

Once they have formed, bars do not stay eternally in the disc, but may instead weaken or dissolve over time. The dissolution of bars can be driven both by internal and external processes, though the basic mechanism in both cases is often a significant change of the central mass concentration in the galaxy. The most efficient mechanism to transfer angular momentum within the disc and induce gas inflow are the gravitational torques from a global non-axisymmetric perturbation of the potential such as a from a bar or interaction. Several numerical simulations 
have shown that the growth of a central mass concentration affects the bar structure and often leads to its gradual dissolution (Kormendy 1982; Hasan \& Norman 1990; Friedli \& Pfenniger 1991; Friedli \& Benz 1993; Hasan et al. 1993; Norman et al. 1996; Berentzen et al. 1998). Friedli (1999) proposed a critical mass ratio $\gamma_{\text {anni }} \equiv M_{\text {centre }} / M_{\text {total }} \approx 0.1 R+0.02$, where $R$ is the radius considered, as a rough threshold to annihilate the bar. Besides the growth of central mass concentration, the formation of massive nuclear rings (Heller \& Shlosman 1996), as well as the onset of a very rapid fire-hose instability tend to significantly weaken or even dissolve the bar.

\subsubsection{Bar regeneration}

The regeneration of the stellar bar, i.e. a second bar-formation episode during the lifetime of the disc, has been suggested as a possible mechanism to explain the observed number of barred galaxies. One possible scenario for this, as suggested by Sellwood \& Moore (1999), could be the accretion of gas-rich companion galaxies or freshly infalling gas, which, by adding colder material to the disc, may cool it sufficiently and allow the generation of a new bar. The difficulty with this approach is that the dissolution of a bar is accompanied by an increase in the stellar velocity dispersion, which further stabilizes the disc against future bar formation. Bournaud $\&$ Combes (2002) studied the effects of gas accretion on the evolution of spiral galaxies by means of $N$-body simulations including star-formation and feedback processes. From their models they indeed found recurrent episodes of bar formation and dissolution.

Another scenario could be the regeneration of a previously dissolved bar by a sufficiently strong tidal encounter with a neighbouring galaxy. While many studies have looked into the formation of bars by tidal interactions (see, for example, Byrd et al. 1986; Noguchi 1987, 1988; Gerin, Combes \& Athanassoula 1990; Barnes \& Hernquist 1991; Salo 1991; Miwa \& Noguchi 1998; etc.), the regeneration of bars by such a mechanism has not to our knowledge been previously examined. We will describe the effects of interactions on the evolution of disc galaxies in more detail in the following section.

\subsection{Interacting galaxies}

Interactions between galaxies play a fundamental role in the formation and evolution of galaxies in current hierarchical cosmological models. Deep-field observations, both ground-based and HST, reveal that interactions and mergers between galaxies were much more common in the past. Even the Milky Way will most likely collide with our neighbour M31 (i.e. the Andromeda galaxy) in some $10^{10}$ years. Furthermore, most disc galaxies have less massive companion galaxies. It is clear that interactions between galaxies are frequent enough to be a major factor influenc- 
ing their dynamical evolution. For a review on interacting galaxies see, for example, Barnes \& Hernquist (1992) and Struck (1999).

The term interaction usually refers to the gravitational interaction between the affected galaxies. Various signs of interactions have been found in the catalogues of peculiar galaxies and galaxies pairs (see, for example, Vorontsov-Velyaminov 1959; Vorontsov-Velyaminov \& Krasnogorskaya 1961; Zwicky 1961; Arp 1966; Vorontsov-Velyaminov 1977; etc.). Many of these galaxies show bridges and tails, which are now known to be of tidal origin. Furthermore, some of the galaxies are in the process of merging, i.e. forming a single bound system. That the observed features are indeed of tidal origin has been confirmed by Holmberg (1941) using sophisticated laboratory models of galaxy encounters, as well as by early numerical simulations using test particles by Pfleiderer \& Siedentopf (1961) and Pfleiderer (1963). In the ground-breaking work of Toomre \& Toomre (1972) finally demonstrated, based on restricted $N$-body simulations, that gravity alone could account for the formation of such observed structures. The authors presented also a detailed modelling of four particularly well-known pairs of interacting galaxies. It has also been suggested that the gravitational interaction between two self-gravitating bodies can give rise to the formation of spiral structure - in a broader context though for the first time by Chamberlin (1901).

The blue colors, an indicator for ongoing star-formation, found in interacting galaxies (Holmberg 1958) can be explained by bursts of star-formation with ages and durations of the order some $10^{7}-10^{8} \mathrm{yr}$ (Larson \& Tinsley 1978). Furthermore, observations indicate a clear link between tidal interactions and nuclear activity (Hummel 1981; Kennicutt \& Keel 1984; Keel et al. 1985), as well as a higher frequency of Seyfert activity (Dahari 1984). Strong interactions in gas-rich systems can transport large amounts of interstellar gas to the centres of the interaction remnants and thus trigger nuclear starbursts or nonthermal nuclear activity (e.g., see reviews by Heckman 1990, 1994; Sanders 1992).

During the encounter between two galaxies, they may suffer a a gradual loss of their orbital energy owing to dynamical friction (see, for example, Chandrasekhar 1942; Tremaine \& Weinberg 1984b). Owing to this orbital decay the interaction between the galaxies finally leads to merging of the two systems. Two well-known examples of nearly equal mass disc galaxies, which are still in the merging process are NGC 4038/4039 (The Antennae) and NGC 4676 (The Mice). The outcome of a merger strongly depends on the mass ratio of the two participants. In general two different types of mergers are distinguished. Mergers of comparable mass spirals, so called major mergers, are the most striking example of galaxy collisions. Toomre \& Toomre (1972) and Toomre (1977) argued that major merger events are the dominant process by which early-type galaxies form. There is growing evidence from observations of fine structure and indicators of past starbursts in elliptical galaxies (see, for example, the reviews by Schweizer 1998 and Kennicutt 1998, and references therein), as well as from numerical simulations (see, for example, Barnes 1988, 1992; Hernquist 1992, 1993; Barnes \& Hernquist 1992, 1996, 1998; Barnes 
1998 and references therein), that indicate at least some elliptical galaxies have formed by a merger of two spiral galaxies.

Major mergers are, however, less common than minor mergers, i.e. the accretion of satellites or dwarfs on disc galaxies. Minor merger are in general less violent and numerical simulations have shown that they can drive the morphological evolution of the disc without actually destroying it (see, for example, Quinn \& Goodman 1986; Quinn et al. 1993). For instance, minor mergers may trigger starbursts (Mihos \& Hernquist 1994a), excite nuclear activity (Hernquist \& Mihos 1995), lead to the formation of warps and bars (Quinn et al. 1993; Laine \& Heller 1999) and to the dynamical heating of the disc (see, for example, Toth \& Ostriker 1992; Schwarzkopf \& Dettmar 2000; etc.).

In the cases above the interaction leads to galaxy mergers. However, if the encounter of the two galaxies is fast enough the galaxies will remain unbound and a interaction can occur with out a merger. In this work we focus on less violent interactions. In general interactions can be distinguished between collisions (Sec. 2.2.1) and tidal encounters of galaxies (Sec. 2.2.2).

\subsubsection{Galaxy collisions}

An only recently recognised important external agent for driving galaxy evolution is the interaction with small companions. Statistical surveys by Zaritsky et al. (1993, 1997) have shown that the average number of companions for field spiral galaxies has a lower limit of $1.2 \pm 0.3$ (see also Fig. 2.4). Thus we expect interactions between a disc galaxy (or host) and less-massive galaxies (or companion) to be a frequent phenomenon during the lifetime of a galaxy.

Particularly spectacular, though probably not the most common case, are interactions in which the companion galaxy passes nearly perpendicular through the equatorial plane of the host's disc, which often gives rise to the formation of impressive ring-like structures. A most striking example of such a collisional ring galaxy is the Cartwheel-Galaxy (AM 0644-741; see Fig. $2.5^{2}$ ). For recent $N$-body modelling of the Cartwheel galaxy, see for example Horellou \& Combes (Horellou \& Combes 2001) and Villa, Athanassoula \& Bosma (Villa et al. 2002). These collisionally induced rings have a different physical origin and dynamical properties than the resonance rings often found in isolated discs.

The formation of collisionally induced rings can be described by the impulse approximation (see, for example, Binney \& Tremaine 1987). The passage of the companion through the disc excites both radial and vertical oscillations in the disc. The induced radial oscillatory motion of the stars and the gas produces expanding density ring-waves (see, for example, Lynds \& Toomre 1976) centred on the impact

\footnotetext{
${ }^{2}$ Based on observations with the NASA/ESA Hubble Space Telescope (HST), obtained from the Space Telescope Science Institute, which is operated by the Association of Universities for Research in Astronomy, Inc., under NASA contract NAS 5-26555. These observations are associated with Photo Release No. STScI-PRC 95-02.
} 


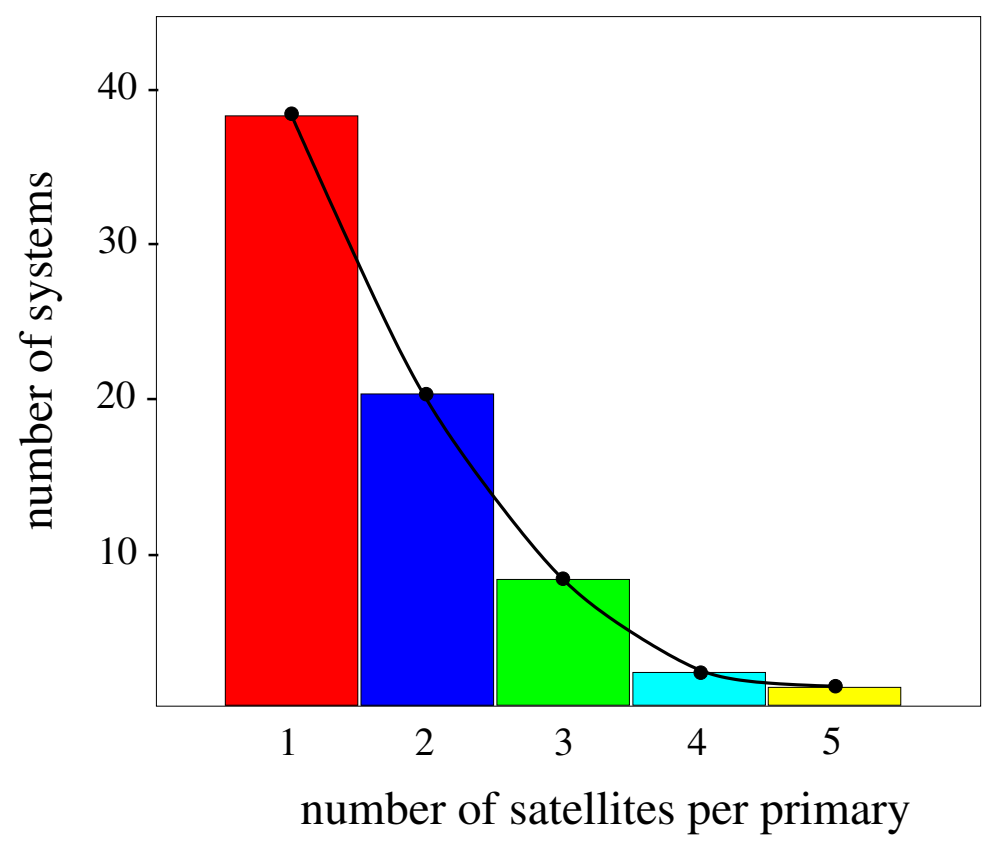

Figure 2.4: Statistic of satellites per field galaxy (Zaritzky et al. 1997)

position, while the vertical oscillations lead to an increase in the vertical velocity dispersion and a significant thickening of the disc. As noted by Athanassoula, Puerari \& Bosma (1997), spokes form only between the first and the second ring, the presence of the latter being necessary. The properties of those spokes in collisional ring galaxies are discussed in detail by Hernquist \& Weil (1993).

Impacts along trajectories 30 degrees (and sometimes higher) from the disc normal still yield ring shaped features (Lynds \& Toomre 1976). The effects of oblique impacts have been studied by Toomre (1978), Athanassoula (1999) and Athanassoula et al. (1997). In the latter work, concentrating on the formation of collisionally induced rings, the authors present purely collisionless simulations of off-centred impacts of a sufficiently massive companion hitting the inner parts of a barred disc galaxy. They have shown that the impact can displace the bar to one side, causing asymmetries and the formation of rings. So far, the simulations of such collisions involving barred galaxies have been purely stellar and not much is known about the influence of the dissipative component in such encounters.

\subsubsection{Tidal encounters}

Since the pioneering work of Toomre \& Toomre (1972) much effort has been spent to investigate galaxy-galaxy interactions by means of numerical simulations (e.g. see the review Barnes \& Hernquist 1992, and references therein). As mentioned 


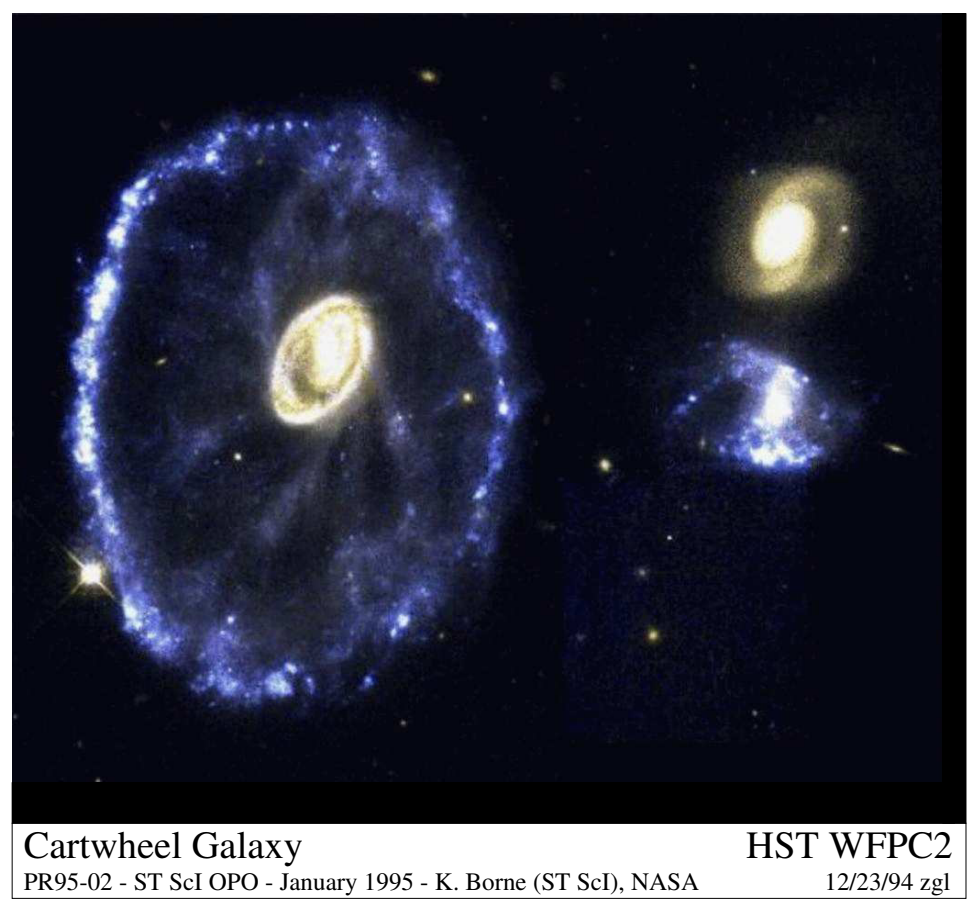

Figure 2.5: HST observation of the collisional double-ring galaxy AM 0644-741 (or Cartwheel galaxy). On the right hand side are the two companion galaxies and interaction candidates.

before, these simulations have shown that gravitational forces alone are able to reproduce many of the observed features, such as tidal arms, in great detail.

Toomre \& Toomre (1972) argued that the bridges and tails often found in galaxy pairs are tidal relicts of close encounters. The authors studied such interactions and the formation of tidal arms by means of restricted $N$-body models. They found the strongest effect, when the encounter is prograde with respect to the discs rotation and the orbits are close to the equatorial plane of the disc, with inclinations less then $30^{\circ}$. Owing to the gravitational torques the angular momentum of the material in the outer disc increases and is pulled out of the galaxy, forming impressive tidal arms. Only recently, interest in the study of tidal arms has been revived, as they are suspected to be the birthplace of dwarf galaxies, the so-called tidal dwarfs. Such a mechanism for the formation of tidal dwarf galaxies was first proposed by Schweizer (1978) for the southern tail of the Antennae galaxies (NGC 4038/39). Further support for this scenario has been given by, for example, Mirabel et al. (1991, 1992) in the Superantennae (Am-1925-724) and Weilbacher et al. (2000, 2003; see also Fig. 2.6). 


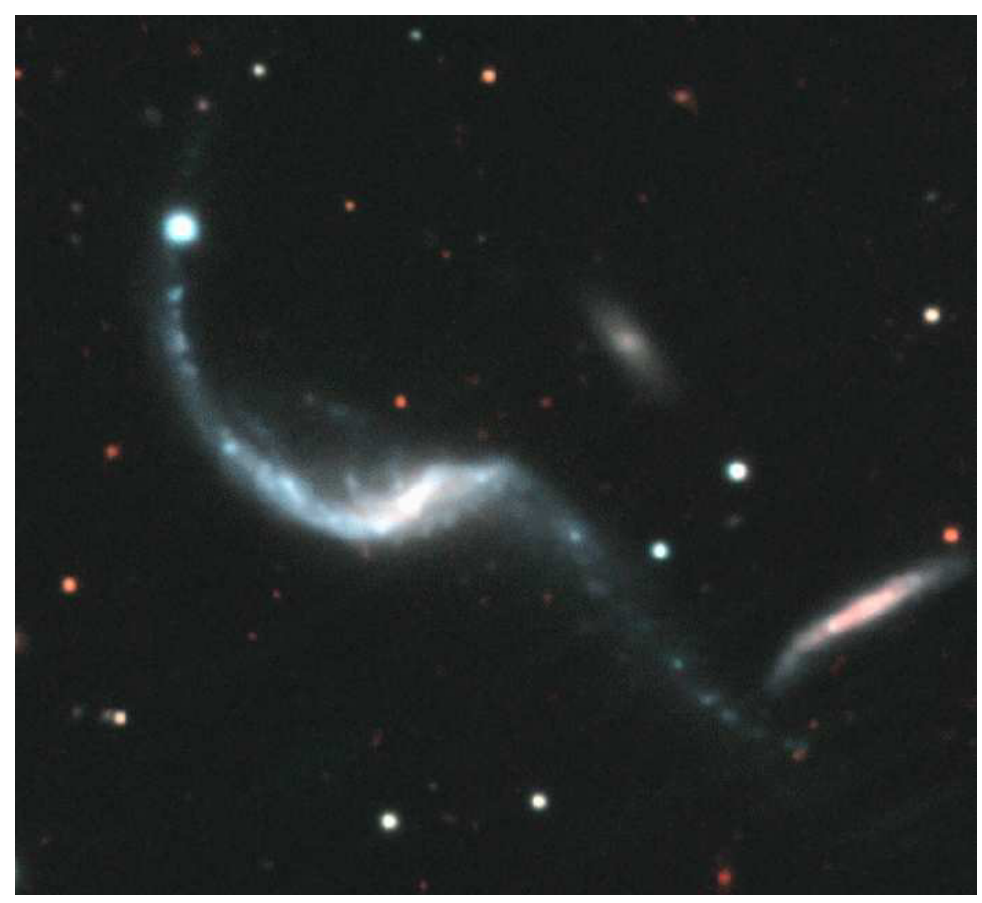

Figure 2.6: Interacting galaxy AM 1353-272 (The Dentist's Chair). Composite $B+V+$ NIR true color image from NTT-SUSI (Weilbacher et al. 2000) and SOFI (Weilbacher in prep.)

\section{Interactions and bars}

Numerous $N$-body simulations have demonstrated that tidal interactions with neighbouring galaxies can give rise to the formation of stellar bars (see, for example, Byrd et al. 1986; Noguchi 1987, 1988; Gerin, Combes \& Athanassoula 1990; Barnes \& Hernquist 1991; Salo 1991; Miwa \& Noguchi 1998; etc.). These tidally induced bars form more rapidly than there isolated counterparts and effectively funnel gas into the central disc region (Noguchi 1987, 1988), thus providing a source of fuel for nuclear starbursts and active galactic nuclei.

Based on 2D $N$-body simulations, Salo (1991) has used the Dahari index (Dahari 1984) as a quantitative measure for tidally induced bar formation and found a minimum value of $Q_{\mathrm{D}}$, depending on the specific host galaxy, above which a bar is formed by the interaction. The specific value of $Q_{\mathrm{D}}$ as a criterium for induced bar formation, however, depends strongly on the central mass concentration of the host galaxy. Elmegreen et al. (1991) defined a more advanced parameter for which a limiting value was also found.

Elmegreen \& Elmegreen (1985) found two different types of major axis surface density profiles of bars, exponential and the flat, corresponding to bars in late- and 
early-type galaxies. Noguchi (1996) argued that this distinction could be due to the fact that the first type is found in spontaneous bars and the second in driven ones. To distinguish between the two bar forming mechanisms, Miwa \& Noguchi (1998) compared the properties of spontaneously formed and tidally induced bars by means of $N$-body simulations and argued that tidally induced bars are slow rotators, while the spontaneously formed bars usually have higher pattern speeds.

Gerin, Combes \& Athanassoula (1990) have performed both 2D and 3D Nbody simulations to study the response of a bar formed in a initially globally unstable disc (Ostriker \& Peebles 1973), to a tidal encounter with a spherical perturber. They studied the effect of close encounters, both in and out of the plane, on trajectories with pericentres outside the disc. This work has been continued by Sundin \& Sundelius (1991) and Sundin, Donner \& Sundelius (1993), who have studied the response of a bar using 2D $N$-body simulations, which had been induced by a satellite on a planar parabolic orbit. In these works the change in pattern speed and angular momentum distribution and resonances in the disc have been investigated for different orbits and masses of the perturber. Depending on the initial conditions the strength of the bar transiently enhanced or decreased owing to the tidal interaction. This is accompanied by an increase or decrease of the bar's pattern speed. 


\subsection{Objectives and thesis outline}

The aim of this work is to study the morphological and dynamical evolution of barred galaxies undergoing interactions with companion galaxies. In particular we take the gaseous component into account, which is known to play a significant role in the evolution of disc galaxies. We use a fully self-consistent 3D $N$-body/smoothed-particle-hydrodynamics algorithm to evolve both the stellar and gaseous galaxy components.

Firstly, we briefly summarize in Sec 3 the basic theory of stellar orbits and galactic dynamics in respect to the evolution of barred galaxies. We then describe in Sec. 4 the algorithm used for the numerical simulations presented in this work and provide a description of the set-up procedure for the initial conditions of our models.

In Sec. 5 we focus upon the evolution of a gas-rich barred disc galaxies, which are perturbed by the impact of a less massive spherical companion galaxy. In these simulations the companion galaxy passes through the disc of the larger galaxy nearly perpendicular to the equatorial plane of the disc. The impact position and time are varied with respect to the bar and evolutionary phase of the isolated barred galaxy. The mass of the companion galaxy has been chosen such that the interaction can be considered as a perturbation to the bar and disc. The set of encounter parameters has been taken from the simulations of Athanassoula, Puerari \& Bosma (1997) and chosen to produce considerable effects, like asymmetries, off-centred bars and formation of rings.

In Sec. 6 we study the regeneration process of stellar bars triggered by a tidal interaction, providing a comparison between purely stellar and stellar+gas simulations. We describe the conditions necessary to regenerate the bar in the disc. We also compare the properties of tidally regenerated bars to those formed by the spontaneous bar instability in isolated disc. In addition, we describe how the radial redistribution of angular momentum due to the interactions affects the regeneration process.

In Sec. 7 we present a first systematic study of the influence of different numerical effects on the accuracy of pattern speeds determined from numerical simulations. The simulations presented in this sections are carried out on different hardware, i.e. GRAPE-3 and GRAPE-5, as well as different numerical schemes, like direct summation and treecode methods.

Finally, in Sec. 8 we give a brief summary of the main results of this work and give an outlook on future research in Sec. 8.4. In the Appendix the astronomical constants frequently used in this work are given, along with a detailed description of the $N$-body/SPH code (Sec. B) and GRAPE-System (Sec. C) used for the simulations. 


\section{Galactic dynamics}

\subsection{Collisionless systems}

Galaxies can be considered as dynamical systems, which evolve secularly on cosmological time-scales. Before giving a brief introduction into the field of galactic dynamics, we first give some estimates of characteristic time-scales related to the evolution of stellar systems in general. The crossing time $t_{\mathrm{c}}$ is defined by the time that a star with a mean velocity $v$ needs to cross a system with a linear size $L$ :

$$
t_{\mathrm{c}}=\frac{L}{v}
$$

For a typical disc galaxy with a diameter of roughly $L=30 \mathrm{kpc}$ and a mean velocity of $v=250 \mathrm{~km} \mathrm{~s}^{-1}$, the crossing time is of the order of some $10^{8} \mathrm{yr}$. When moving through the galaxy, however, the orbit of a star gets deflected from its original path by successive encounters with other stars. The relaxation time $t_{\mathrm{r}}$ in a stellar system is defined as the time over which these cumulative effects of stellar encounters become comparable to the stars initial velocity. In terms of the crossing times $t_{\mathrm{c}}$, the relaxation time is given by (see, for example, Binney \& Tremaine 1987):

$$
t_{\mathrm{r}} \approx \frac{N}{8 \ln N} t_{\mathrm{c}}
$$

where $N$ is the number of stars in the system. The relaxation time increases compared to the crossing time with increasing $N$. For a typical galaxy with $N \approx 10^{11}$ stars the relaxation time is roughly $t_{\mathrm{r}} \approx 6 \times 10^{17} \mathrm{yr}$. This means that the relaxation time in stellar systems with large $N$ is much longer than the crossing time. Since galaxies are only some 100 crossing times old, however, cumulative effects of stellar encounters or collisions are not significant in the evolution of those systems. Therefore the dynamics of galaxies can be described by a collisionless system, in which the stars move only under the influence of the mean gravitational potential $\Phi(\boldsymbol{r}, t)$ generated by all the other stars.

In the analytic approach (for $t_{\mathrm{r}} \rightarrow \infty$ ) the galaxy can be represented by a continuous mass distribution with a distribution function $f(\boldsymbol{r}, \boldsymbol{v}, t)$. The dynamics of such a system is governed by the collisionless Boltzmann equation:

$$
\frac{\partial f}{\partial t}+\boldsymbol{v} \cdot \nabla f-\nabla \Phi \cdot \frac{\partial f}{\partial \boldsymbol{v}}=0
$$


The gravitational field $\Phi(\boldsymbol{r}, t)$ can be obtained from the distribution function (or density distribution) by using the Poisson equation

$$
\nabla^{2} \Phi=4 \pi \mathrm{G} \int f(\boldsymbol{r}, \boldsymbol{v}, t) d^{3} \boldsymbol{v}=4 \pi \mathrm{G} \rho(\boldsymbol{r}, t)
$$

where $\rho(\boldsymbol{r})$ is the volume density and G is the gravitational constant. So in general, if the distribution function at some given time $t_{0}$ is known, we can use Eq. 3.3 to derive $f(\boldsymbol{r}, \boldsymbol{v}, t)$ at time $t$. The Boltzmann equation, however, is a first-order partial differential equation with six independent spatial variables and time, and therefore an analytic solution can be derived only for a very few specific distribution functions (see, for example, Fridman \& Polyachenko 1984).

A numerical approach to study the dynamics of galaxies are $N$-body realisations of a given distribution function. In this method, $f(\boldsymbol{r}, \boldsymbol{v}, t)$ is sampled at some given time $t_{0}$ by a set of $N$ discrete points with masses $m_{i}$ :

$$
f\left(\boldsymbol{r}, \boldsymbol{v}, t_{0}\right) \rightarrow f_{N}\left(\boldsymbol{r}_{i}, \boldsymbol{v}_{i}, t_{0}\right)=\sum_{i=1}^{N} m_{i} \delta^{3}\left(\boldsymbol{r}-\boldsymbol{r}_{i}\right) \delta^{3}\left(\boldsymbol{v}-\boldsymbol{v}_{i}\right)
$$

In this representation the sampling points are often referred to as bodies or particles and it can be shown that with this approach the phase space density always equals the mass volume density. One has to keep in mind that the particles act as tracers of the phase-space distribution and cannot be identified as individual stars. The evolution in time of $f$ can then be calculated by explicit numerical integration of the standard Newtonian equations of motion for each particle $i$, as given by

$$
\frac{d \boldsymbol{r}_{i}}{d t}=\boldsymbol{v}_{i}
$$

and

$$
\frac{d \boldsymbol{v}_{i}}{d t}=\sum_{j \neq i}^{N} \frac{\mathrm{G} m_{j}\left(\boldsymbol{r}_{j}-\boldsymbol{r}_{i}\right)}{\left|\boldsymbol{r}_{j}-\boldsymbol{r}_{i}\right|^{3}}
$$

This approach can be interpreted as a Monte-Carlo method of solving Eq. 3.3 (White 1982). Because the particle number $N$, which can be simulated with state-of-the-art super-computers, is still many orders of magnitude smaller than the actual number of stars in a galaxy, there is some need to reduce the discreteness effects introduced, e.g. by the singularity in Eq. 3.7. To reduce the effects of two-body relaxation (or graininess of the sampled distribution function) and to avoid the numerical singularities in the acceleration term at $\boldsymbol{r}_{j}=\boldsymbol{r}_{i}$, a softened form of the acceleration term (Eq. 3.7) is generally used, such as:

$$
\frac{d \boldsymbol{v}_{i}}{d t}=\sum_{j \neq i}^{N} \frac{\mathrm{G} m_{j}\left(\boldsymbol{r}_{j}-\boldsymbol{r}_{i}\right)}{\left[\left(\boldsymbol{r}_{j}-\boldsymbol{r}_{i}\right)^{2}+\epsilon^{2}\right]^{3 / 2}}
$$


where $\epsilon$ is referred to as the softening length. The softening length can be introduced in a self-consistent way, i.e. with the Poisson equation being satisfied, by smoothing out the 'density' of a particle in Eq. 3.5 as

$$
\delta^{3}\left(\boldsymbol{r}-\boldsymbol{r}_{i}\right) \rightarrow \frac{3}{4 \pi} \frac{\epsilon^{2}}{\left(\left|\boldsymbol{r}-\boldsymbol{r}_{i}\right|^{2}+\epsilon^{2}\right)^{5 / 2}}
$$

Applying a softening to the force law always affects the spatial resolution of the simulation. For the best choice of the softening in $N$-body simulations see, for example, Merrit (1996), Athanassoula et al. (2000) and Dehnen (2001).

\subsection{Stellar orbits in barred galaxies}

Disc galaxies - in contrast to elliptical galaxies and spheroidal galaxy components like a halo or bulge - are rotationally supported system, i.e. most of the stars in the disc rotate about the galactic centre on quasi-circular orbits, which are confined close to the equatorial plane of the disc. The rotation of the disc is differential, with the angular velocity of the stars varying with radius. Stellar orbits can be considered as the building blocks of galaxies and are the backbone for studying the structure and dynamics of galaxies (e.g., Athanassoula et al. 1983). In this section we give a brief summary of the main properties of stellar orbits (see, for example, Binney \& Tremaine 1987 and the review article by Sellwood \& Wilkinson 1993) and the basic methods, which are intensively used in this work for analysing the numerical simulations. In the last part of this section, we will also briefly discuss the connection between the gas-dynamics and periodic orbits.

\subsubsection{Epicyclic approximation}

Let us first consider two-dimensional planar orbits, i.e. in the equatorial plane of the disc, in an axisymmetric and time-invariant potential $\Phi(R, \theta, z=0)$, in which the angular momentum $J=R^{2} \dot{\theta}$ of a star is a conserved quantity. The general solution can be obtained by solving the equations of motion of a test particle in the potential:

$$
\ddot{R}-R \dot{\theta}=-\frac{\partial \Phi}{\partial R} \quad \text { and } \quad \dot{J}=-\frac{\partial \Phi}{\partial \theta} .
$$

The simplest solution in this case is a circular orbit with a home radius $R_{\mathrm{c}}$ and a circular frequency

$$
\Omega_{\mathrm{c}}(R)=\sqrt{\frac{1}{R} \frac{\partial \Phi}{\partial R}} .
$$

In Fig. 3.1 (panel a) we show an example of a circular orbit in an axisymmetric potential. The circular orbits are among other things characterized by the fact that 


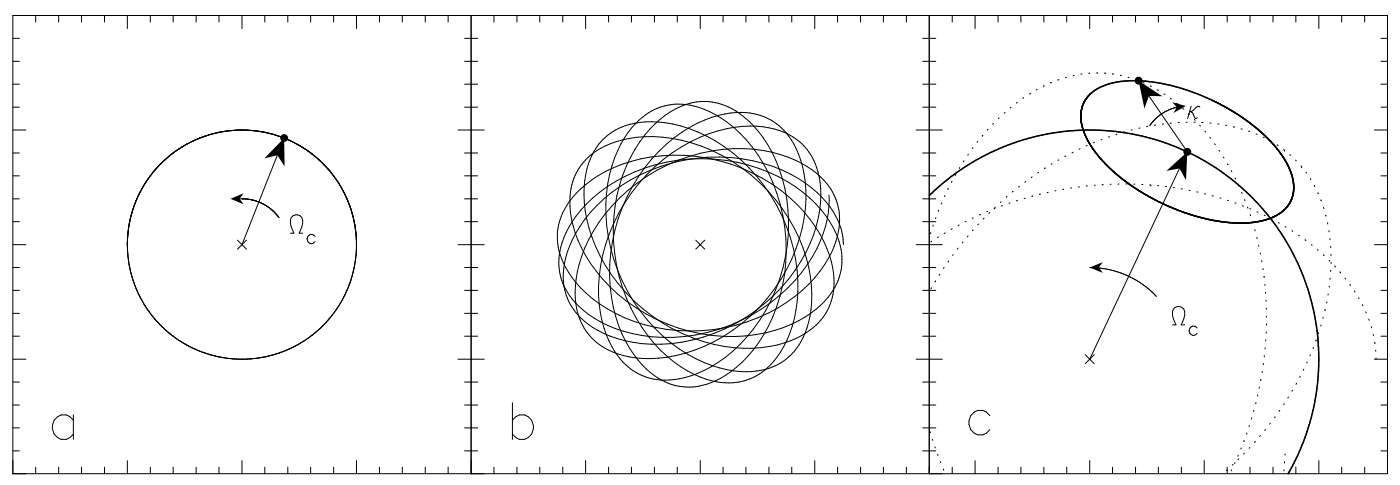

Figure 3.1: Epicyclic approximation. In panel (a) we show a circular orbit with frequency $\Omega_{\mathrm{c}}$. Panel (b) shows a nearly circular orbit in the same potential. Panel (c) shows the epicyclic approximation of the same orbit, in which the motion is described by the guiding centre and an epicycle.

they are the orbits with minimum energy for a given $J$. Stars with higher energies (and the same $J$ ) describe rosette-like orbits instead, which are in general not closed (Fig 3.1 b) in the inertial frame of reference. For orbits which are not far from being circular, the motion of the stars can be approximated by a harmonic epicycle about a guiding centre, which orbits at $\Omega_{\mathrm{c}}$ (Lindblad 1927), as illustrated in Fig. $3.1 \mathrm{c}$. This can be shown by writing the equations of motion in terms of displacements from a circular motion (see also next section).

The radial Lindblad epicyclic frequency of a star is then given by

$$
\kappa(R)=\left[4 \Omega_{\mathrm{c}}^{2}+R \frac{d \Omega_{\mathrm{c}}^{2}}{d R}\right]^{1 / 2} .
$$

In this linear approximation the two frequencies $\Omega_{\mathrm{c}}$ and $\kappa$ describing the star's motion, can be considered as the star's natural frequencies in the galactic potential. The quasi-circular orbits are only closed in an inertial frame of reference, if and only if $\Omega_{\mathrm{c}}=\frac{l}{m} \kappa$, with integers $l$ and $m .{ }^{1}$ The orbit closes after $|l|$ revolutions about the galactic centre and $m$ radial oscillations.

\subsubsection{Orbits in barred potentials}

In this section we describe stellar orbits in a barred potential. We assume that the bar rotates steadily with a pattern speed $\Omega_{\mathrm{p}}$. The equations of motion in a frame of reference rotating with a constant pattern speed $\Omega_{\mathrm{p}}$ are then given by:

$$
\ddot{\boldsymbol{r}}=-\boldsymbol{\nabla} \Phi-2\left(\boldsymbol{\Omega}_{\mathrm{p}} \times \dot{\boldsymbol{r}}\right)-\boldsymbol{\Omega}_{\mathrm{p}} \times\left(\boldsymbol{\Omega}_{\mathrm{p}} \times \boldsymbol{r}\right),
$$

\footnotetext{
${ }^{1}$ It is always possible to choose a frame of reference, rotating with a constant frequency $\Omega_{\text {frame }}$ in which such an orbit appears to be closed, i.e. satisfying the condition $\Omega_{\mathrm{c}}-\Omega_{\text {frame }}=\frac{l}{m} \kappa$.
} 
where the second and third term on the right-hand side of Eq. 3.13 represent the coriolis and the centrifugal forces, respectively. Using cylindrical coordinates, choosing $\Omega_{\mathrm{p}}=\Omega_{\mathrm{p}} \boldsymbol{e}_{z}$ and setting $z=\dot{z}=\ddot{z}=0$, the equations of motion become componentwise, in $R$ and $\varphi$, respectively:

$$
\begin{aligned}
& \left(\ddot{R}-R \dot{\varphi}^{2}\right)=-\frac{\partial \Phi}{\partial R}+2 R \dot{\varphi} \Omega_{\mathrm{p}}+R \Omega_{\mathrm{p}}^{2} \\
& (2 \dot{R} \dot{\varphi}+R \ddot{\varphi})=-\frac{1}{R} \frac{\partial \Phi}{\partial \varphi}-2 \dot{R} \Omega_{\mathrm{p}}
\end{aligned}
$$

Furthermore we use the first-order perturbation ansatz and write the gravitational potential as:

$$
\Phi(R, \varphi)=\Phi_{0}(R)+\Phi_{1}(R, \varphi),
$$

with $\left|\Phi_{1} / \Phi_{0}\right| \ll 1$. We further use $R(t)=R_{0}+R_{1}(t)$ and $\varphi(t)=\varphi_{0}(t)+\varphi_{1}(t)$. The zeroth-order terms in Eq. 3.14 then yields:

$$
R_{0} \dot{\varphi}_{0}^{2}=\left(\frac{d \Phi_{0}}{d R}\right)_{R_{0}}-2 R_{0} \dot{\varphi}_{0} \Omega_{\mathrm{p}}-R_{0} \Omega_{\mathrm{p}}^{2} \quad
$$

which is equivalent to the equation for centrifugal equilibrium at radius $R_{0}$ :

$$
R_{0}\left(\dot{\varphi}_{0}+\Omega_{\mathrm{p}}\right)^{2}=\left(\frac{d \Phi_{0}}{d R}\right)_{R_{0}}
$$

If we define $\Omega_{0} \equiv \Omega_{\mathrm{c}}\left(R_{0}\right)$, where $\Omega_{\mathrm{c}}$ is the circular frequency as given by Eq. $3.11 \mathrm{in}$ the axisymmetric potential $\Phi_{0}$, we get the angular frequency of the guiding centre $\left(R_{0}, \varphi_{0}\right)$ from Eq. 3.18 as:

$$
\dot{\varphi}_{0}=\Omega_{0}-\Omega_{\mathrm{p}} .
$$

We assume here that $\Omega_{\mathrm{p}}>0$, so that for prograde (or direct) and retrograde orbit are $\Omega_{0}>0$ and $\Omega_{0}<0$, respectively. We further choose $t=0$ such that

$$
\varphi_{0}(t)=\left(\Omega_{0}-\Omega_{\mathrm{p}}\right) t
$$

For the first-order terms in the linearized equation of motion Eq. 3.14 we get:

$$
\begin{aligned}
\ddot{R}_{1} & -2 R_{0} \dot{\varphi}_{0} \dot{\varphi}_{1}-R_{1} \dot{\varphi}_{0}^{2}= \\
& -\left(\frac{d \Phi_{1}}{d R}\right)_{R_{0}}-R_{1}\left(\frac{d^{2} \Phi_{0}}{d R^{2}}\right)_{R_{0}}+2 R_{0} \dot{\varphi}_{1} \Omega_{\mathrm{p}}+2 R_{1} \dot{\varphi}_{0} \Omega_{\mathrm{p}}+R_{1} \Omega_{\mathrm{p}}^{2}
\end{aligned}
$$


By rearranging the terms and using Eq. 3.19, we get:

$$
\ddot{R}_{1}+\left[\left(\frac{d^{2} \Phi_{0}}{d R^{2}}\right)-\Omega^{2}\right]_{R_{0}} R_{1}-2 R_{0} \dot{\varphi}_{1} \Omega_{0}=-\left(\frac{d \Phi_{1}}{d R}\right)_{R_{0}},
$$

where $\left[\left(d^{2} \Phi_{0} / d R^{2}\right)-\Omega^{2}\right]_{R_{0}}$ is the epicyclic frequency $\kappa_{0}=\kappa\left(R_{0}\right)$ as introduced in Eq. 3.12. In a similar way we get for the first-order terms in Eq. 3.15:

$$
2 \dot{R}_{1} \dot{\varphi}_{0}+R_{0} \ddot{\varphi}_{1}=-\left(\frac{1}{R} \frac{\partial \Phi_{1}}{\partial \varphi}\right)_{R_{0}}-2 \dot{R}_{1} \Omega_{\mathrm{p}}
$$

which yields

$$
\ddot{\varphi}_{1}+2 \Omega_{0} \frac{\dot{R}_{1}}{R_{0}}=-\frac{1}{R_{0}^{2}}\left(\frac{\partial \Phi_{1}}{\partial \varphi}\right)_{R_{0}} .
$$

The results obtained so far did not make any assumptions about the form of the perturbing potential. For further analysis we now choose a specific form of $\Phi_{1}$ and set

$$
\Phi_{1}(R, \varphi)=\Phi_{\mathrm{b}}(R) \cos (m \varphi)
$$

Since bars show roughly a two-fold symmetry, we expect $m=2$ to be the dominant case for barred galaxies. We further assume that $\varphi_{1} \ll \varphi_{0}$, i.e., $\varphi(t) \approx \varphi_{0}(t)=$ $\left(\Omega_{0}-\Omega_{\mathrm{p}}\right) t$. The equations of motion can now be written as:

$$
\begin{aligned}
& \ddot{R}_{1}+\kappa_{0}^{2} R_{1}-2 R_{0} \Omega_{0} \dot{\varphi}_{1}=-\left(\frac{\partial \Phi_{\mathrm{b}}}{\partial R}\right)_{R_{0}} \cos \left[m\left(\Omega_{0}-\Omega_{\mathrm{p}}\right) t\right] \\
& \ddot{\varphi}_{1}+2 \Omega_{0} \frac{\dot{R}_{1}}{R_{0}}=\frac{m \Phi_{b}\left(R_{0}\right)}{R_{0}^{2}} \sin \left[m\left(\Omega_{0}-\Omega_{\mathrm{p}}\right) t\right] .
\end{aligned}
$$

Integrating Eq. 3.27, we obtain:

$$
\dot{\varphi}_{1}=-2 \Omega_{0} \frac{R_{1}}{R_{0}}-\frac{\Phi_{\mathrm{b}}\left(R_{0}\right)}{R_{0}^{2}\left(\Omega_{0}-\Omega_{\mathrm{p}}\right)} \cos \left[m\left(\Omega_{0}-\Omega_{\mathrm{p}}\right) t\right]+\text { const. },
$$

and substituting this expression in Eq. 3.26:

$$
\ddot{R}_{1}+\kappa_{0}^{2} R_{1}=-\left[\frac{d \Phi_{\mathrm{b}}}{d R}+\frac{2 \Omega_{\mathrm{c}} \Phi_{\mathrm{b}}}{R\left(\Omega_{\mathrm{c}}-\Omega_{\mathrm{p}}\right)}\right]_{R_{0}} \cos \left[m\left(\Omega_{0}-\Omega_{\mathrm{p}}\right) t\right]+\text { const. }
$$

This equation corresponds to the equation of motion for a harmonic oscillator of natural frequency $\kappa_{0}$ that is driven at frequency $m\left(\Omega_{0}-\Omega_{\mathrm{p}}\right)$. The general solution of Eq. 3.29 is thus given by:

$$
R_{1}(t)=C_{1} \cos \left(\kappa_{0} t+\psi\right)-\left[\frac{d \Phi_{\mathrm{b}}}{d R}+\frac{2 \Omega_{\mathrm{c}} \Phi_{\mathrm{b}}}{R\left(\Omega_{\mathrm{c}}-\Omega_{\mathrm{b}}\right)}\right]_{R_{0}} \frac{\cos \left[m\left(\Omega_{0}-\Omega_{\mathrm{p}}\right) t\right]}{\Delta}
$$


where $C_{1}$ and $\psi$ are arbitrary constants, and $\Delta$ is defined as:

$$
\Delta=\kappa_{0}^{2}-m^{2}\left(\Omega_{0}-\Omega_{\mathrm{p}}\right)^{2}
$$

We can use Eq. 3.20 to eliminate $t$ and obtain:

$$
R_{1}\left(\varphi_{0}\right)=C_{1} \cos \left(\frac{\kappa_{0} \varphi_{0}}{\Omega_{0}-\Omega_{p}}+\psi\right)+C_{2} \cos \left(m \varphi_{0}\right)
$$

where

$$
C_{2}=-\frac{1}{\Delta}\left[\frac{d \Phi_{\mathrm{b}}}{d R}+\frac{2 \Omega_{\mathrm{c}} \Phi_{\mathrm{b}}}{R\left(\Omega_{\mathrm{c}}-\Omega_{\mathrm{p}}\right)}\right]_{R_{0}} .
$$

Thus the full solution of the equations of motion is a combination of the distorted path of the guiding centre and epicycles. With $C_{1} \neq 0$ the solution describes nonclosed loop orbits, while for $C_{1}=0$ the solution is periodic in $\varphi_{0}$ with a period of $2 \pi / m$. The periodic orbits play an important role in understanding the dynamics of barred galaxies and we will come back to them later on in this section.

\section{Dynamical resonances in the linear regime}

When one of the terms on the right-hand side of Eq. 3.32 become singular, the orbit is in resonance with the perturbation. The resonance condition from the linear theory is thus given by:

$$
\Omega_{\mathrm{p}}=\Omega_{\mathrm{c}}(R) \pm \frac{l}{m} \kappa(R)
$$

The main planar resonances, which play a central role in the study of bars and spirals, are the

1. corotation resonance (hereafter $\mathrm{CR}$ )

$$
\Omega_{\mathrm{p}}=\Omega_{\mathrm{c}} \quad(l=0)
$$

2. outer Lindblad resonance (hereafter OLR)

$$
\Omega_{\mathrm{p}}=\Omega_{\mathrm{c}}+\frac{\kappa}{2} \quad(l=+1, m=2)
$$

3. inner Lindblad resonance (hereafter ILR)

$$
\Omega_{\mathrm{p}}=\Omega_{\mathrm{c}}-\frac{\kappa}{2} \quad(l=-1, m=2)
$$

4. ultra-harmonic resonance (hereafter UHR)

$$
\Omega_{\mathrm{p}}=\Omega_{\mathrm{c}}-\frac{\kappa}{4} \quad(l=-1, m=4)
$$




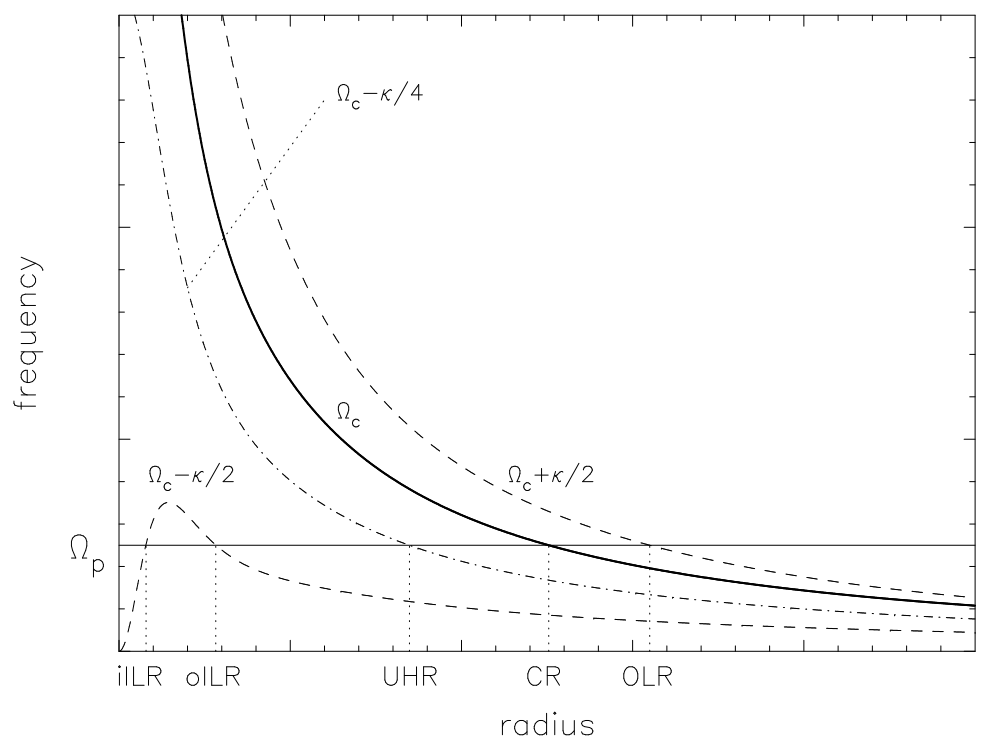

Figure 3.2: Linear resonance diagram. The curves show the circular frequency $\Omega_{\mathrm{c}}$ (full line), $\Omega_{\mathrm{c}} \pm \kappa / 2$ (dashed lines) and $\Omega_{\mathrm{c}}-\kappa / 4$ (dotted-dashed line). The horizontal and vertical lines indicate the pattern speed $\Omega_{\mathrm{p}}$ of the bar and the position of the corresponding resonances, respectively.

Please note that the resonance condition for the ILR is equivalent to the condition for an orbit in a rotating frame of reference to be closed. Thus orbits which appear closed in the frame of reference co-rotating with the bar can always be considered as resonance orbits. This result leads to some ambiguity in the definition of the Lindblad resonances (see Athanassoula 2003 for a discussion).

If the potential of a galaxy is known, we can use Eq. 3.34 to determine the position of the resonances in the disc. In Fig. 3.2 we show a resonance diagram, which has been obtained by plotting the frequencies $\Omega_{\mathrm{c}}$ and $\Omega_{\mathrm{c}} \pm(l / \mathrm{m}) \kappa$ as a function of radius (for $l=1$ and $m=2,4$ ). At those radii where the line $\Omega_{\mathrm{p}}=$ const. intersects with the frequency curves we locate a resonance. As can be easily seen from this plot, the number of inner Lindblad resonances can vary, depending on the value of $\Omega_{\mathrm{p}}$. In the case shown we have two ILRs, which are appropriate called inner and outer ILR (hereafter iILR and oILR, respectively). For higher values of $\Omega_{\mathrm{p}}$ there may exist only one or even no ILR, which is the case when $\Omega_{\mathrm{p}}$ equals or exceeds the maximum of the $\Omega_{\mathrm{p}}=\Omega_{\mathrm{c}}-\kappa / 2$ curve, respectively.

As can be seen from Eq. 3.34, the orientation of the orbits changes at each principle resonance (for fixed $m$ ), i.e. the orbits are aligned with the bar for $C_{2}<0$ and perpendicular to the bar for $C_{2}>0$. The latter is the case in the region between the inner and the outer ILR. We will later identify those orbits trapped between the iILR and the oILR as the $x_{1}$ and $x_{2}$ orbits in the non-linear regime. Indeed the 
presence of the $x_{2}$ orbits is a necessary condition for the presence of an ILR (see Athanassoula 2003).

The linear approximation described above is only valid for weak perturbations. For the case of a strong bar one has to switch to an orbit analysis in the non-linear regime, which will be discussed in the following sections. The above analysis helps us, however, to understand the properties of stellar orbits in barred potentials.

\subsubsection{Periodic orbits}

In this section we describe periodic orbits in barred potentials, i.e. orbits which make a closed figure in a frame of reference rotating with the bar. These orbits provide useful information to study the stellar dynamics of barred galaxies (see, for example, Contopoulos \& Mertzanides 1977; Contopoulos \& Papayannopoulos 1980; Contopoulos \& Grosbøl 1989; Athanassoula 1992b; etc.) and can be considered as the backbone of galactic structure (Athanassoula et al. 1983). Although the stars in a galaxy will not be on periodic orbits, the latter provide useful information, because they trap non-closed orbits around them. For simplicity we confine ourselves again to two-dimensional planar orbits only, which are confined to the equatorial plane of the disc. A description of the three-dimensional structure of orbits in barred galaxies is given in, e.g., Pfenniger (1984), Pfenniger \& Friedli (1991), Berentzen et al. (1998) and the series of papers by Skokos, Patsis \& Athanassoula (2002a, 2002b) and Patsis, Skokos \& Athanassoula (2002, 2003).

In this section we describe the lowest order periodic orbits. The most important orbits are the ones which are bi-symmetric $m=2$ with respect to the bar and close after one orbit around the centre in the rotating frame of reference. In the frame of reference corotating with the bar neither the particles energy nor its angular momentum is a conserved quantity. However, there exists another integral of motion ${ }^{2}$. In the rotating frame of reference the only known integral of motion is the Jacobi integral:

$$
E_{\mathrm{J}}=\frac{1}{2}|\boldsymbol{v}|^{2}+\Phi_{\mathrm{eff}}(\boldsymbol{r})
$$

where $\Phi_{\text {eff }}=\Phi-\frac{1}{2} \Omega_{\mathrm{p}}^{2} R^{2}$ is the effective potential. Because $E_{\mathrm{J}}$ has the units of an energy, it is often referred to as the Jacobi energy and can be thought of as an effective energy of a star.

\section{Characteristic diagrams}

In the characteristic diagram the periodic orbits are plotted with respect to their Jacobi integral $E_{\mathrm{J}}$ and the $y$-intercept value with the $x=0$ plane. In such a diagram

\footnotetext{
${ }^{2} \mathrm{An}$ integral of motion is a time-independent function of the coordinates, which is constant along a trajectory in phase space.
} 

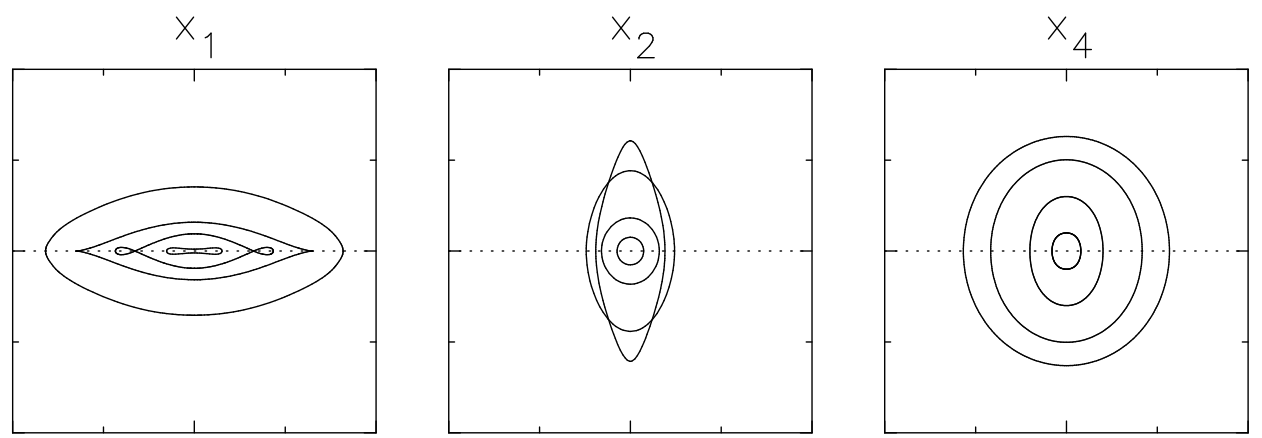

Figure 3.3: Main periodic orbit families. The panels from left to right show examples of the $x_{1}, x_{2}$ and $x_{4}$ orbits in a barred potential. The orientation of the bar's major axis is indicated by the horizontal dotted line.

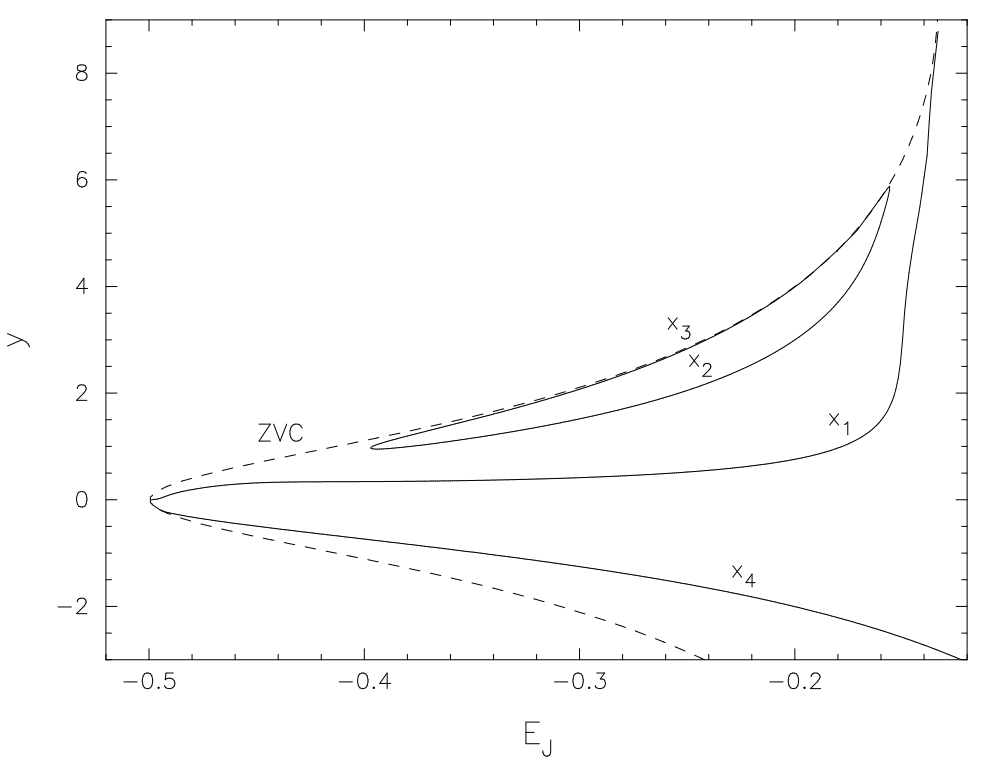

Figure 3.4: Characteristic diagram of the main orbit families. We show the characteristic curves (full lines) of the main planar periodic orbits within corotation in a barred potential. The dashed line shows the zero-velocity curve (ZVC).

the periodic orbits are not scattered, but are grouped in families in the form of curves, known as characteristic curves in this diagram. In Fig. 3.3 we show some examples of different periodic orbits and in Fig. 3.4 the corresponding characteristic diagram of the main orbit families in a barred potential (within the corotation), using the notation of Contopoulos \& Papayannopoulos (1980). The dashed curve is the zero-velocity curve (hereafter ZVC), which delineates the accessible region in the plane based on energy considerations. 


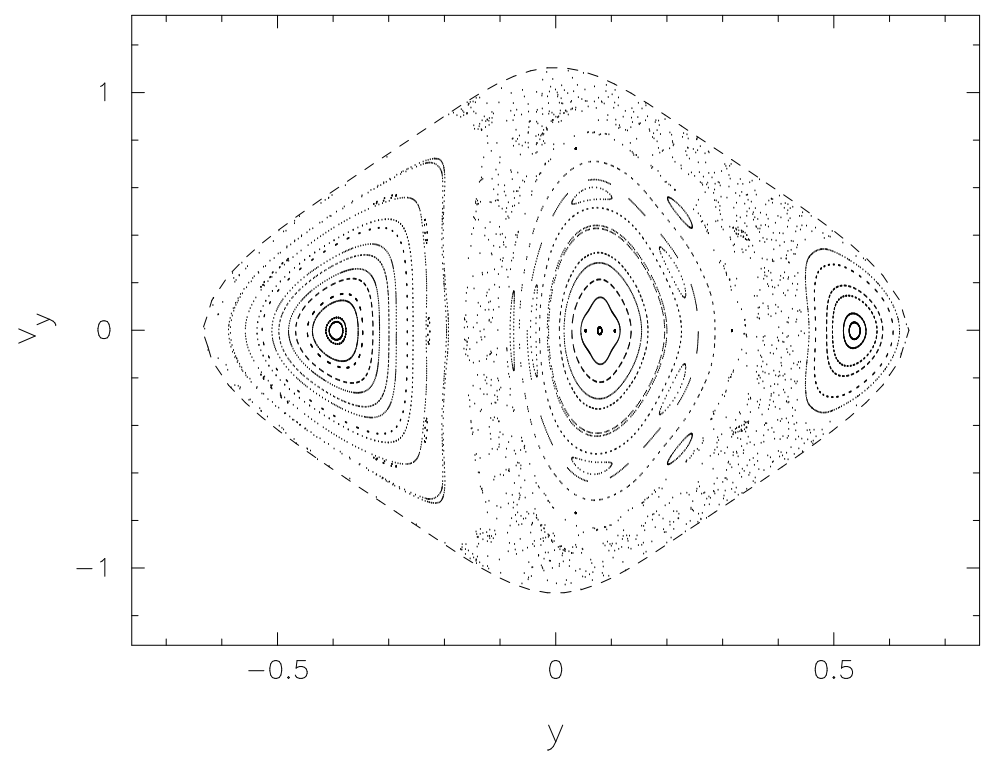

Figure 3.5: Example of a surface of section. The three fix-points at $v_{y}=0$ are, from left to right, the periodic orbits of the $x_{4}$ (retrograde), the $x_{1}$ and the $x_{2}$ families (both direct or prograde). Trapped around them are the multi- and non-periodic orbits, forming the invariant curves and islands. A ergodic region is also present in this case, separating the $x_{1}$ and $x_{2}$ region.

The $x_{1}$ orbit family consists of orbits, which predominately give the bar its structure. They are elongated along the bar major axis and are direct orbit, i.e. the star's motion is in the same sense as the bar. In the rotating reference frame of the bar they move in and out, twice for once around the centre (2/1). The $x_{4}$ orbits are retrograde $2 / 1$ orbits, slightly elongated perpendicular to the bar. The $x_{2}$ orbits, like $x_{1}$, are also direct $2 / 1$ orbits, but elongated perpendicular to the bar. As mentioned before, their presence is indicative of an ILR (or more than one) in the non-linear regime.

\section{Surfaces of section}

For a given dynamical system the orbital properties can be obtained from the surfaces of section (hereafter SOS), originally introduced by Poincaré (1892). To construct an SOS, we plot for a given $E_{\mathrm{J}}$ the intersections of orbits with the plane $x=0$ for trajectories which have $\dot{x}>0$. Thus we have for each orbit a succession of points $P_{i}=(y, \dot{y})$ called consequents. In such a diagram periodic orbits are represented by fixed points, i.e. an $m$-periodic orbit is represented by $m$ fixed points. In a galactic-like potential, however, most orbits are not periodic. Nevertheless, the periodic orbits play an important role, because they trap regions of phase space about 
them. These trapped regions are called regular regions and the orbits, which reside in them are called regular orbits. Their motion is confined to a $2 \mathrm{D}$ surface, referred to as an invariant torus and which surrounds the parent periodic orbit. Orbits, which are not trapped, are called irregular orbits. Unlike the regular orbits, they are not restricted to a subsurface and may wander throughout the non-regular regions of the phase space, at least within energy considerations. An example of a SOS for a barred potential can be seen in Fig. 3.5.

The regular orbits in this diagram form the closed curves, which surround the fixed points of the parent periodic orbits at the centres. These curves are referred to as invariant curves and are a cross-section of the invariant tori. The left side of the diagram, with $y<0$, represents the retrograde orbits, while the right side, with $y>0$, represents the direct or prograde orbits. In Fig. 3.5 there are three regular regions, which from left to right are associated with the $x_{4}, x_{1}$ and the $x_{2}$ orbital families, respectively. Because the presence of the $x_{2}$ orbits is indicative of the inner Lindblad resonances, the SOS provide a tool to find the resonances in the non-linear regime and to estimate the importance of each orbit family by the phase space region it occupies.

\subsubsection{Gas-dynamics in barred galaxies}

In the introduction we already described the importance of gas on the dynamics of barred galaxies and some of the different features it may produce. In this section we will briefly describe the response of gas to a barred potential in terms of the periodic orbits.

A relation between the gas flow and the main periodic orbits in barred galaxies has already been pointed out by Prendergast (1983) and and Sanders et al. (1983). The velocity dispersion in the interstellar medium is in general an order of magnitude lower than the streaming motion of the gas and the influence of pressure will thus be negligible. Therefore the gas streamlines must under this condition coincide with the periodic orbits in the potential. In contrast to the stellar orbits, however, the gas streamlines in a laminar flow cannot cross, since the gas must have a unique streaming velocity at each point in the flow. Thus if the periodic orbits are not properly nested and somehow cross each other (e.g., by superposition of $x_{1}$ and $x_{2}$ orbits or loop $x_{1}$ orbits; compare Fig. 3.3), pressure or viscous forces will act such as to prevent crossing streamlines. If the main families of periodic orbits intersect shocks are formed (Prendergast 1983; Sanders et al. 1983). Through changes in energy or angular momentum the gas may change its orbit, resulting in a radial redistribution of the gas, which within co-rotation is generally directed inwards. Athanassoula (1992b, 1994) found how the location and the shape of the shock loci, as well as the strength and the inflow rates, depend on the dynamical parameter of the galaxy. 


\section{Numerical methods}

\subsection{The algorithms}

As has been shown in the previous section, the dynamics of a galaxy can be described by that of a collisionless fluid, with the stars moving in the mean gravitational potential generated by all the other stars. Thus the main task in numerical simulations is the computation of the forces exerted by the stars. To simulate a galaxy with its real numer of stars (i.e., with $N \approx 10^{11}$ ) is not possible even with state-of-the-art supercomputers. But even with the particle numbers feasible today, i.e. some $10^{6}, N$-body simulations remain a vastly time-consuming task.

The different $N$-body methods can be categorised basically in the following groups (e.g., see review by Sellwood 1987; Hockney \& Eastwood 1988):

1. direct methods (or particle-particle methods):

In direct methods the gravitational interaction between the particles is calculated by taking the direct sum over all other particles. Since this step is done for all particles the computational time is roughly proportional to $N^{2}$, while the integration of the equations of motion only goes with $N$. Direct methods are by far the slowest ones, albeit the most accurate. Furthermore they have the advantage, that they do not set any constraints on the symmetry of the simulated system.

2. hierarchical methods (or tree methods):

The basic concept of the tree methods is to approximate the forces of a group of distant particles by some sort of truncated expansion instead of individual pair-wise sums . The particles are typically binned in a hierarchical tree structure. The forces accummulated for any given particle in a descent through the tree. A popular implementation is the hierarchical tree method by Barnes \& Hut (1986). The computational cost of this method scales with $O(N \log N)$. Tree methods are, as direct methods, gridless and have no preferred geometry.

\section{3. field expansion methods:}

The gravitational potential or density distribution is approximated by a truncated expansion in some set of basis function, such as spherical harmonics. The basis set has to be chosen depending on the geometry of system under study. While very fast and produce no two-body relaxation they are best suited to studying equilibrium distributions. 


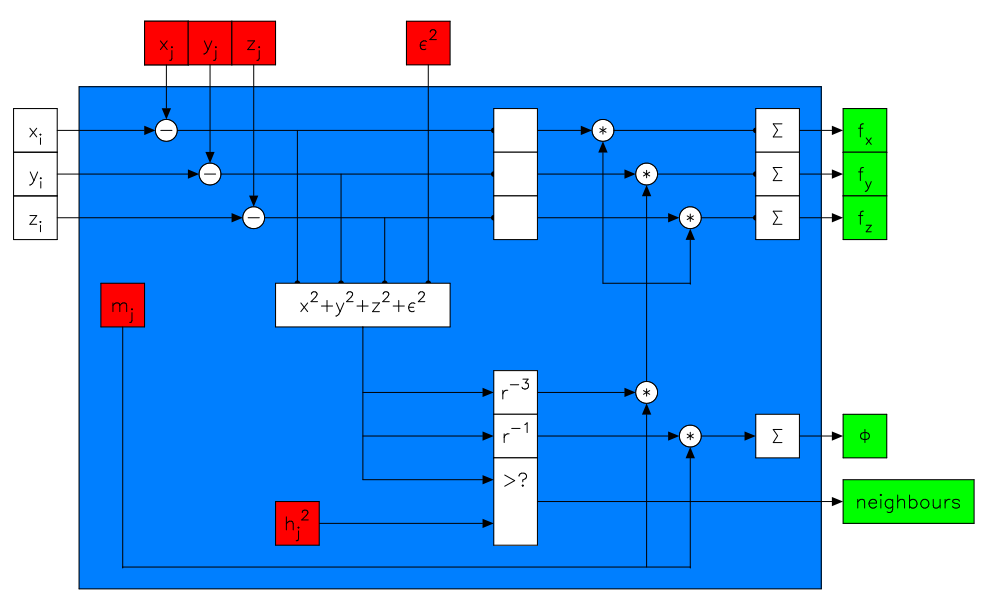

Figure 4.1: Schematic illustration of the GRAPE-3 chip. The red and green boxes indicate model input and output values, respectively, for a given set of coordinates $\left(x_{i}, y_{i}, z_{i}\right)$.

4. grid methods (or particle-mesh):

In this method the mass of a particle is distributed over several grid points and the forces on the particles are then interpolated after calculating the gravitational potential and the force at the grid points. Their advantage is in their speed which comes from the use of the FFT to compute the forces from the density. The main disadvantage of grid methods is, however, that it sets contraints on the geometry of the system and thus sets limits on the spatial resolution of the simulation.

\subsubsection{The special-purpose hardware GRAPE}

The direct particle-particle method is the most straight-forward one among the $N$ body methods. But the direct integration approach has a high computational cost: $O\left(N^{2}\right)$ operations are required to evaluate the forces on all $N$ particles. In order to simulate tens of thousands of particles by a highly-accurate particle-particle method, one can expect the simulations to perform at least $10^{14}$ floating operations. The special-purpose hardware GRAPE (short for GRAvity PipE) was designed and built by a group of astro-physicists at the University of Tokyo (see Sugimoto et al. 1990). The GRAPE-board is a piece of hardware attached to a host workstation, and accelerates the Newtonian force calculation. The basic concept of GRAPE is that the host computer, usually a general-purpose workstation running UNIX, sends the positions and masses of particles to the GRAPE hardware. Then the GRAPE hardware calculates the interaction between particles, i.e. the gravitational potential and forces. The design of a GRAPE chip is shown in Fig. 4.1. The GRAPE-3 hardware 
was originally released in 1991 and reaches a peak velocity of 15 Gflops (flops $=$ floating point operations per second).

With this type of hardware it is possible to evolve a system of a few $100 \mathrm{~K}$ particles and a few million particles with the follow-up version GRAPE-5 (Kawai et al. 2000). A description of the GRAPE-3 system at the Sternwarte Göttingen, which among others has been used for the simulations presented in this work, is given in Appendix C.

\subsubsection{The $N$-body/SPH code}

In this section we describe the basic concepts of the numerical methods used for the simulations presented in this work. The $N$-body/SPH code used in the simulations (called FTM; short for Fast Tree Method) was developed by C.H. Heller (see Heller 1991) to study encounters between protostellar discs. The version used in this work has been updated, including support for the GRAPE hardware.

\section{Gravity}

For systems with a large number of stars, one can adopt a statistical description as described in Sec 3.1, instead of tracking individual stars. The evolution of the distribution function $f(\mathbf{r}, \mathbf{v}, \mathbf{t})$ is governed by the collisionless Boltzmann equation (Eq. 3.3). where the gravitational potential $\phi(\boldsymbol{r}, t)$ is given by Poisson's equation (Eq. 3.4). The gravitational forces between gas, stars and halo particles are computed by a direct summation method supporting the GRAPE-3Af hardware. The Keplerian potential between two particles is replaced by the softened form in order to prevent numerical divergencies and to reduce the two-body relaxation. The softened potential gives a force

$$
\boldsymbol{F}_{j}=\sum_{i \neq j}^{N} \frac{\mathrm{G} m_{i} m_{j} \cdot\left(\boldsymbol{r}_{i}-\boldsymbol{r}_{j}\right)}{\left(\left|\boldsymbol{r}_{i}-\boldsymbol{r}_{j}\right|^{2}+\epsilon^{2}\right)^{3 / 2}}
$$

In the simulations presented in this work the direct force calculation has been performed using the GRAPE-hardware.

\subsubsection{Smoothed particle hydrodynamics}

Smoothed particle hydrodynamics (hereafter SPH) has first been introduced in an astrophysical context by Lucy (1977) and Gingold \& Monaghan (1977). SPH is a fully Lagrangian method, in which the fluid elements constituting the system are sampled and represented by a set of points, which are referred to as particles. The advantage of this method is, that it is not based on grids, which would constrain the dynamical range in spatial resolution and the global geometry of the system being studied. The dynamical equations are obtained from the Lagrangian form of the 
hydrodynamic conservation laws. In this section we describe the basic concepts of SPH and the general features of the applied algorithm, following the description of Monaghan (1992).

\section{Basic concept}

The mean value of any physical field, $\mathrm{A}(\boldsymbol{r})$, within a given interval can be determined by the integral interpolant

$$
\langle A(\boldsymbol{r})\rangle_{\mathrm{I}}=\int A\left(\boldsymbol{r}^{\prime}\right) W\left(\boldsymbol{r}-\boldsymbol{r}^{\prime} ; h\right) d \boldsymbol{r}^{\prime}
$$

where $W$ is an normalised interpolating kernel, $h$ the smoothing length, and the integration is done over the entire space. The interpolating kernel has the following properties:

$$
\int W\left(\boldsymbol{r}-\boldsymbol{r}^{\prime}, h\right) d \boldsymbol{r}^{\prime}=1
$$

and

$$
\lim _{h \rightarrow 0} W\left(\boldsymbol{r}-\boldsymbol{r}^{\prime}, h\right)=\delta\left(\boldsymbol{r}-\boldsymbol{r}^{\prime}\right)
$$

For practical use $W$ is chosen to be greater than zero everywhere and should be at least differentiable once. For numerical work the values of $A(\boldsymbol{r})$ are known only at a finite number of discrete points, distributed with a number density $\sum_{i=1}^{N} \delta\left(\boldsymbol{r}-\boldsymbol{r}^{\prime}\right)$. The integral interpolant in Eq. 4.2 can then expressed as

$$
\langle A(\boldsymbol{r})\rangle_{\mathrm{S}}=\sum_{i=1}^{N} \frac{f\left(\boldsymbol{r}_{i}\right)}{\left\langle n\left(\boldsymbol{r}_{i}\right)\right\rangle} W\left(\boldsymbol{r}-\boldsymbol{r}_{i}, h\right)
$$

In the following we will skip the suffix $S$ when related to finite sampling. With a mass $m_{i}$ associated with each fluid element, the density may be estimated by

$$
\langle\rho(\boldsymbol{r})\rangle=\sum_{i=1}^{N} m_{i} W\left(\boldsymbol{r}-\boldsymbol{r}_{i}, h\right) .
$$

The gradient of $A(\boldsymbol{r})$ is easily obtained from Eq. 4.2 as:

$$
\langle\nabla A(\boldsymbol{r})\rangle_{\mathrm{I}}=\int A\left(\boldsymbol{r}^{\prime}\right) \nabla W\left(\boldsymbol{r}-\boldsymbol{r}^{\prime}, h\right) d \boldsymbol{r}^{\prime}
$$

and similarly the divergence is given by:

$$
\langle\nabla \cdot A(\boldsymbol{r})\rangle_{\mathrm{I}}=\int A\left(\boldsymbol{r}^{\prime}\right) \cdot \nabla W\left(\boldsymbol{r}-\boldsymbol{r}^{\prime}, h\right) d \boldsymbol{r}^{\prime}
$$




\section{The interpolation kernel}

The three-dimensional kernel $W$ used in the code is based on a spherically symmetric spline function (Monaghan \& Lattanzio 1985) of the form

$$
W(r, h)=\frac{1}{\pi h^{3}} \begin{cases}1-\frac{3}{2} q^{2}+\frac{3}{4} q^{3} & \text { if } 0 \leq \frac{r}{h} \leq 1 \\ \frac{1}{4}(2-q)^{3} & \text { if } 1 \leq \frac{r}{h} \leq 2 \\ 0 & \text { if otherwise }\end{cases}
$$

where $r \equiv\left|\boldsymbol{r}-\boldsymbol{r}^{\prime}\right|$. This kernel has several advantages. First, it has compact support, i.e. for $r>2 h$ interactions are exactly zero. Second, the second derivative is continuous, which means, that the kernel is not sensitive to disorder and the errors in approximating the integral interpolants by summation interpolants are small provided the particle disorder is not too large. And finally, the error term in the integral interpolant is $\mathcal{O}\left(h^{2}\right)$. For the kernel given in Eq. 4.9 only particles within a radius of $2 h$ contribute to the interpolation integral.

\section{The smoothing length}

The smoothing length $h$ defines the spatial resolution as a length scale over which is averaged during the estimation process. In the FTM code we apply a spatially varying smoothing length and therefore the kernel gradient for a variable $h$ is given as:

$$
\boldsymbol{\nabla} W(r, h)=\frac{\partial W}{\partial r} \boldsymbol{\nabla} r+\frac{\partial W}{\partial h} \nabla h
$$

In general the second term is small compared to the first and can safely be ignored (Evrard 1988). In order to guarantee the total momentum conservation the equations of motion must be symmetrised in $h_{i}$ and $h_{j}$, where $i$ and $j$ denote the particles. In the FTM code we symmetrise the smoothing procedure following Hernquist \& Katz (1989) by adopting a hybrid kernel of the form:

$$
W^{\prime}\left(\left|\boldsymbol{r}-\boldsymbol{r}^{\prime}\right|, h(\boldsymbol{r}), h\left(\boldsymbol{r}^{\prime}\right)\right)=\frac{1}{2}\left[W\left(\left|\boldsymbol{r}-\boldsymbol{r}^{\prime}\right|, h\left(\boldsymbol{r}^{\prime}\right)\right)+W\left(\left|\boldsymbol{r}-\boldsymbol{r}^{\prime}\right|, h(\boldsymbol{r})\right)\right],
$$

which is a linear combination of the scatter and gather formalisms (see Hernquist $\&$ Katz 1989). The expectation values are then computed according to

$$
\langle A(\boldsymbol{r})\rangle=\int A\left(\boldsymbol{r}^{\prime}\right) \frac{1}{2}\left[W\left(\left|\boldsymbol{r}-\boldsymbol{r}^{\prime}\right|, h(\boldsymbol{r})\right)+W\left(\left|\boldsymbol{r}-\boldsymbol{r}^{\prime}\right|, h\left(\boldsymbol{r}^{\prime}\right)\right)\right] d \boldsymbol{r}^{\prime}
$$




\section{Equations of motion}

The SPH particles are moved in phase space according to Eulerian equations

$$
\frac{d \boldsymbol{r}_{i}}{d t}=\boldsymbol{v}_{i}
$$

and

$$
\frac{d \boldsymbol{v}_{i}}{d t}=-\frac{1}{\rho_{i}} \nabla P_{i}+\boldsymbol{a}_{i}^{\mathrm{visc}}-\nabla \Phi_{i}
$$

where $\Phi_{i}$ is the gravitational potential, $P_{i}$ is the pressure, and $\boldsymbol{a}_{i}^{\mathrm{visc}}$ is acceleration owing to artificial viscosity. The continuity equation

$$
\rho_{i}=\sum_{j} m_{j} W_{i j}
$$

is satisfied automatically to order $\mathcal{O}\left(h^{2}\right)$. The introduction of an artificial viscosity in Eq. 4.14 allows the presence of shocks in the flow. The pressure gradient term $(\nabla P) / \rho$ in Eq. 4.14 is calculated by using the identity

$$
\frac{\nabla P}{\rho}=\frac{2 \sqrt{P} \nabla \sqrt{P}}{\rho}
$$

and using the smoothed estimates according to equation Eq. 4.12. For the symmetrised expression, which conserves both linear and angular momentum exactly, we obtain:

$$
-\frac{\nabla P_{i}}{\rho_{i}}=-\sum_{j} m_{j} \frac{\sqrt{P_{i} P_{j}}}{\rho_{i} \rho_{j}}\left[\nabla_{i} W\left(r_{i j}, h_{i}\right)+\nabla_{i} W\left(r_{i j}, h_{j}\right)\right]
$$

where $r_{i j}=\left|\boldsymbol{r}_{i}-\boldsymbol{r}_{j}\right|$ and $\sqrt{P_{i} P_{j}} / \rho_{i} \rho_{j}$ represents the geometric mean of $P / \rho^{2}$.

\section{Artificial viscosity}

For an accurate treatment of shocks the introduction of an artificial viscosity is required (e.g., Monaghan \& Gingold 1983). In this version of the code a form suggested by Hernquist \& Katz (1989) has been adopted,

$$
\boldsymbol{a}_{\mathrm{visc}}=-\sum_{j} m_{j} \Pi_{i j} \frac{1}{2}\left[\boldsymbol{\nabla}_{i} W\left(r_{i j}, h_{i}\right)+\nabla_{i} W\left(r_{i j}, h_{j}\right)\right]
$$

where

$$
\Pi_{i j}=\frac{-\alpha \bar{c}_{i j} \mu_{i j}+\beta \mu_{i j}^{2}}{\bar{\rho}_{i j}},
$$




$$
\mu_{i j}=\left\{\begin{array}{lll}
\frac{\mathbf{v}_{i j} \cdot \mathbf{r}_{i j}}{h_{i j}\left[\left(\frac{r_{i j}}{h_{i j}}\right)^{2}+\eta^{2}\right]} & \text { for } \quad \boldsymbol{v}_{i j} \cdot \boldsymbol{r}_{i j}<0 \\
0 & \text { for } \quad \boldsymbol{v}_{i j} \cdot \boldsymbol{r}_{i j} \geq 0
\end{array}\right.
$$

and $\bar{c}_{i j}=\left(c_{i}+c_{j}\right) / 2, \bar{h}_{i j}=\left(h_{i}+h_{j}\right) / 2, \bar{\rho}_{i j}=\left(\rho_{i}+\rho_{j}\right) / 2$. and $\mathbf{v}_{i j}=\boldsymbol{v}_{i}-\boldsymbol{v}_{j}$. We use the typical values for the constants $\alpha \sim 1$ and $\beta \sim 2$ (Hernquist \& Katz 1989; Evrard 1988). The first term in Eq. 4.19 is analogous to a bulk viscosity (e.g., Lucy 1977), whereas the second term is similar to a Von Neuman-Richtmyer (or molecular) artificial viscosity.

Artificial viscosities, however, can in general introduce considerable shear viscosity into the flow. To reduce the effective shear viscosity, we introduce a viscosity 'switch' (e.g., Benz 1990) in the form of a multiplicative factor $f_{i j}=\left(f_{i}+f_{j}\right) / 2$ on $\mu_{i j}$, where

$$
f_{i}=\frac{|\boldsymbol{\nabla} \cdot \boldsymbol{c}|_{i}}{|\boldsymbol{\nabla} \cdot \boldsymbol{v}|_{i}+|\boldsymbol{\nabla} \times \boldsymbol{v}|_{i}+0.001 c_{i} / h_{i}},
$$

and the curl of the velocity is given by the estimate

$$
\boldsymbol{\nabla} \times \boldsymbol{v}=\frac{1}{\rho_{i}} \sum_{j} m_{j} \boldsymbol{v}_{i j} \times \nabla W_{i j}
$$

\section{Thermal equation}

Using the first law of thermodynamics, we obtain the time evolution of the internal energy $u$ :

$$
d u=-P d V+T d s,
$$

where $V=1 / \rho$ and $s$ is the specific entropy. The thermal equation is then calculated from:

$$
\frac{d u_{i}}{d t}=\sum_{j} m_{j}\left(\frac{\sqrt{P_{i} P_{j}}}{\rho_{i} \rho_{j}}+\frac{1}{2} \Pi_{i j}\right) \boldsymbol{v}_{i j} \cdot \frac{1}{2}\left[\nabla_{i} W\left(r_{i j}, h_{i}\right)+\nabla_{i} W\left(r_{i j}, h_{j}\right)\right]
$$

The non-adiabatic effect of the artificial viscosity is included in the change of the specific entropy.

\section{Equation of state}

In order to close the system of equations an equation of state is needed. In this work we use the polytropic relation

$$
P=K \rho^{\gamma}
$$

This reduces to the isothermal relation, which has been used throughout this work, with $K=c_{\mathrm{s}}^{2}$ and $\gamma=1$. 


\subsubsection{Integration and time-stepping}

To integrate the equations of motion there exist different numerical schemes (e.g., Press et al. 1986), each with its own advantages and disadvantages. In the code used in this work we apply two different types of numerical integrators: a leapfrog integrator for the stellar components and a predictor/corrector method for the gas.

One of the advantages of the leapfrog integrator compared to other integrators is, that it is time reversible because of the symmetric way in which it is defined. This is important, for systems where energy conserved. Furthermore the leapfrog integrator is computationally less expensive than, for example, the predictor/corrector approach, and it requires less storage. This could be an important advantage in the case of large $N$ calculations. Moreover it is a very stable method, an advantage when the forces are only approximated.

For the gas integration a predictor corrector method is employed. This is advantages for the viscosity computation as positions and velocities are always synchronized.

\section{Hierachical time-steps}

The individual time-step for the collisionless (stellar) particles is defined by

$$
\delta t_{i}=\sqrt{\frac{\eta\left|\boldsymbol{a}_{i}\right|}{\left|\ddot{\boldsymbol{a}}_{i}\right|}},
$$

where $\boldsymbol{a}_{i}$ and $\eta$ are the acceleration on the particle and a dimensionless accuracy parameter, respectively. For a appropriate estimate of ä see Heller (1991). For the dissipative particles, i.e. the gas, we include the Courant condition and the condition demanded by the diffusive terms, by:

$$
\delta t_{i}=C \min \left(\sqrt{\frac{h_{i}}{\left|\boldsymbol{a}_{i}\right|}} ; \frac{h_{i}}{\sigma_{i}}\right),
$$

where $C \approx 0.3$ is the Courant number, $h_{i}$ the smoothing length and $\sigma_{i}$ is the viscosity parameter defined in Hernquist \& Katz (1989) by:

$$
\sigma_{i}= \begin{cases}h_{i}\left|\boldsymbol{\nabla} \cdot \boldsymbol{v}_{i}\right|+c_{i}+1.2\left(\alpha c_{i}+\beta h_{i}\left|\boldsymbol{\nabla} \cdot \boldsymbol{v}_{i}\right|\right) & \boldsymbol{\nabla} \cdot \boldsymbol{v}_{i}<0 \\ h_{i}\left|\boldsymbol{\nabla} \cdot \boldsymbol{v}_{i}\right|+c_{i}+1.2 \alpha c_{i} & \boldsymbol{\nabla} \cdot \boldsymbol{v}_{i} \geq 0\end{cases}
$$

To cover the wide dynamical range, i.e. some three to four orders of magnitude in radius and density, we advance the system with a fixed set of hierarchical timesteps (Hernquist \& Katz 1989, Whitehurst 1988). The multiple time-steps or bins are defined by:

$$
\Delta t_{k}=\frac{\Delta t_{0}}{2^{i}}, \text { with } k=1,2, \ldots, n
$$




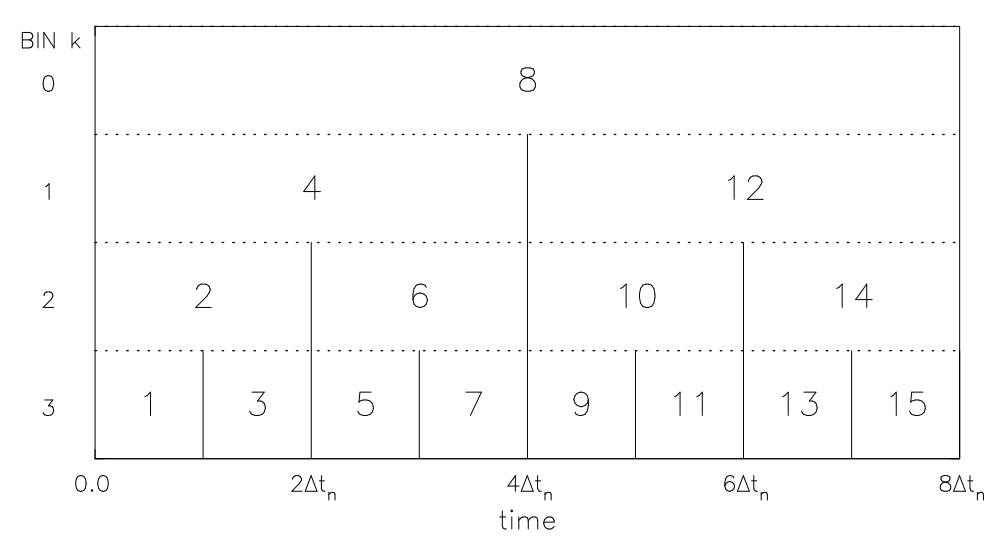

Figure 4.2: Schematic illustration of the time-stepping scheme. The bin number $k$ is represented by the vertical axis. The horizontal axis represents the time in units of $\Delta t_{n}$. Each box represents the advancement of a bin in time with the numbers specifying the order in which the steps are made.

where the number of sub-bins $k$ and the maximum time-bin $\Delta t_{0}$ remain fixed during the simulation. Individual particles are then placed in the bins according to their individually computed step sizes (Eq. 4.26 and 4.27).

The bins for the particles are selected according to the scheme showed in Fig. 4.2, and all particles in a particular time-bin are advanced together. Using this scheme the entire system will be time synchronised only after each set of $\left(2^{n+1}-1\right)$ timesteps. We allow an individual particle to move to a smaller time-bin, i.e. to larger $k$, at the end of its step and to a larger time-bin, i.e. to smaller $k$, when both are time synchronised. All relevant quantities are extrapolated to the mid-point time as required by the numerical integrator and thus it is never necessary to extrapolate a particle in time greater than half its current time-step $\delta t_{i}$. 


\subsection{Setting up the models}

In this section we describe how the $N$-body models used throughout this work are initially set up from a given analytic potential or density distribution. The realisation of the multicomponent systems, i.e. the equilibrium disc-halo models, follows basically the set-up procedure described in Barnes (1988).

\subsubsection{Plummer model}

For the density profile of the spherical components of the galaxies, e.g. halo or companion, we use a Plummer profile (Plummer 1911), i.e. a profile corresponding to a stellar polytrope of index 5 , which is given by:

$$
\rho_{\mathrm{Pl}}(r)=\frac{3}{4 \pi} M_{\mathrm{Pl}} b_{\mathrm{Pl}}^{2} \cdot\left(r^{2}+b_{\mathrm{Pl}}^{2}\right)^{-5 / 2},
$$

where $M_{\mathrm{Pl}}$ and $b_{\mathrm{Pl}}$ are the total mass and the radial scalelength of the sphere, respectively. This distribution was originally used by Plummer to fit observations of globular clusters, but is also used for describing stellar models and quasi-spherical components like galactic bulges and halos. In Fig. 4.3 we show an example of the volume density of a Plummer sphere as a function of radius.

Using Poisson's equation (Eq. 3.4) the corresponding gravitational potential of the Plummer sphere is given by:

$$
\Phi_{\mathrm{Pl}}(r)=-\mathrm{G} M_{\mathrm{Pl}}\left(r^{2}+b_{\mathrm{Pl}}^{2}\right)^{-1 / 2},
$$

where $\mathrm{G}$ is the gravitational constant. The potential energy of the Plummer sphere is

$$
W=-4 \pi \mathrm{G} \int_{0}^{\infty} \Phi(r) \rho(r) r d r=-\frac{3 \pi}{32} \mathrm{G} M_{\mathrm{Pl}}^{2} b_{\mathrm{Pl}}^{-1}
$$

With the assumptions that the system is in a steady state and the velocity distribution is isotropic, the corresponding distribution function can directly be derived from the density distribution following the description in Binney \& Tremaine (1987) by using Eddington's formula (Eddington 1916):

$$
f(E)=\frac{1}{\sqrt{8} \pi^{2}} \frac{d}{d E} \int_{0}^{E} \frac{d \rho}{d \Phi} \frac{d \Phi}{\sqrt{E-\Phi}},
$$

where $E$ is the specific energy $E=\Phi+v^{2} / 2$ of a particle. The resulting distribution function of a Plummer sphere is then given by:

$$
f(\mathbf{r}, \mathbf{v})=\left\{\begin{array}{cc}
\frac{24 \sqrt{2}}{7 \pi^{3}} \mathrm{G}^{-5} M_{\mathrm{Pl}}^{-4} b_{\mathrm{Pl}}(-E)^{7 / 2} & : \quad E<0 \\
0 & : \quad E \geq 0 .
\end{array}\right.
$$




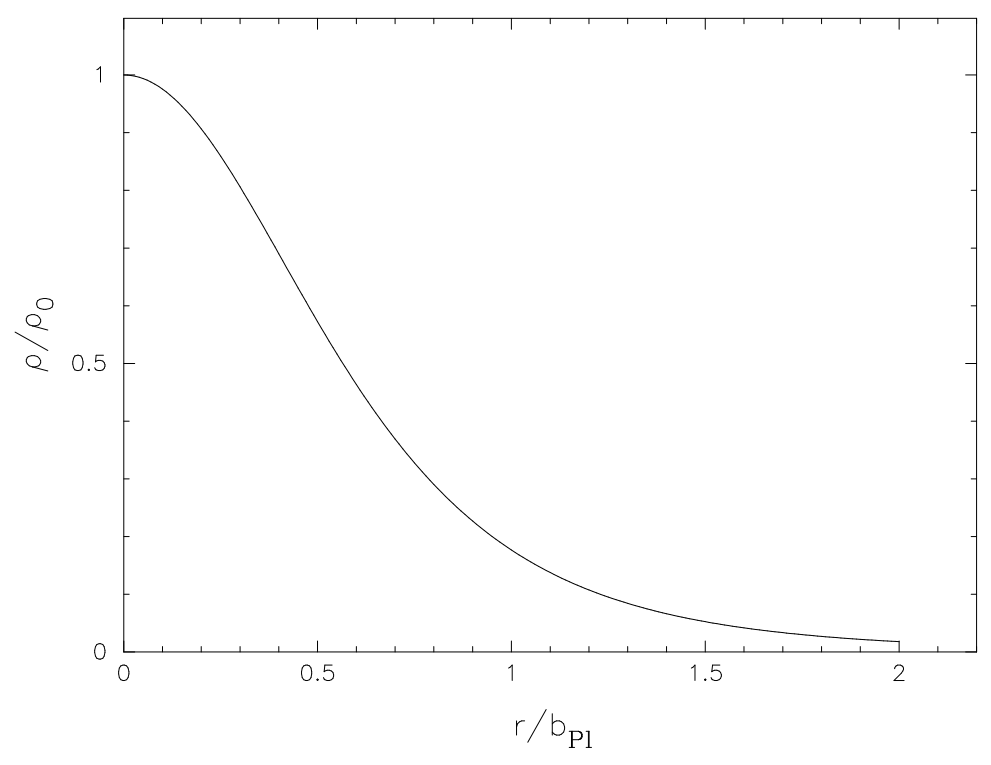

Figure 4.3: Normalised volume density of a Plummer sphere. Both the density and the length scales are normalised by the central density $\rho_{0}$ and the radial scalelength $b_{\mathrm{Pl}}$, respectively.

The $k$-th moment of the velocity distribution is obtained by integration of the above equation over the whole velocity space:

$$
\left\langle v^{k}(\boldsymbol{r})\right\rangle=\int v^{k} f(\boldsymbol{r}, \boldsymbol{v}) d \boldsymbol{v}
$$

The velocity dispersion is the second moment $(k=2)$ of the distribution function. A detailed description of the Plummer model and its numerical $N$-body realisation is given by Aarseth et al. (1974).

\section{Truncated model}

The Plummer model has a finite total mass and a non-zero density everywhere. In the $N$-body realisation, however, the system is truncated at some given cut-off radius $r_{\text {cut }}$ and the velocity distribution needs to be corrected accordingly to keep the model in virial equilibrium. The cut-off mass $M_{\text {cut }}$ is given by:

$$
M_{\text {cut }} \equiv M\left(r_{\text {cut }}\right)=M_{\mathrm{Pl}} \cdot r_{\text {cut }}^{3} \cdot\left(r_{\text {cut }}^{2}+b_{\mathrm{Pl}}^{2}\right)^{-3 / 2} .
$$

The derivation of the velocities assumes an infinite smooth sphere, which underestimates them in the truncated, discrete model. Therefore we correct the assigned 
velocities by some factor $c_{0}$ directly calculated from the $N$-body model. Virial equilibrium requires:

$$
2 E+W=\sum_{i=1}^{N} m_{i}\left\langle c_{0} \cdot v_{i}^{2}\right\rangle+W \equiv 0,
$$

where $v_{i}$ is the velocity obtained from the infinite distribution. The factor $c_{0}$ is then given by

$$
c_{0}=\sqrt{\frac{|W|}{2 E}} .
$$

\subsubsection{Kuzmin-Toomre disc}

For the stellar and gaseous disc component we use a radial surface density of the Kuzmin-Toomre model (Kuzmin 1956; Toomre 1963; see also Binney \& Tremaine 1987), which is given by:

$$
\Sigma_{\mathrm{KT}}(R)=\frac{1}{2 \pi} a_{\mathrm{KT}} M_{\mathrm{KT}}\left(R^{2}+a_{\mathrm{KT}}^{2}\right)^{-3 / 2},
$$

where $M_{\mathrm{KT}}$ and $a$ are the total mass and radial scalelength of the disc, respectively. In Fig. 4.4 (upper panel) we show an example of the radial surface density of a Kuzmin-Toomre disc. The radial mass distribution of the disc is given by:

$$
M(R)=M_{\mathrm{KT}} \cdot\left[1-a_{\mathrm{KT}} \cdot\left(R^{2}+a_{\mathrm{KT}}^{2}\right)^{-1 / 2}\right]
$$

For the truncated $N$-body model the mass within the cut-off radius $R_{\text {cut }}$ is given by

$$
M_{\mathrm{cut}} \equiv M_{\mathrm{KT}}\left(R_{\mathrm{cut}}\right)=M_{\mathrm{KT}} \cdot\left[1-a_{\mathrm{KT}} \cdot\left(R_{\mathrm{cut}}^{2}+a_{\mathrm{KT}}^{2}\right)^{-1 / 2}\right] .
$$

The vertical density distribution of the disc in our models follows that of an isothermal sheet (Spitzer 1942), given by:

$$
\Sigma(z)=\Sigma_{0}(R) \cdot \operatorname{sech}^{2}\left(z / z_{0}\right)
$$

where $\Sigma_{0}(R)$ and $z_{0}$ are the central surface density and the vertical scaleheight of the disc, respectively. The mass within $\pm z$ is then given by

$$
M_{\mathrm{KT}}(z)=2 \Sigma_{0} \cdot z_{0} \cdot \tanh \left(z / z_{0}\right)
$$

Before we assign the velocities to the disc particles in a self-consistent way, we first have to relax the halo on the frozen disk, i.e. that the disc particles keep their initial positions with respect to each other during the relaxation process. 


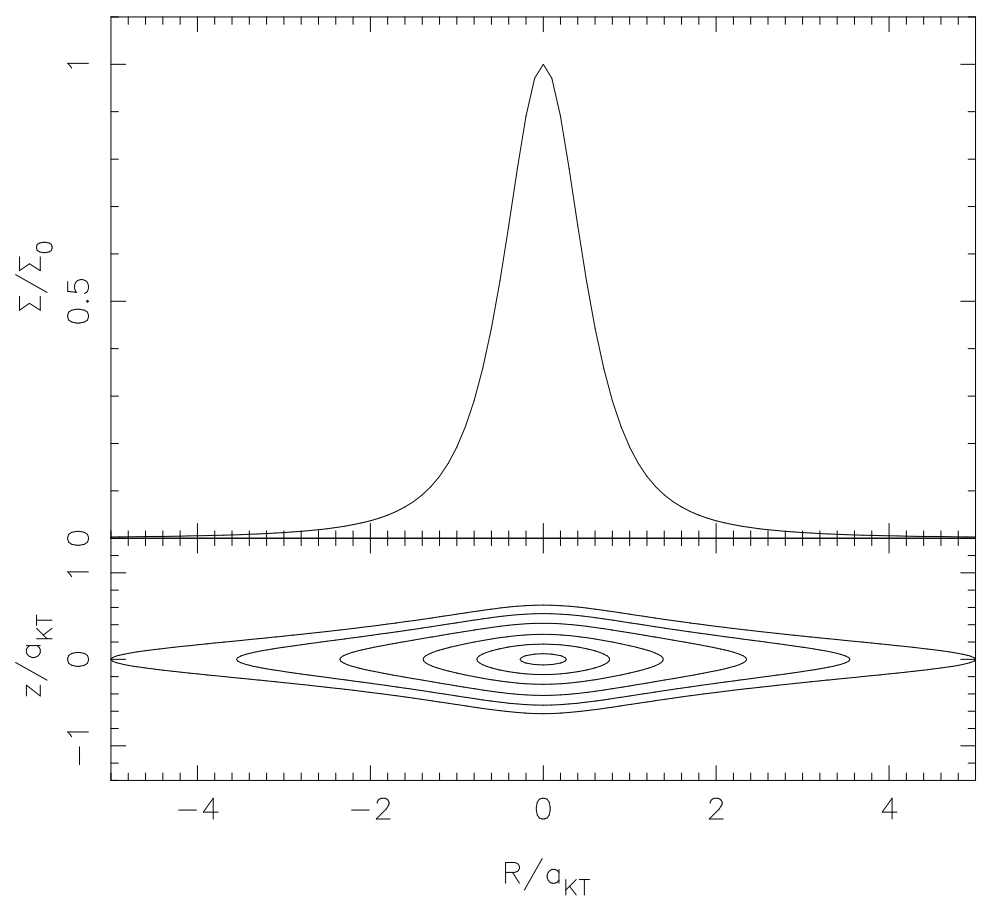

Figure 4.4: Density distribution of a Kuzmin-Toomre disc. The top panel shows the normalized surface density. The bottom panel shows isodensity contours of an isothermal sheet.

\subsubsection{Relaxation of the halo}

The set-up procedure of the multicomponent system basically follows the description of Barnes (1988). The initially spherical halo is set up such as to be in virial equilibrium. This equilibrium is disturbed by adding the disc to the halo owing to the extra gravitational potential. The halo is therefore allowed to relax by evolving it in the gravitational potential of the frozen disc. To reduce the effects of violent relaxation the gravitational field of the disc is gradually imposed over some timescale $t_{0}$, while the halo is dynamically evolved and responds quasi-adiabatically, e.g. the potential variations are slow compared to the typical orbital frequencies in the halo. During the relaxation the disc potential is directly calculated from the $N$-body model with the particle masses of the disc being slowly increased by some factor $u(\tau)$, following a normalised spline function of the form

$$
u(\tau)=\left\{\begin{array}{cc}
(3-2 \tau) \cdot \tau^{2} & : \quad \tau \leq 1 \\
1 & : \quad \tau>1
\end{array}\right.
$$

where $\tau=t / t_{0}$. In Fig. 4.5 we show the response of the halo to the slowly increased disc. In the models presented in this work we typically use $t_{0}=60 \approx 12 \tau_{\text {dyn }}$. The halo is evolved for another $\Delta t=60$ after the disc has reached its full mass. 


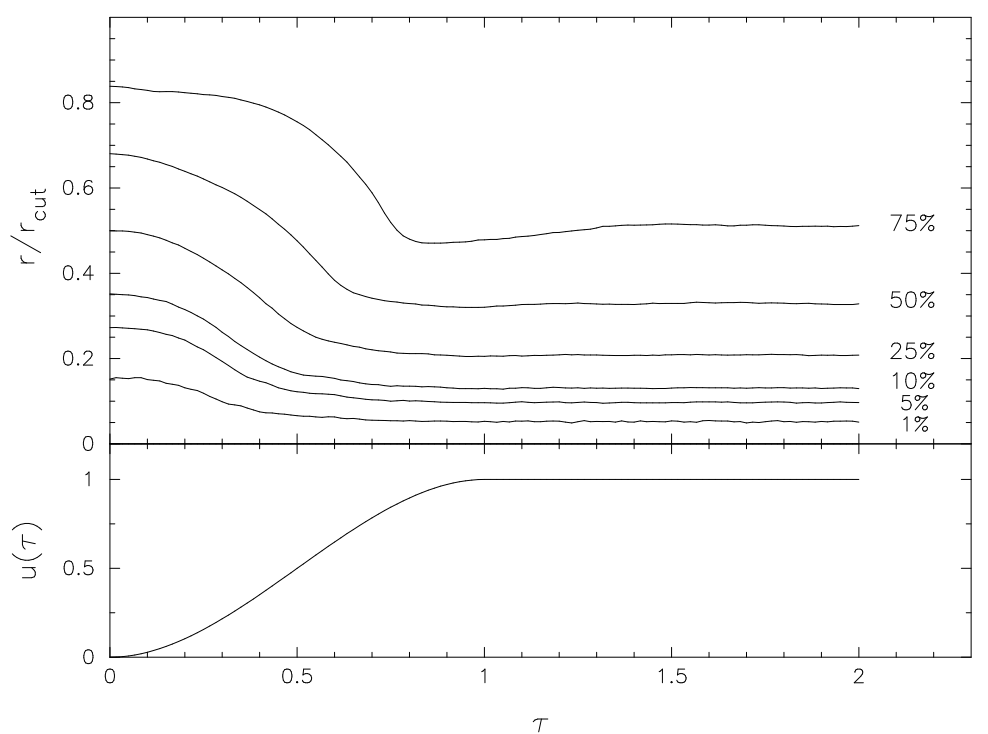

Figure 4.5: Relaxation of the halo. The upper panel shows radii of constant mass normalised by the initial cut-off radius $r_{\text {cut }}$ of the halo as a function of $\tau$. In the bottom panel we show the function $u(\tau)$ used for gradually increasing the disc's mass.

\subsubsection{Assigning the disc velocities}

After the halo has reached a new virial equilibrium after its relaxation, we now assign the velocities to the disc particles. We first calculate the azimuthally averaged gravitational potential of the galaxy (halo+disc) on a polar grid in the equatorial plane of the disc. The rotation curve $v_{\mathrm{c}}(R)$ of the disc is then derived from the potential using Eq. 3.11. Thus we can derive the principal circular velocity $v_{\mathrm{c}}$ for each disc particle by interpolation. The radial velocity dispersion $\sigma_{R}$ of the disc particles have been chosen so that the $Q$-parameter (Toomre 1964):

$$
\sigma_{R}=3.358 \frac{1}{\kappa} \Sigma(R) \cdot Q
$$

is independent of radius. We have calculated the tangential velocity dispersion using the relation (see, for example, Binney \& Tremaine 1987):

$$
\sigma_{\phi}=\frac{1}{2} \cdot \frac{\sigma_{R} \kappa}{\Omega_{\mathrm{c}}} .
$$

Finally we assign the vertical velocity dispersion $\sigma_{z}$ of an isothermal sheet given by:

$$
\sigma_{z}=\sqrt{\pi \mathrm{G} \Sigma z_{0}} .
$$




\section{Asymmetric drift correction}

Since the surface density of stars in the disc in general decreases with increasing radius, the average azimuthal velocity (hereafter $\bar{v}_{\phi}$ ) of the stars in some volume element is smaller than the principal circular velocity $v_{\mathrm{c}}$, i.e. that the net motion of the stars lags behind the local circular velocity. This effect is called the asymmetric drift. It increases with increasing average random motion, or velocity dispersion, of the stars.

To correct for the asymmetric drift in our models, the velocities of the stellar disc particles have been corrected following the description of Binney \& Tremaine (1987). The asymmetric drift $v_{\mathrm{a}}$ is defined as:

$$
v_{\mathrm{a}} \equiv v_{\mathrm{c}}-\bar{v}_{\phi}
$$

To derive the relationship for the asymmetric drift, we take the radial velocity moment of the collisionless Boltzmann equation (Eq. 3.3) for a steady-state axisymmetric system in cylindrical coordinates. By multiplying this equation with the radial velocity component $v_{R}$ and integrating over all velocities we obtain the Jeans equation:

$$
\frac{R}{\Sigma} \frac{\partial\left(\Sigma \overline{v_{R}^{2}}\right)}{\partial R}+R \frac{\partial\left(\overline{v_{R} v_{z}}\right)}{\partial z}+\overline{v_{R}^{2}}-\overline{v_{\phi}^{2}}+R \frac{\partial \Phi}{\partial R}=0 \quad(\text { for } z=0)
$$

With the definition of the azimuthal velocity dispersion

$$
\sigma_{\phi}^{2}=\overline{\left(v_{\phi}-\bar{v}_{\phi}\right)^{2}}=\overline{v_{\phi}^{2}}-\bar{v}_{\phi}^{2}
$$

and substitution of $R(\partial \Phi / \partial R)=v_{\mathrm{c}}^{2}$ we obtain from Eq. 4.49:

$$
\begin{aligned}
\sigma_{\phi}^{2}-\overline{v_{R}^{2}}-\frac{R}{\Sigma} \frac{\partial\left(\Sigma \overline{v_{R}^{2}}\right)}{\partial R}-R \frac{\partial\left(\overline{v_{R} v_{z}}\right)}{\partial z} & =v_{\mathrm{c}}^{2}-\bar{v}_{\phi}^{2} \\
& =\left(v_{\mathrm{c}}-\bar{v}_{\phi}\right)\left(v_{\mathrm{c}}+\bar{v}_{\phi}\right) \\
& =v_{\mathrm{a}}\left(2 v_{\mathrm{c}}-v_{\mathrm{a}}\right)
\end{aligned}
$$

With the assumption that the asymmetric drift $v_{\mathrm{a}}$ is small compared to $2 v_{\mathrm{c}}$ we obtain the following expression:

$$
2 v_{\mathrm{c}} v_{\mathrm{a}} \simeq \overline{v_{R}^{2}}\left[\frac{\sigma_{\phi}^{2}}{\overline{\overline{v_{R}^{2}}}}-1-\frac{\partial \ln \left(\Sigma \bar{v}_{R}^{2}\right)}{\partial \ln R}-\frac{R}{\overline{v_{R}^{2}}} \frac{\partial\left(\overline{v_{R} v_{z}}\right)}{\partial z}\right]
$$

Observations of external galaxies (van der Kruit \& Searle 1981; van der Kruit \& Freeman 1984) suggest that in these system $\overline{v_{R}^{2}}$ is roughly proportional to $\Sigma$. Assuming that the shape of the velocity ellipsoid is constant, we estimate that $\left[\partial \ln \left(\Sigma \overline{v_{R}^{2}}\right)\right] / \partial \ln R \simeq 2(\partial \ln \Sigma / \partial \ln R)$. The value of the other derivative depends on the orientation of the velocity ellipsoid at points just above the plane of a galaxy. 


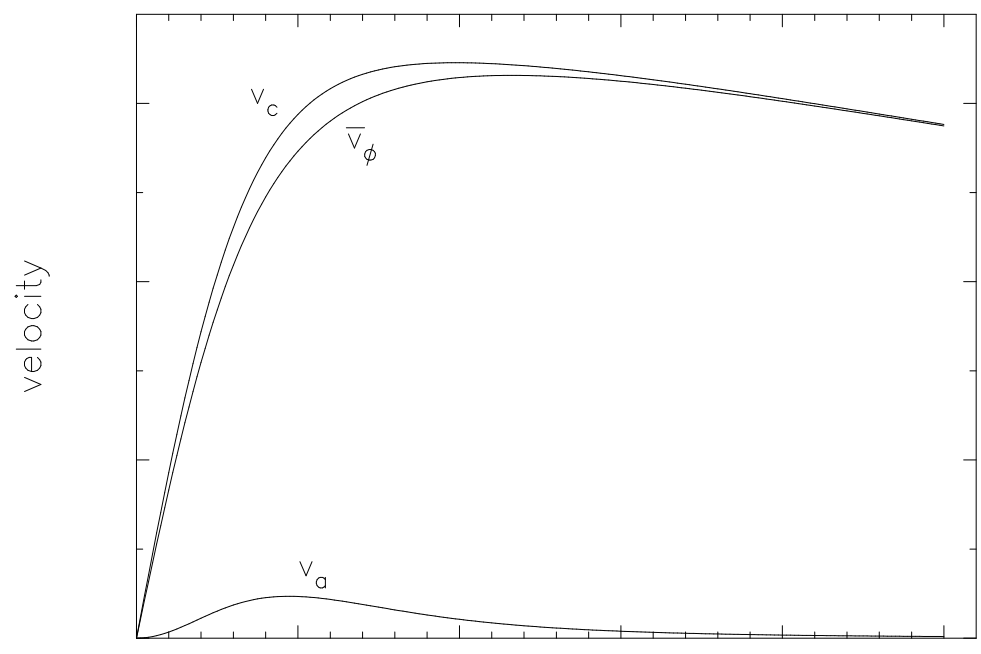

radius

Figure 4.6: Asymmetric drift correction. The plot shows the local circular velocity $v_{c}$, the average azimuthal motion $\bar{v}_{\Phi}$, and the asymmetric drift term $v_{a}$ as a function of radius.

For simplicity we assume that the ellipsoid's principal axes remains aligned with the coordinate directions of the $(R, \phi, z)$ system, e.g. $\left(\overline{v_{R} v_{z}}\right)$ is independent of $z$. We have therefore

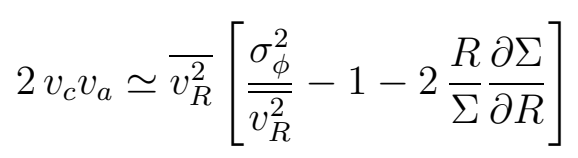

The case of greatest practical importance is when the system is in a steady state and $\bar{v}_{r}=\bar{v}_{\phi}=0$, since there is no net streaming in the radial component of the velocity dispersion, i.e.

$$
\sigma_{R}^{2} \equiv \overline{v_{R}^{2}}-\bar{v}_{R}^{2}=\overline{v_{R}^{2}}
$$

If the asymmetric drift of the population under study is small, we have $\bar{v}_{\phi} \simeq v_{\mathrm{c}}$ and finally get

$$
\begin{aligned}
v_{\mathrm{c}}^{2}-\bar{v}_{\phi}^{2} & =2 v_{\mathrm{c}} v_{\mathrm{a}} \\
& \simeq \sigma_{R}^{2}\left[\frac{\sigma_{\phi}^{2}}{\sigma_{R}^{2}}-1-2 \frac{R}{\Sigma} \frac{\partial \Sigma}{\partial R}\right]
\end{aligned}
$$




\section{Application: Kuzmin-Toomre disc}

With the surface density of a Kuzmin-Toomre disc,

$$
\frac{\partial \Sigma_{\mathrm{KT}}}{\partial R}=-\frac{3 R \Sigma_{\mathrm{KT}}(R)}{R^{2}+a_{\mathrm{KT}}^{2}}
$$

and using Eq. 4.46, we finally obtain the mean tangential velocities for the disc particles as:

$$
\bar{v}_{\phi}^{2}=v_{\mathrm{c}}^{2}-\sigma_{R}^{2}\left[\frac{\kappa}{4 \Omega_{\mathrm{c}}}-1+6 R^{2}\left(R^{2}+a_{\mathrm{KT}}^{2}\right)^{-1}\right]
$$

In Fig. 4.6 we show an example of the asymmetric drift correction in a KuzminToomre disc.

\subsubsection{Including the companion}

To create the interaction models, the companion galaxy has to be introduced in the initial model. The simulations presented in this work require specific initial conditions for the companion, i.e. the impact position of the companion or pericentric position with respect to the phase angle of the rotating bar. To create the initial centre of mass positions and velocities of both the host and the companion galaxy we use the solution of the corresponding two-body problem. For large distances this approximation is valid, but with close encounters the local potential of the galaxies become more important. Therefore we introduce the companion to the model at pericentric time (hereafter $t_{\text {peri }}$ ) with given initial velocities and integrate their orbits backward in time with a frozen disc. We will first summarise the results of the Keplerian two-body problem to determine the centre of mass positions and velocities and then describe the set-up procedure for the different types of interactions studied in this work.

\section{The two-body problem}

The classical two-body problem can be reduced to the equations of motion of a particle with a reduced mass

$$
\mu=\frac{M_{1} \cdot M_{2}}{M_{1}+M_{2}}
$$

where $M_{1}$ and $M_{2}$ are the mass of - in our case - the two galaxies, moving in a central potential of the form:

$$
V(r)=-\frac{\mathrm{G} M_{1} M_{2}}{r} \equiv-\frac{\alpha}{r},
$$




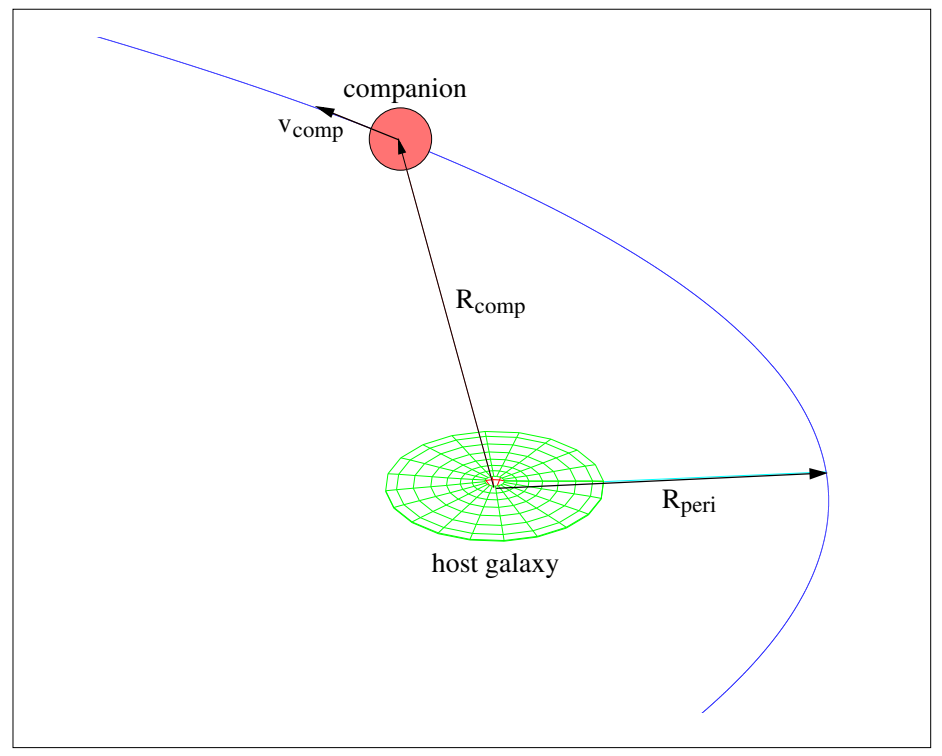

Figure 4.7: Schematic illustration of the galaxy-galaxy encounter in the frame of reference of the host galaxy. The orbit of the companion galaxy lies in the equatorial plane of the host disc.

with $\alpha=\mathrm{G} M_{1} \cdot M_{2}$. Since the angular momentum $L$ is a conserved quantity the motion of the particle is confined to a fixed plane and we can define an effective potential energy of the particle as (in polar coordinates):

$$
V_{\text {eff }}(R)=-\frac{\alpha}{R}+\frac{L^{2}}{2 \mu R^{2}}
$$

where $L=\mu R^{2} \dot{\phi}$. The effective potential $V_{\text {eff }}$ has a minimum at $R=L^{2} /(\mu \alpha)$ with a value of $V_{\text {eff }}^{\min }=-\mu \alpha^{2} / 2 L^{2}$.

In practice we would like to derive the initial conditions of the galaxies for an orbit with a given eccentricity $e$ and pericentric separation $R_{\text {peri }}$. By solving the corresponding Hamiltonian the principal path is given in a parametrised form by:

$$
\phi=\cos ^{-1}\left(\frac{(L / R)-(\mu \alpha / L)}{\sqrt{\left(2 \mu E+\mu^{2} \alpha^{2} / L^{2}\right.}}\right)+\text { const. }
$$

where $E$ is the total energy, given by:

$$
E=V_{\text {eff }}+\frac{1}{2} \mu \dot{R}^{2}
$$


The coordinate system can always be chosen such that const $\equiv 0$. With $p=\frac{L^{2}}{\mu \alpha}$ and the orbits eccentricity $e=\sqrt{\left[1+\left(2 E L^{2} / m \alpha^{2}\right)\right]}$ we can write Eq. 4.61 as:

$$
\frac{p}{R}=1+e \cos \phi
$$

If we choose $\phi_{\text {peri }} \equiv \phi\left(R_{\text {peri }}\right)=0$ for a pericentric separation $R_{\text {peri }}$, we can calculate the angular momentum for a given orbit with eccentricity $e$ from Eq. 4.63 as:

$$
L^{2}=\mu \alpha R_{\text {peri }}(1+e)
$$

From the conservation of angular momentum $L=\mu R^{2} \dot{\phi}=$ const. we finally get

$$
\dot{\phi}\left(t_{\mathrm{peri}}\right)=\frac{L}{\mu R_{\mathrm{peri}}^{2}}=\frac{\sqrt{\mu \alpha R_{\mathrm{peri}}(1+e)}}{\mu R_{\mathrm{peri}}^{2}}
$$

In a similar way the initial conditions are obtained for given $\dot{\phi}\left(t_{\text {peri }}\right)$ and $r_{\text {peri }}$.

\section{Vertical impacts}

The initial conditions of the companion and the host in the simulations presented in Chap. 5 are obtained as follows. After selecting the impact position in the disc, the initial centre of mass positions and velocities of the host and companion were obtained by integrating their orbits backward in time, starting from the impact time $t_{\text {imp }}$ at which the centre of mass of the companion lies in the disc plane. During this integration the disc (with bar) and halo particles of the host galaxy are frozen with respect to each other, but allowed to rotate as a single system with the (negative) angular frequency or pattern speed $\Omega_{\mathrm{p}}(t)$ of the bar, as determined from the isolated disc model. The companion is represented by a point mass for this integration. This approximation is sufficient since the encounter is fast enough that dynamical friction does not significantly modify the orbit.

\section{Tidal encounters}

In the simulations presented in Chap. 6 we confine ourselves to planar fly-by encounters, i.e. the orbits of the host and the companion galaxy are unbound and lie in the equatorial plane of the host. The orbit of the companion galaxy is prograde with respect to the rotation in the host disc and has been chosen to be either parabolic or hyperbolic, with different pericentric separations $r_{\text {peri }}$. The pericentric position has been chosen such that at time of pericentre (hereafter $t_{\text {peri }}$ ) the major axis of the bar, which has formed/evolved in the hosts disc before the encounter, points in the direction of the companion. The initial orbital parameters at $t_{\text {peri }}$ were then calculated from the solution of the corresponding two-body problem either for a given orbital eccentricity $e$ or for a pericentric angular frequency of the companion

$$
\dot{\phi} \equiv \omega_{\text {peri }}=v_{\text {peri }} / R_{\text {peri }}
$$


where $v_{\text {peri }}$ is the velocity of the companion at pericentre. To obtain the initial centre of mass positions and velocities of the host and of the companion galaxy, we integrate their orbits backward in time, starting from the pericentric time $t_{\text {peri }}$, until the distance $\Delta R$ between host and companion is reached. During the initial orbit integration the particles of the host are - as before - frozen with respect to each other. 


\section{Results}



5. Numerical simulations of interacting gas-rich barred galaxies: vertical impact of small companions. ${ }^{1}$

\author{
I. Berentzen ${ }^{1,2}$, E. Athanassoula ${ }^{2}$, C.H. Heller ${ }^{3}$, and K.J. Fricke ${ }^{1}$ \\ ${ }^{1}$ Universitäts-Sternwarte, Geismarlandstraße 11, D-37083 Göttingen, Germany \\ ${ }^{2}$ Observatoire de Marseille, 2 Place Le Verrier, F-13248 Marseille Cedex 4, France \\ ${ }^{3}$ Georgia Southern University, Department of Physics, Statesboro, GA 30460, U.S.A.
}

\begin{abstract}
We investigate the dynamical effects of an interaction between an initially barred galaxy and a small spherical companion using an $N$-body/ smoothed-particle-hydrodynamics algorithm. In the models described here the small companion passes through the disc of the larger galaxy nearly perpendicular to its plane. The impact positions and times are varied with respect to the phase of the bar and the dynamical evolution of the disc.

The interactions produce expanding ring structures, off-set bars, spokes, and other asymmetries in the stars and gas. These characteristic signatures of the interaction are present in the disc for about $1 \mathrm{Gyr}$. We find that in some cases it is possible to destroy the bar while keeping the disc structure. In general, the central impacts cause larger damage to the bar and the disc than the peripheral ones. The interaction tends to accelerate the transition from a strongly-barred galaxy to a weaklyor non-barred galaxy. The final disc morphology is determined more by the impact position relative to the bar rather than the impact time.
\end{abstract}

\title{
5.1 Introduction
}

The evolution of disc galaxies is driven through both internal and external processes. Internal instabilities in the disc often give rise to the formation of a bar, which as many as half of all disc galaxies are now known to harbour. In the last few years near-infrared (NIR) observations (Pompea \& Rieke 1990; Rix 1993; Rix \&

\footnotetext{
${ }^{1}$ This paper has been published in Monthly Notices of the Royal Astronomical Society 341, 343-360 (2003).
} 
Rieke 1993; Rix \& Rieke 1993; Seigar \& James 1998; Eskridge et al. 2000) have confirmed that bars are a very common feature in disc galaxies and by no means an exception. The presence of a bar changes both the kinematics and mass (stellar and gas) distributions within the disc and can give raise to dynamical resonances (see, for example, Sellwood \& Wilkinson 1993). Likewise, an only recently recognized important external agent is the interaction with small companions. A statistical survey by Zaritsky et al. $(1993,1997)$ gave a lower limit to the average number of companions for field spiral galaxies of $1.2 \pm 0.3$. Since these two processes are thought to be so common, it is a reasonable assumption that the interaction between a barred disc galaxy and a small companion would be a common event.

Although it is only a small fraction of the mass of a galaxy, gas can significantly influence the evolution of disc galaxies (see, for example, Shlosman \& Noguchi 1993). Owing to the torques of a stellar bar, the dissipative gas can be driven towards the galactic centre and channelled toward the inner kpc (Shlosman, Begelman \& Frank 1990; Athanassoula 1992b) driving active galactic nuclei (AGN) or nuclear starbursts (Heller \& Shlosman 1994) and creating nuclear and circumnuclear discs and rings. Recent NIR-observations (Pèrez-Ramírez et al. 2000) have shown a relation between the circumnuclear star formation in barred galaxies and the circumnuclear spiral or ring structure present in these galaxies. The shape of dust lanes inside the bar, coinciding with the location of shocks in the gasflow, reveals information about the internal dynamics of the bar and disc (Athanassoula 1992b). Many numerical simulations including a dissipative disc component have emphasized the importance of the gas in isolated barred galaxies (see, for example, Friedli \& Benz 1993; Shlosman \& Noguchi 1993; Heller \& Shlosman 1994; Berentzen et al. 1998) and interacting galaxies as well (see, for example, Noguchi 1987; Hernquist \& Weil 1993; Barnes \& Hernquist 1996).

Since the pioneering work of Toomre \& Toomre (1972) much effort has been spent to investigate galaxy-galaxy interactions (see, for example, the review by Barnes \& Hernquist 1992, and references therein). Noguchi (1988) started with numerical simulations to study the formation of bars in tidal interactions and the response of the gas to a tidal bar. Gerin, Combes \& Athanassoula (1990) have performed both 2D and 3D $N$-body simulations to study the response of an initial bar formed in a globally unstable disc (Ostriker \& Peebles 1973) to a tidal encounter with a spherical perturber. They studied the effect of a close encounter, both in and out of plane, with trajectories with pericentres outside the disc. This work has been continued by Sundin \& Sundelius (1991) and Sundin, Donner \& Sundelius (1993), who, using 2D $N$-body simulations, studied the response of a bar, which had been induced by a satellite on a planar parabolic orbit. In these works, the change in pattern speed, angular momentum distribution and resonances in the disc were investigated for different orbits and masses of the perturber. Further work, concentrating on the formation of collisionally induced rings, has been performed by Athanassoula, Puerari \& Bosma (1997, hereafter APB97). These purely collisionless simulations have shown that off-centred impacts of a sufficiently massive 
companion hitting the inner parts of a barred disc galaxy can displace the bar to one side, causing asymmetries and the formation of rings. We should note that off-centred bars may also form spontaneously in galaxies.

In this paper we focus upon the evolution of a gas-rich barred disc galaxy which is perturbed by the impact of a less massive spherical companion galaxy. An $N$ body/smoothed-particle-hydrodynamics ( $\mathrm{SPH}$ ) algorithm is used to evolve the stellar and gas components of the two systems. In the models described here the small companion passes through the disc of the larger galaxy nearly perpendicular to its plane. The impact position and time are varied with respect to the bar and to the evolutionary phase of the isolated barred galaxy. The mass of the companion galaxy has been chosen such that the interaction can be considered as a perturbation to the bar and disc. The set of encounter parameters has been deduced from the previous simulations by APB97 and chosen as to produce the strongest effects - such as asymmetries, off-centred bars and rings. We describe how the evolution of the bar strength, pattern speed, and gas inflow rate are affected by the interaction.

\subsection{Methods}

The method consists of an $N$-body algorithm to evolve the collisionless component (representing the stars and dark matter), combined with a smoothed particle hydrodynamics (SPH) algorithm to evolve the dissipative component (representing the gas) - see, for example, the review by Monaghan (1992). For the simulations presented in this paper we use the hybrid $N$-body/SPH code described in detail by Heller (1991) and Heller \& Shlosman (1994). The algorithm employs such features as a spatially varying smoothing length, a hierarchy of time bins to approximate individual particle time-steps, a viscosity 'switch' to reduce the effects of viscous shear, and the special purpose GRAPE-3AF hardware to compute the gravitational forces and neighbour interaction lists (Sugimoto et al. 1990; Steinmetz 1996). Besides speed in the direct force summation, the GRAPE hardware has the additional advantage that it does not impose any constraints on the spatial distribution of the particles.

The model of the disc galaxy (or host galaxy) is composed of a stellar and gaseous disc, embedded in a hot spherical dark matter halo. An isothermal equation of state is used for the gas component. The spherical companion galaxy (or perturber) consists of stars only.

\subsubsection{Initial conditions}

\section{Host galaxy and companion}

The model parameters for the host and the companion galaxy are selected similar to those used by APB97, so as to allow a meaningful comparison between the col- 


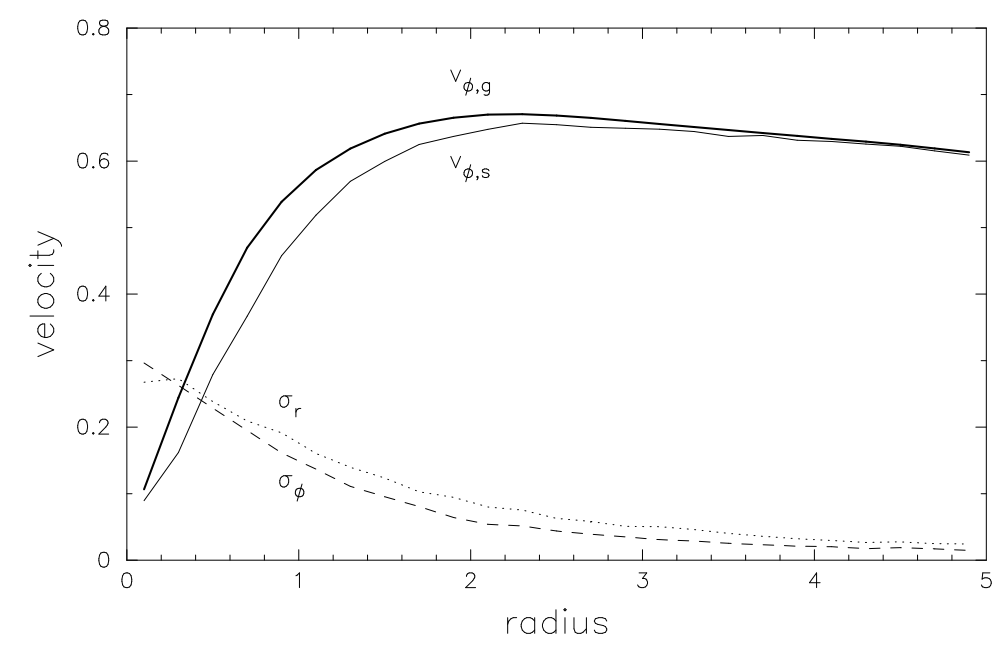

Figure 5.1: Initial velocity distribution of the host model $I 0$. We show the mean tangential velocity of both the stellar (thin full line) and the gaseous disc (thick full line) as a function of radius. The radial and tangential velocity dispersion of the stellar disc is shown with dotted and dashed lines, respectively.

lisionless and dissipative models. The host galaxy is a barred model referred to as $\mathbf{m b}$ in APB97. Our model here, however, differs from $\mathbf{m b}$ in that about 23 per cent of the mass of the stellar disc has been replaced by a gaseous disc. The radial scalelength and total mass of the disc have been retained in the model.

Both the stellar and the gaseous disc are initially setup with a Kuzmin-Toomre (hereafter KT) projected radial surface density profile (Kuzmin 1956; Toomre 1963)

$$
\Sigma_{\mathrm{KT}}(R)=\frac{1}{2 \pi} a M_{\mathrm{KT}} \times\left(R^{2}+a^{2}\right)^{-3 / 2} .
$$

In the above, $R, M_{\mathrm{KT}}$ and $a$ are the cylindrical radius, the total mass and the radial scalelength, respectively. The resulting truncated mass profile for the disc is then set up with the disc mass $M_{\mathrm{D}}=M_{\mathrm{s}}+M_{\mathrm{g}}$, where $M_{\mathrm{s}}$ and $M_{\mathrm{g}}$ are the masses of the stellar and the gaseous disc, respectively, which have been truncated at a radius $R_{\mathrm{D}}$. The vertical disc profile follows the $\operatorname{sech}^{2}\left(z / z_{i}\right)$ distribution of an isothermal sheet (Spitzer 1942), where $z_{i}$ is the vertical scaleheight with $i=\mathrm{s}$ and $i=\mathrm{g}$ for the stellar and gaseous disc, respectively.

Both the halo and the companion have a generic Plummer (hereafter Pl) density profile (Plummer 1911)

$$
\rho_{\mathrm{Pl}}(r)=\frac{3 b^{2} M_{\mathrm{Pl}}}{4 \pi}\left(r^{2}+b^{2}\right)^{-5 / 2},
$$

where $r$ is the spherical radius. $M_{\mathrm{Pl}}$ and $b$ denote the total mass and radial scalelength for each individual component. The truncated mass profile for the halo and 
Table 5.1: Initial model parameters.

\begin{tabular}{ccccccc}
\hline & Type & $\mathbf{N}$ & $\mathbf{M}_{\mathbf{D}}$ & $\mathbf{a}$ & $\mathbf{R}_{\mathbf{D}}$ & $\mathbf{z}_{\mathbf{s} / \mathbf{g}}$ \\
$\begin{array}{c}\text { Disc } \\
\text { stars }\end{array}$ & $\mathrm{KT}$ & 13500 & 0.54 & 1.0 & 5.0 & 0.20 \\
gas & $\mathrm{KT}$ & 10000 & 0.16 & 1.0 & 5.0 & 0.05 \\
& Type & $\mathbf{N}$ & $\mathbf{M}_{\mathbf{H}}$ & $\mathbf{b}_{\mathbf{H}}$ & $\mathbf{r}_{\mathbf{H}}$ & \\
$\begin{array}{c}\text { Halo } \\
\text { stars }\end{array}$ & $\mathrm{Pl}$ & 32500 & 1.30 & 5.0 & 10.0 & \\
& & & & & & \\
& Type & $\mathbf{N}$ & $\mathbf{M}_{\mathbf{C}}$ & $\mathbf{b}_{\mathbf{C}}$ & $\mathbf{r}_{\mathbf{C}}$ & \\
$\begin{array}{c}\text { Comp. } \\
\text { stars }\end{array}$ & $\mathrm{Pl}$ & 10000 & 0.40 & 0.195 & 3.0 & \\
\hline
\end{tabular}

the companion are set up with a cut-off mass $M_{j}$ and radial scalelength $b_{j}$ with $j=\mathrm{H}$ and $\mathrm{C}$, respectively, with the cut-off radius $r_{j}$.

The initially spherical halo, not being in virial equilibrium with the embedded disc, is allowed to relax in the potential of the dynamically frozen disc, whose mass is slowly turned on over time following the spline function

$$
u(t)=\left\{\begin{array}{ll}
\left(3-2 \frac{t}{t_{0}}\right) \cdot\left(\frac{t}{t_{0}}\right)^{2} & \text { for } t \leq t_{0} \\
1.0 & \text { for } t>t_{0}
\end{array},\right.
$$

where the turn-on time in our units has been chosen as $t_{0}=60$. The halo is relaxed for a total time period of $\Delta t=120$. From the resulting potential we then assign velocities to the disc particles, with the radial dispersion based on Toomre's stability criterion (Toomre 1964) and correcting for asymmetric drift.

The spherical companion galaxy is similar to the model referred to as csd in APB97. The mass $M_{\mathrm{C}}$ of the companion has been chosen such that the mass ratios of companion to the host disc and to total host mass $M_{\mathrm{tot}}=M_{\mathrm{D}}+M_{\mathrm{H}}$ are, respectively, $M_{\mathrm{C}} / M_{\mathrm{D}}=0.57$ and $M_{\mathrm{C}} / M_{\text {tot }}=0.20$. Based on the results of APB97, we expect to obtain with these mass ratios the formation of spokes and rings following the interaction. The initial model parameters are summarized in Table 5.1. The initial velocity distribution of the disc is shown in Fig. 5.1. The tangential velocity curve has its turn-over radius at roughly $2 a$ and stays flat in the outer disc. 
Table 5.2: Model and interaction description.

\begin{tabular}{lclll}
\hline Model & Name & $t_{\text {imp }}$ & Bar & $t_{\text {end }}$ \\
\hline Isolated model & I 0 & $\cdots$ & & $t=300$ \\
Central impact & $\mathrm{C} 1$ & $t=60$ & strong & $t=210$ \\
& $\mathrm{C} 2$ & $t=150$ & weak & $t=300$ \\
Major axis impact & $\mathrm{A} 1$ & $t=60$ & strong & $t=210$ \\
& $\mathrm{~A} 2$ & $t=150$ & weak & $t=300$ \\
Minor axis impact & $\mathrm{B} 1$ & $t=60$ & strong & $t=210$ \\
& $\mathrm{~B} 2$ & $t=150$ & weak & $t=300$ \\
\hline
\end{tabular}

\section{Units}

The adopted units for mass, distance, and time are $M=6 \times 10^{10} \mathrm{M}_{\odot}, \mathrm{R}=3 \mathrm{kpc}$ and $\tau=10^{7} \mathrm{yr}$, respectively, for which the gravitational constant $\mathrm{G}$ is unity. The dynamical time is $\tau_{\text {dyn }} \equiv\left(R_{\mathrm{h}}^{3} / \mathrm{G} M_{\mathrm{h}}\right)^{1 / 2}=4.8 \times 10^{7} \mathrm{yr}$, where $M_{\mathrm{h}}$ is the total mass within a sphere of radius equal to the half mass radius $r_{\mathrm{h}}$, which is, after relaxation of the halo, approximately $8.5 \mathrm{kpc}$ or, in terms of the disc scalelength, $2.8 \mathrm{a}$. The initial stellar disc rotation period in these units then corresponds to $t_{\mathrm{rot}} \equiv 2 \pi \tau_{\mathrm{dyn}} \approx$ $3 \times 10^{8} \mathrm{yr}$. A fixed gravitational softening length of $\epsilon=0.375 \mathrm{kpc}$ is used for all particles. An isothermal equation of state is used for the gas with a sound speed of $v_{\mathrm{s}}=12 \mathrm{~km} \mathrm{~s}^{-1}$. The corresponding thermal temperature of the gas is $10^{4} \mathrm{~K}$.

\section{Interaction parameters}

Before adding the companion to the model, we evolve the isolated barred host galaxy for $\Delta t=300$, or $3 \times 10^{9} \mathrm{yr}$. The initial centre of mass positions and velocities of the host and companion have then been obtained by integrating their orbits backward in time, starting from the impact time $t_{\text {imp }}$ at which the centre of mass of the companion lies in the disc plane. During this integration, the disc (with bar) and halo particles are frozen with respect to each other, but allowed to rotate as a single system with the (negative) angular frequency or pattern speed $\Omega_{\mathrm{p}}(t)$ of the bar, as determined from the isolated disc model (hereafter I0). The companion is represented by a point mass for this integration. This approximation is sufficient since the encounter is fast enough that dynamical friction does not significantly modify the orbit.

The impact velocity $v_{\text {imp }}$ of the companion has been chosen to be of the order of $4 v_{\text {esc }}$, with $v_{\text {esc }}$ being the escape velocity from the centre of the host system. The hyperbolic orbit is then integrated backward for a time period of $\Delta t=30$, which 
places the companion well outside the halo of the disc system. The simulations presented in this paper will be confined to almost perpendicular passages. Impacts along trajectories 30 degrees (and sometimes higher) from the disc normal still yield ring shaped features (Lynds \& Toomre 1976). The effects of oblique impacts have been studied by Toomre (1978), Athanassoula (1999) and APB97. An overview of the isolated and interaction models is given in Table 5.2. The columns, from left to right, give the type and the name of the model, the impact time $t_{\text {imp }}$, the strength of the bar at $t_{\mathrm{imp}}$ and the end time $t_{\text {end }}$ of each simulation.

\subsection{Results}

\subsubsection{Model I 0 : isolated barred galaxy}

The morphological evolution of the stellar and gaseous disc is shown in Fig. 5.2. The model of the disc galaxy was constructed so as to be globally unstable to nonaxisymmetric perturbations and form a large-scale bar in a few disc rotations. At $t=60$, or after some $2 t_{\text {rot }}$, the stellar bar reaches its maximum strength, defined by the normalized amplitude of the $m=2$ Fourier component of the stellar disc particle distribution inside a cylindrical radius of $3.75 \mathrm{kpc}$ and one scaleheight of the disc plane. Care was taken in all models not to include any power from spiral features. The result is shown in Fig. 5.3(a). At the time of its maximum strength the bar has a major axis length of $a=6 \mathrm{kpc}$ and an axial ratio of approximately $3: 1$, as determined by simple measurements of the stellar isodensity contours. Both stellar and gaseous trailing spiral arms emerge from the end of the bar. While the stellar arms slowly dissolve and are hardly visible by $t=100$, large spiral features in the gas persist throughout the run. The spiral pattern in the gas evolves from a two-arm to a flocculent-type spiral after the stellar arms have dissolved. The gas also forms straight off-set shocks at the leading edge of the stellar bar. The shape and position of the shock loci indicate that the Lagrangian radius $r_{\mathrm{L}}$ is small and that the resonant $x_{2}$ orbit family is either absent or negligible at this early stage of evolution (Athanassoula 1992b).

As soon as the bar forms, a substantial gas inflow sets in owing to its gravitational torque, and within some $40 \tau$, or approximately two bar rotations, some 50 per cent of the total gas, or $4.8 \times 10^{9} \mathrm{M}_{\odot}$, is driven towards the centre (Fig. $5.3 \mathrm{c}$ ) and accumulates there in a dense oval nuclear disc. This disc is elongated along the bar's major axis and has a mean radius of approximately $r=0.36 \mathrm{kpc}$. Although the nuclear disc appears to be aligned with the bar's major axis, its shape and orientation could well be affected by the numerical softening, since its radius is a little less than half the softening length $\epsilon$. The mass of the nuclear disc represents some 18 per cent of the total dynamical mass within a radius of $1.0 \mathrm{kpc}$. As a result of the growing mass concentration at the centre, the amplitude of the stellar bar decreases rapidly (Heller $\&$ Shlosman 1994). At $t=80$ the initial burst of inflow slows down 

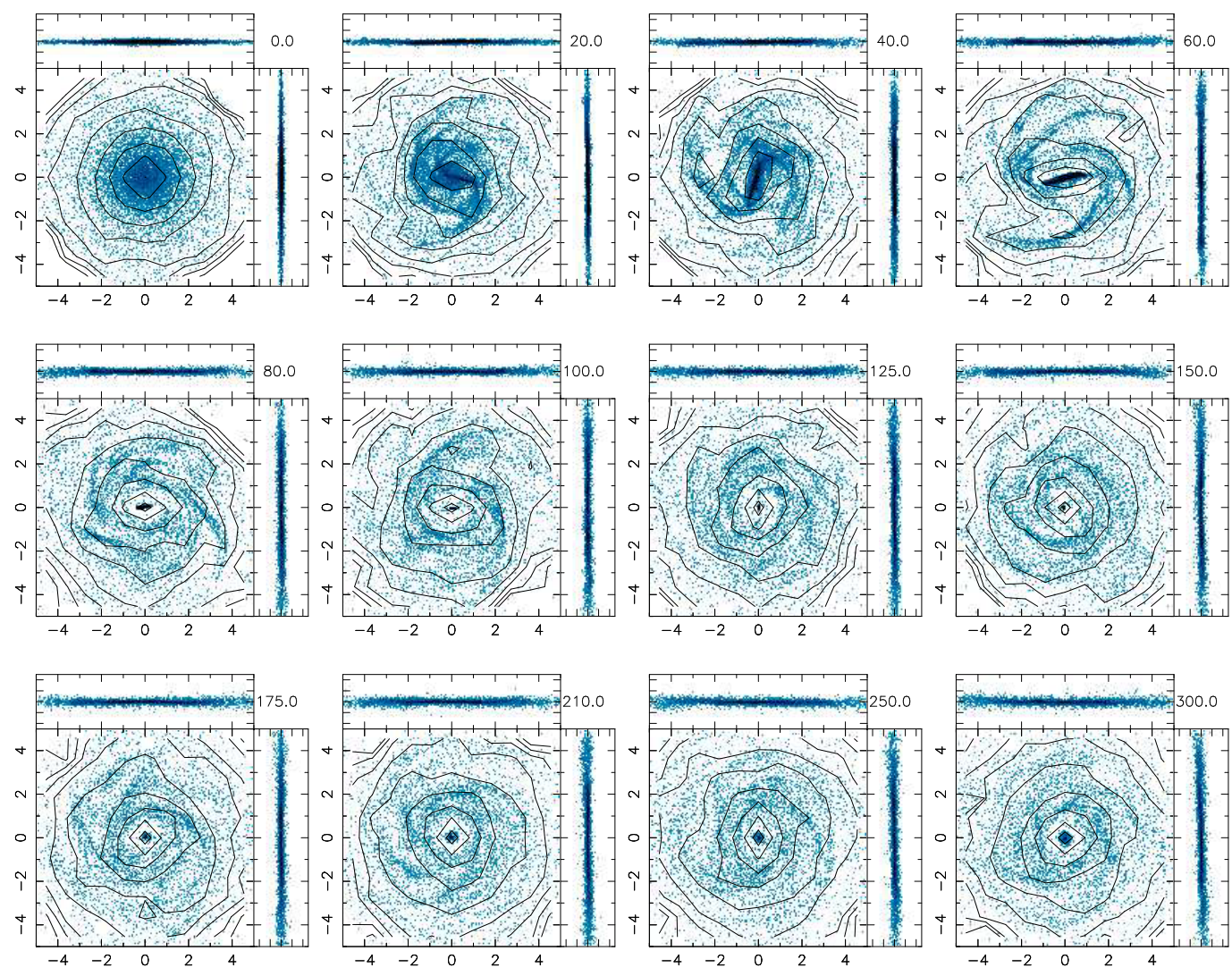

Figure 5.2: Evolution of the stellar and gaseous disc in the isolated barred galaxy model I0. We show the face-on grey-scale density plot and the edge-on particle distribution of the gas. The stellar isodensity contours are shown face-on. The disc rotation is counter-clockwise. The time is given in the upper right-hand corner of each panel in model units.

and the stellar bar becomes quasi-stable (see Fig. 5.3 a), decaying at a much slower rate of $3 \times 10^{-4} \tau^{-1}$, as estimated by a linear least-squares fit. Likewise, owing to the inflow of gas to the centre, the pattern speed $\Omega_{\mathrm{p}}$ of the bar, as given by the phase angle of the $m=2$ Fourier component, increases linearly (Heller \& Shlosman 1994) till $t=80$ (Fig. $5.3 \mathrm{~b}$ ). After the central inflow of gas stops, i.e. at around $t \approx 90$, the bar is rotating uniformly with a constant rate of $\Omega_{\mathrm{p}}=0.3 \tau^{-1}$, or $29 \mathrm{~km} \mathrm{~s}^{-1} \mathrm{kpc}^{-1}$, throughout the rest of the simulation.

The gaseous nuclear disc (hereafter n-disc) that has formed by the burst of gas inflow at first remains connected with the outer disc by two trailing spiral segments, which by way of their shocks feed material inward and thus contribute to its growth. Initially the nuclear disc is oval in shape, but as the bar weakens it becomes more 


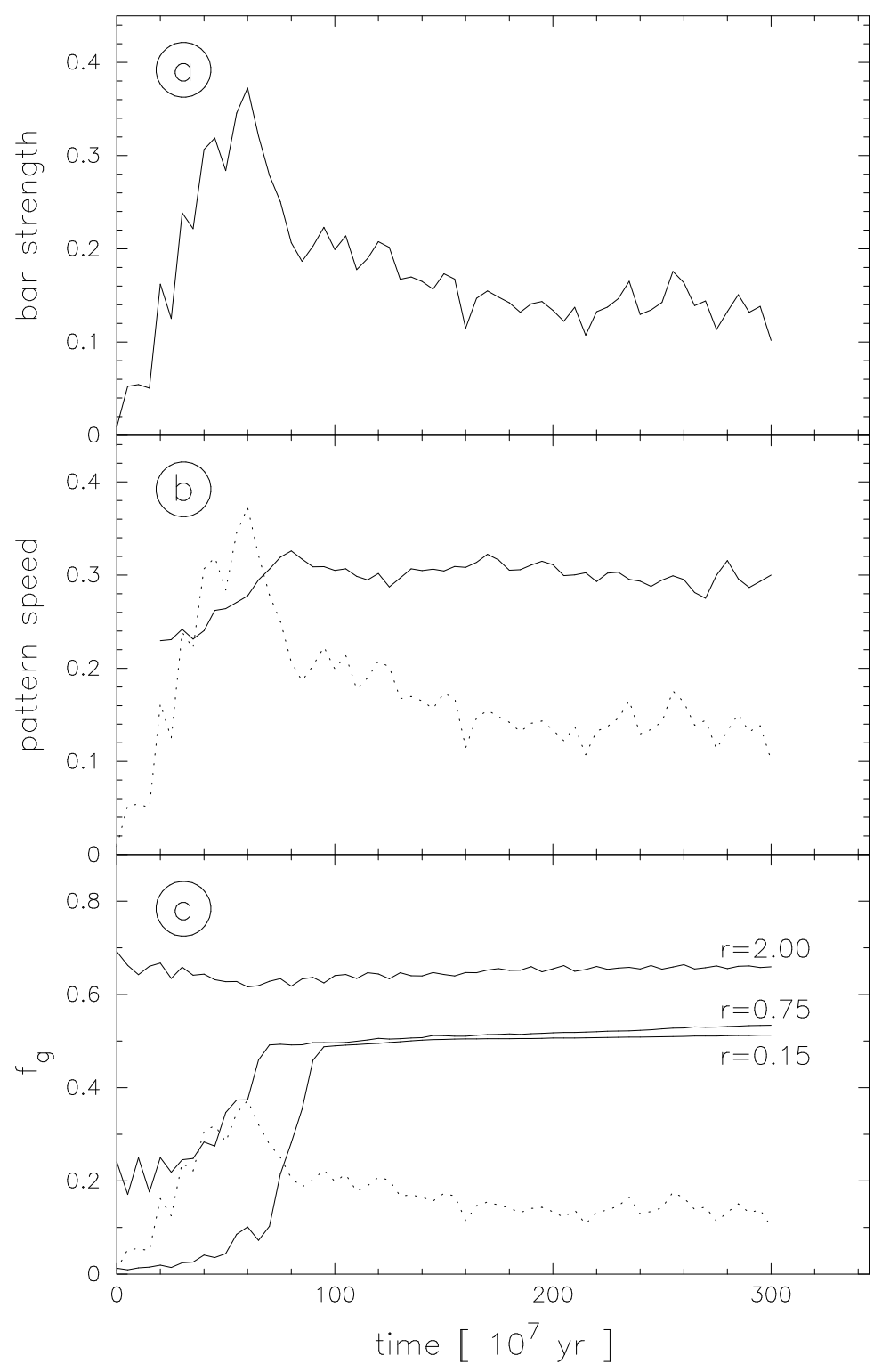

Figure 5.3: The time-evolution of (a) the bar strength, (b) the pattern speed and (c) the gaseous mass fraction $\left(f_{\mathrm{g}}\right)$ within a constant radius in the isolated model I 0 (full lines). In panels (b) and (c) the bar strength is plotted with a dotted line for better comparison.

circular. After $t=95$ the nuclear disc does not grow in mass anymore and the still inflowing gas starts to accumulate in a circumnuclear disc (hereafter cn-disc) surrounding the nuclear disc. This elongated cn-disc is orientated perpendicular to the bar's major axis and has a lower surface density than the nuclear disc. In addition the 

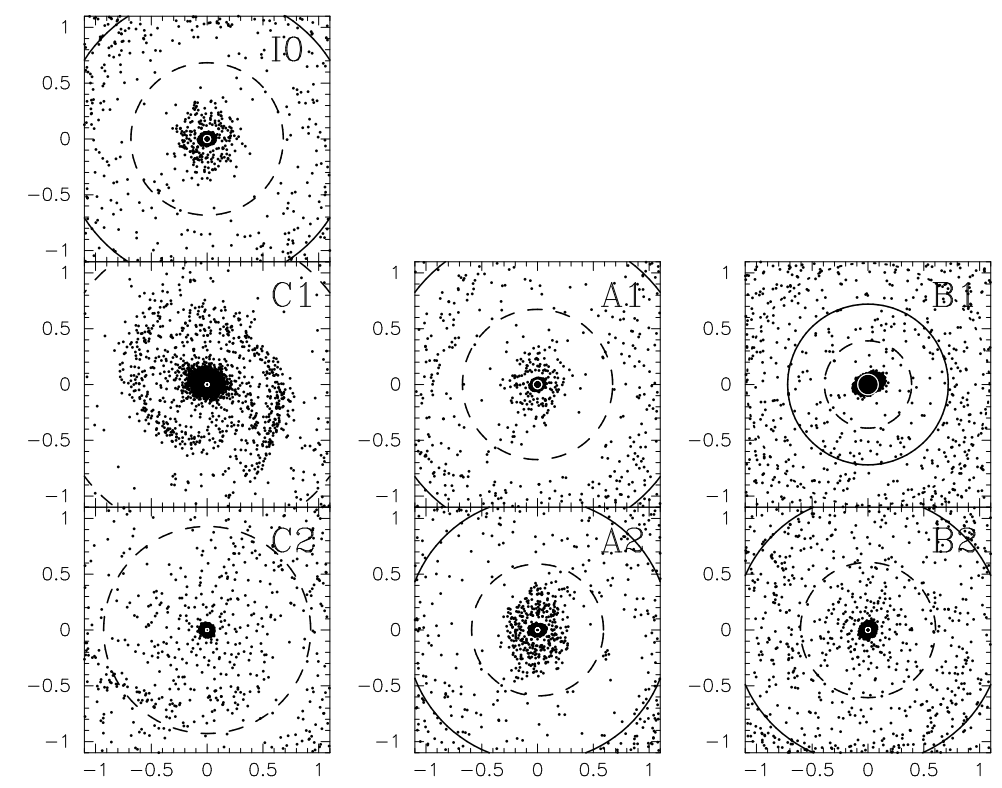

Figure 5.4: Face-on plot of the gas particle distribution in the central disc region. The panels show the final gaseous mass distribution at the end of the different simulations (see Table 2), identified in the upper right-hand corner of each panel. The circles mark the radius of the resonances obtained from the linear analysis: oILR (dashed lines) and UHR (full lines).

cn-disc is more extended in size than the n-disc and has a radius of $r_{\text {cnd }}=0.9 \mathrm{kpc}$ at the end of the simulation. The final morphology of the nuclear region is shown in Fig. 5.4. The orientation of both the nuclear and the circumnuclear disc relative to the stellar bar indicate that the gas populates the main families of periodic orbits in the barred potential (see, for example, Contopoulos \& Papayannopoulos 1980; Athanassoula et al. 1983). The $x_{1}$ orbits, which support the bar, are elongated along the bar's major axis, while the $x_{2}$ orbits between the inner and outer Inner Lindblad Resonances (hereafter: iILR and oILR, respectively) are orientated perpendicular to the bar (Contopoulos \& Mertzanides 1977).

In order to identify the presence and location of the main planar resonances in the disc at different times we average the mass distribution azimuthally and derive the $\Omega_{\mathrm{c}}-\kappa / 2$ curve, where $\Omega_{\mathrm{c}}$ and $\kappa$ denote the circular and the epicyclic frequency, respectively. At the end of the run $(t=300)$ this curve shows a pronounced maximum at a radius of $r=0.5 \mathrm{kpc}$ exceeding the pattern speed $\Omega_{\mathrm{p}}$ of the bar, indicating the presence of ILRs. The resonant radii, obtained with this approximation, are given in Table 5.3.

At its maximum strength, however, the stellar bar provides a strong non-linear perturbation to the gravitational potential of the disc and applying linear theory 
Table 5.3: Resonances in the disc at different model times.

\begin{tabular}{rccccccc}
\hline Time & Model & $\Omega_{\mathrm{p}}^{\text {end }}$ & i ILR & o ILR & UHR & CR & OLR \\
\hline 60 & I0 & 0.28 & 0.11 & 0.23 & 1.40 & 2.16 & 3.85 \\
150 & I0 & 0.30 & 0.03 & 0.64 & 1.25 & 1.99 & 3.48 \\
210 & I0 & 0.30 & 0.03 & 0.64 & 1.27 & 2.01 & 3.52 \\
& C1 & 0.11 & 0.02 & 1.37 & 2.26 & 3.52 & 4.78 \\
& A1 & 0.28 & 0.04 & 0.67 & 1.38 & 2.12 & 3.71 \\
& B1 & 0.53 & 0.09 & 0.39 & 0.72 & 1.09 & 1.90 \\
300 & I0 & 0.29 & 0.03 & 0.68 & 1.31 & 2.08 & 3.68 \\
& C2 & 0.16 & 0.01 & 0.93 & 2.22 & 3.63 & 4.93 \\
& A2 & 0.28 & 0.02 & 0.59 & 1.18 & 1.93 & 3.47 \\
& B2 & 0.33 & 0.02 & 0.61 & 1.20 & 1.85 & 3.14 \\
\hline
\end{tabular}

to identify the dynamical resonances is not sufficient. A more reliable way is to search for the existence of the main orbit families (Athanassoula 1992a). For this we constructed surfaces of sections (SOS) by integrating orbits of a given Jacobian energy $\mathrm{E}_{\mathrm{J}}$ in the equatorial plane, marking the points in the $(y, \dot{y})$ plane each time the orbits cross the line $x=0$ with $\dot{x}<0$ (see, for example, Binney \& Tremaine 1987). The gravitational potential of each snapshot has been calculated on a nonequally spaced Cartesian grid with a size of 6 by $6 \mathrm{kpc}$. From that, the potential and its derivatives are evaluated by using a piecewise polynomial function represented by a tensor product of one-dimensional B-splines. The times chosen for the SOS, i.e. at impact time and at the end of each run, are given in Table 5.2. We were thus able to confirm the presence of the ILRs in all of the cases, except for models I 0 $(t=60)$ and $\mathrm{C} 1(t=210)$.

Most of the gas initially residing within corotation is driven towards the centre, resulting in a noticeable deficiency of gas outside the cn-disc, which could be interpreted as representing an $\mathrm{H}$ i hole. In contrast to this hole, an oval ring of gas accumulates near the end of the weak stellar bar, close to the position of the ultraharmonic resonance (UHR) at $r=2.25 \mathrm{kpc}$. After the resonance ring has formed $(t=175)$ little further net radial redistribution of the gas (less than 1 per cent) occurs.

The stellar disc thickens vertically. We follow the time-evolution of its radially

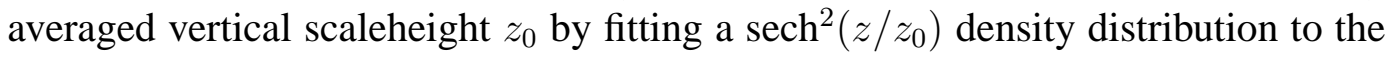
particle distribution. The result is shown in Figure 5.5. We find a linear increase in the vertical scaleheight by a total of roughly 25 per cent in the isolated model. We also notice a thickening of the gaseous disc, especially some slight flaring in the outer parts. Some of the thickening, however, might be induced numerically, either owing to the limited number of particles in the model or to the relatively 




Figure 5.5: Time-evolution of the radially averaged scaleheight $z_{0}$ of the stellar disc. The full line in both panels shows the isolated model I0. In panel (a) the evolution of the models $\mathrm{C} 1, \mathrm{~A} 1$ and B1 with an early impact is plotted with dotted, dashed, and dash-dotted lines, respectively. The same for panel (b) for the late impact models C2, A2 and B2. The impact times are marked by an arrow in both panels.

large softening. Since we use the same model later for the host galaxy, it will show the same numerical heating and any further change in scaleheight may be attributed to the interaction.

In this isolated model there is no perceptible sign of a peanut-shaped bar due to a buckling instability. This could be due to the fact that, even if the stellar disc were susceptible to such an instability, the presence of the gas would greatly dampen it (Berentzen et al. 1998).

\subsubsection{Interacting models}

The two times chosen for the impact of the companion correspond to the time (1) when the bar is at its maximum strength $\left(t_{\mathrm{imp}}=60\right)$ and $(2)$ when the bar is weak 

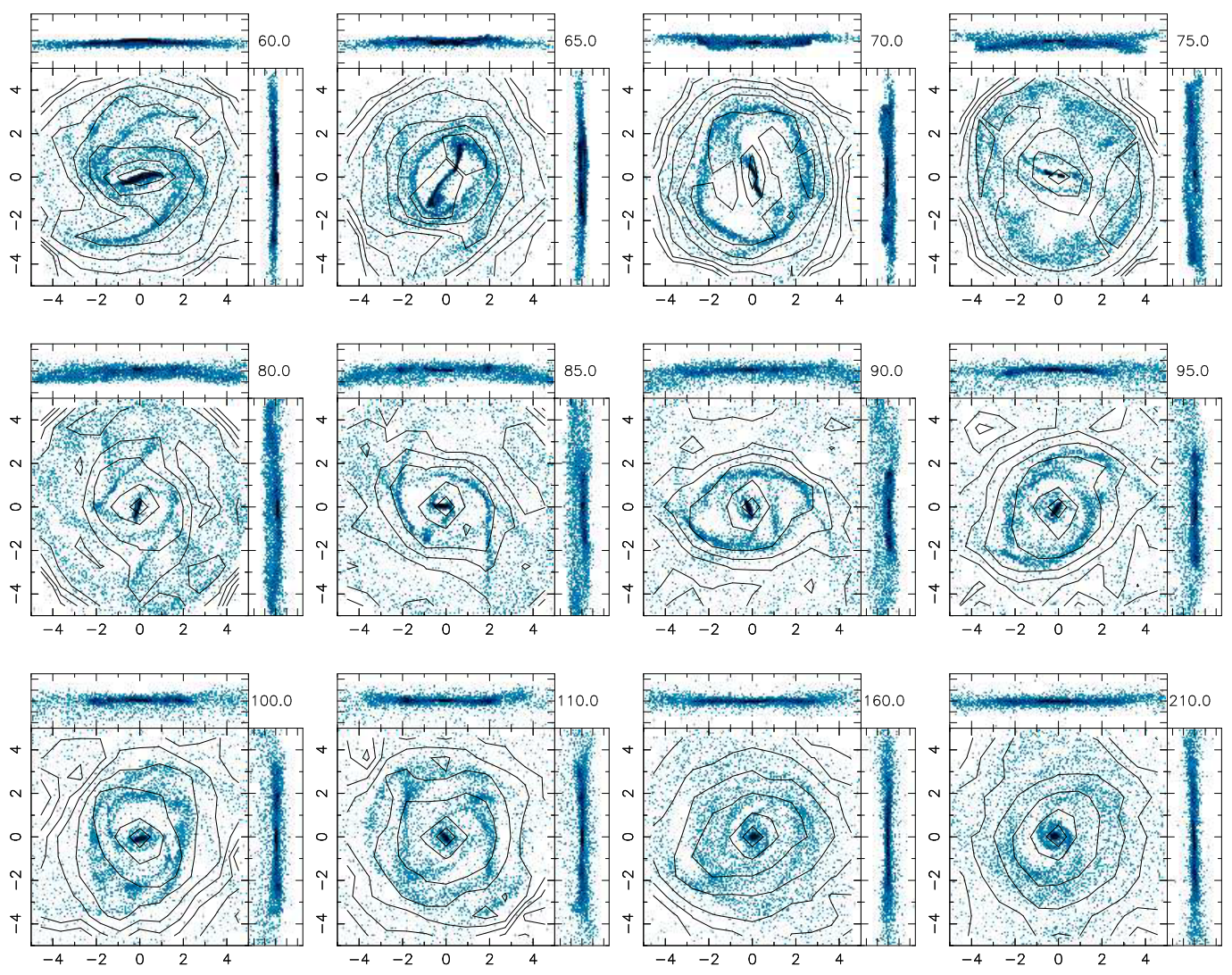

Figure 5.6: Evolution of the gaseous and stellar disc in the early central passage model C1. The layout is as for Fig. 5.2.

and has settled down to a quasi-stable state $\left(t_{\mathrm{imp}}=150\right)$. Both times represent a characteristic epoch in the evolution of the disc. In the first case the bar has already formed and dynamically dominates the disc, but has still not had time to modify significantly the radial mass distribution of stars and gas. In the second case the stellar bar has weakened and the dynamical influence of the bar can be neglected compared to the effects of the interaction alone. The impact position, i.e. the position of the centre of mass of the companion in the equatorial $z=0$ plane of the disc at $t_{\text {imp }}$, has also been varied with respect to the bar. In the models with major axis impacts, the impact position has a distance from the centre of $r_{\mathrm{imp}}=6.0 \mathrm{kpc}$, which corresponds roughly to the corotation radius in the isolated model. For the minor axis impacts, the distance is $r_{\mathrm{imp}}=3.0 \mathrm{kpc}$. 

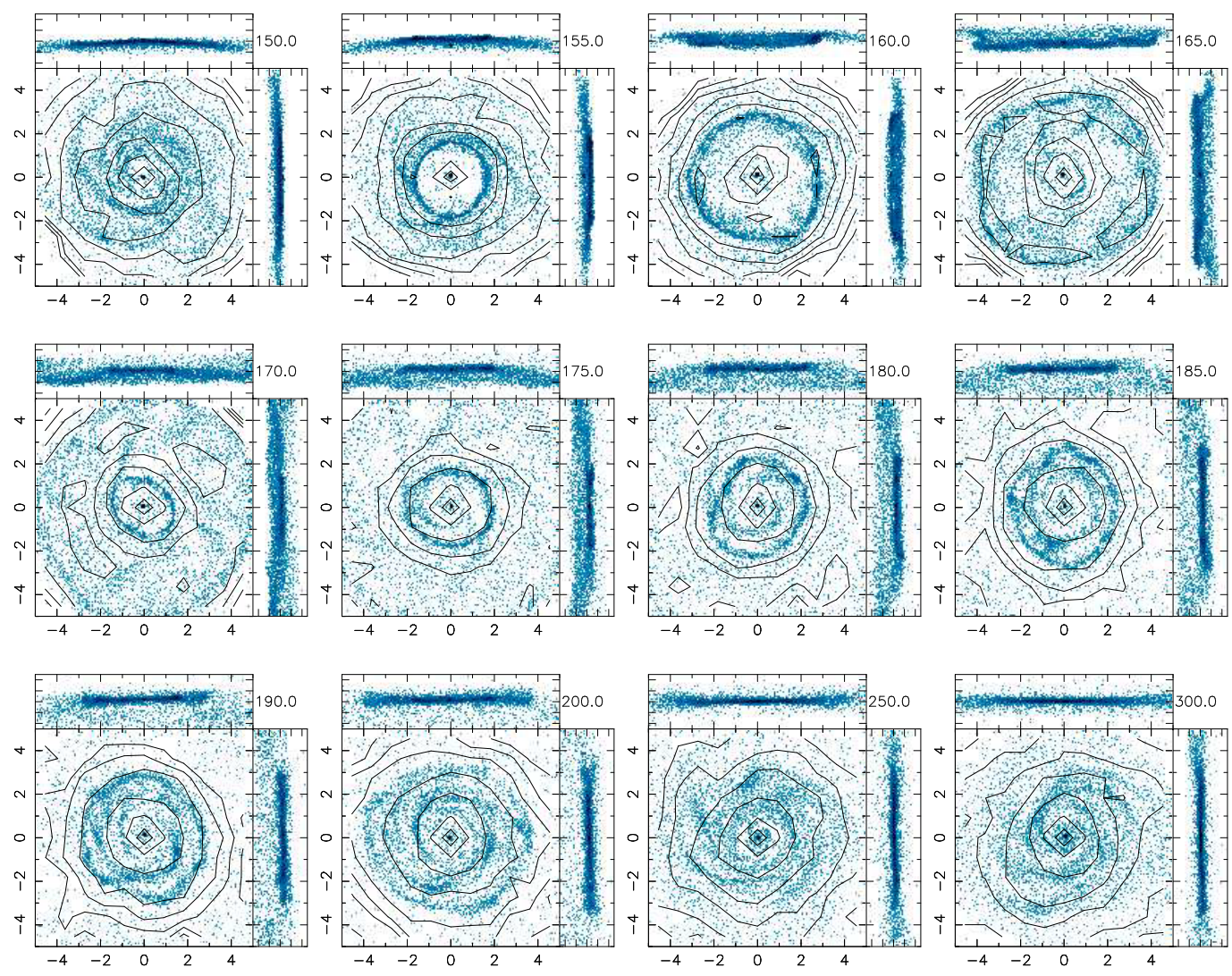

Figure 5.7: Same as Fig. 5.6, but for the late central passage model C2.

\subsubsection{Central passage : models $C 1$ and $C 2$}

In the simulations described in this section the companion hits the disc of the host galaxy at its centre. The morphological evolution of the models $C 1\left(t_{\mathrm{imp}}=60\right)$ and $\mathrm{C} 2\left(t_{\mathrm{imp}}=150\right)$ is shown in Figs 5.6 and 5.7, respectively. Prior to impact, both models show an axisymmetric vertical bending in both the stellar and the gaseous disc, with the inner regions pulled out in the direction of the approaching companion, as illustrated for the gaseous disc in the figures at $t=60$ and $t=150$ for models $\mathrm{C} 1$ and $\mathrm{C} 2$, respectively.

The impact of the companion produces some expanding rings in the stars and gas, of which the latter are more sharply pronounced. The axial symmetry of the disc in model $\mathrm{C} 1$ is broken by the strong stellar bar and therefore the induced rings do not maintain their circular shape at larger radii (Fig. 5.6, after $t=70$ ). Between the first and the second ring form several spokes, which are more pronounced in the gas, while their stellar counterparts are hardly visible by eye. All these induced 

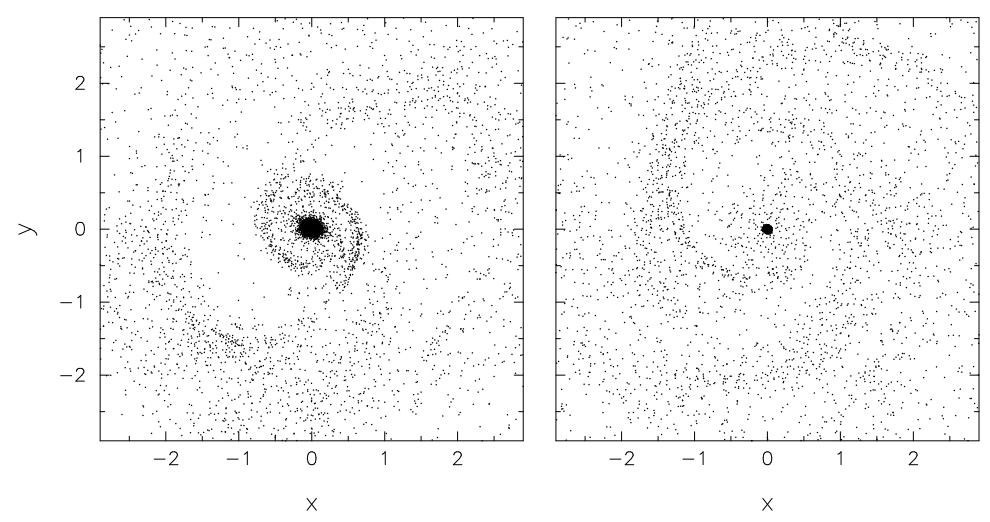

Figure 5.8: Face-on plot of the final gas particle distribution in the inner disc region of models C1 (left-hand panel) and C2 (right-hand panel) at the end of the simulation.
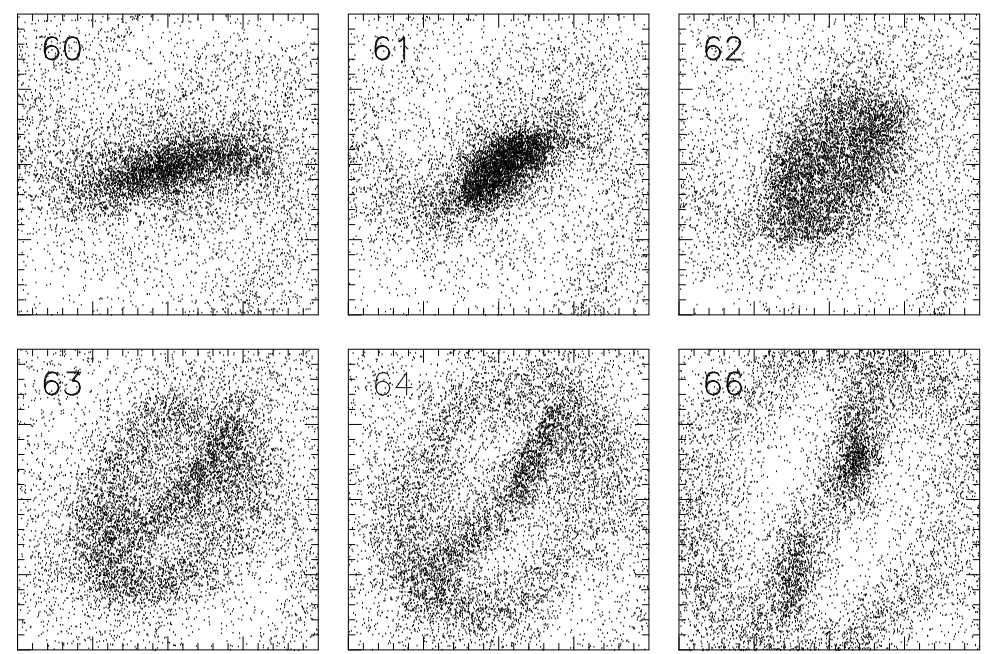

Figure 5.9: Evolution of the stellar bar in model $\mathrm{C} 1$ after the impact. We show the face-on particle distribution of the stellar disc. The corresponding times in model units are given in the upper left-hand corner of each frame and the size of the box corresponds to $12 \mathrm{kpc}$.

features persist for only a few dynamical times after which the disc settles down once again to a quasi-steady state. Although the stellar bar gets destroyed in both simulations, its dynamical imprint remains in the gas: namely the nuclear and circumnuclear discs, and the inner pseudo-ring. These components become visible again shortly following the impact and survive in the disc till the end of the runs (see Fig. 5.8). 

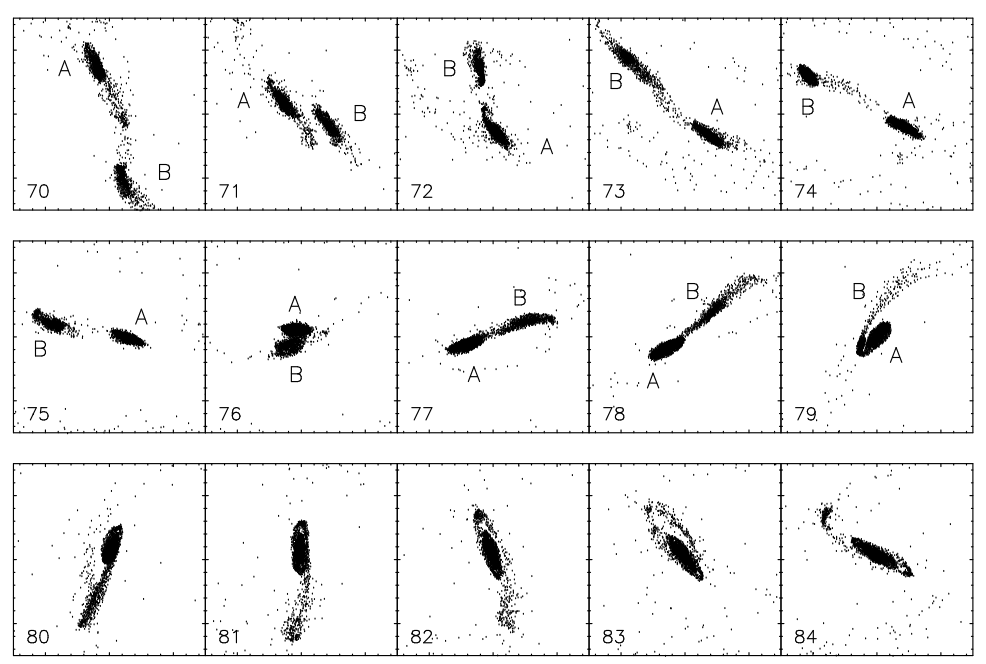

Figure 5.10: Evolution of the gaseous disc in model $\mathrm{C} 1$ after the impact. We show the face-on gas particle distribution. The size of one box corresponds to $4.5 \mathrm{kpc}$. The two gaseous fragments are labelled A and B and the time in model units is given in the lower left-hand corner of each panel.

\section{Evolution of the bar}

In the early-impact model $\mathrm{C} 1$, both the stellar bar and the gas, which had accumulated prior to the impact in the shock loci inside the bar, get torn apart by the impact into two separate fragments. Fig. 5.9 shows this evolution for the stellar bar. When the companion passes through the disc, it exerts an extra inwards gravitational force on the disc particles and causes their orbits to contract. This affects the main orbit families of the bar and therefore the bar temporarily shortens somewhat (Fig. 5.9, $t=61$ ). After the companion has left the disc there is a strong rebound of the orbits, resulting in a radially expanding density wave. With the expansion of the particle orbits, the bar gets torn apart and the extremities of the two stellar fragments remain temporarily connected with the expanding stellar ring. At all times the two bar fragments are enclosed by the expanding ring. When the ring reaches a radius of approximately $r=6 \mathrm{kpc}$, the two bar fragments detach from it and sink back towards the centre, where they finally merge some $\Delta t=10$, or $0.1 \mathrm{Gyr}$, after the impact and form a dense, almost axisymmetric centre. Likewise, the gas concentrations at the shock loci inside the bar get torn apart, following the movement of the stellar bar fragments. As shown in Fig. 5.10 the two separated gas fragments flow back and forth inside the bar potential being trapped around $x_{1}$-like orbits. During this period they appear as two separate nuclei, before they finally merge and form a single nuclear disc some $0.2 \mathrm{Gyr}$ after the impact.

In the later impact model $\mathrm{C} 2$, the stellar bar already has been weakened signif- 
icantly owing to the gas inflow. In this case also the impact produces expanding rings in the disc, but no stellar or gaseous fragments as in model $\mathrm{C} 1$.

In both models the stellar bar gets destroyed by the interaction almost immediately after the impact. The bar strength as a function of time is shown in Fig. 5.19 (full line). In model C2 the already weak bar gets destroyed when the first stellar ring detaches from the central region. The temporary increase in the $m=2$ amplitude after the impact in model C1 results from the two stellar fragments which contribute to a bisymmetric distribution, but does not represent a stronger bar. No further stellar signatures of the former bar are left in the disc at the end of the runs.

\section{Rings and spokes}

The passage of the companion through the disc excites both radial and vertical oscillations in the disc. The induced radial oscillatory motion of the particles produces expanding density ring-waves (see, for example, Lynds \& Toomre 1976) centred on the impact position, while the vertical oscillations lead to an increase of the vertical velocity dispersion and a significant thickening of the disc.

As shown in Figs 5.6 and 5.7, an expanding ring is produced by the impact in both the stars and the gas. The ring first becomes visible in the gas, since the stellar ring is much broader and only becomes visible at sufficiently large radii. As the ring expands outward it becomes broader and its amplitude in both stars and gas decreases slowly as predicted by the impulse approximation (see, for example, Binney \& Tremaine 1987). The ring in model C1 becomes asymmetric (Fig 5.6, $t=70$ ), since the underlying disc potential is perturbed by the presence of the stellar bar at least in the early phases of ring formation. In model $\mathrm{C} 2$, however, the induced ring is more symmetric, since the perturbation due to the bar is weaker. In both models, the first ring reaches about $9 \mathrm{kpc}$, at which point the inner part of the gaseous ring starts to fragment, with most of its mass flowing back towards the inner few kpc.

Following the first, a second expanding ring forms in both models, and becomes first visible in the gas after some $\Delta t=20$, or $0.2 \mathrm{Gyr}$ after the impact (see Fig. 5.11). The second ring expands out to a radius of $r \approx 6 \mathrm{kpc}$ where it dissolves and its material is redistributed in the central region. In the stellar disc the second ring is hardly visible, since its amplitude is too low and it does not expand as far out as the first one. While only two rings form in the stars - a third one can (hardly) be identified only in the radial density profile of the disc - we find several consecutive expanding ring features in the gas that are more asymmetric than the first two rings (e.g., Fig. $5.7 t=190,200$ and Fig. $5.12 t=57$ ).

The gas fragments that detach from the inner side of the first ring flow back to the central disc region and are sheared out by the differential rotation of the disc, forming several spokes between the two collisional rings (Fig. 5.11). As noted by APB97, spokes form only between the first and the second ring, the presence of the latter being necessary. The properties of spokes in collisional ring galaxies are 

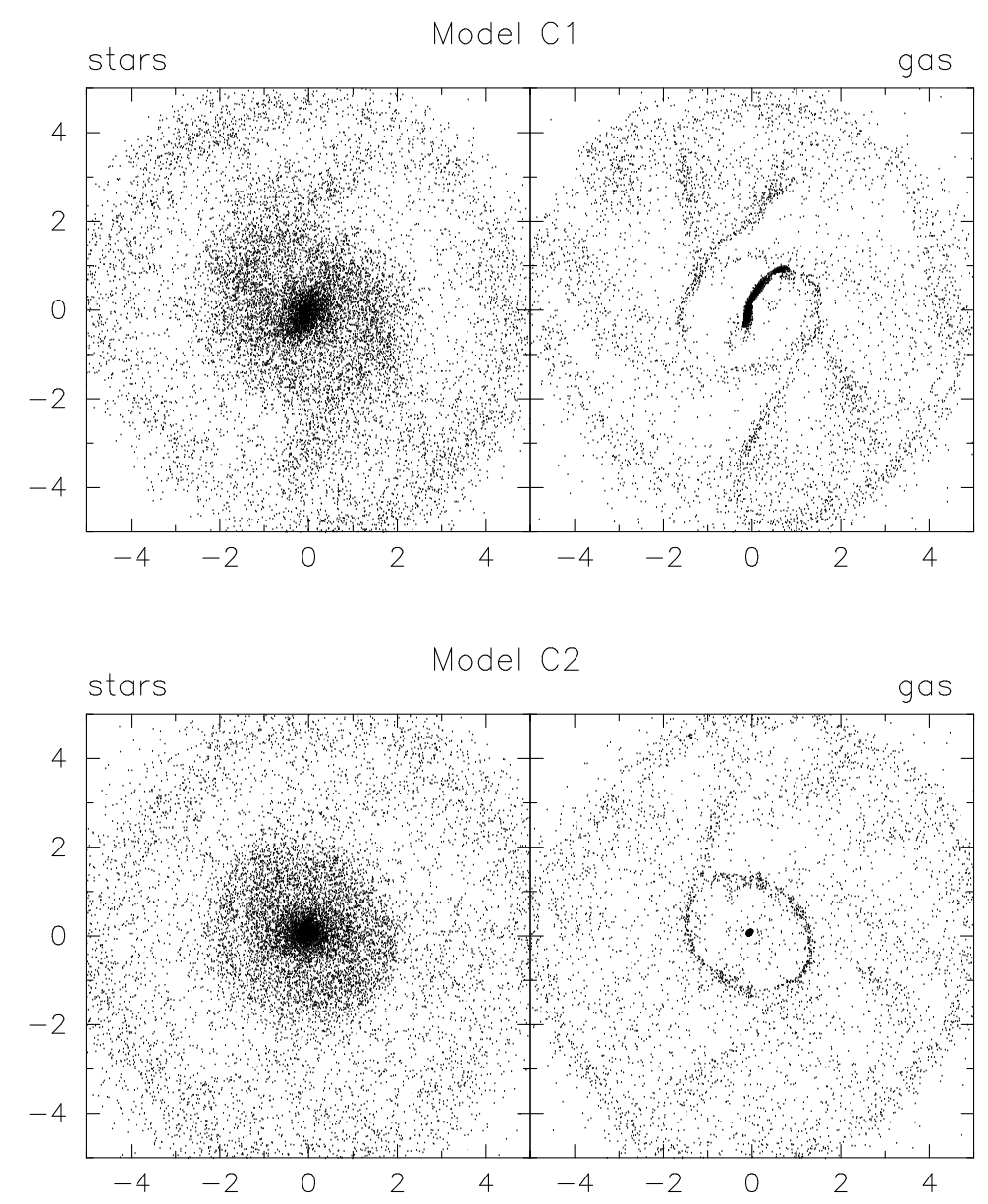

Figure 5.11: Face-on particle distribution of models $\mathrm{C} 1$ and $\mathrm{C} 2$ at time $\Delta t=20$ after the impact. The left- and right-hand frames show the stellar and gaseous disc, respectively. Only half the stellar particles are plotted for clarity. The spokes form between the first and second ring, the latter being visible mainly in the gas at this time. While the gaseous spokes are very prominent their stellar counterparts are less or hardly visible.

discussed in detail by Hernquist \& Weil (1993).

To track the position of the rings and derive their expansion velocity, we determine the azimuthally averaged density of annuli of varying radii in the disc. Then we fit a polynomial function to subtract the background of the disc and a Gaussian to fit the density distribution inside the ring. Using this method we get information about the position, width and mass of the rings. The results are shown in Fig. 5.13. We find that the gaseous ring traces the position of the stellar ring, i.e. its position coincides with that of the stellar ring (Fig. 5.13a) and both expand with the same velocity. With increasing radius the stellar and gaseous rings become broader 

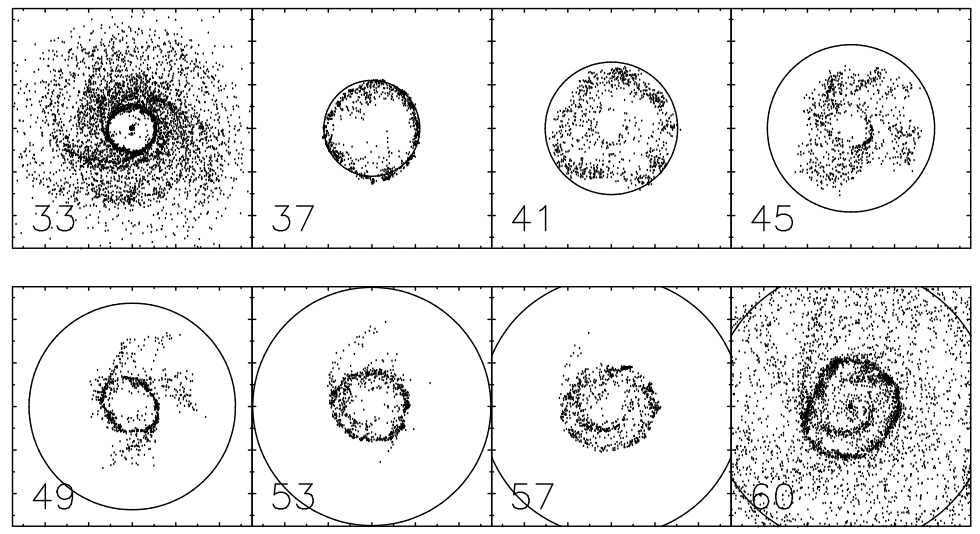

Figure 5.12: Expansion of the gaseous rings in model $\mathrm{C} 2$. The circle in the panels shows the position of the first ring. The time in model units is given in the lower left-hand corner of each panel. The first and the last panel show the SPH particle distribution of the whole disc, while the remaining panels include only the particles contributing to the first gaseous ring at $t=33$.

(Fig. 5.13b) and less dense, as predicted by the impulse approximation (see, for example, Binney \& Tremaine 1987).

By integrating the Gaussian with the fitted parameters we can estimate the gas mass contributing to the rings. Fig. 5.13c shows the mass of the gaseous rings as a function of time. Unlike stars, the orbits of the gas cannot cross and up to $t=40$ gas is piled up in the ring as it expands outward. Owing to shock dissipation and self-gravity of the ring, its inner parts start to fragment and thus its mass decreases. We fit a logarithmic function to the radii of the gaseous rings from which we derive their expansion velocity. Fig. 5.14 shows the position and expansion velocity of the first and second ring in model $\mathrm{C} 2$. As has been previously found (see, for example, APB97) the expansion velocity of the rings decreases with radius, as predicted by the impulse approximation. The expansion velocity of the first ring drops gradually from $88 \mathrm{~km} \mathrm{~s}^{-1}\left(7.5 v_{\mathrm{s}}\right)$ to $35 \mathrm{~km} \mathrm{~s}^{-1}\left(3 v_{\mathrm{s}}\right.$, where $v_{\mathrm{s}}$ is the sound speed in the gas). The second ring starts with a lower velocity, which decreases from $29 \mathrm{~km} \mathrm{~s}^{-1}$ $\left(2.5 v_{\mathrm{s}}\right)$ to $11.72 \mathrm{~km} \mathrm{~s}^{-1}\left(1 v_{\mathrm{s}}\right)$.

In Figs $5.15 \mathrm{a}$ and $\mathrm{b}$ we show the isocontours of the radial velocities for the stellar and gaseous disc, respectively, as a function of both radius and time. It shows the radial oscillation of the disc material, first inward following the impact, followed by an outward movement as the ring passes through, and finally again inward. The amplitude of this motion can be seen to be considerable. With each passage of a ring the local radial velocity dispersion (not shown here) in the disc increases, until further density enhancements of this kind are no longer supported.

The passage of the companion also gives rise to vertical oscillations in the disc 


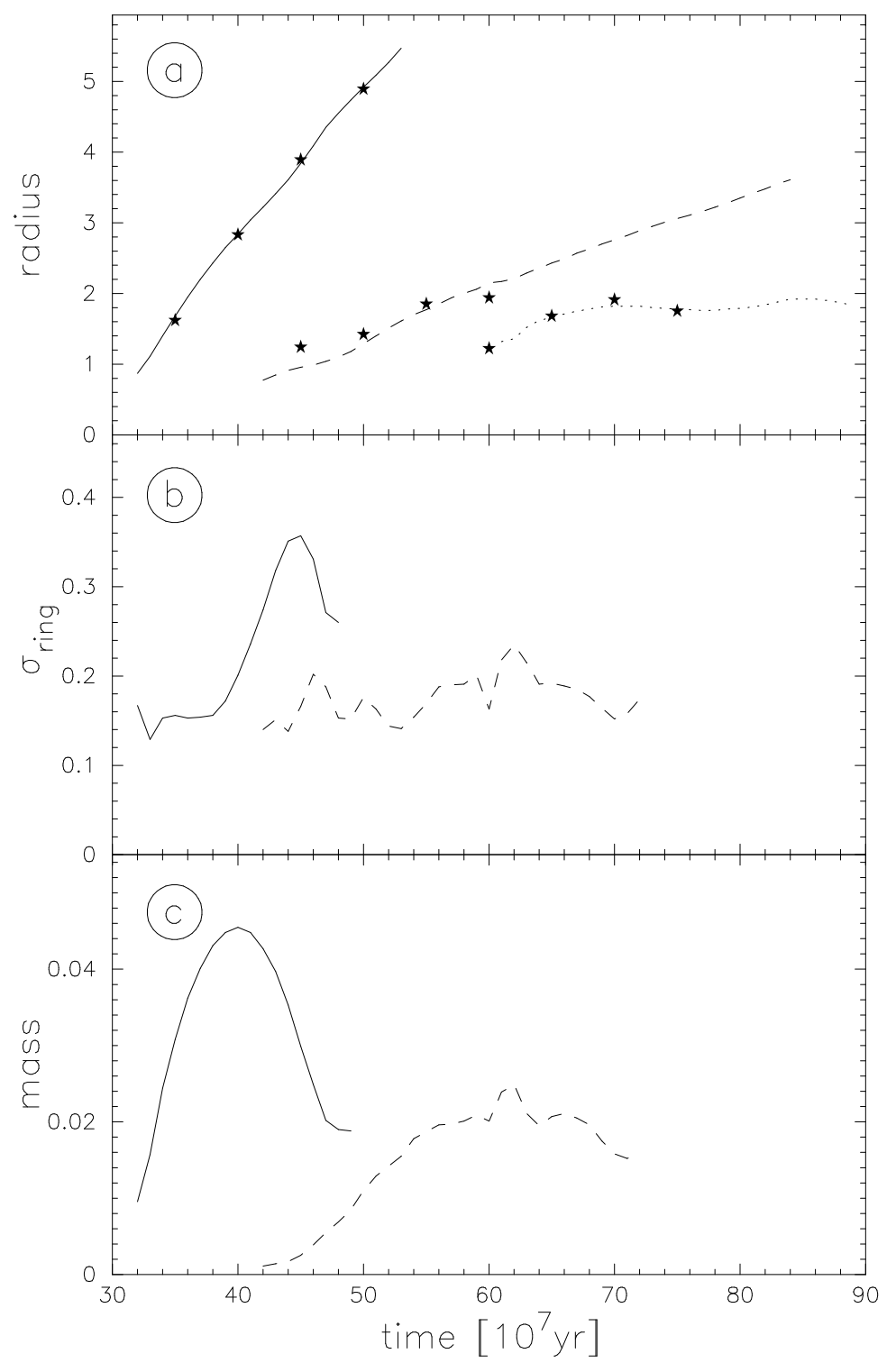

Figure 5.13: Properties of the stellar and gaseous rings in model C2. Panel (a) shows the mean radius of the three gaseous (full, dashed and dotted lines, respectively) and stellar rings (marked by stars) as a function of time. Panel (b) shows the width $\sigma$ of the gaseous rings obtained from a Gaussian fit. Panel (c) shows the mass of these rings.

(see, for example, Weinberg 1991; Mihos \& Hernquist 1994) giving it a layered appearance in the edge-on projection (see Fig. 5.6, e.g., $t=75$ and Fig 5.7, e.g., $t=165)$. The same type of vertical motion can be seen in the numerical models by Lynds \& Toomre (1976) and also in Mihos \& Hernquist (1994). In fact the layered 

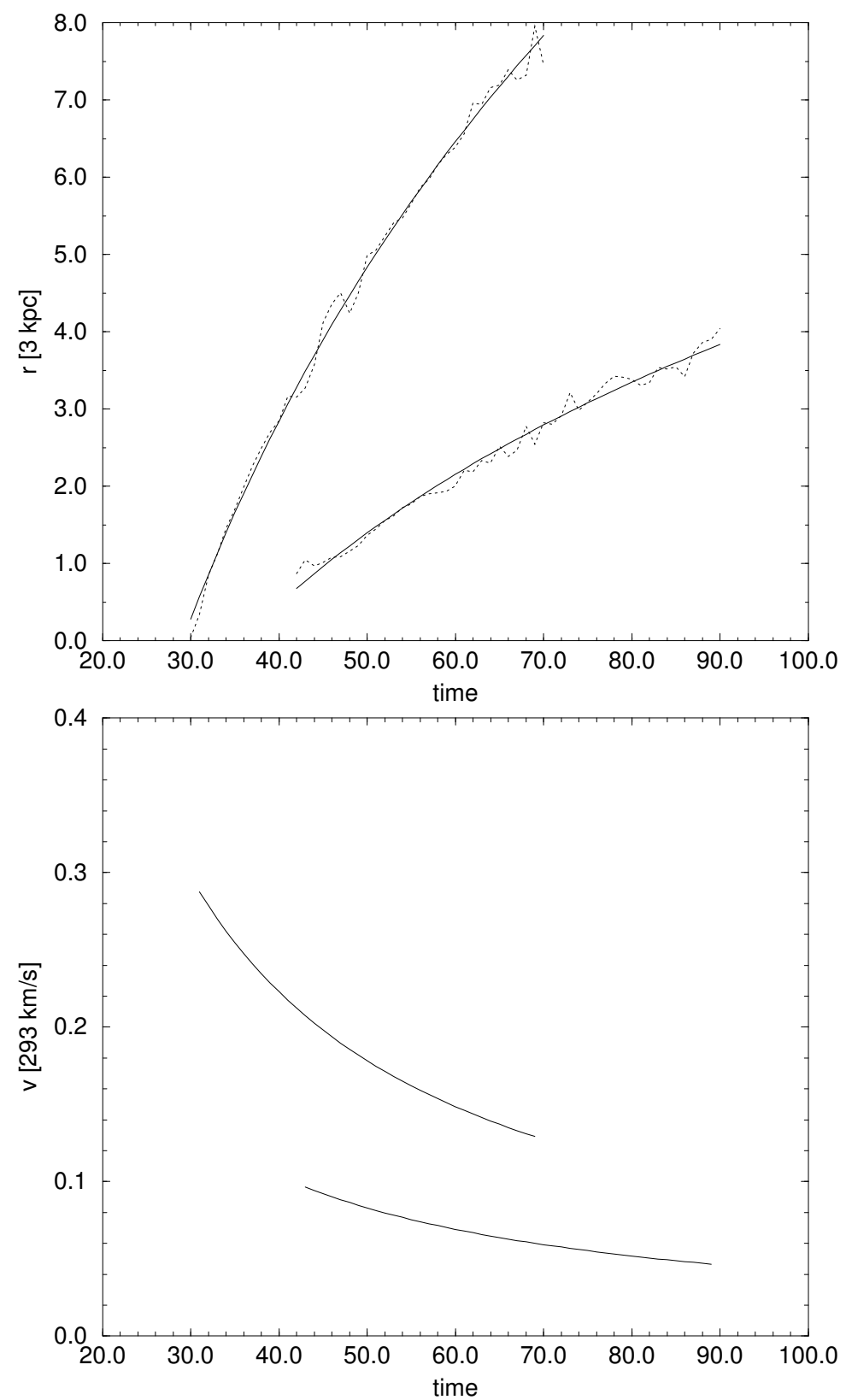

Figure 5.14: Properties of the gaseous rings in model $C 2$. The upper panel shows the radius of the gaseous rings as a function of time. The 'raw' data are given by dotted lines and the fitted function by full lines. The lower panel shows the derivative of the fitted function.

appearance is a projection effect resulting from the different vertical position of the outer, still unperturbed, gas disc and the expanding ring. As a consequence of the 

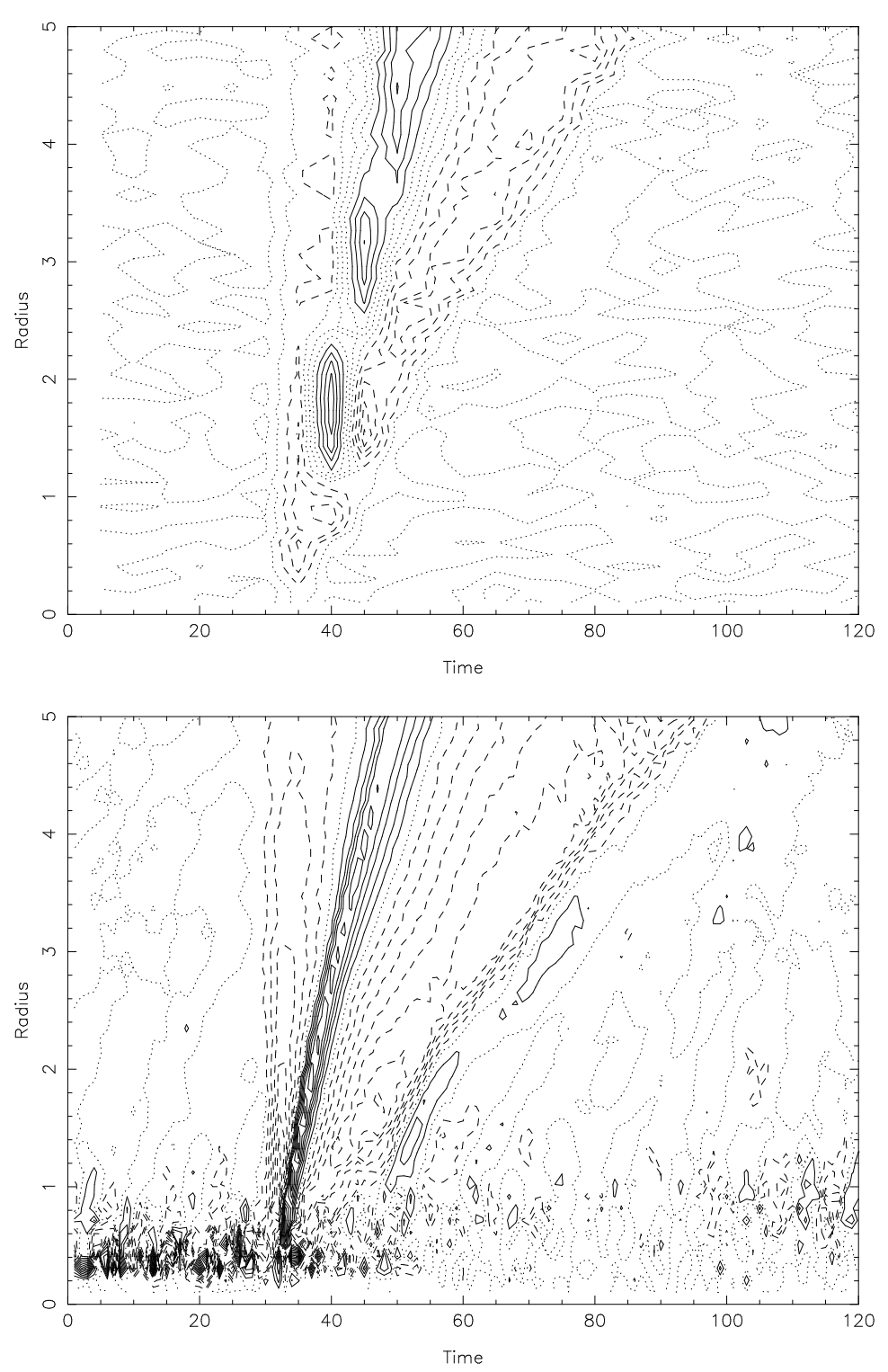

Figure 5.15: Isocontour plots of the radial velocity for the stellar (upper panel) and gaseous (lower panel) disc. Dotted contours correspond to $-10,0$ and $10 \mathrm{~km} \mathrm{~s}^{-1}$, solid line contours to $20,30,40, \ldots \mathrm{km} \mathrm{s}^{-1}$ and dashed ones to $-20,-30,-40, \ldots$ $\mathrm{km} \mathrm{s}^{-1}$.

vertical bending in the disc, induced by the companion prior to impact, the first expanding ring starts forming above the equatorial $z=0$ plane (Fig. 5.7, $t=155$ ). At larger radii, i.e. at later times, the ring crosses the $z=0$ plane of the disc (defined by the centre of mass of the gaseous disc) driven by the passage of the companion. With each passage of a ring through the equatorial disc plane the vertical velocity 
dispersion increases and results in a significant thickening of the disc. The vertical scaleheight of the stellar disc is shown in Fig. 5.5. We find an abrupt increase in $\langle z\rangle$ from a value of roughly $0.6 \mathrm{kpc}$ to $1 \mathrm{kpc}$ at $\Delta t \sim 15$ after the impact.

To analyse the radial and vertical oscillations in the disc in more detail, we trace the orbits of individual particles in $r$ and $z$ as a function of time. With a Fourier transformation we find that the principal frequencies correspond to the radial and vertical epicyclic frequencies in the disc. The increase of kinetic energy in $z$ is therefore responsible for the heating or thickening of the disc after the impact.

\section{Nuclear and circumnuclear disc}

In model C1 (strong bar) the two gas nuclei, which have formed after the interaction in the disrupted bar, merge and form a single nuclear disc. This nuclear disc, however, is less massive and more extended than the one in the isolated model. After the $\mathrm{n}$-disc has formed, it remains connected by two gaseous trailing spiral segments with the second induced ring ( $t=85$ in Fig. 5.6). The gas which is driven towards the centre continuously by way of the spiral shocks accumulates in a circumnuclear disc. Compared to the isolated model, we find about 5 per cent more gas in the cn-disc inside $r=2.0 \mathrm{kpc}$. We also find some two-armed trailing spiral structure of varying strength in the cn-disc. At the end of the run of model $\mathrm{C} 1$, smoothly distributed gas remains in the outer disc, as well as in a dense nuclear disc/core (like in the isolated model) surrounded by a circumnuclear disc with a radius of $r=3 \mathrm{kpc}$ (Fig. 5.4). This disc is orientated almost perpendicular to the nuclear disc and is connected by two trailing spiral arms with an outer distorted ring which has formed at a radius of $r \approx 6 \mathrm{kpc}$, close to the UHR of the former bar (see Fig. 5.8).

In model $\mathrm{C} 2$ the gaseous nuclear disc has already formed before the impact. With the companion passing through the disc, the inner disc region is pulled out of the $z=0$ disc plane towards the direction of the companion. The gaseous nuclear disc reaches a maximum vertical distance relative to the (first) expanding ring of approximately $\Delta z=1.5 \mathrm{kpc}$ at $\Delta t=8$, or $8 \times 10^{7} \mathrm{yr}$, after the impact. After the collisional rings have dissolved and the n-disc has recentred vertically, more gas is driven towards the centre by way of the spiral shocks which have newly formed in the surrounding disc. The radius of the nuclear disc is larger than in the isolated model at the same time, but has about the same mass. The inflowing gas accumulates in a circumnuclear disc, which has a mean radius of $r \approx 2.1 \mathrm{kpc}$ at the end of the simulation and is connected by two spiral arms with the outer ring, which has formed close to the UHR. The region between the cn-disc and the ring at the UHR (at roughly $r \approx 6 \mathrm{kpc}$ ) shows a clear deficiency of gas, which also exists in model C1.

After the bar has been destroyed, the gaseous nuclear discs in both models maintain their slightly elongated shape and still rotate with the same pattern speed as the former stellar bar before the impact. In both models we find spiral shocks in the circumnuclear disc (Fig. 5.4), which persist till the end of the simulation. 

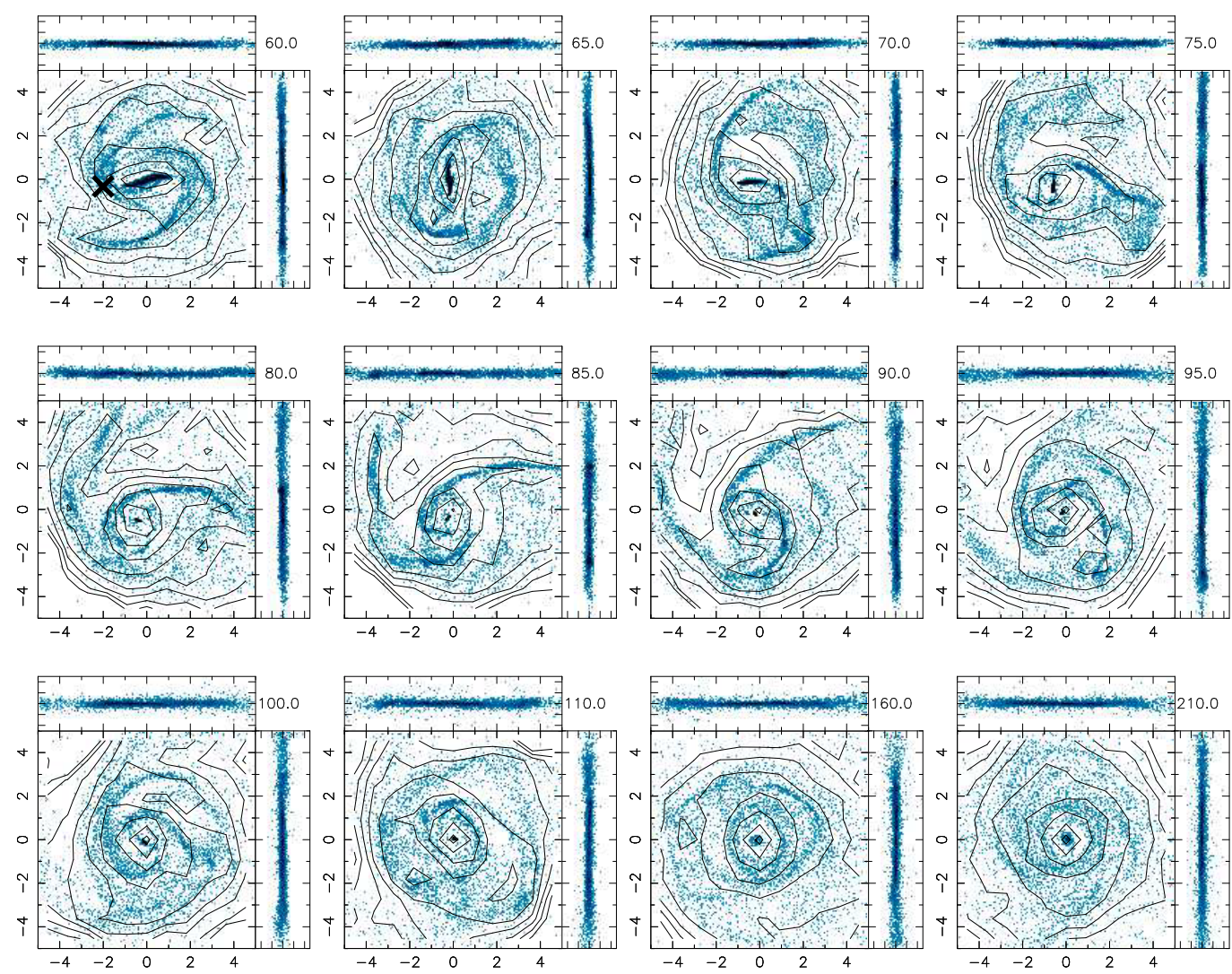

Figure 5.16: Evolution of the stellar and gaseous disc in model $A 1$, where the impact occurs on the bar's major axis at an early time. The layout is as for Fig. 5.2. The origin in the frames is always centred on the centre of mass of the halo component and the impact position of the companion is marked by a cross in the first frame.

\subsubsection{Major axis passages : models $A 1$ and $A 2$}

In these simulations the companion hits the disc on the bar's major axis at a radius of approximately $6.0 \mathrm{kpc}$, i.e. in model A1 close to the end of the strong bar and close to the corotation radius as determined from the isolated model. The morphological evolution of these models is shown in Figs 5.16 and 5.17 for the early (A1) and late (A2) impact, respectively.

When the companion approaches the disc it exerts an extra gravitational force on the disc particles and the bar gets shifted towards the impact position, almost merging with the spiral arm close to the impact position (Fig. 5.16, $t=65$ ). While the central impacts produce a closed expanding ring structure, the off-centred passages produce long spiral arms, which do not form a closed ring (see Fig. 5.16, 

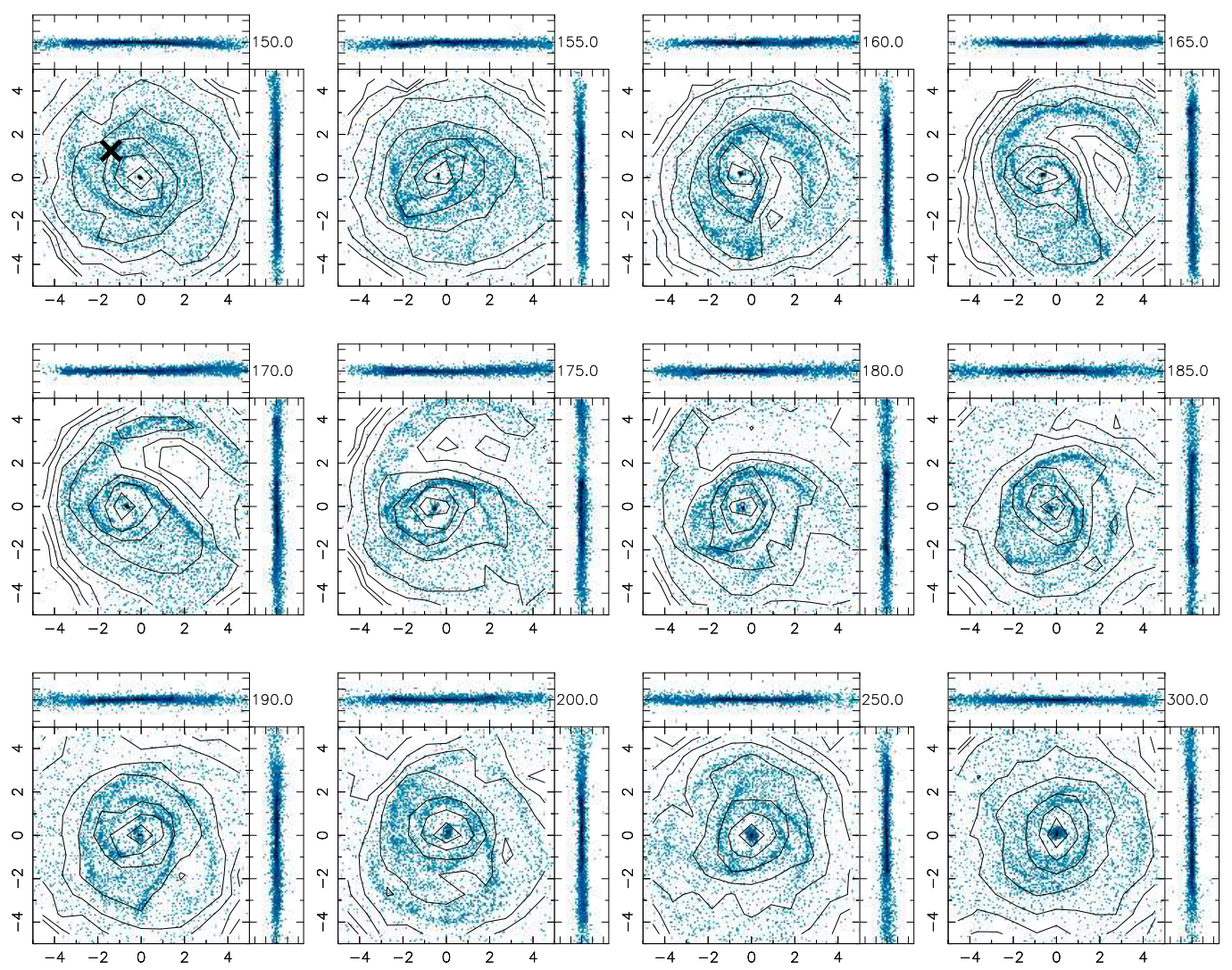

Figure 5.17: Same as Fig. 5.16 but for the late impact model A2.

$t=70$ and Fig. 5.17, $t=165$ ), in good agreement with the results of Toomre (1978) for non-barred galaxies.

\section{Evolution of the bar}

Immediately following the passage of the companion through the disc plane, the stellar bar gets displaced from the centre to a maximum radius of $2.4 \mathrm{kpc}$ in the direction of the impact position in both models A1 (early impact) and A2 (late impact). The off-centring of the bar in these types of interaction has already been described by several authors (see, for example, Gerber \& Lamb1994, 1996; Athanassoula 1996a; APB97). To measure the displacement of the bar we trace its centre of mass iteratively within a cylindrical shell of constant radius and then determine the distance to the centre of mass of the halo, which we can assume to be the dynamical centre of the galaxy. Fig. 5.18 shows the relative distance of the centre of mass of the bar from this centre in the galactic plane as a function of time. In both mod- 


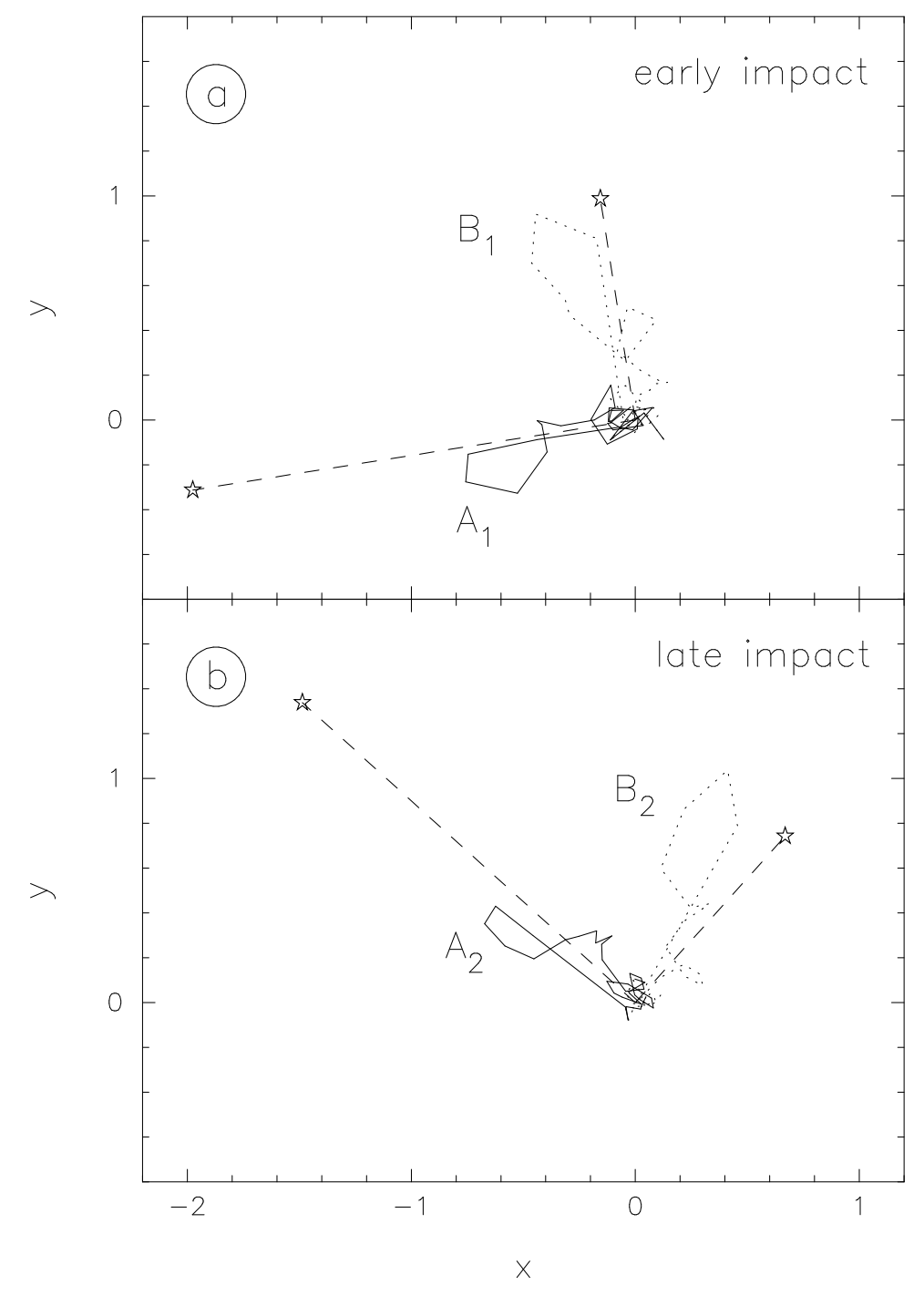

Figure 5.18: Bar displacement. Panels (a) and (b) show the relative distance between the centre of mass of the bar and that of the halo for the early and late impact models, respectively. The stars and dashed lines in each panel mark the impact location in the disc. The models for major and minor axis impacts are shown with full and dotted lines, respectively.

els, the bar, still rotating around its own centre, reaches its maximum distance at $\Delta t=10$ or $0.1 \mathrm{Gyr}$ after the impact and recentres over a period of some two disc rotations, or 0.6 Gyr. The shape of the stellar bar does not change significantly while being off-centred. The bar strength is not affected by the interaction compared to the isolated model (Fig. 5.19), although the bar also weakens owing to gas inflow.

In model A1 the off-centred bar detaches from the ring due to its rotation and 
moves to the centre again. We find that the outer spiral arms in model A2 rotate temporarily with about half of the bar pattern speed for some $0.6 \mathrm{Gyr}$ after the impact. Fig. 5.20 shows the phase angle of the $m=2$ component of the stellar disc mass distribution as a function of radius for different times. The stellar bar detaches from the spiral arms at about $\Delta t=55$ after the impact and reconnects again at $\Delta t=60$. A similar effect has been described by Sellwood \& Sparke (1988; see their fig. 2), who studied the dynamics of an isolated galaxy with different pattern speeds for the bar and the spiral arms. In their models the bar detaches periodically from the spiral arms. In our simulation, however, the bar detaches only once from the spiral arms, because the spiral pattern speed increases again to its initial value of $\Omega=0.3 \tau^{-1}$ after roughly $\Delta t=60$ after the impact. The pattern speed $\Omega_{\mathrm{p}}$ of the bar in model A1 decreases after the interaction for some $0.3 \mathrm{Gyr}$, but reaches its initial value of $0.3 \tau^{-1}$ when the bar has recentred (see Fig. 5.21). The spiral arms in this model rotate with the same pattern speed as the bar and remain connected to it all times.

\section{Nuclear and circumnuclear discs}

In model $A 1$, the gas that was in the shock loci inside the stellar bar before the impact is driven towards the centre of the displaced bar and accumulates in a small elongated nuclear disc, which is aligned with the bar's major axis. At $t=90$ an elongated gaseous ring starts to form with a constant mean radius of approximately $r=4.5 \mathrm{kpc}$, located close to the bar's UHR. The ring and the nuclear disc are connected by spiral arms, which form at about $t=100$. By way of the spiral shocks more gas is driven towards the centre and accumulates in a circumnuclear disc, which is slightly elongated perpendicular to the stellar bar.

The evolution in model A2 is similar. The gaseous nuclear disc is already present before the impact, and, after the stellar bar has recentred, spiral shocks between the nuclear disc and a gaseous ring that has formed close to the UHR drive more gas to the centre. This gas accumulates in a circumnuclear disc. In both models A1 and A2, we find a small gap between the nuclear and circumnuclear disc with a deficiency of gas. The final gas morphology of the central disc region in both models is shown in Fig. 5.4.

\section{Rings and spiral arms}

As in the central impact models, the passage of the companion through the disc produces a radially expanding density wave in both the stars and the gas, originating from the impact position. However, since the impact in model A1 and A2 is offcentred, the symmetry of the ring wave is broken as soon as it encounters the stellar bar and spiral arms in the disc. This is illustrated for model A2 in Figure 5.22. An expanding ring-like structure forms at the impact position, but gets sheared out by the differential rotation in the disc as it expands, forming a pocket structure as 


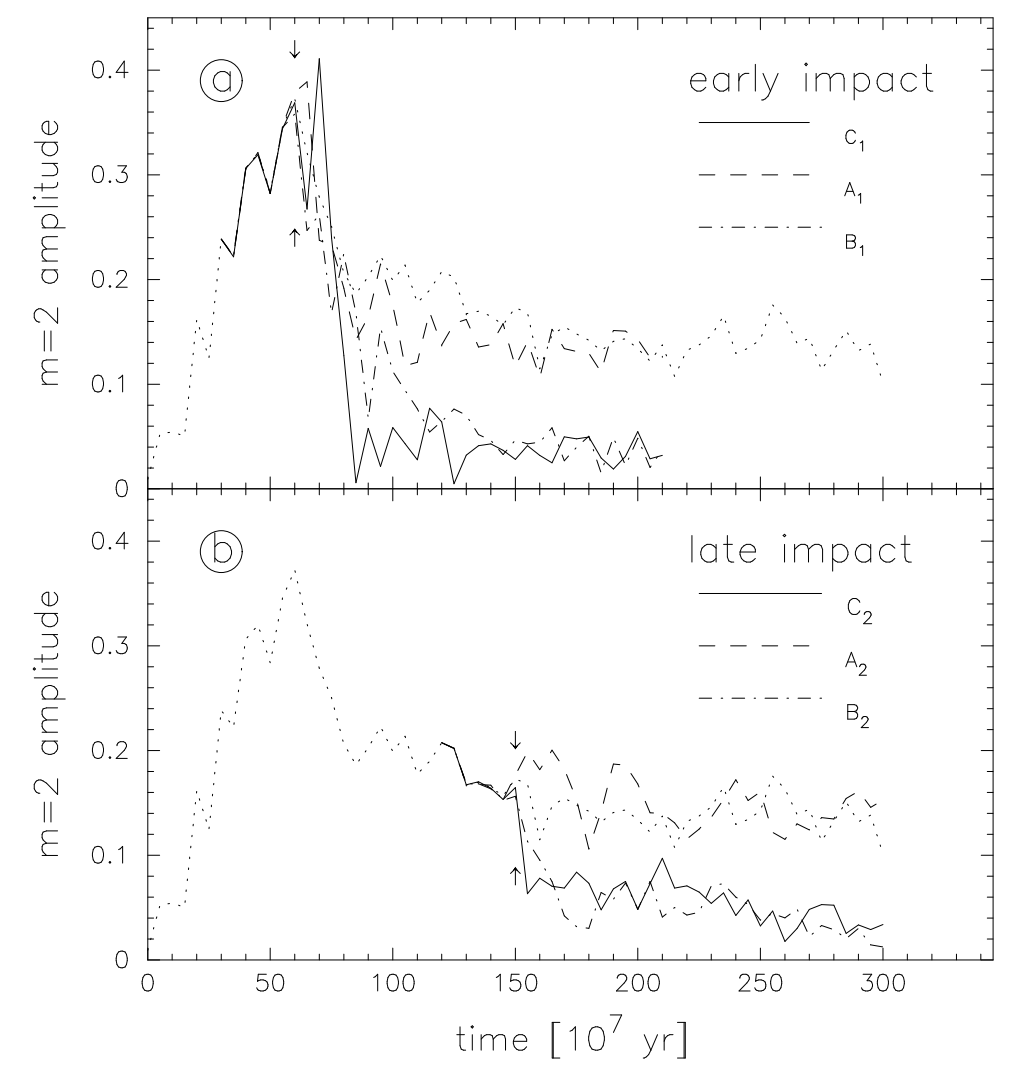

Figure 5.19: Bar strength as a function of time. Panel (a) and (b) show the $m=2$ component for the models with early and late impacts, respectively. The isolated model is shown in both panels (dotted line). The impact time is marked with arrows.

mentioned by Gerber \& Lamb (1994), who studied the caustics produced by an offcentred impact in a non-barred disc model. Because the mass distribution in the disc is more symmetric than in model $A 1$, the features produced are more symmetric. Inside the ring or pocket there is a deficiency of gas. The density wave expands out more freely in the direction of disc rotation. When it encounters the spiral arm, it joins it to form one long tidal arm, which is connected to the end of the off-centred bar (see, for example, Fig. 5.16, $\Delta t \approx 15$ after the impact).

In both models a second expanding density wave starts to form at $\Delta t \approx 20$ after the impact, starting from the impact position in the rotating frame of the disc. When the density wave merges with the spiral arm, a tidal arm is formed which is more linear than the first tidal arm that previously formed on the opposite side of the bar. By this time the first tidal arm in model A1 already extends out to a radius of $r=18 \mathrm{kpc}$, exceeding the initial disc radius. Between the two tidal arms there is a clear deficiency of stars and gas. Both tidal arms wind up and dissolve slowly with most of their material redistributed in the inner $15 \mathrm{kpc}$ again. Some material, 


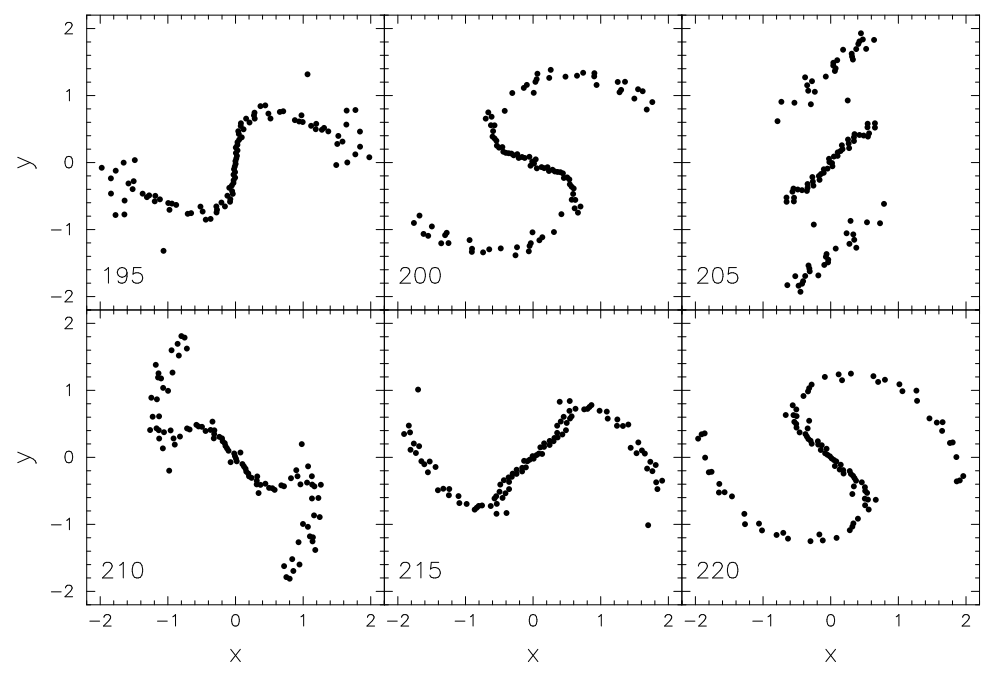

Figure 5.20: Phase angle of the $m=2$ component of the stellar mass distribution as a function of radius for model A2. Note that the outer spiral arms rotate slower than the central stellar bar. At $\Delta t=60(t=210)$ a leading spiral segment is also present, connecting the bar to the outer spirals.

however, is left in the outer disc, giving it an asymmetric shape.

\subsubsection{Minor axis passages : models $B 1$ and $B 2$}

In this section we describe the evolution of the models in which the companion hits the disc in the direction of the bar's minor axis at a distance of approximately $r_{\mathrm{imp}}=3.0 \mathrm{kpc}$, just outside the bar. The impact times for models B1 and B2 are $t_{\mathrm{imp}}=60$ and $t_{\mathrm{imp}}=150$, respectively. Figs 5.23 and 5.24 show the evolution of both the stellar and the gaseous disc.

Before the impact the evolution of the disc is similar to the isolated model I 0 . In contrast to the central impact cases we do not find a vertical bending of the disc prior to impact in either of the two models. After the impact the bar is temporarily off-centred, and the passage of the companion excites radial oscillations in the disc, resulting in the formation of an expanding density wave. Owing to its off-centring the impact produces two long tidal arms rather than a closed ring structure. Finally, the stellar bar gets almost destroyed by the interactions in both models.

\section{Evolution of the bar}

Immediately following the impact, the stellar bar gets displaced from the centre in both models to a maximum distance of approximately $3.0 \mathrm{kpc}$ towards the impact position, i.e. to a distance comparable with that of the impact location from the 


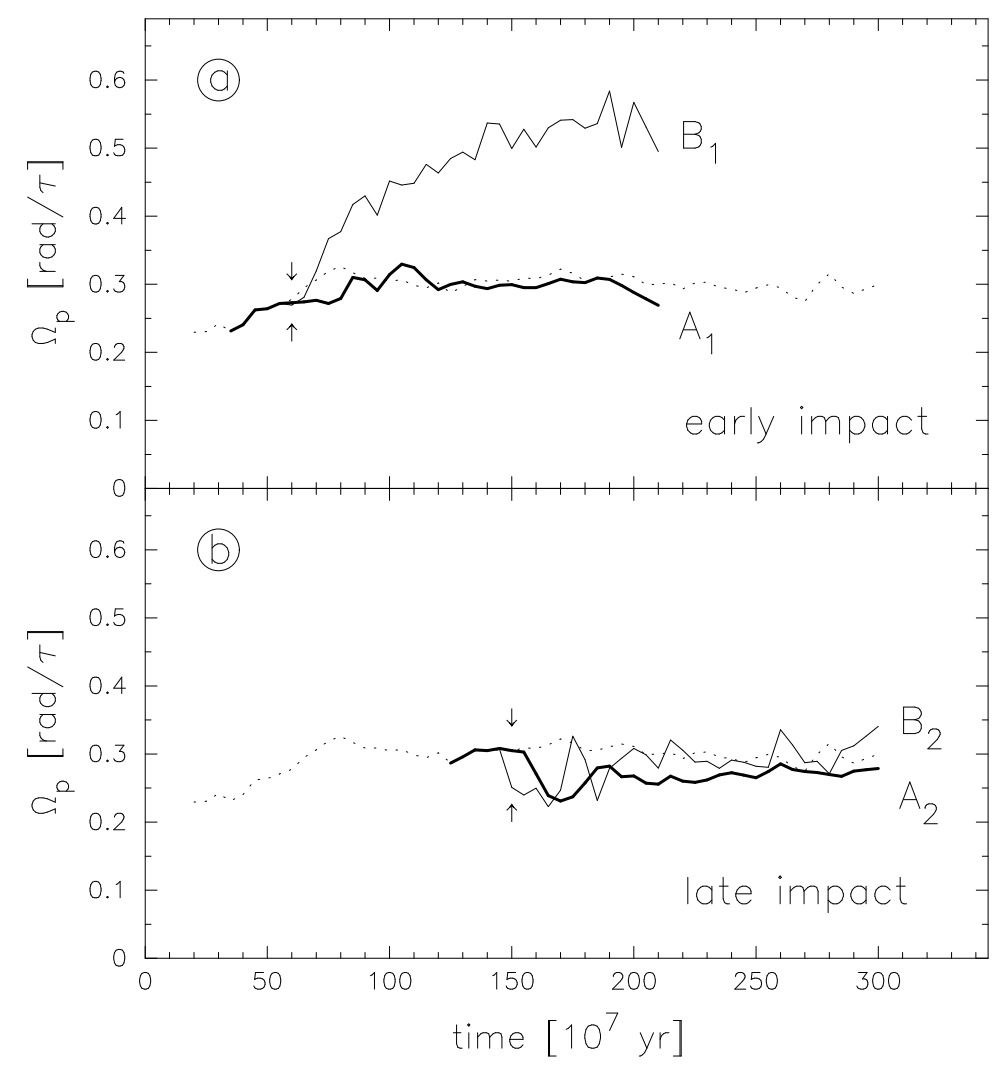

Figure 5.21: Pattern speed $\Omega_{\mathrm{p}}$ as a function of time. Panel (a) shows model A1 (thick line) and B1 (thin line) with the early impact at $t=60$. The dotted line shows the evolution for the isolated model I0. The same for panel (b) for models A2 (thick line) and B2 (thin line) with impact at time $t=150$. The impact times are marked by arrows in both panels.

centre of the target disc (Fig. 5.18). The off-centred bar still rotates around its own centre, while moving radially in the disc. The bar reaches its maximum distance from the dynamical centre at a time of $\Delta t=10$ after the impact of the perturber, which is the same time-scale as in the major axis impact models. The offset of the bar lasts for a period of roughly $\Delta t=0.6 \mathrm{Gyr}$, or about two disc rotations, in both models, as for models $\mathrm{A} 1$ and $\mathrm{A} 2$.

We measure the strength and pattern speed of the bar with the method described in Sec. 5.3.1. Care was taken that no power from spiral features was included in the computation by checking that the phase angle is constant with radius. As the bar strength weakened, the error in the pattern speed measurement increased, but was always below about 5 per cent. While the pattern speed in model B2 remains constant after the impact, we find an increase of $\Omega_{\mathrm{p}}$ in the early-impact model B1 from $0.3 \tau^{-1}$ to a rate of $0.5 \tau^{-1}$ (see Fig. 5.21), presumably owing to the torque 

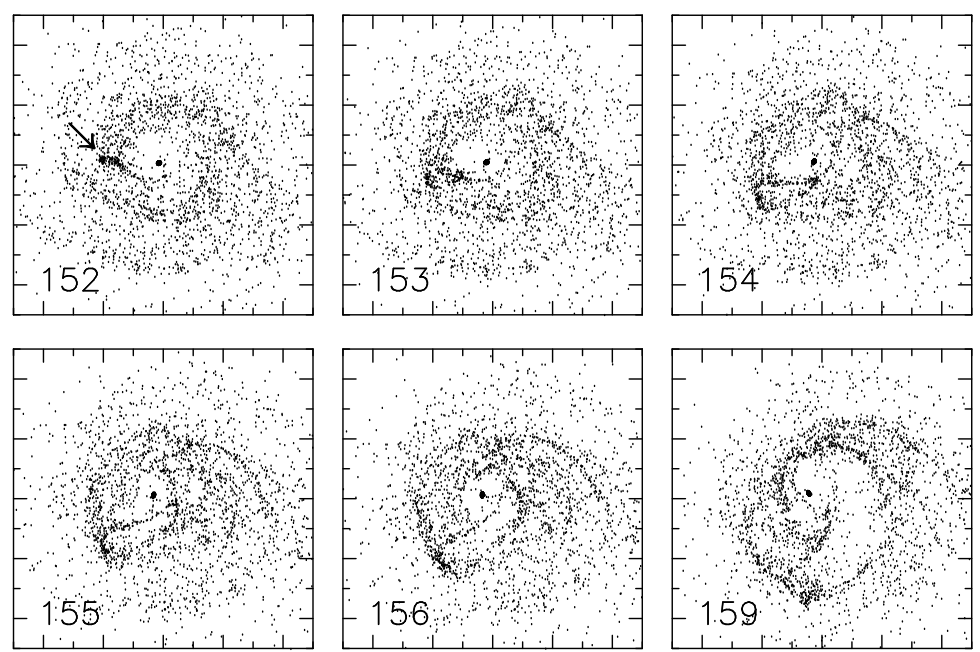

Figure 5.22: Evolution of the gaseous disc in model $A 2$ after the impact. We show the face-on gas particle distribution. The time in model units is given in the lower left-hand corner in each frame. The size of each box corresponds to $30 \mathrm{kpc}$. The position of the forming density wave is marked in the first frame by an arrow.

by the companion on the strong bar. Since the rotation curve of the disc does not change significantly after the impact, the change of $\Omega_{\mathrm{p}}$ is accompanied by a change of the positions of the resonances in the disc. In fact this change can be observed in the resonance gas ring located close to the bar's UHR. While $\Omega_{\mathrm{p}}$ increases, the UHR moves inward and the ring shrinks from a radius of approximately $r=3 \mathrm{kpc}$ to $2.4 \mathrm{kpc}$, compatible with the change of $\Omega_{\mathrm{p}}$.

By the end of the simulation, the stellar bar is almost destroyed by the interactions in both models. However, the dissolution time-scale for the early-impact case B1 is longer than in the corresponding model with central impact (Fig.5.19). There is an indication that this might also be true, but to a lesser degree, for the lateimpact case B2. The vertical scaleheight of the disc, however, does not increase considerably after the interaction (see Fig. 5.5).

\section{Nuclear and circumnuclear disc}

In model B1, where the bar is strong at impact time, the leading gaseous shocks remain inside the off-centred stellar bar. As the bar is recentring, the gas flows toward the centre of the bar. Following the bar recentring this gas forms a dense nuclear disc which is aligned with the bar's major axis. Over time the nuclear disc looses its elongated shape and becomes more circular, while the inflow rate inside a central region of $450 \mathrm{pc}$ remains constant at about $4 \mathrm{M}_{\odot} \mathrm{yr}^{-1}$. The total amount of gas driven towards the centre, which is limited by the amount of gas within the 

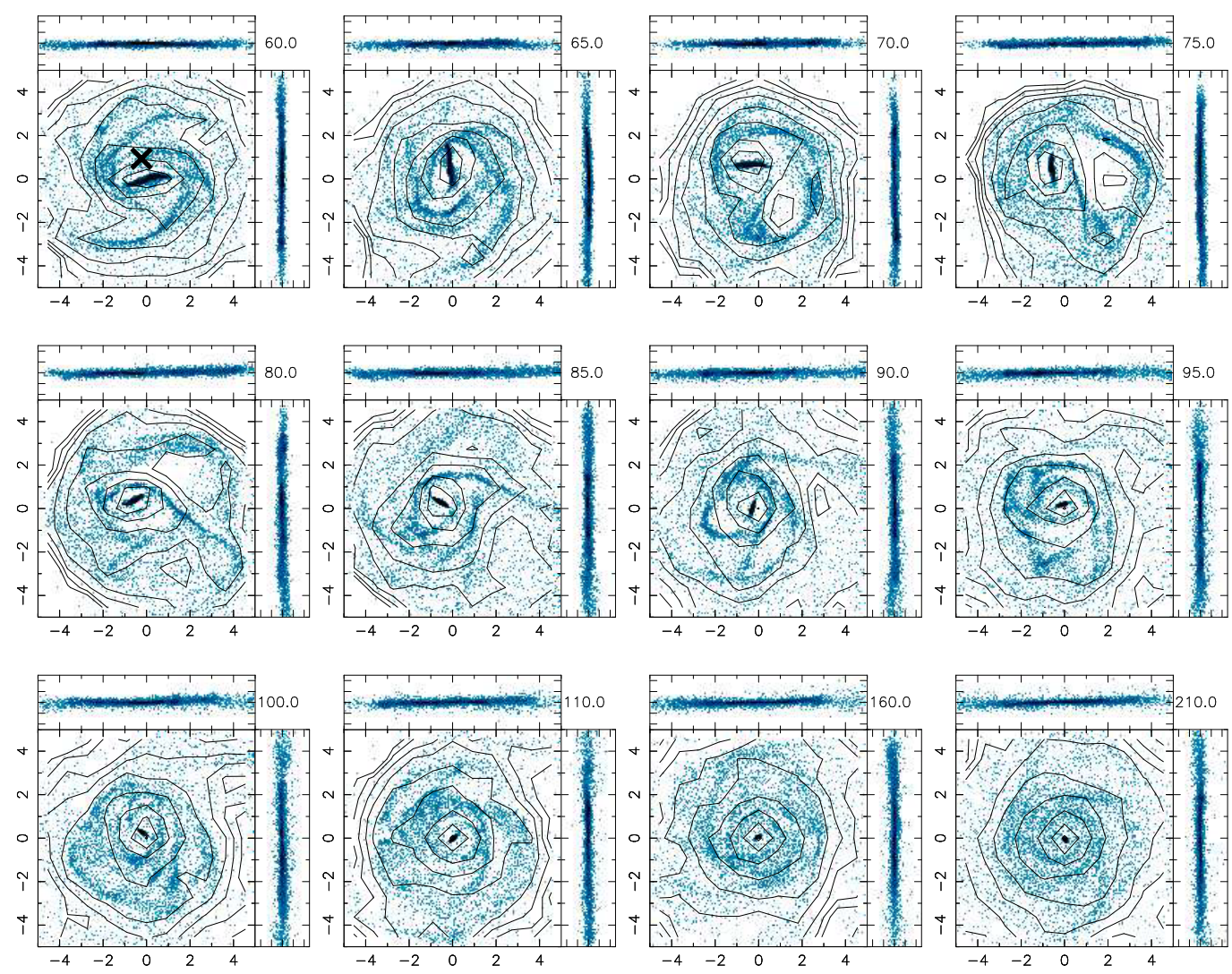

Figure 5.23: Evolution of the stellar and gaseous disc in model $B 1$, where the impact occurs on the bar minor axis at an early time. The layout is as in Fig. 5.16.

bar region before the impact, is the same as in the isolated model. In this model, however, no circumnuclear gaseous disc forms (see Fig. 5.4).

The nuclear disc in model B2 has already formed before the impact and remains in the centre of the temporarily off-centred bar. Some of the inflowing gas accumulates in a large diffuse circumnuclear disc, almost extending out to the gas ring at $3 \mathrm{kpc}$, which has formed following the impact.

\section{Rings and spiral arms}

The passage of the companion through the disc produces an expanding density wave, which becomes visible first in the gas. The general evolution of this ringwave is similar to the one in the major axis impact models. As can be seen in Figs 5.23 and 5.24 the density wave expands more freely in the direction of disc rotation and maintains longer its circular shape on that side ( $\Delta t=5$ after the impact) 

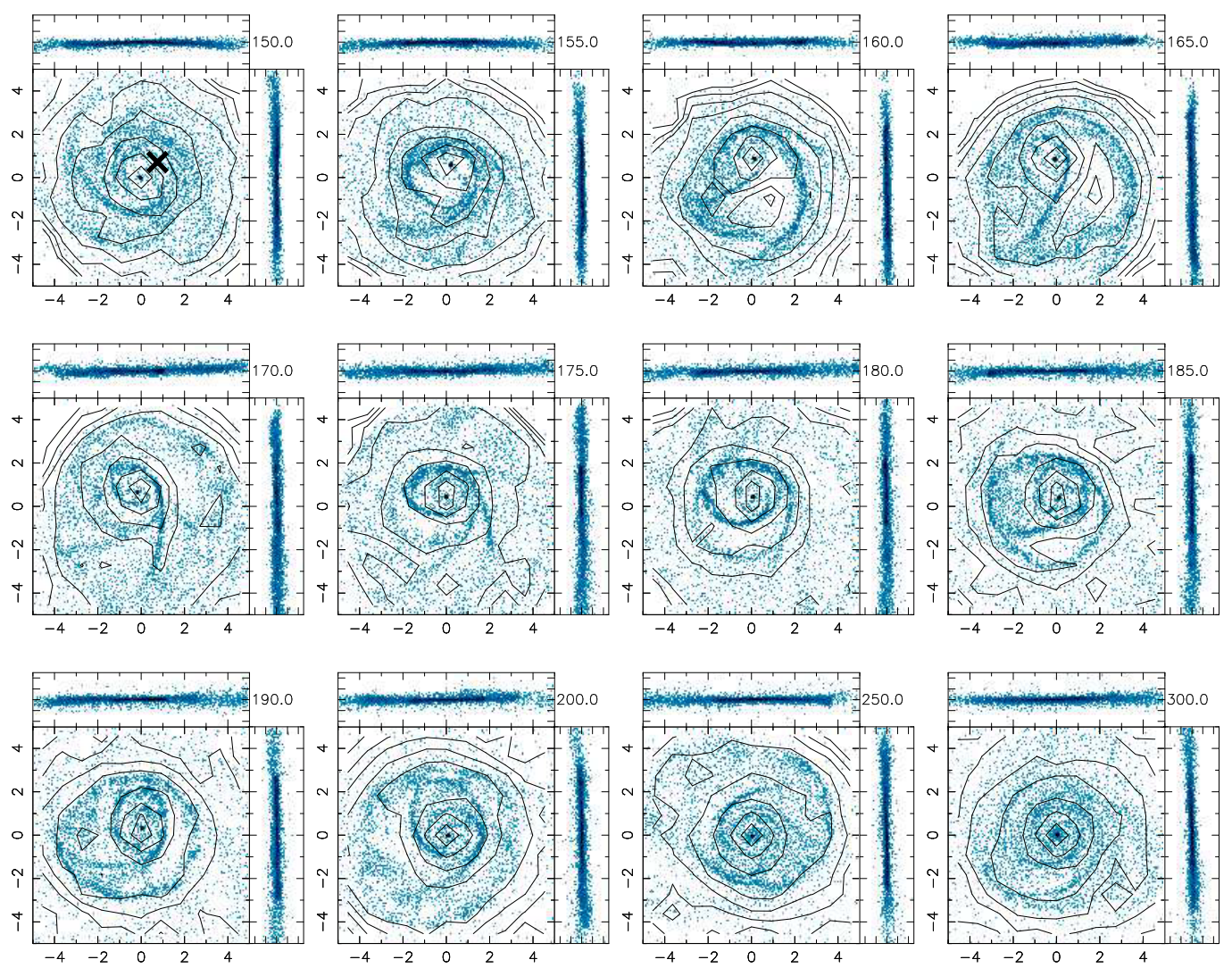

Figure 5.24: Same as Fig. 5.23 but for the late-impact model B2.

before merging with the spiral arm in the outer disc. As soon as the wave encounters the spiral arm, the ring feature opens up and forms a long tidal arm, extending out to a maximum radius of approximately $r=18 \mathrm{kpc}$. Then the tidal spiral arm dissolves slowly and most of its material is redistributed in the initial disc region. The other part of the ring remains close to the off-centred bar and is connected to it by a straight spoke $(\Delta t=10)$. Between the bar and the spoke, which is visible in both the stars and the gas, there is a clear deficiency of gas.

In both models a second expanding density wave ring becomes visible $\Delta t=20$ after the impact, when the bar has almost recentred. The second ring in model B1 merges with a spiral arm segment, which has formed after the impact (Fig. 5.23). In B2 the second ring is more symmetric than the first one and encloses the weak bar completely. However, the ring is not centred with respect to the bar. In fact the bar remains connected to the ring for a few dynamical times (Fig.5.24). Similar to the previous simulations, additional ring-like structures or segments form in the gas at later times, merging with the spiral arms in the disc. These features are not 
supported by a stellar counterpart and are washed out relatively fast.

\subsection{Discussion}

\subsubsection{Bar amplitude}

The evolution of the bar strength for the different models is shown in Fig. 5.19 (a) and (b). In the isolated model the stellar bar forms within a few disc rotations and reaches its maximum strength at $t=60$. As a result of the gas inflow the amplitude decreases rapidly at first, but then settles down to a quasi-stationary state, in which its strength decreases linearly on a time-scale which is significantly longer than the dynamical one, as described in Sec. 5.3.1.

The passage of the companion through the disc has several effects on the morphological evolution of the bar, such as a temporary off-centring and a weakening or destruction of the bar. The strength of these effects depends strongly on the impact location relative to the bar. With the central passage the bar is destroyed very shortly after the impact of the companion ( $\Delta t=10$, or 0.5 disc rotations). For the early impact, the bar gets torn into two fragments that move outward with the expanding ring and later merge again, while for the late impact, no bar is left after the first stellar ring becomes visible. In both models the destruction of the bar is accompanied by a considerable vertical thickening of the disc. Impacts on the bar's major axis (models of type A) show a similar evolution of bar strength as in the isolated model, although the bar is temporarily off-centred after the impact for some 0.6 Gyr. Comparison with the isolated model shows that the bar strength in these models is more significantly affected by the gas inflow than by the interaction itself. When the companion is close to the $z=0$ disc plane, the tidal force it exerts on the bar is directed almost parallel to the bar major axis and thus does not disrupt the bar structure much. In the models with the minor axis passage (models B1 and B2) the bar is nearly destroyed, but its amplitude decreases more slowly $(\Delta t=50)$ than in the corresponding central passage, an effect which is particularly evident in model B1. The decay of the bar amplitude in both models can be described as quasi-exponential. The dissolution of the bar is not accompanied by a vertical thickening of the disc in these models. For the minor axis impacts it seems to be possible to destroy the stellar bar, while keeping the disc, in contrast to the pure $N$-body simulations where it is found that an interaction sufficiently strong to destroy the bar also destroys the disc (Athanassoula 1996b), i.e. the ratio between the vertical and the radial scaleheights increased very significantly. This could be due to the fact that the decrease in the bar amplitude in the purely stellar cases can be due only to the impact, while in simulations including a gaseous component it is due to the cummulative effects of the impact and the gaseous central mass concentration.

Central impacts and passages through the bar's minor axis seem to cause more damage to the bar than the major axis impacts. The disc thickens more if it is 
hit at the centre than at the periphery, so the disc of models of type $\mathrm{C}$ thickens considerably, while models of type A and B do not show a significant difference in this regard. Between early- and late-impact models with same impact positions, we find differences in the bar's evolution shortly after the impact because of the different initial state of the bar at impact time, but the long-term evolution of the bar in both types of models is similar. We conclude that the fate of the bar is more sensitive to the impact location of the companion relative to the bar than to the evolutionary phase of the bar during impact. In general, the impact of a companion tends to accelerate the evolution from a strongly-barred spiral to a weakly- or nonbarred galaxy, a trend also found in the isolated model, but on longer time-scales.

\subsubsection{Bar pattern speed}

The evolution of the pattern speed in the isolated model is mainly influenced by two processes. Angular momentum is transferred to the outer disc and halo resulting in a decrease of the pattern speed (see, for example, Tremaine \& Weinberg 1984; Weinberg 1985; Little \& Carlberg 1991; Hernquist \& Weinberg 1992; Athanassoula 1996b, 2002; Debattista \& Sellwood 1998, 2000), while mass accretion into the nuclear region steepens the rotation curve at the centre and leads to an increase of $\Omega_{\mathrm{p}}$ (see, for example, Friedli \& Benz 1993; Heller \& Shlosman 1994; Berentzen et al. 1998). In our isolated model we find that during the early phases of the bar evolution, i.e. during its growth and dissolution before it has reached quasi-stability, the pattern speed increases linearly from $\Omega_{\mathrm{p}}=0.22$ at $t=30$ to $\Omega_{\mathrm{p}}=0.30$ at $t=80$, and thereafter stays constant to the end of the run. This behavior is in agreement with other numerical simulations which have included a gas component (e.g., Friedli \& Benz 1993; Heller \& Shlosman 1994; Berentzen et al. 1998), showing either constant or slightly increasing pattern speeds.

Owing to the interaction, for models with impacts on either the major or the minor axis of the bar we find some variations in the pattern speed (see Fig. 5.21). In the late-impact models, shortly after the impact, the pattern speed decreases temporarily by about 30 per cent, but when the bar recentres again $\Omega_{\mathrm{p}}$ increases back to its original value, though with oscillations that are damped over time. One of our models, however, shows an evolution in pattern speed that is different from that of our other vertical-impact models. In model B1, where the companion hits the disc on the minor axis of the strong bar, we find an increase of $\Omega_{\mathrm{p}}$ from $0.3 \tau^{-1}$ to $0.5 \tau^{-1}$. The bar maintains this high rotation after recentring and so has gained angular momentum. This effect is probably due to torques exerted by the perturber galaxy on the bar. For the central passage models $\mathrm{C} 1$ and $\mathrm{C} 2$ the bar seems to be slowed down by the interaction, although the $m=2$ amplitude of the bar is only marginal and a pattern speed is difficult to measure with confidence. The collisionless models studied by Athanassoula (1996a) and APB97 showed a decrease of the bar pattern speed in those models where an abrupt change of $\Omega_{\mathrm{p}}$ occurred after the impact. Sundin et al. (1993) found that depending on the mass of the perturber, 
both an increase or a decrease of $\Omega_{\mathrm{p}}$ was possible. In their models, however, the trajectory of the perturbing galaxy lies in the equatorial plane of the disc.

\subsubsection{Off-centred bars}

Another important feature produced by the interaction is the displacement of the bar from the centre of the galaxy. Asymmetries of this type are frequently found in disc galaxies, particularly in late-type galaxies (see, for example, de Vaucouleurs \& Freeman 1972; Odewahn 1996). While this kind of asymmetry could be produced by strong $m=1$ modes in the disc, an off-centred impact of a companion galaxy should generally give rise to more significant shifts, depending on the impact parameters. In all our models with off-set passages we notice a strong displacement of the bar structure for approximately $0.6 \mathrm{Gyr}$, independent of the evolutionary phase of the bar at impact time. The maximum displacement of the bar centre, which is in our models roughly $\Delta R=3 \mathrm{kpc}$, depends only on the impact position or distance of the companion from the bar centre, but not on the strength of the bar at impact time. The size of the displacement in our models is in good agreement with the relative displacement parameter found by Feitzinger (1980) for Magellanic type galaxies. The off-centring of the bar is also accompanied by asymmetries in the spiral structure. The morphology of the discs with off-centred bars in our simulations is dominated by one long spiral arm, which formed after the impact by an expanding density wave, in agreement with the results of Athanassoula (1996b) and with the morphology of several asymmetric barred galaxies, such as NGC 4027. The induced asymmetries are visible in the disc, for about $0.6 \mathrm{Gyr}$ after the impact.

\subsubsection{Nuclear and circumnuclear disc}

The presence of the bar results in a considerable change in the mass distribution of the gas. Owing to the bar torques, gas is driven towards the centre of the galaxy and accumulates in nuclear and circumnuclear discs. This type of discs forms in both the isolated and the interacting models, although its shape and size varies depending on the interaction parameters (Fig. 5.4). The circumnuclear disc is always elongated perpendicular to the major axis of the bar, indicating that the gas in it populates the $x_{2}$ orbits and is therefore trapped between the inner and the outer ILR, which have been confirmed to be present by the surfaces of section. The gas in the nuclear disc represents some 18 per cent of the total dynamical mass inside the central region, i.e. within a radius of $1 \mathrm{kpc}$. This is about the same fraction as found in the isolated barred galaxy model by Berentzen et al. (1998).

The cn-discs in the interacting models with central impact are much larger than in the isolated model (up to a factor of three in radius). Since the bar is destroyed by the interaction almost immediately in these models, no torque acts on the gas to drive it closer to the centre. In the major axis impacts, the bar keeps roughly the same strength as in the isolated model, therefore we do not find a significant 
difference in the morphology of the central region. Very little additional gas is driven towards the centre by the impact itself.

\subsubsection{Rings and spokes}

In our central impact models $\mathrm{C} 1$ and $\mathrm{C} 2$, following the passage of the companion, we find expanding ring structures in the disc. The process of ring formation by interactions is described for non-barred galaxies in detail by Lynds \& Toomre (1976). These authors show that the expanding stellar ring is a density wave feature travelling through the disc and is due to radial oscillations of the disc particles, as later confirmed by simulations (see, for example, APB97). We find such radial oscillations for both the stellar and the gas particles, of which the latter contribute longer to the rings. The gaseous ring always remains within the expanding stellar ring, having roughly the same radius but considerably smaller width. The gaseous rings fragment on the inner side owing to the self-gravity of the gas and its dissipative nature, while gas piles up in the ring during its expansion, because the gaseous orbits cannot cross. In contrast, the stellar ring becomes weaker and broader while expanding, but the gas ring can be identified for a longer time.

The expansion velocity of the rings decreases with radius as predicted by the impulse approximation and as found in $N$-body simulations (APB97). The expansion velocity of the first ring drops gradually from $88 \mathrm{~km} \mathrm{~s}^{-1}\left(7.5 v_{\mathrm{s}}\right)$ to $35 \mathrm{~km} \mathrm{~s}^{-1}$ ( $3 v_{\mathrm{s}}$, where $v_{\mathrm{s}}$ is the sound speed in the gas). With the passage of the ring the local velocity dispersion in the disc increases. The second ring in our models starts off more slowly than the first, with an expansion velocity which decreases from 29 $\mathrm{km} \mathrm{s}^{-1}\left(2.5 v_{\mathrm{s}}\right)$ to $11.72 \mathrm{~km} \mathrm{~s}^{-1}\left(1 v_{\mathrm{s}}\right)$. Note that there is a transition in the gas from a supersonic density wave supported by the stellar rings to an acoustic wave.

A well-known example of a collisionally-induced ring galaxy is the Cartwheel galaxy (A0035-324). Density enhancements like the rings are accompanied by the formation of bright young blue stars and $\mathrm{H}$ II regions, while the induced stellar ring represents mainly the underlying disc population. The star-formation rate found in the outer ring of the Cartwheel galaxy is about $6.7 \times 10^{7} \mathrm{M}_{\odot} \mathrm{Myr}^{-1}$ (Higdon 1995). After the passage of such star-forming rings, colour gradients are expected in the disc and have been observed in several ring galaxies (Korchagin et al. 1999). In particular, such radial age gradients have been found in the optical and the NIR in the Cartwheel galaxy (Marcum, Appleton \& Hidgon 1992). In numerical simulations that have included star formation (Mihos \& Hernquist 1994), however, such gradients remained for only a short period of time before the nuclear starburst dominated the rings. In our model $\mathrm{C} 2$ we find that the second gas ring, which forms after the impact, consists mainly of particles that had already contributed to the first one. Thus, if we assume that some fraction of this gas is used up by star formation, we might expect the inner ring to contain considerably less gas than the outer ones, as confirmed by the observations of Cartwheel. 


\subsubsection{Role of the halo}

To study the role of the halo response in our simulations, we rerun a simulation of model C2 but with a fixed (frozen) halo, i.e. we represented the halo by an external force, unchanged through the simulation. We find that the morphological evolution of the disc in face-on projection is very similar to the case with a live halo, e.g. the number of rings formed after the impact, their shape and their expansion velocity are qualitatively reproduced. The main difference between the two models is found in the vertical structure of the disc following the impact. In the edge-on projection we find that in the frozen halo model both the gaseous and the stellar disc become vertically thicker after the impact and settle down in a more irregular shape, i.e. not disc-like, in contrast to the live-halo model. In the latter we find that the halo gets centrally less concentrated and dynamically hotter after the impact. We therefore conclude that the live halo stabilizes the disc by absorbing energy from the impact.

\subsection{Summary}

We have performed fully self-consistent $N$-body/SPH simulations to study the interaction between an initially barred galaxy and a less massive spherical companion. The companion passes almost vertically through the disc of the host galaxy and leaves the host system following the impact. The mass of the companion has been chosen so as to be a significant perturbation to the disc. Two different impact times have been chosen, representing different characteristic phases of the isolated bar's evolution. In the first set of simulations the impact is at an early phase of evolution when the bar is strongest, while in the second set an advanced phase has been chosen, i.e. when the bar is weak and has settled down in a quasi-stable state. Beside the impact time, we also varied the position of the impact with respect to the bar in the disc. In the simplest case the companion hits the bar at its centre, while in other cases it hits the disc outside the bar at its major or minor axis. Interactions at times before a stellar bar has formed or during its non-linear growth phase have not been performed here.

The interactions produce characteristic features that remain in the disc for only a few dynamical times. In general the gaseous features tend to persist longer than their stellar counterparts, though after about $1 \mathrm{Gyr}$ both of them are dissolved. The most prominent features produced by the interaction are the collisionally induced rings, which are well known from non-barred galaxies. The central impacts produce expanding stellar and gaseous rings whose symmetry is disturbed in the case of the strong-bar model. The stellar ring is a density wave produced by forced radial oscillations of the stellar orbits. The basic properties of the stellar rings, such as density or expansion velocity, are well described, at least qualitatively, by the impulse approximation. The gaseous rings also show the characteristics of a density wave, which is supported by the potential of the stellar ring. We find that more 
than two rings can be formed in the gaseous disc by the impact, some of them not supported by stellar density enhancements. These rings are less pronounced than the first two rings and dissolve much faster. In contrast to central impacts, the offcentred passages produce outwardly expanding ring-like density waves - not in the form of closed rings, but as long tidal spiral arms whose extent exceeds the initial disc cut-off radius. These tidal arms are present for some few dynamical times in our simulations, before they dissolve and the material in the arms is redistributed in the disc.

In the simulations with off-centred impacts, the stellar bar gets temporarily displaced from the centre of the galaxy for some $0.6 \mathrm{Gyr}$ before it recentres again. The gas inside the bar is driven towards the centre, forming a nuclear disc whose mass is determined by the amount of gas located in the bar region. Almost no additional gas is driven towards the centre of the galaxy owing to the interaction, compared with the isolated model. In most of our simulations, the stellar bar is destroyed after the impact of the companion, leaving behind a dense stellar core at the centre of the galaxy. In the central impact models the destruction of the bar is accompanied with a considerable thickening of the disc. In models with minor axis impacts, however, it seems to be possible to destroy the bar while keeping the disc. We also find cases (i.e., the major axis impact) in which the bar survives the interaction, resembling the weak bar of the isolated model. We argue that the interaction with a companion can drive the transition from a strongly-barred galaxy to a weakly-barred galaxy on shorter time-scales than those found in isolated models. The impact time, or the dynamical phase of the disc, does not play a significant role in determining the final morphology of the disc. The dense nuclear disc and the surrounding gap in the gas distribution are the only morphological imprints that survive the interaction, even after the bar has dissolved. Such features in a non-barred galaxy may indicate the former existence of a bar. Apart from that, looking at the final face-on morphology of the gaseous disc - in which the differences are more clear than in the stellar - the interacting and non-interacting models can hardly be separated. This might be possible from models including star formation, e.g. from the resulting colour/metallicity gradients.

\subsection{Acknowledgments}

We would like to thank Albert Bosma for interesting discussions, J.C. Lambert and C. Theis for their computer assistance and an anonymous referee for comments which helped to improve the presentation. IB acknowledges DFG grant Fr 325/481, /48-2 which supported this work and the GRAPE facilities at the Sternwarte Göttingen. $\mathrm{CH}$ acknowledges support from DFG grant Fr 325/39-1, /39-2. EA would like to thank the region PACA, the IGRAP, the INSU/CNRS and the University of Aix-Marseille I for funds to develop the GRAPE facilities used for part of the calculations in this paper. 


\subsection{Bibliography}

Athanassoula E., 1992a, MNRAS, 259, 328

Athanassoula E., 1992b, MNRAS, 259, 345

Athanassoula E., 1996a, in Buta R., Crocker D.A., Elmegreen B.G., eds, ASP Conf.

Ser. Vol. 91, Barred Galaxies. Astron. Soc. Pac., San Francisco, p. 309

Athanassoula E., 1996b, in Sandquist Aa., Lindblad P.O., eds, Lecture Notes in

Physics Vol. 474, Barred Galaxies and Circumnuclear Activity. Springer Verlag,

p. 59

Athanassoula E., 1999, in Sellwood J.A., Goodman J., eds, ASP Conf. Ser. Vol.

160, Astrophysical Discs. Astron. Soc. Pac., San Francisco, p. 351

Athanassoula E., 2002, ApJL, 569, 83

Athanassoula E., Bienayme O., Martinet L., Pfenniger D., 1983, A\&A, 127, 349

Athanassoula E., Puerari I., Bosma A., 1997, MNRAS, 286, 284 (APB97)

Barnes J.E., Hernquist L., 1992, ARA\&A, 30, 705

Barnes J.E., Hernquist L., 1996, ApJ, 471, 115

Berentzen I., Heller C.H., Shlosman I., Fricke K.J., 1998, MNRAS 300, 49

Binney J., Tremaine S., 1987, Galactic Dynamics. Princeton Univ. Press

Contopoulos G., Mertzanides C., 1977, A\&A, 61, 477

Contopoulos G., Papayannopoulos, 1980, A\&A, 92, 33

Debattista V.P., Sellwood J.A., 1998, ApJ, 493, 5

Debattista V.P., Sellwood J.A., 2000, ApJ, 543, 70

de Vaucouleurs G., Freeman K.C., 1972, Vistas in Astronomy, 14, 163

Eskridge P.B. et al., 2000, AJ, 119, 536

Feitzinger J.V., 1980, Space Sci. Review, 27, 35

Friedli D., Benz W., 1993, A\&A, 268, 65

Gerber R.A., Lamb S.A., 1994, ApJ ,431, 604

Gerber R.A., Lamb S.A., 1996, MNRAS, 278, 345

Gerin M., Combes F., Athanassoula E., 1990, A\&A, 230, 37

Heller C.H., 1991, PhD thesis, Yale Univ.

Heller C.H., Shlosman, I., 1994, ApJ, 424, 84

Hernquist L., Weinberg M.D., 1992, ApJ, 400, 80

Hernquist L., Weil M.L., 1993, MNRAS, 261, 804

Higdon J.L., 1995, ApJ, 455, 524

Korchagin V., Vorobyov E.I., Mayya Y.D., 1999, ApJ, 522, 767

Kuzmin G., 1956, Astron. Zh, 33, 27

Little B., Carlberg R.G., 1991, MNRAS, 250, 161

Lynds R., Toomre A., 1976, ApJ, 209, 328

Marcum P.M., Appleton P.N., Higdon J.L., 1992, ApJ, 399, 57

Mihos J.C., Hernquist L., 1994, ApJ, 437, 611

Monaghan J.J., 1992, ARA\&A, 30, 543

Noguchi M., 1987, MNRAS, 228, 635

Noguchi M., 1988, A\&A, 203, 259 
Odewahn S.C., 1996, in Buta R., Crocker D.A., Elmegreen B.G., eds, ASP Conf. Ser. Vol. 91, Barred Galaxies. Astron. Soc. Pac., San Francisco, p. 30

Ostriker J.P., Peebles P.J.E., 1973, ApJ, 186, 467

Pèrez-Ramírez D., Knapen J.H., Peletier R.F., Laine S., Doyon R., Nadeau D., 2000, MNRAS, 317, 234

Plummer H.C., 1911, MNRAS, 71, 460

Pompea S.M., Rieke G.H., 1990, ApJ, 356, 416

Rix H.-W., 1993, PASP, 105, 999

Rix H.-W., Rieke M.J., 1993, ApJ, 418, 123

Seigar M.S., James P.A., 1998, MNRAS, 299, 672

Sellwood J.A., Sparke L.S., 1988, MNRAS, 231, 25

Sellwood J.A., Wilkinson A., 1993, Rep. Prog. Phys., 56, 173

Shlosman I., Noguchi M., 1993, ApJ, 414, 474

Shlosman I., Begelman M.C., Frank J., 1990, Nat, 345, 679

Spitzer L., 1942, ApJ, 95, 329

Steinmetz M., 1996, MNRAS, 278, 1005

Sugimoto D., Chikada Y., Makino J., Ito T., Ebisuzaki T., Umemura M., 1990, Nat, 345,33

Sundin M., Sundelius B., 1991, A\&A, 245, L5

Sundin M., Donner K.J., Sundelius B., 1993, A\&A, 280, 105

Tremaine S., Weinberg M.D., 1984, MNRAS, 209, 729

Toomre A., 1963, ApJ, 138, 385

Toomre A., 1964, ApJ, 139, 1217

Toomre A., 1978, in Longair M.S., Einasto J., eds, The Large Scale Structure of the Universe. D. Reidel, Dordrecht, p. 109

Toomre A., Toomre J., 1972, ApJ, 139, 179, 623

Weinberg M., 1985, MNRAS, 213, 451

Weinberg M., 1991, ApJ, 373, 391

Zaritsky D., Smith R., Frenk C., White S.D.M., 1993, ApJ, 405, 464

Zaritsky D., Smith R., Frenk C., White S.D.M., 1997, ApJ, 478, 39 


\title{
6. The regeneration of stellar bars by tidal interactions. Numerical simulations of fly-by encounters. ${ }^{1}$
}

\author{
I. Berentzen ${ }^{1,2}$, E. Athanassoula ${ }^{2}$, C.H. Heller ${ }^{3}$, and K.J. Fricke ${ }^{1}$ \\ ${ }^{1}$ Universitäts-Sternwarte, Geismarlandstraße 11, D-37083 Göttingen, Germany \\ ${ }^{2}$ Observatoire de Marseille, 2 Place Le Verrier, F-13248 Marseille Cedex 4, France \\ ${ }^{3}$ Georgia Southern University, Department of Physics, Statesboro, GA 30460, U.S.A.
}

\begin{abstract}
We study the regeneration of stellar bars triggered by a tidal interaction, using numerical simulations of either purely stellar or stellar+gas disc galaxies. We find that interactions which are sufficiently strong to regenerate the bar in the purely stellar models do not lead to a regeneration in the dissipative models, owing to the induced gas inflow in those models. In models in which the bar can be regenerated, we find a tight correlation between the strength and the pattern speed of the induced bar. This relation can be explained by a significant radial redistribution of angular momentum in the disc due to the interaction, similar to the processes and correlations found for isolated barred spirals. We furthermore show that the regenerated bars show the same dynamical properties as their isolated counterparts.
\end{abstract}

\subsection{Introduction}

Barred galaxies amount to more than one third of the catalogued disc galaxies in optical wavelengths (see, for example, de Vaucouleurs 1963) ant to about two thirds in the near-infrared (Eskridge et al. 2000). It has been established by now, that the life of a bar can be divided into several episodes: its formation, evolution, dissolution and maybe its regeneration (see, for example, the review by Friedli 1999).

Two mechanisms for the formation of bars in disc galaxies are being widely accepted at present. Bar formation can occur, as shown in many numerical simulations, spontaneously by a global instability in cold, rotationally supported stellar

\footnotetext{
${ }^{1}$ This manuscript has been accepted for publication in the Monthly Notices of the Royal Astronomical Society.
} 
discs (see, for example, Miller, Prendergast \& Quirk 1970; Hohl 1971; Ostriker \& Peebles 1973; Sellwood 1981; Athanassoula \& Sellwood 1986; etc.). The second mechanism, also confirmed in numerous $N$-body simulations, is the formation of tidally induced bars, triggered by interactions with neighbouring galaxies (see, for example, Byrd et al. 1986; Noguchi 1987, 1988; Gerin, Combes \& Athanassoula 1990; Barnes \& Hernquist 1991; Salo 1991; Miwa \& Noguchi 1998; etc.). To distinguish between the two bar forming mechanisms, Miwa \& Noguchi (1998) compared the properties of spontaneously formed and tidally induced bars by means of $N$-body simulations and argued that tidally induced bars are slow rotators, while the spontaneously formed bars have usually higher pattern speeds.

Athanassoula (2003) argued that the evolution of bars in isolated disc galaxies is driven by the redistribution of angular momentum. Since this is crucial for the work presented here, we will briefly summarise the main results. Disc galaxies strive to transfer angular momentum outwards (Lynden-Bell \& Kalnajs 1972). Disc material in the inner disc, being at resonance with the bar, emits angular momentum to resonant material in the outer disc, or in the spheroidal components like the halo and the bulge (Athanassoula 2002). The corotation radius of the bar roughly divides the regions of disc emitters from that of disc absorbers. The size and the pattern speed of the bar responds to the angular momentum exchange in a way as to keep an equilibrium between the emitters and absorbers. These recent results argue strongly that the strength and the pattern speed of the bar are determined by the amount of angular momentum exchanged. The bar's slowdown rate found in numerical simulations depends on the relative halo mass and on the velocity dispersion of both the disc and the halo. Furthermore, Athanassoula (2002) has shown that bar growth in the disc can be stimulated even in massive halos due to the destabilising influence of resonant stars in the halo.

Another factor influencing the evolution of bars is the interstellar medium in the disc. Owing to the gravitational torques of the bar, gas can be driven towards the centre of the galaxy and this is likely to be accompanied by central starbursts and formation/fueling of an active galactic nucleus (Shlosman, Begelman \& Frank 1989). Fully self-consistent numerical simulations including gas have shown that substantial gas inflow can weaken significantly, or even destroy, the bar (Friedli \& Benz 1993; Berentzen et al. 1998). The central mass concentrations and the times-scales found in these simulations, however, might be too high and too short, respectively, to be in agreement with the relatively high fraction of barred galaxies observed. Furthermore, this fraction seems to be independent of galaxy morphology, i.e. the same in early- and late-type disc galaxies, while early-types are known to have considerably less gas.

A regeneration of the stellar bar, i.e. a secondary episode of bar-formation during the lifetime of the disc, has been suggested as a possible mechanism to explain the observed number of barred galaxies. One scenario for this, as suggested by Sellwood \& Moore (1999), could be the accretion of gas-rich companion galaxies or freshly infalling gas, which, by adding colder material to the disc, may cool it 
sufficiently and allow the generation of a new bar. Another scenario suggested, and upon which we will focus in this paper, could be the regeneration of a previously dissolved or weakened bar by a tidal encounter with a neighbouring galaxy. In this work we study this regeneration process by means of fully self-consistent, 3D numerical simulations. The host galaxy, in which the first, i.e. spontaneously formed, bar has significantly weakened before the interaction, is tidally perturbed by a companion galaxy. Various orbits and masses have been considered for the companion, in order to cover a wide parameter space. We provide a comparison between pure stellar and two-component stars+gas models and describe the regeneration process and the dynamical properties of the tidally induced bars.

In $\$ 6.2$ we describe the numerical methods and the initial conditions of the galaxy models. The evolution and the dynamical properties of the isolated models with and without gas are described in $\S 6.3$ and $\S 6.4$, respectively. In $\S 6.5$ and $\S 6.6$ we present the results of the interacting models with and without gas, respectively. The results are then discussed in $§ 6.7$ and finally we give a summary in $§ 6.8$.

\subsection{Numerical methods and initial conditions}

\subsubsection{Methods}

To evolve both the collisionless component, representing the stars and the dark matter, and the dissipative component, representing the gas, we use an $N$-body algorithm, combined with a smoothed particle hydrodynamics (hereafter SPH) algorithm (see, for example, the review by Monaghan 1992). For this purpose we use the version of the hybrid $N$-body/SPH code of Heller (1991; see also Heller \& Shlosman 1994). The fully self-consistent 3D algorithm employs such features as a spatially varying smoothing length, a hierarchy of time-bins to approximate individual time-steps, a viscosity 'switch' to reduce the effects of viscous shear, and the special-purpose hardware GRAPE-3AF to compute the gravitational forces and neighbour interaction lists (Sugimoto et al. 1990; Steinmetz 1996).

\subsubsection{Initial conditions}

Our isolated model I0 is initially composed of a stellar and a gaseous disc, embedded in a spherical halo. The radial surface density of both the discs follows a truncated Kuzmin-Toomre (hereafter KT) profile (Kuzmin 1956; Toomre 1963) with a radial scalelength $a_{\mathrm{d}}$. Their vertical density profile follows the sech ${ }^{2}$ distribution of an isothermal sheet (Spitzer 1942). The halo is set up initially as a Plummer (hereafter Pl) sphere (Plummer 1911) with a radial scalelength $b_{\mathrm{h}}$, and is then allowed to relax in the gradually introduced potential of the frozen disc. For a detailed description of the initial model I0 see also Berentzen et al. (2003). The initial model parameters are summarized in Tab. 6.1. The columns from left to right 
Table 6.1: Initial model parameters.

\begin{tabular}{|c|c|c|c|c|c|c|}
\hline Component & Type & $N_{\mathrm{d}}$ & $\mathrm{M}_{\mathrm{d}}$ & $a_{\mathrm{d}}$ & $\mathrm{r}_{\text {cut }}$ & $\mathrm{z}_{0}$ \\
\hline \multicolumn{7}{|l|}{ Disc - I0 } \\
\hline stars & KT & 13500 & 0.54 & 1.0 & 5.0 & 0.20 \\
\hline gas & KT & 10000 & 0.16 & 1.0 & 5.0 & 0.05 \\
\hline \multicolumn{7}{|l|}{ Disc - I1 } \\
\hline stars & $\ldots$ & 17500 & 0.7 & $\ldots$ & $\ldots$ & $\cdots$ \\
\hline Component & Type & $N_{\mathrm{h}}$ & $\mathrm{M}_{\mathrm{h}}$ & $b_{\mathrm{h}}$ & $\mathrm{r}_{\text {cut }}$ & \\
\hline \multicolumn{7}{|l|}{ Halo } \\
\hline stars & $\mathrm{Pl}$ & 32500 & 1.30 & 5.0 & 10.0 & $\ldots$ \\
\hline Component & Type & $N_{\mathrm{c}}$ & $\mathrm{M}_{\mathrm{c}}$ & & & \\
\hline \multicolumn{7}{|l|}{ Companion } \\
\hline $\mathrm{C} 1$ & $\mathrm{pt}$ & 1 & 2.0 & $\ldots$ & $\ldots$ & $\ldots$ \\
\hline $\mathrm{C} 2$ & $\mathrm{pt}$ & 1 & 4.0 & $\ldots$ & $\ldots$ & $\ldots$ \\
\hline $\mathrm{C} 3$ & $\mathrm{pt}$ & 1 & 6.0 & $\ldots$ & $\ldots$ & $\ldots$ \\
\hline $\mathrm{C} 4$ & $\mathrm{pt}$ & 1 & 8.0 & $\ldots$ & $\ldots$ & $\cdots$ \\
\hline $\mathrm{C} 5$ & $\mathrm{pt}$ & 1 & 0.66 & $\ldots$ & $\ldots$ & $\cdots$ \\
\hline C6 & $\mathrm{pt}$ & 1 & 1.0 & $\ldots$ & $\ldots$ & $\ldots$ \\
\hline
\end{tabular}

give the component, the type of density profile, the particle number, the total mass, the radial scalelengths $a_{\mathrm{d}}$ and $b_{\mathrm{h}}$, the cut-off radius $r_{\text {cut }}$, and the vertical scaleheight $z_{0}$ of each component. Our isolated model I1 differs from model I0 in that about one third of the gas particles have been replaced with stellar particles, i.e. with keeping their positions and assigning new masses, keeping the total disc mass the same as in model I0. The remaining gas particles have been completely removed from the disc. We then assign velocities to the new stellar particles according to the mean velocity distribution of the old underlying stellar disc. Both host galaxies, I0 and I1, are evolved in isolation first. The disc of I0 is constructed so as to be globally unstable to non-axisymmetric perturbations and form a large-scale bar. Owing to induced gas-inflow, the stellar bar in model I0 weakens significantly during its evolution, but is present in both models before the companion galaxy is introduced.

The companion galaxy in the interaction simulations is represented by a smoothed point mass (hereafter pt) for simplicity, applying a Plummer softening for the force calculation. Since the GRAPE-3 hardware can only handle a fixed softening length, we apply the same softening for all particles, including the companion. The mass ratio $M_{\text {host }}: M_{\text {comp }}$, where $M_{\text {host }}$ and $M_{\text {comp }}$ are the total mass of the host and of the companion galaxy, respectively, has been varied between $1: 1$ to $1: 4$. In addition a 
few simulations have been run with less massive companions. For the simulations presented in this paper we confine ourselves to planar fly-by encounters, i.e. the orbits of the host and the companion galaxy are unbound and lie in the equatorial plane of the host. The initial conditions have been chosen such as to provide a strong perturbation to the disc. The orbit of the companion galaxy is prograde with respect to the rotation in the host disc and has been chosen to be either parabolic or hyperbolic, with different pericentric separations $R_{\text {peri }}$. The pericentric position has been chosen such that at time of pericentre (hereafter $t_{\text {peri }}$ ) the major axis of the relatively weak bar, which has formed/evolved in the hosts disc before the encounter, points towards the companion. The times of pericentric separation have been chosen to be $t_{\text {peri }}=270$ and 500 for all simulations with host galaxy I0 and I1, respectively. The initial orbital parameters at $t_{\text {peri }}$ have been calculated from the solution of the corresponding two-body problem, either for a given orbital eccentricity $e$, or for a pericentric angular frequency of the companion

$$
\omega_{\text {peri }}=v_{\text {peri }} / R_{\text {peri }},
$$

where $v_{\text {peri }}$ is the velocity of the companion at pericentre. To obtain the initial centre of mass positions and velocities of the host and of the companion galaxy, we integrate their orbits backward in time, starting from the pericentric time $t_{\text {peri }}$, until the distance $\Delta R$ between host and companion is roughly 10 times the cut-off radius of the initial halo. During this backwards orbit integration the particles of the host are frozen with respect to each other. Owing to the limited spatial range of the GRAPE-3 hardware, the interaction models have been calculated in the inertial frame of the host galaxy and the force of the companion has partly been calculated by direct summation on the front-end, i.e. without using the GRAPE hardware.

\subsubsection{Units}

The adopted units for mass, distance, and time are $M=6 \times 10^{10} \mathrm{M}_{\odot}, \mathrm{R}=3 \mathrm{kpc}$ and $\tau=10^{7} \mathrm{yr}$, respectively, for which the gravitational constant $\mathrm{G}$ is unity. The dynamical time is $\tau_{\text {dyn }} \equiv\left(r_{1 / 2}^{3} / \mathrm{G} M_{1 / 2}\right)^{1 / 2}=4.8 \times 10^{7} \mathrm{yr}$, where $M_{1 / 2}$ is half the total mass of the host and $r_{1 / 2}$ is its half-mass radius, which is, after relaxation of the halo, approximately $8.5 \mathrm{kpc}$ or in terms of the disc scalelength, $2.8 a_{\mathrm{d}}$. The initial stellar disc rotation period in these units then corresponds to $t_{\text {rot }} \equiv 2 \pi \tau_{\text {dyn }} \approx 3 \times 10^{8} \mathrm{yr}$. A fixed gravitational softening length of $\epsilon=0.375 \mathrm{kpc}$ is used for all particles. An isothermal equation of state is used for the gas with a sound speed of $v_{\mathrm{s}}=12 \mathrm{~km}$ $\mathrm{s}^{-1}$. The corresponding thermal gas temperature is $10^{4} \mathrm{~K}$.

\subsection{Isolated model with gas - model I0}

As described in the previous section, the isolated galaxy model I0 is composed of a stellar (collisionless) and a gaseous (dissipative) disc component, embedded in 
o

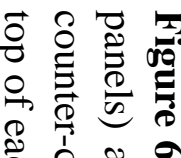

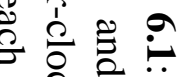

象

获芯

き 官导

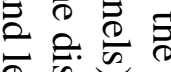
$\stackrel{2}{2} \cong$ च ڤ ڤ คิ कृ ฉ क $\cong \stackrel{0}{\circ}$ ڤ 放 吉年 झ

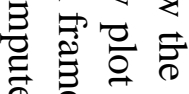
- $ᄋ \stackrel{\circ}{\circ}$


है 듕 站 产.

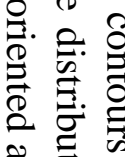
๊ 宁 궁 鬲 - 2 . \&

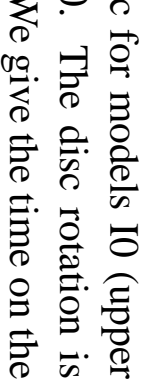
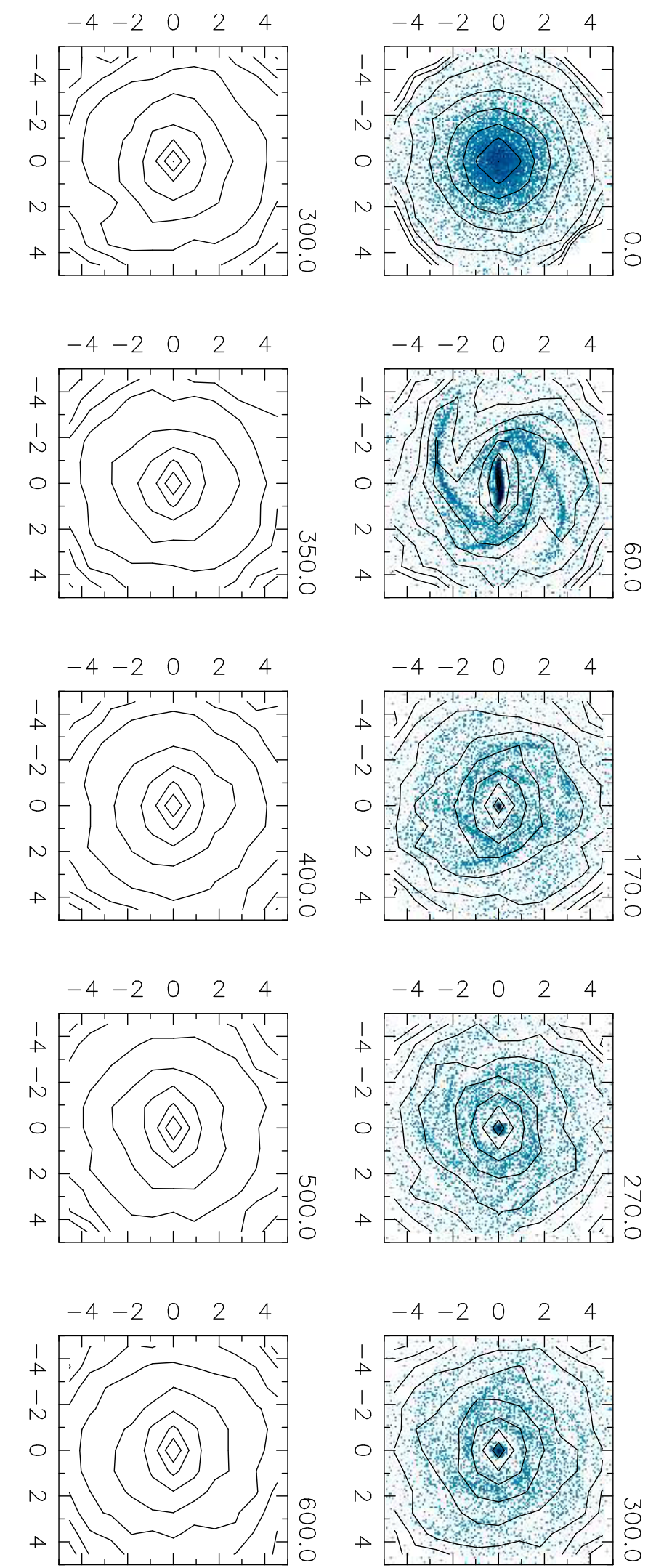
a live halo. The morphological evolution of the disc is shown in Fig. 6.1 (upper panels). The stellar disc has been chosen to be initially bar unstable and forms a large-scale bar within roughly $2 t_{\text {rot }}$. The stellar bar reaches its maximum strength at about $t=60$ (see Fig. 6.2). The bar strength, here defined by the normalized $m=2$ Fourier component of the stellar disc mass distribution, has been measured inside a cylindrical radius of $R=1.25$ and about one scaleheight of the disc plane. Owing to the torque of the bar, most of the gas in the bar region is driven towards the centre of the galaxy, accumulating in a nuclear and circumnuclear disc. At the end of the run the mass of the gaseous nuclear disc represents some 18 per cent of the total mass within a spherical radius of $1.0 \mathrm{kpc}$. As a result of the growing central mass concentration the stellar bar weakens significantly, and the disc settles down in a quasi-stable state at about $t \approx 120$. Some gas also accumulates near the end of the weak stellar bar, forming an oval ring close to the position of the inner ultra-harmonic resonance (hereafter UHR) at $R=2.25 \mathrm{kpc}$. The pattern speed of the bar (hereafter $\Omega_{\mathrm{p}}$ ) first increases due to the short burst of gas inflow and thereafter reaches a constant rate of about $\Omega_{\mathrm{p}}=0.3 \tau^{-1}$, or $29.3 \mathrm{~km} \mathrm{~s}^{-1} \mathrm{kpc}^{-1}$. The evolution of model I0 is followed in total up to $t=300$, i.e. about 10 disc rotations. A more detailed description of the evolution of this model and its dynamical properties is given in Berentzen et al. (2003).

To identify the presence and the location of the main planar resonances in the disc, we apply both the linear (epicyclic) approximation and non-linear methods. We therefore calculate the gravitational potential in the $z=0$ plane of the disc on a Cartesian grid and symmetrise it with a four-fold symmetry with respect to the main axes of the bar. From the potential we then derive the azimuthally averaged circular and epicyclic frequency $\Omega_{\mathrm{c}}(R)$ and $\kappa(R)$, respectively. The standard linear resonance condition is given by

$$
\Omega_{\mathrm{p}}=\Omega_{\mathrm{c}}+\frac{l}{m} \kappa
$$

with integers $l$ and $m$. For a strict definition of the inner Lindblad resonances (hereafter ILRs; with $l=-1$ and $m=2$ ) three extensions to the linear definition have been proposed (see Athanassoula 2003 for a discussion), which are not fully compatible to each other and sometimes in contradiction. Throughout this work we apply the orbital structure definition for the ILRs, i.e. by saying that an ILR exists if and only if the $x_{2}$ and $x_{3}$ orbit families exist. The ILRs have been confirmed in this case by calculating the surfaces of section (hereafter SOS). The SOS have been constructed by integrating orbits of a given Jacobian energy (hereafter $E_{J}$; see also Sec. 6.4) in the equatorial plane of the disc and marking the points in the $(y, \dot{y})$ plane each time the orbits cross the line $x=0$ with $\dot{x}<0$ (see, for example, Binney \& Tremaine 1987). At the end of the run $(t=300)$ the corotation radius (hereafter CR; i.e., the radius at which $\left.\Omega_{\mathrm{c}}=\Omega_{\mathrm{p}}\right)$ in this model is at about $6.15 \mathrm{kpc}\left(E_{\mathrm{J}} \approx-0.91\right)$, and the ILRs are located at about $0.47 \mathrm{kpc}$ (inner ILR; $E_{\mathrm{J}}=-1.58$ ) and $2.1 \mathrm{kpc}$ (outer ILR; $\left.E_{\mathrm{J}}=-1.10\right)$, respectively. 


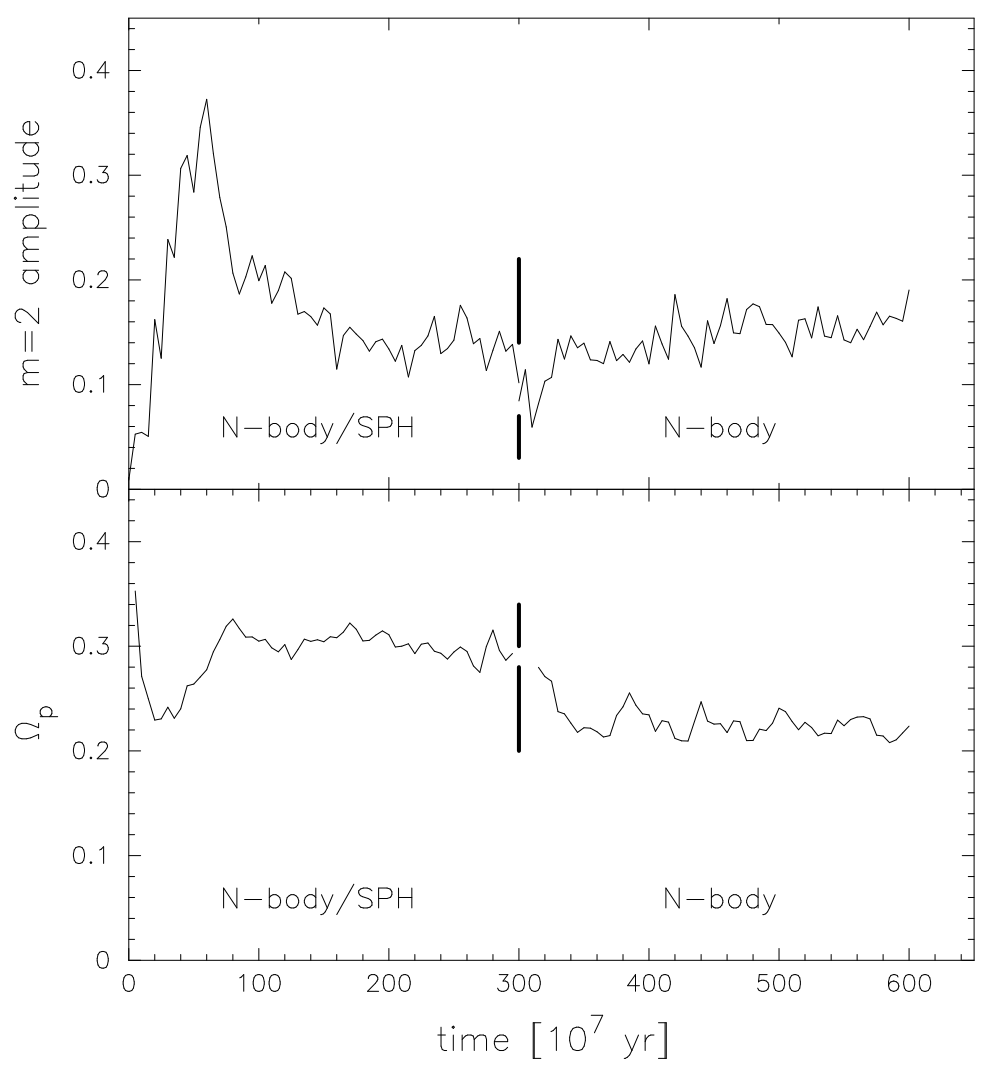

Figure 6.2: Evolution of the bar strength (upper panel) and pattern speed (lower panel) for models I0 (stars+gas) and I1 (stars). The transition between the two models is marked by two vertical dashes $(t=300)$.

\subsection{Isolated model without gas - model I1}

We study the regeneration of the bar by interactions of two different types of host galaxies - with and without gas in the disc - which, nevertheless, have similar dynamical properties. We therefore construct our purely stellar model I1 by replacing the gas particles in model I0 with stellar particles, as described in Sec. 6.2.2. This has been accomplished for the last snapshot of model I0 at $t=300$, i.e. at the end of the run, when the amplitude of the initial bar has very considerably decreased and the disc is in a quasi-stable state. After replacing the gaseous particles with stellar ones we follow the evolution of the new model I1 for another $\Delta t=300$. The morphological evolution of the stellar disc is shown in Fig. 6.1 (lower panels), and in Fig. 6.2 we show the evolution of the bar strength and of the pattern speed. The bar strength first decreases abruptly, owing to the newly added stellar particles, which follow initially the distribution of the gas. The phase angle of the $m=2$ Fourier component of the latter is shifted with respect to that of the old underlying disc 


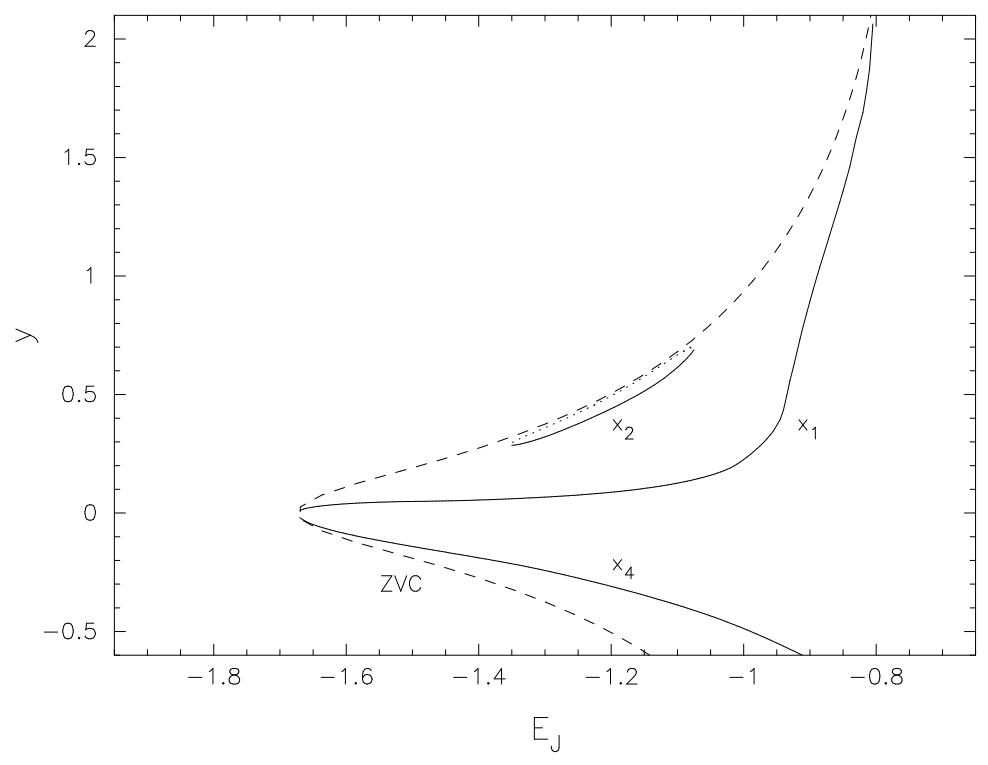

Figure 6.3: Characteristic diagram of the isolated model I1 at the end of the run. We show the characteristic curves of the main orbit families (full lines) and the zero velocity curve (ZVC; dashed line).

and therefore tends to decrease the normalized $m=2$ amplitude of the combined discs. The velocities, which are assigned to the new stellar disc component, follow the velocity distribution of the old underlying stellar disc. Therefore the stellar disc of model I1 is initially slightly out of virial equilibrium, since the dispersion of the stellar particles is higher than that of the gas particles. After some $\Delta t=30$, however, the disc settles to a new equilibrium and the bar strength increases again, reaching its previous value and remaining constant after the short relaxation phase. Owing to the decreasing central mass concentration of the new stellar component, the pattern speed of the bar slightly decreases first, but remains roughly constant after the relaxation phase at about $\Omega_{\mathrm{p}}=0.22 \tau^{-1}$, or $21.5 \mathrm{~km} \mathrm{~s}^{-1} \mathrm{kpc}^{-1}$, till the end of the run.

To gain some insight in the orbital structure of the disc of model I1, we locate the main planar periodic orbits in a frozen potential that rotates with the bar. General information on orbits in barred potentials can be found, e.g., in Contopoulos \& Grosbøl (1989). The gravitational potential has been calculated the same way as described in the previous section, but is time-averaged over roughly one bar rotation. For simplicity, we restrict ourselves to planar orbits which are bi-symmetric with respect to the bar and close after one orbit around the centre in the frame of reference corotating with the bar. The results are then displayed in terms of a characteristic diagram, where for each orbit we plot its Jacobi integral $E_{\mathrm{J}}$ with respect to the $y$ intercept value with the $x=0$ plane. The Jacobi integral is a conserved quantity 
Table 6.2: Notation of the interaction models.

\begin{tabular}{|c|c|c|c|c|}
\hline \multirow[b]{2}{*}{$R_{\text {peri }}$} & \multicolumn{3}{|c|}{$\omega_{\text {peri }}$} & \multirow{2}{*}{$\begin{array}{l}\text { para- } \\
\text { bolic }\end{array}$} \\
\hline & 0.3 & 0.6 & 0.9 & \\
\hline 15.0 & $\mathrm{a}$ & $\mathrm{b}$ & $\mathrm{c}$ & $p$ \\
\hline 10.0 & $\mathrm{~d}$ & $\mathrm{e}$ & $\mathrm{f}$ & $\mathrm{p}_{1}$ \\
\hline 5.0 & $\mathrm{~g}$ & $\mathrm{~h}$ & $\mathrm{i}$ & $\mathrm{p}_{2}$ \\
\hline
\end{tabular}

along any given orbit in the rotating frame, and can be thought of as an effective energy (see, for example, Binney \& Tremaine 1987). In the characteristic diagram the orbits form curves of families. In Fig. 6.3 we show the characteristic diagram of the main orbit families, using the notation of Contopoulos \& Papayannopoulos (1980), in model I1 at the end of the run $(t=600)$. The dashed curve is the zerovelocity curve (hereafter $\mathrm{ZVC}$ ), which delineates the accessible region in the plane based on energy considerations. The family labelled $x_{1}$ consists of orbits that are elongated along the bar and predominately gives the bar its structure. Orbits of the $x_{2}$-family are elongated perpendicular to the bar. Their presence is indicative of an ILR (or more than one) in the non-linear regime. The $x_{4}$ orbits are retrograde and slightly elongated perpendicular to the bar. The corotation radius in model I1 is at $E_{\mathrm{J}}=-0.79$, or approximately $7.98 \mathrm{kpc}$. The $x_{2}$-orbits range in energy from $E_{\mathrm{J}}=-1.35$ to -1.07 , with semi-major axes ranging from 0.86 to $2.1 \mathrm{kpc}$.

\subsection{Interacting models with gas}

In this section we describe the simulations in which the gas-rich host galaxy I0 is perturbed by encounters with companions of different mass. The time of pericentric separation has been chosen to be $t_{\text {peri }}=270$ for all runs with the host galaxy I0. In the first set of simulations the companions are initially set up to follow prograde, parabolic orbits with a pericentric separation of $R_{\text {peri }}=15$, which is just outside the halo of the host galaxy. When referring to a specific model, we will hereafter use a notation like 'I0 C4 p', where 'I0' is the name of the host galaxy, 'C4' is the name of the companion (see Tab. 6.1), and 'p' (parabolic) denotes the type of orbit, the latter following the notation given in Tab. 6.2. In Tab. 6.5 we summarise the basic parameters and properties of both the isolated and the interaction models. The first and the second column give the name of the host and of the companion galaxy, respectively, and the third column gives the orbit of the companion (see Tab. 6.2). In the fourth column we give the time when the companion is included in the simulation, resulting from the initial backward orbit integration (see Sec. 6.2.2). The fifth to seventh column give the pericentric separation and frequency, and the eccentricity 


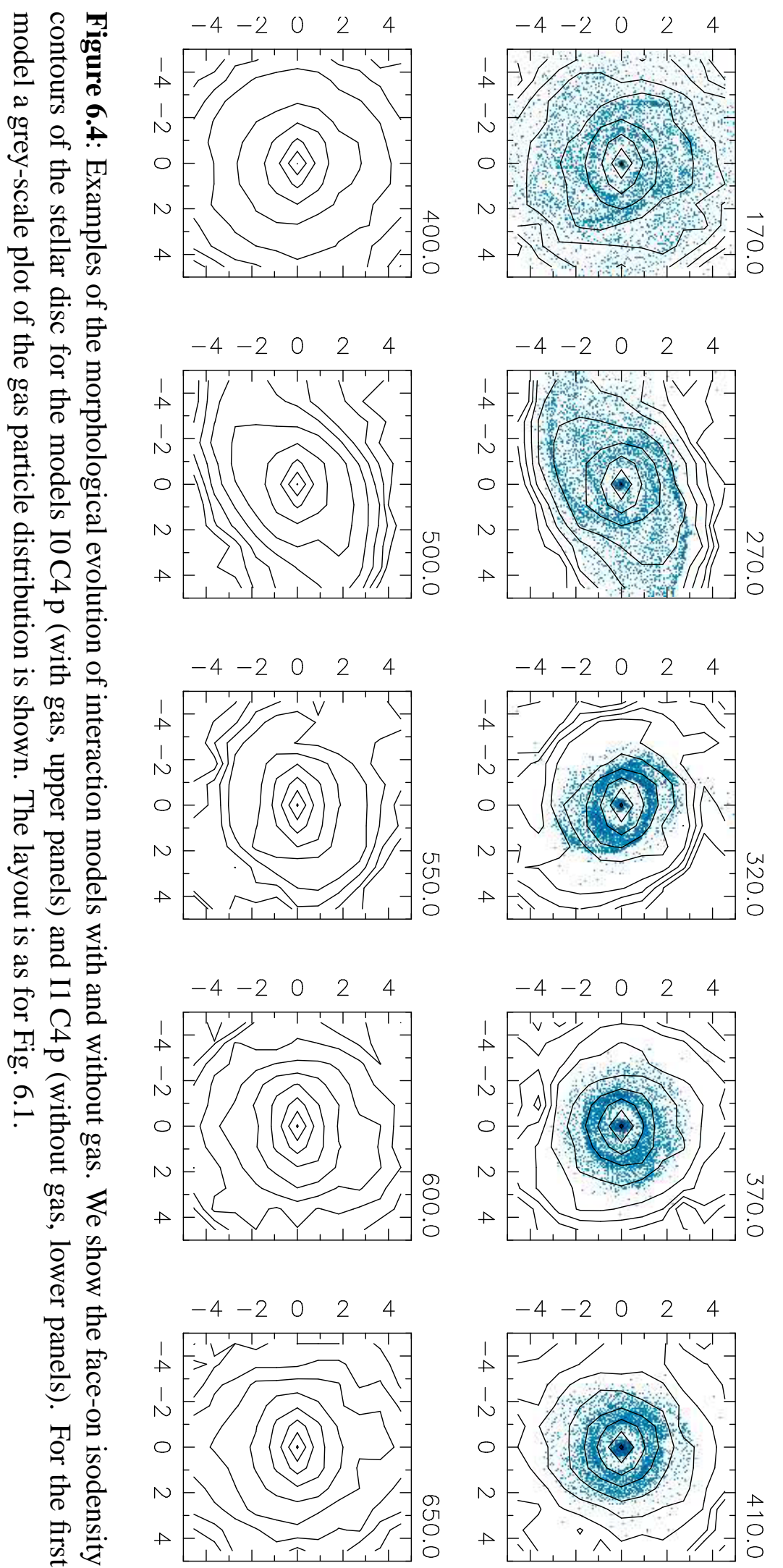



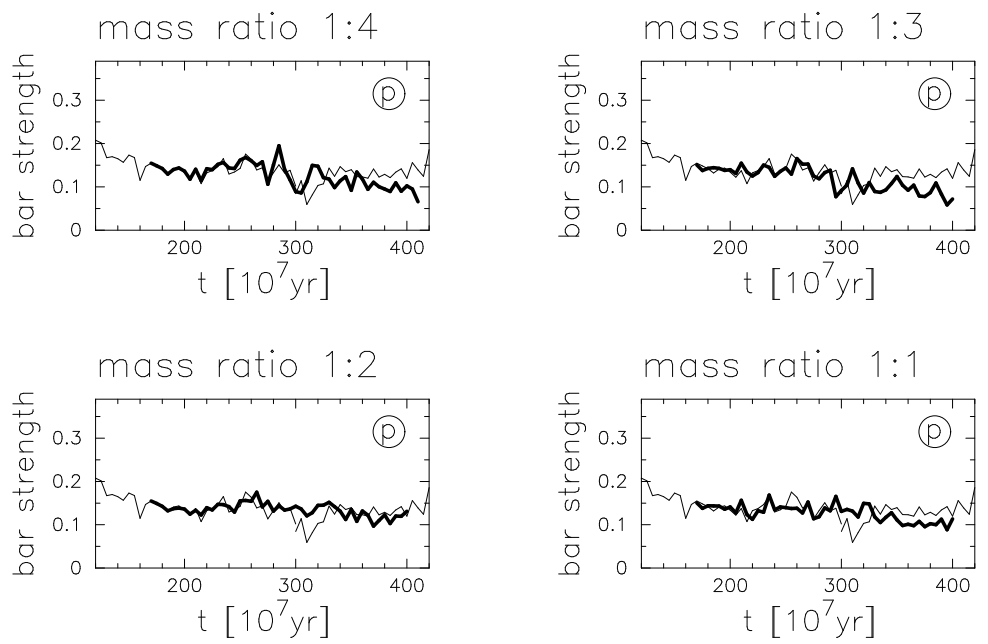

Figure 6.5: Evolution of the bar strength as a function of time for prograde, parabolic encounters with host galaxy I0 (thick line). The pericentric separation is $R_{\text {peri }}=15$ and the mass ratio $M_{\text {host }}: M_{\text {comp }}$ is given on top of each frame. The type of the companions orbit is given in the upper right-hand corner of each frame. For comparison we also show the bar strength of the isolated model (thin line).

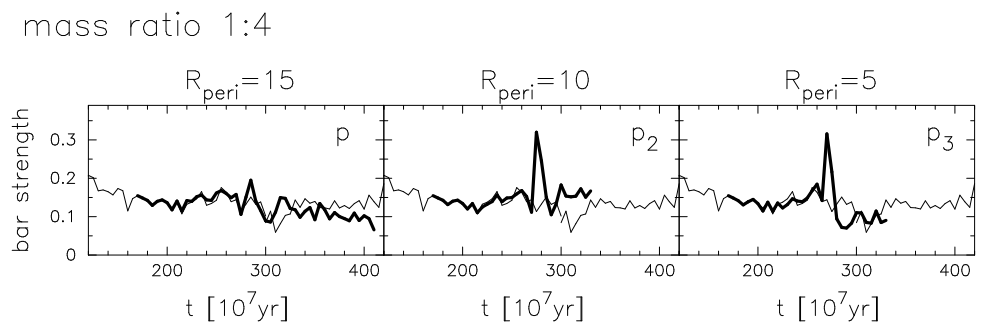

Figure 6.6: Same as Fig. 6.5, but for encounters with a fixed mass ratio of $1: 4$ and pericentric separations of $15(\mathrm{p}), 10\left(\mathrm{p}_{2}\right)$ and $5\left(\mathrm{p}_{3}\right)$, respectively.

of the orbit, respectively. In the eighth and ninth column we give the strength and pattern speed of the bar, respectively, after the interaction. The tenth and eleventh columns give the ratio of bar strength and pattern speed to the corresponding values of the isolated model. In the penultimate column we give the interaction strength parameter as defined in Sec. 6.6.1 and in the last column the corotation radius at the end of each run, determined from the linear analysis.

In Fig. 6.4 (upper panels) we show an example of the morphological evolution of the disc during an encounter, in this case for model I0 C4 p. The bar strength as a function of time for the models with different massive companions is shown in Fig. 6.5. We find that the encounters in this set of simulations are not sufficiently strong to regenerate the stellar bar in the disc, even for a mass ratio of $1: 4$, i.e. even 


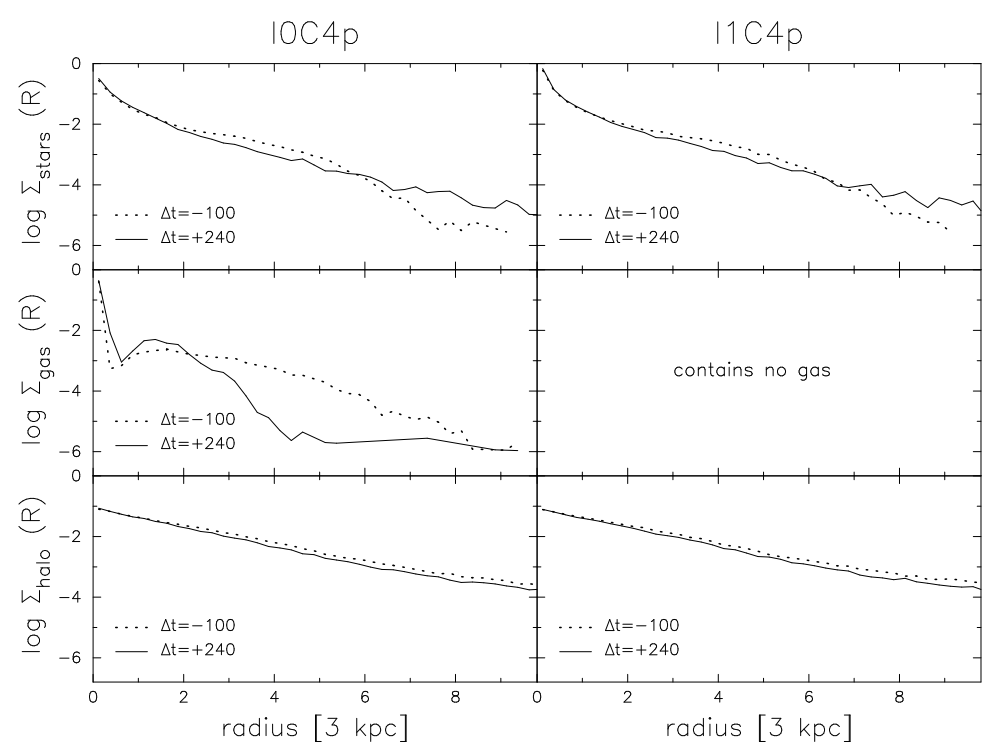

Figure 6.7: Logarithmic surface density as a function of radius for the stellar and gaseous disc and of the halo (from top to bottom, respectively). We show $\log \Sigma(R)$ for models I0 C4 p (left-hand panels) and I1 C4p (right-hand panels). The dotted and full lines represent times before $(\Delta t=-100)$ and after $(\Delta t=240)$ the interaction, respectively.

with companion $\mathrm{C} 4$. In order to increase the strength of the tidal perturbation on the host galaxy, we run two additional simulations with companion $\mathrm{C} 4$, in which we now choose pericentric separations of $R_{\text {peri }}=10$ and 5 . The orbits of the companion are again chosen to be prograde and parabolic. The bar strength for these models is shown in Fig. 6.6. The peaks immediately following $t_{\text {peri }}$ result from the time when the transient tidal arms contribute to the $m=2$ power in the inner disc region, in which the bar amplitude is measured, but disappear as soon as the tidal arm features have dissolved. Even with these close encounters, it is not possible to regenerate the stellar bar in the disc of the gas-rich host galaxy.

In order to facilitate comparison with the purely stellar simulations described in the next section, we present here a more detailed analysis of the interaction simulations with the gas-rich host galaxy I0. As can be seen in Fig. 6.4 already, the interaction apparently leads to a significant redistribution of both the stellar and the gaseous material in the disc. This becomes more evident in Fig. 6.7, in which we show the logarithmic radial surface density of the disc and the halo for model I0 C4 p at times before and after the interaction. While the stellar density remains basically constant within a radius of approximately $R<2.0$, i.e. roughly inside corotation in the corresponding isolated model, there is some net inflow of gas from the outer disc. The inflowing gas first piles up at a radius of about $R \approx 1.25$, which 


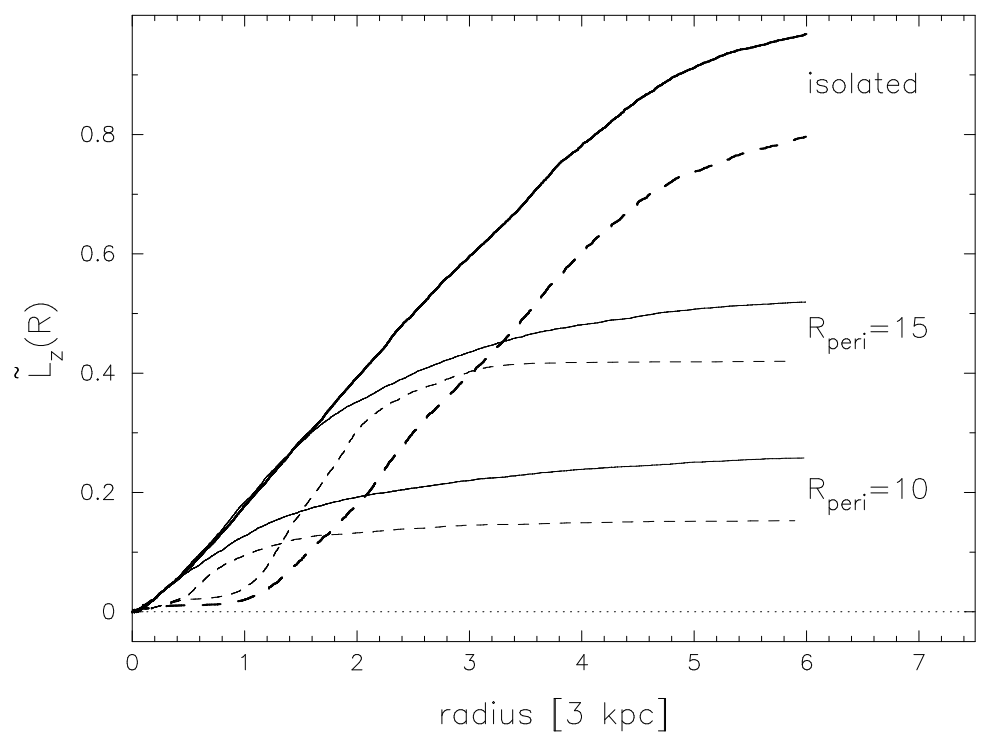

Figure 6.8: Specific cumulative angular momentum $\tilde{L}_{z}$ as a function of radius in the stellar disc at the end of the run of the isolated model I0 (with gas) and of the interaction models with a mass ratio of $1: 4$ and different pericentric separations. The full and dashed lines show the results for the stellar and gaseous disc, respectively.

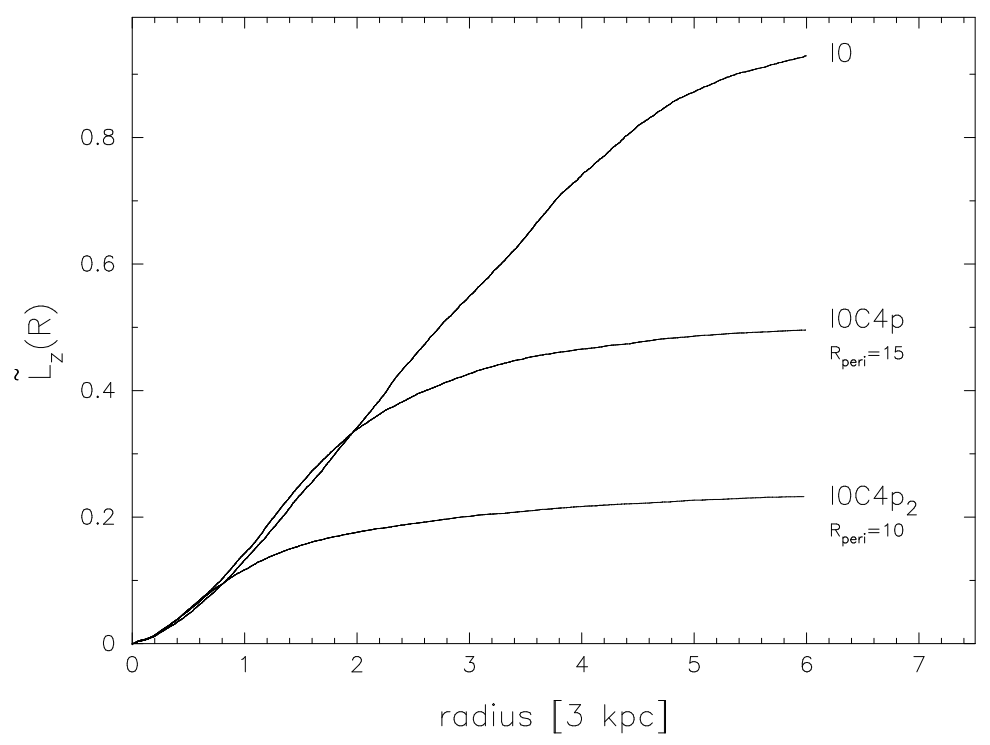

Figure 6.9: Same as Fig. 6.8, but for the combined disc (stars+gas).

is close to the position of the gaseous ring, and then flows gradually towards the central disc region, accumulating in the nuclear disc. At the end of the run, the gas 
mass within 0.5 length units has increased by about 0.5 per cent of the total host mass, or some 6 per cent of the total gas mass, while the gas mass within 2 length units increased by roughly 1 per cent of the total mass, or about 12 per cent of the total gas mass. Some fraction of both the stellar and the gaseous material in the outer disc moves further out, contributing to the transient tidal arms and/or getting stripped off by the companion. The halo material in the inner region does not show any net radial redistribution, but we find some expansion in the outer parts, where halo material is also stripped off by the companion.

We measure also the specific cumulative angular momentum (hereafter $\tilde{L}_{z}$ ) as a function of radius for both the stellar and the gaseous disc, given by

$$
\tilde{L}_{z}(R)=\left(\sum_{R_{i}<R} l_{z, i}\right) \times\left(\sum_{R_{i}<R} m_{i}\right)^{-1}
$$

where $R_{i}, m_{i}$ and $l_{z, i}$ are the planar radius, the mass and the angular momentum, respectively, of the $i$-th disc particle. As an example we show in Fig. 6.8 the results of simulations with companion $\mathrm{C} 4$ and different pericentric separations. As can be seen in this plot, the interaction removes angular momentum from the stellar disc at all radii shown. We do not find any indication that the corotation radius of the bar or of the companion separates regions which gain from regions which lose angular momentum. More angular momentum is removed from the stellar disc for the closer pericentric separation. The gaseous disc shows a different behaviour. The gas in the inner disc gains angular momentum compared to the isolated model, within roughly $R=3.0$ and 1.7 for pericentric separations of $R_{\text {peri }}=15$ and 10 , respectively, and loses angular momentum outside those radii.

In Fig. 6.9 we show the specific cumulative angular momentum as a function of radius in the combined disc (stars+gas). We find that the disc in the interaction models I0 C4 p and I0 C4 $\mathrm{p}_{2}$ does not lose, or even gains, angular momentum within a radius of $R \approx 2.0$ and $R \approx 1.0$, respectively, as compared to the isolated model $\mathrm{I} 0$.

\subsection{Interacting models without gas}

In this section we describe the set of simulations with the purely stellar model I1 as the host galaxy. We start with simulations having similar orbits for the interaction as the ones described in the previous section, i.e. prograde and parabolic encounters using different companions (see Tab. 6.5). As shown in Fig. 6.10, in which we plot the bar strength as a function of time, simulations with mass ratios of $1: 3$ and $1: 4$ are, contrary to the dissipative models, sufficiently strong to regenerate the stellar bar in the disc, and the strength of the induced bar increases with the mass of the companion. In Fig. 6.11 we show as an example the characteristic diagram of the host galaxy of the interaction model I1 C4 $\mathrm{p}$ at time $t=640$, i.e. the end of the run, in which the stellar bar has been successfully regenerated by the interaction. The 


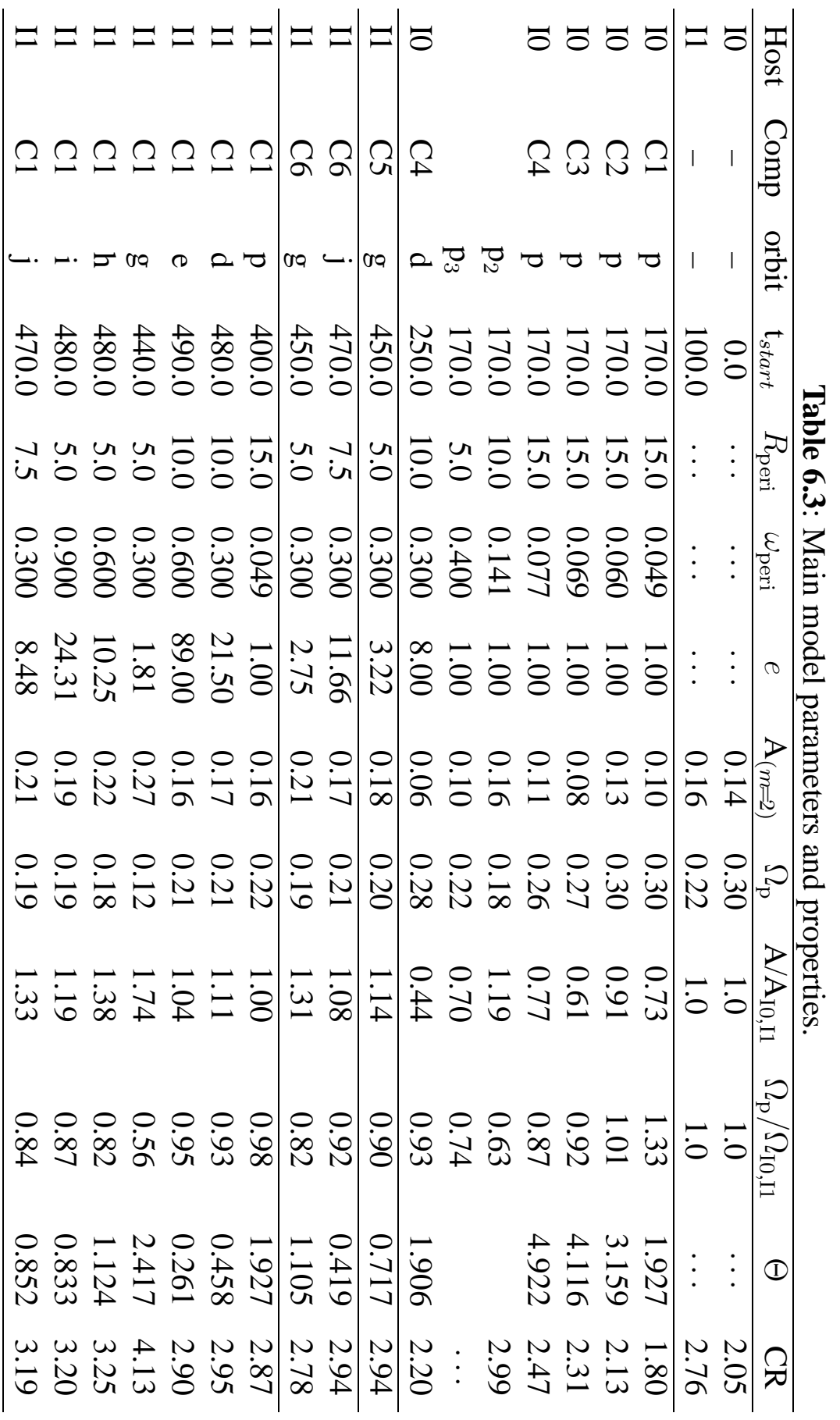




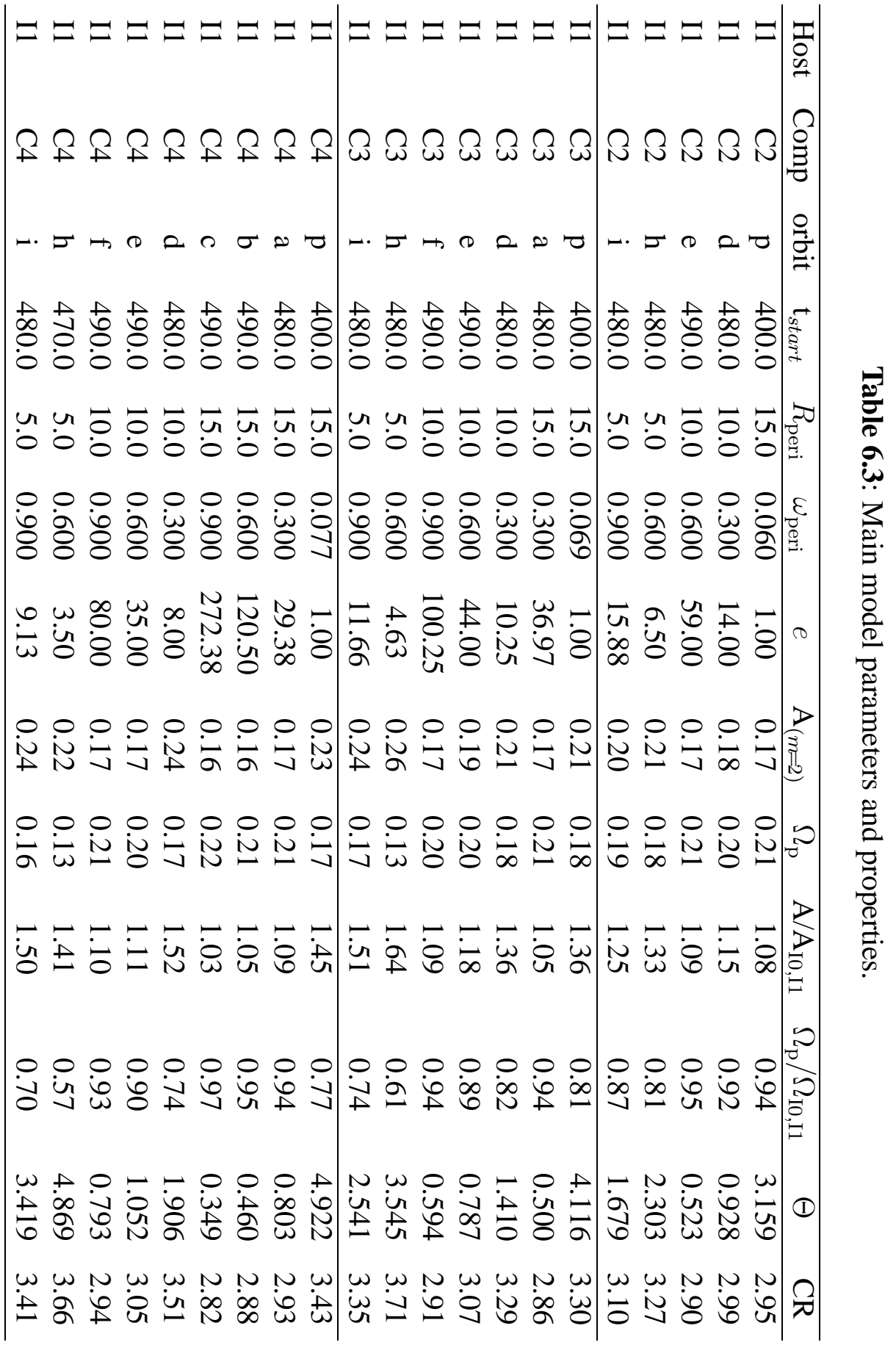



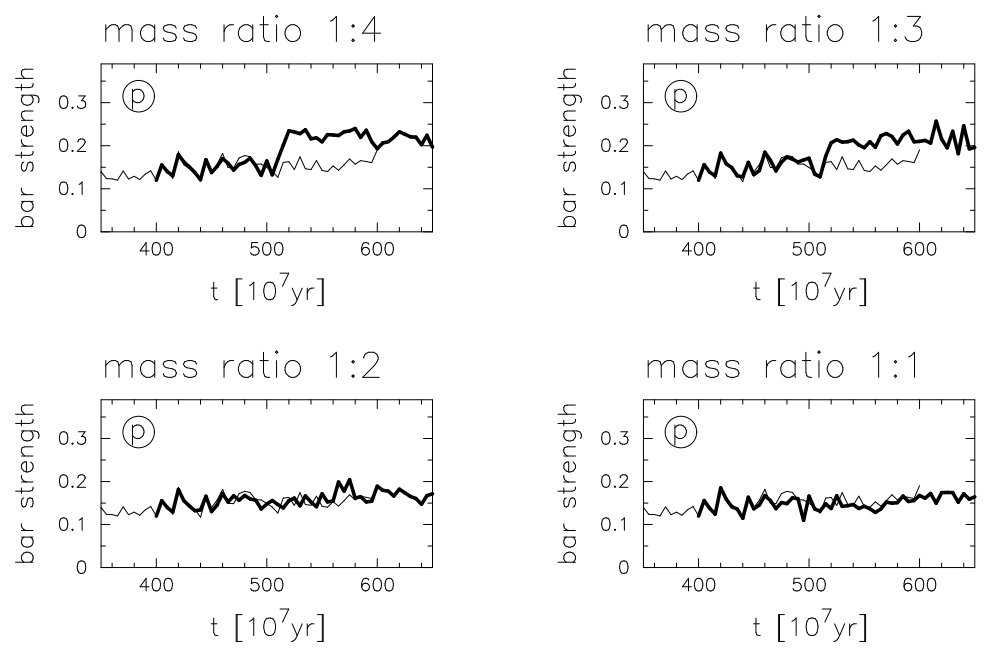

Figure 6.10: Bar strength as a function of time for the prograde, parabolic encounters with the purely stellar model I1 (thick line). The layout is as in Fig. 6.5.

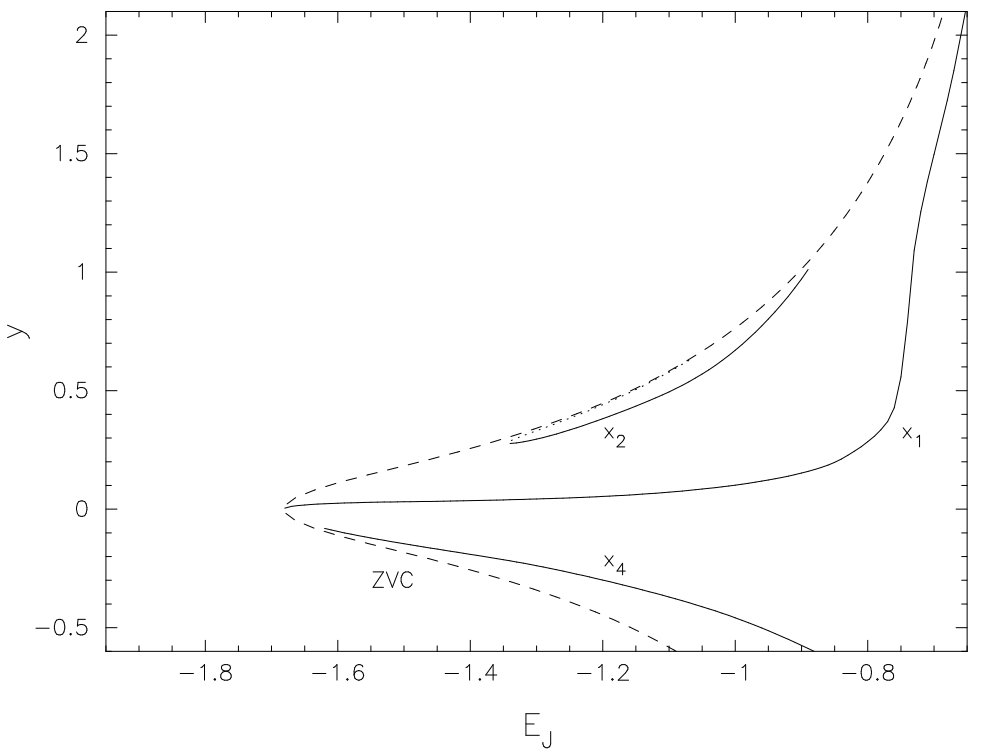

Figure 6.11: Characteristic diagram of model I1 C4 p after the interaction. The layout is as in Fig. 6.3.

orbit analysis has been performed following the description given in Sec. 6.4. The corotation radius in this model is at $E_{\mathrm{J}}=-0.63$, or about $10.3 \mathrm{kpc}$. The $x_{2}$ orbits range in energy from $E_{\mathrm{J}}=-1.34$ to -0.88 , with semi-major axes ranging from 0.83 to $3.04 \mathrm{kpc}$.

Motivated by these results, we now explore a much wider - though still far from 


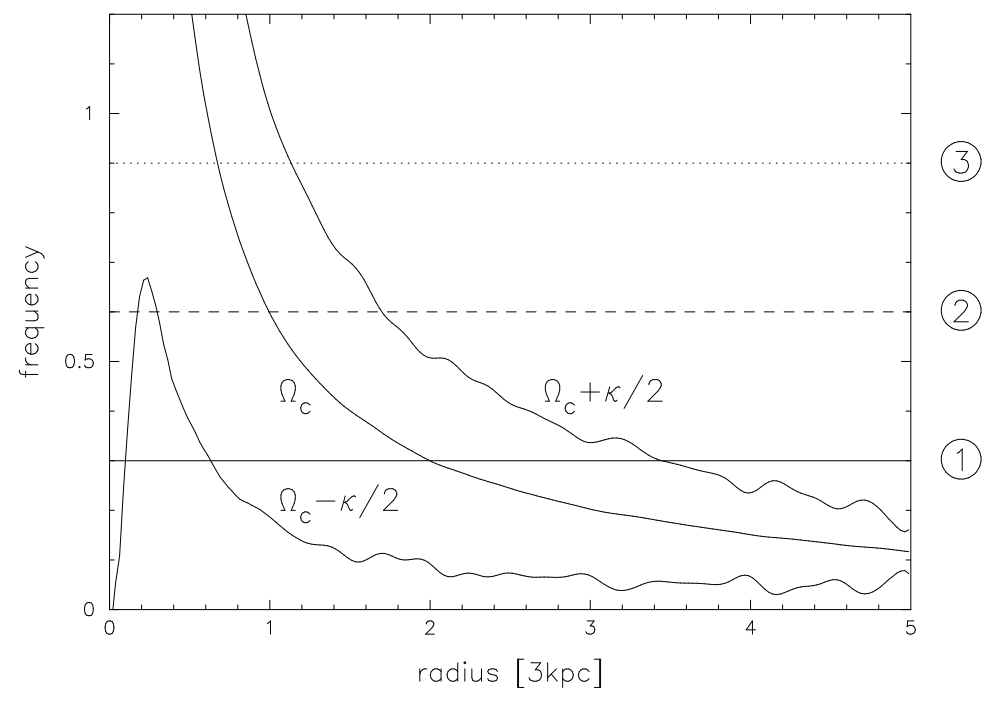

Figure 6.12: Basic frequencies of the isolated model I1 $(t=500)$ as a function of radius, obtained with the linear axisymmetric definition, using the epicyclic approximation. The horizontal lines mark the angular frequency $\omega_{\text {peri }}$ of the companion at the time of pericentric separation.

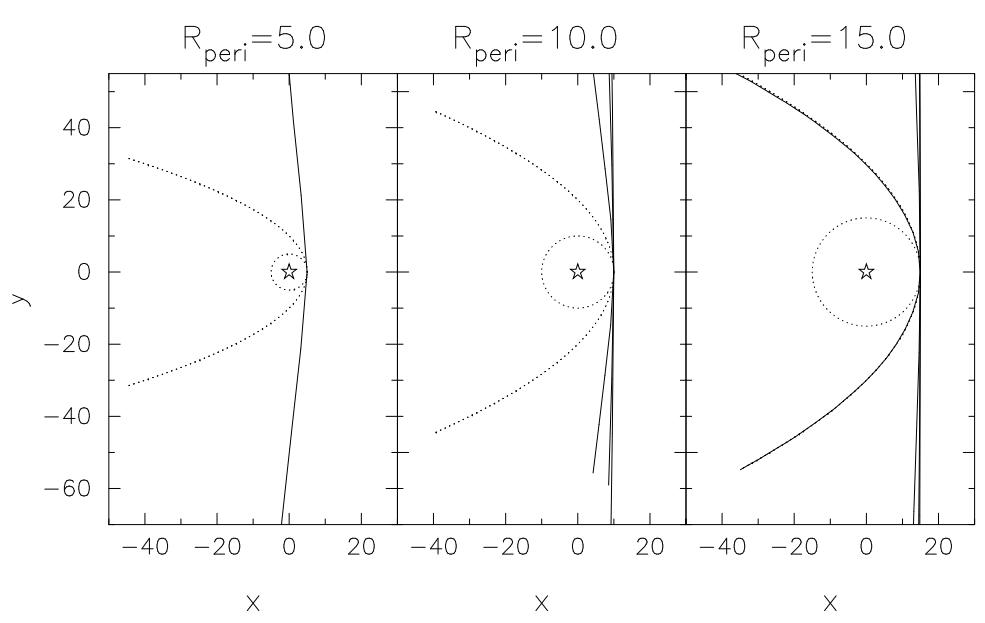

Figure 6.13: Different trajectories of the companion galaxy $\mathrm{C} 4$. We show the parabolic and hyperbolic orbits (full lines) from simulations with different pericentric separations, as given on top of each panel. The corresponding circular and parabolic orbits are indicated by dotted lines. The host galaxy is marked by a star in each panel.

complete - parameter range, in order to determine the initial conditions necessary (or sufficient) to regenerate the stellar bar in the disc. We therefore run a set of 
simulations, in which we vary the orbit of the companion, the pericentric separation $R_{\text {peri }}$ and the mass $M_{\mathrm{C}}$, respectively. The simulations and their basic initial parameters are summarized in Tab. 6.5. From the solution of the corresponding two-body problem the eccentricity $e$ of the companions orbit has been determined by its angular frequency $\omega_{\text {peri }}$ at pericentre, and we restrict ourselves in this work to unbound orbits only. We choose $\omega_{\text {peri }}$ such that a prescribed number of inner Lindblad resonances is present in the disc, i.e. that certain orbits in the disc are in resonance with the companion, when passing $R_{\text {peri. }}{ }^{2}$ Since the stellar bar in the unperturbed host galaxy is very weak, we apply the linear approximation, as described in Sec. 6.3, in order to determine the resonances in the disc. The resonance diagram for the isolated model I1 is shown in Fig. 6.12, and the frequencies chosen for the companion are:
1. $\omega_{\text {peri }}=0.3 \quad$ (two ILRs)
2. $\omega_{\text {peri }}=0.6 \quad$ (one ILR)
3. $\omega_{\text {peri }}=0.9 \quad$ (no ILR) .

The mass of the companion for these simulations has been varied, as before, from $1: 1$ to $1: 4$, and we have a few additional runs with even lower mass ratios, namely $1: \frac{1}{2}$ (C6) and $1: \frac{1}{3}$ (C5). A complete list of all simulations and of their main parameters is given in Tab. 6.5. Some of the companion orbits are illustrated in Fig. 6.13 for different eccentricities and pericentric separations.

In Fig. 6.14 we show the bar strength as a function of time for this set of interaction models. It is striking that - in contrast to the models with gas - the interaction is sufficiently strong to regenerate the bar in about half our models. As can also be seen from the plot, there is a general trend, that the strength of the induced bar increases with

- increasing mass $M_{\mathrm{C}}$ of the companion,

- decreasing pericentric separation $R_{\text {peri }}$,

- decreasing pericentric frequency $\omega_{\text {peri }}$.

This is what one might generally expect, since the gravitational force of the companion is proportional to its mass, the tidal force decreases with $R^{-3}$, and the time integrated force of the companion on the host galaxy becomes stronger for slower passages.

Furthermore, after the bar has formed in the disc, we find that the amplitude of the bar stays constant with time. We have checked the life-time of the regenerated bar by running model $\mathrm{I} 1 \mathrm{C} 4 \mathrm{~d}$, which shows a significant increase in bar strength after the interaction, for roughly $\Delta t=4 \times 10^{10} \mathrm{yr}$ in total. Using a linear-fitting

\footnotetext{
${ }^{2}$ We point out that the angular frequency of the companion, and therefore also the location of the corresponding resonances in the disc, changes with time.
} 

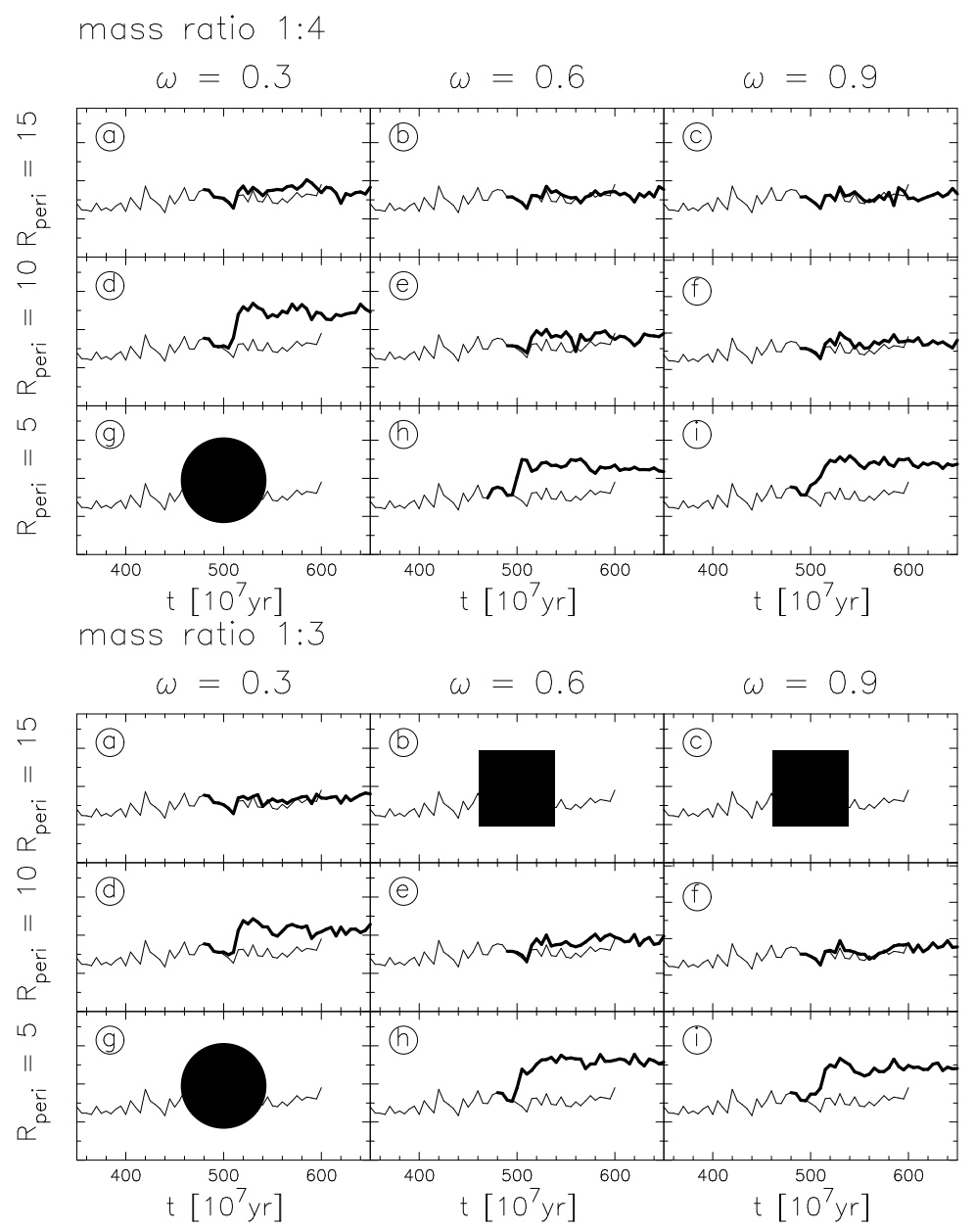

Figure 6.14: Bar strength as a function of time for some of the pure stellar models. The four frames show the models with mass ratios of $1: 1,1: 2,1: 3$ and $1: 4$, respectively. The filled squares mark models which have not been run, because other runs allow us to deduce that a bar cannot form. Models in which the companion would be on a bound elliptical orbit are marked by a filled circle.

we find a decay-rate of the bar of roughly $9 \times 10^{-12} \mathrm{yr}$. With this the bar will drop to half its amplitude after approximately $\Delta t=5 \times 10^{10} \mathrm{yr}$. After one Hubble time (with $H_{0}^{-1}=1.3 \times 10^{10} \mathrm{yr}$ ) the bar amplitude would have dropped only by 12 per cent. A fraction of this decrease could be introduced by the relatively low number of particles used in the simulations, so that in fact there could be (almost) no sign of a decay for bars formed in interactions. These results strongly argue for the fact that bars formed by the interaction are long-living and by no means transient phenomena. 

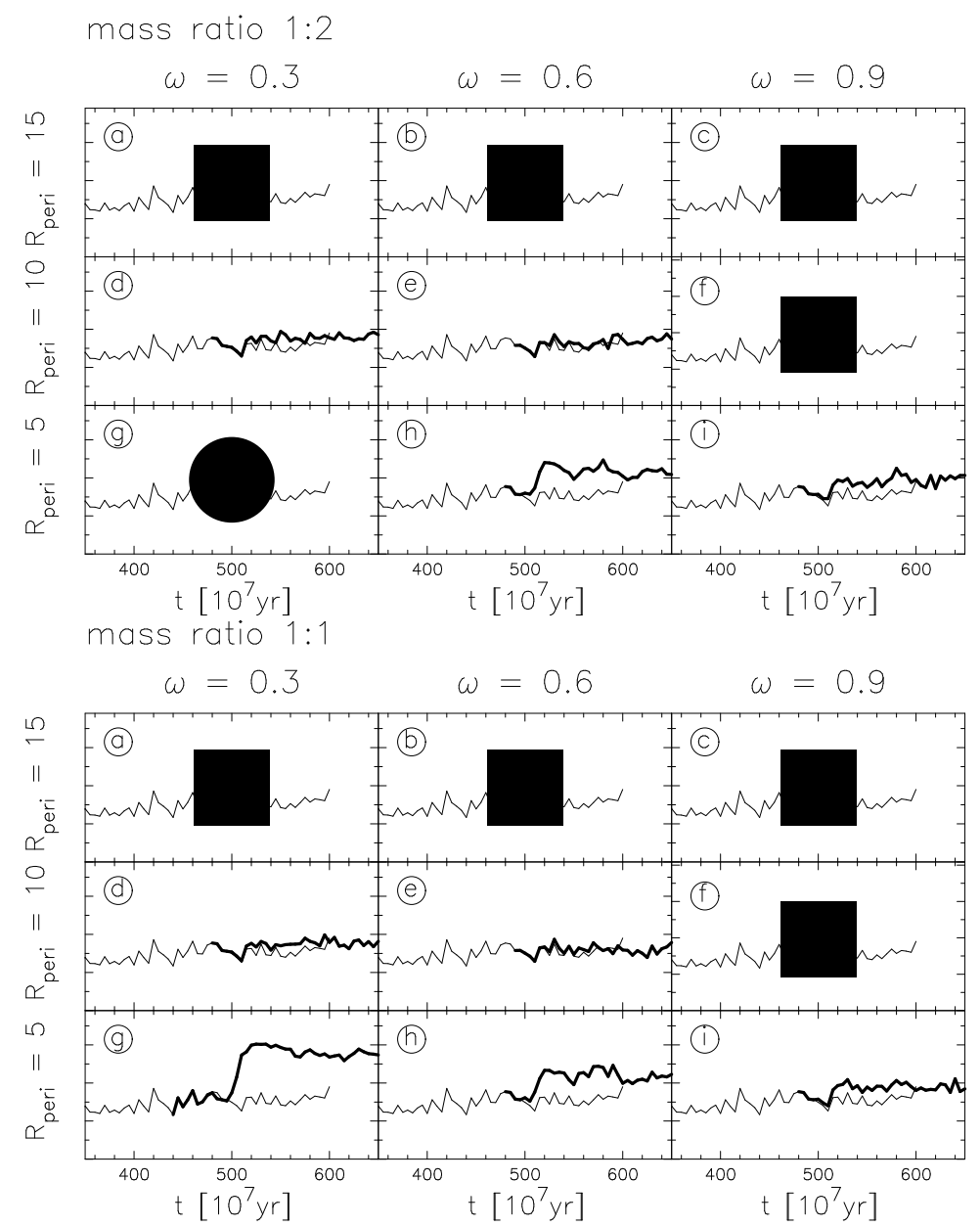

Figure 6.14: continued

\subsubsection{Dynamical properties of the bars}

To quantify the correlations described in the previous section, we define a parameter $\Theta$, which allows us to evaluate the interaction strength:

$$
\Theta \equiv\left\langle\frac{f_{\text {comp }}}{f_{\text {gal }}}\right\rangle=\int \frac{f_{\text {comp }}}{f_{\text {gal }}} \mathrm{d} t
$$

where $f_{\text {gal }}$ and $f_{\text {comp }}$ are the mean radial forces, exerted from the host I1 and the companion galaxy, respectively, averaged over the equatorial plane of the host galaxy. In practice we calculate them on a radial equally-spaced polar grid with maximum radius $R=4.8$ and then take a density weighted average. The integral in Eq. 6.4 is carried out from $t=-250$ to $t=250$, with $t=0$ corresponding to 


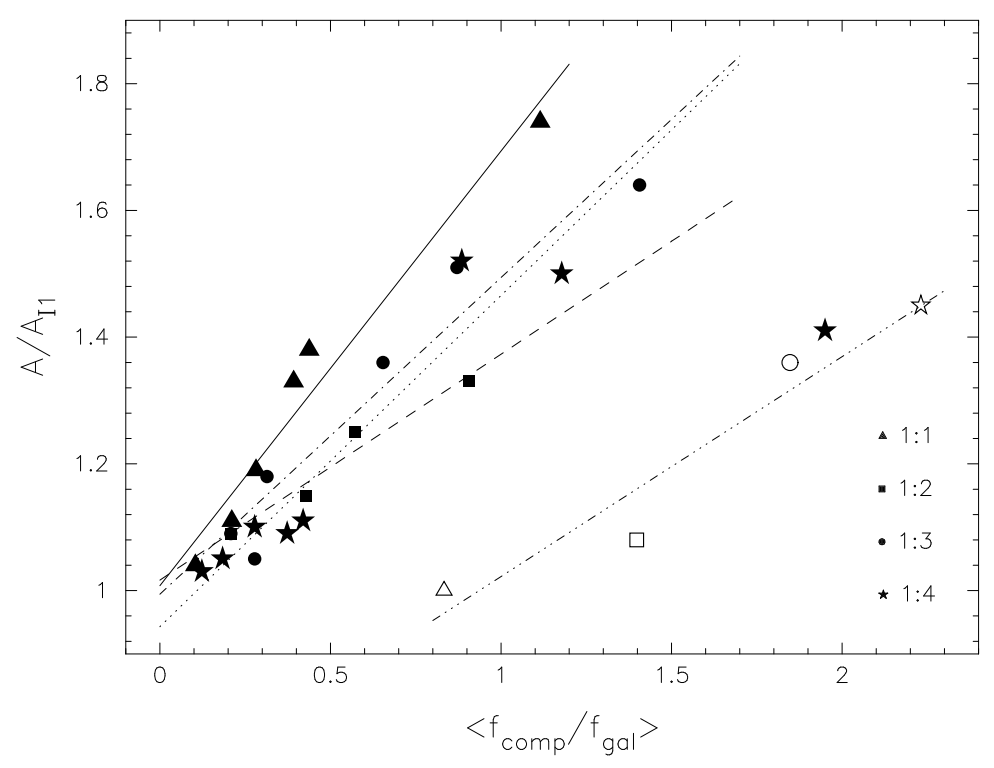

Figure 6.15: Normalized bar strength $A / A_{I 1}$ versus the interaction strength parameter $\Theta$. We show the results of the purely stellar simulations and the corresponding linear fits for mass ratios of $1: 1$ (bullets, full line), $1: 2$ (squares, dashed line), $1: 3$ (triangles, dotted-dashed line) and 1:4 (stars, dotted line). The simulations with parabolic orbits are shown with open symbols and are not taken into account for the linear fits.

$t_{\text {peri }}$, in order to guarantee an adequate convergence of $\Theta$ in all models. For these force calculations both galaxies are approximated by point masses for simplicity. The results for $\Theta$ are given in the penultimate column of Tab. 6.5. We also calculate the normalized bar strength $A / A_{\mathrm{I} 1}$, where $A$ and $A_{\mathrm{I} 1}$ are the bar strength in the interaction model and in the isolated model, respectively. By plotting the normalized bar strength versus the interaction strength parameter $\Theta$ (Fig. 6.15), we find a roughly linear correlation between the two quantities for each companion $\mathrm{C} i$, with $i=1 \ldots 4$. One of the $1: 4$ models ( $\mathrm{I} 1 \mathrm{C} 4 \mathrm{~h}$ ) is systematically off-set in all plots of this kind (i.e., Figs 6.15, 6.17 and 6.19), but always lies within the $2 \sigma$ confidence limit. The models with parabolic encounters are all off-set from the relationships found, but seem to be correlated themselves linearly.

Plotting the bar strength of the different models in a diagram showing the logarithmic orbital eccentricity $e$ versus the angular frequency $\omega_{\text {peri }}$ (see Fig. 6.16), we find that models with orbits of roughly $\log e>1.2$ are not sufficiently strong to regenerate the bar in the purely stellar models. A larger sample of simulations has to be run, however, to confirm the existence of such a limiting eccentricity. If such a limit really exists, it would give a necessary, but not sufficient condition for tidally induced bar formation for our purely stellar model. 


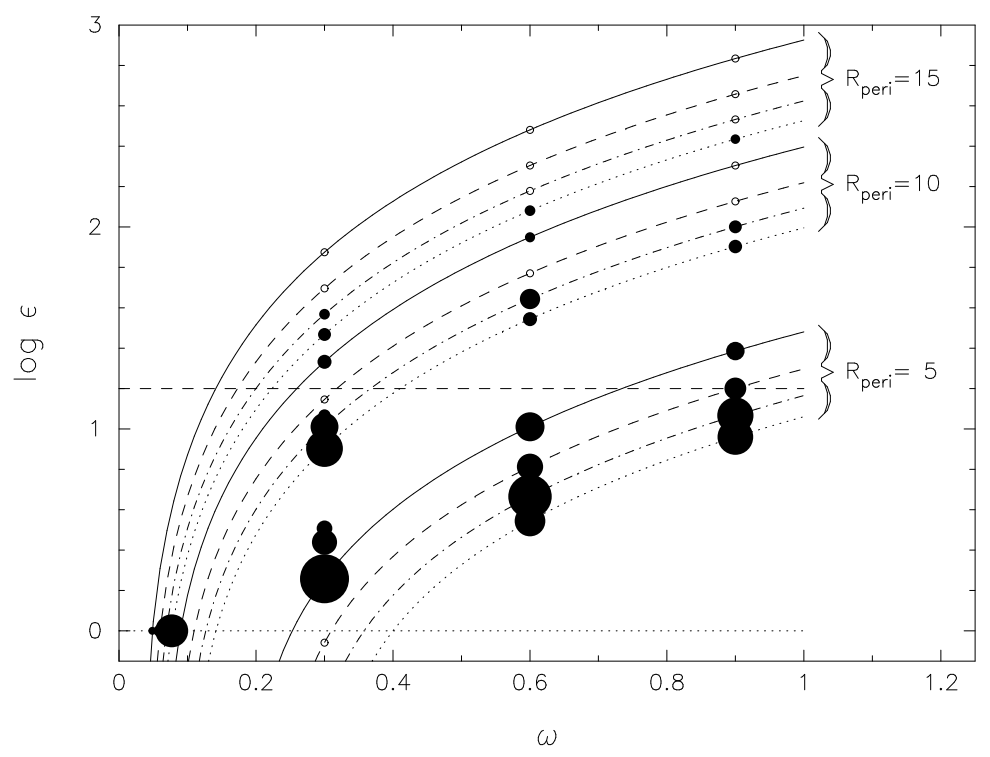

Figure 6.16: Logarithmic orbital eccentricity versus the angular frequency of the companion at pericentre time. For each pericentric separation the curves obtained from the corresponding two-body problem are plotted for different mass ratios: $1: 1$ (full line), 1:2 (dashed), 1:3 (dotted-dashed) and 1:4 (dotted line). The filled circles mark the different simulations and their size is proportional to the bar strength at the end of each run. The two circles which do not lie on a line are simulations with mass ratios of $1: 0.5$ and $1: 0.33$. The limit of $\epsilon$, as explained in the text, is marked by the horizontal dashed line.

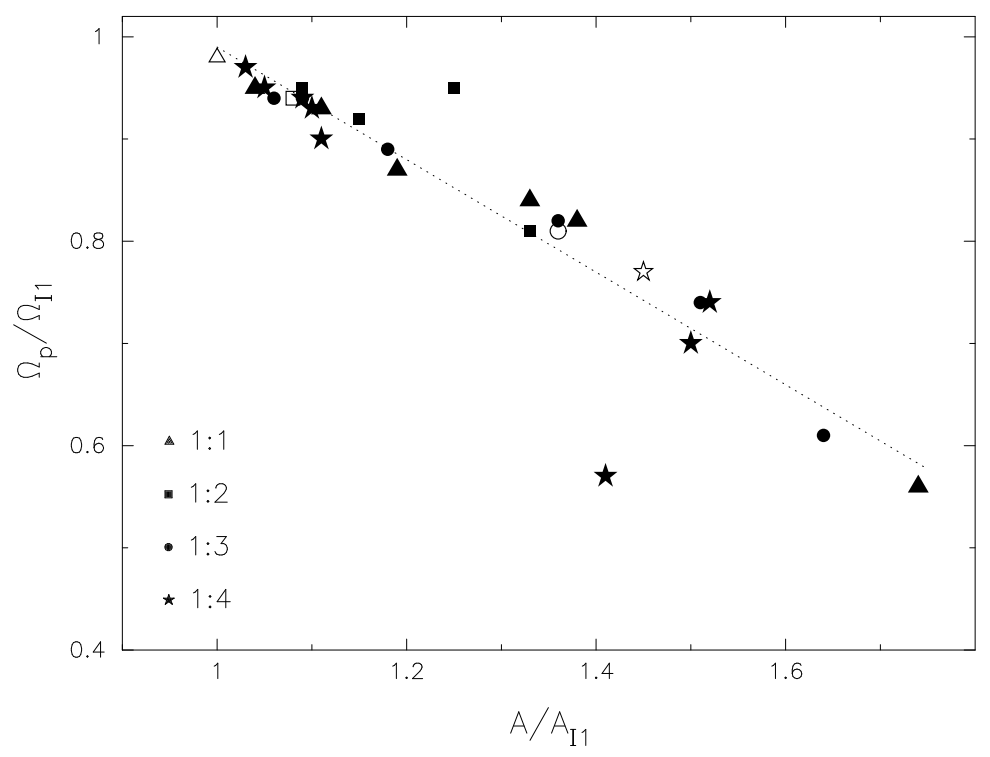

Figure 6.17: Normalized pattern speed $\Omega_{\mathrm{p}} / \Omega_{\mathrm{I} 1}$ versus normalized bar strength $A / A_{\mathrm{I} 1}$ of the purely stellar interaction models. Symbols are as in Fig. 6.15. 


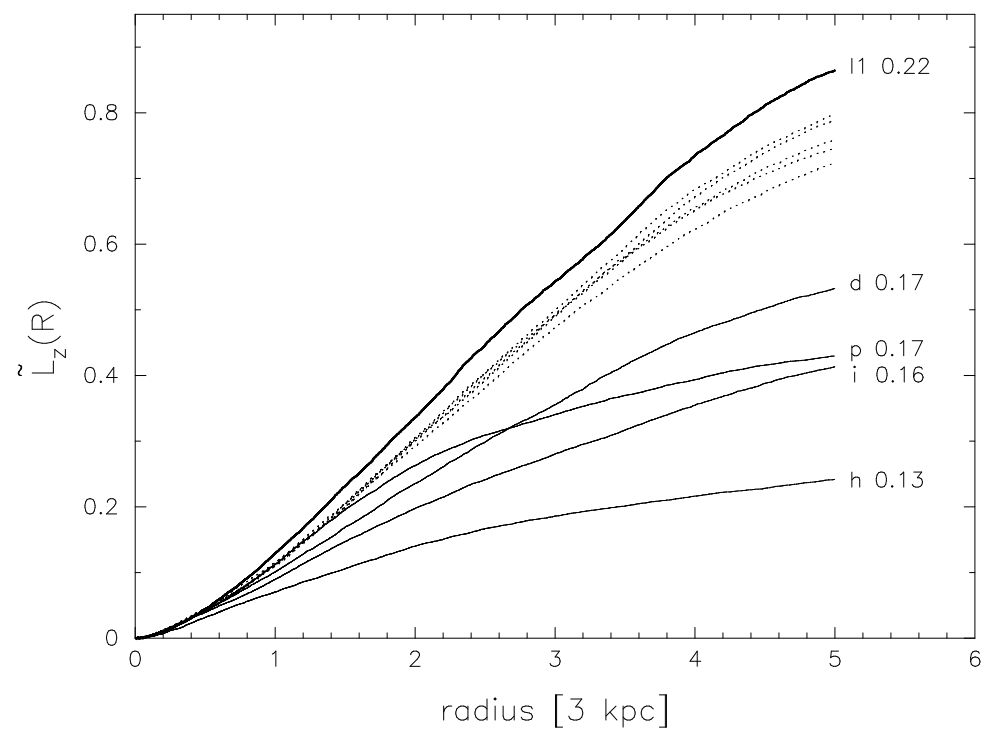

Figure 6.18: Specific cumulative angular momentum of the stellar disc for the isolated model I1 (thick line) and different interaction models I1 C4 (thin lines). The dotted lines indicate models in which no or only a weak increase of bar strength has been found after the interaction. Models in which the bar has been regenerated (full lines) are labelled (with the orbit type as given in Tab. 6.5) and the corresponding bar pattern speed is given.

Angular momentum exchange in isolated galaxies leads to correlations between the bar pattern speed and strength, as well as between the bar strength and the angular momentum gained by the spheroid, which, in many cases, is a measure of the angular momentum exchanged (Athanassoula 2003). We will now test whether such correlations can be found in our regenerated bars. We indeed find a tight correlation between the strength and the pattern speed of the regenerated bars (Fig. 6.17). The stronger the bar gets after the interaction, the lower becomes its pattern speed, in good agreement with Athanassoula (2003). We further find that the pattern speed in the interaction models is always lower than in the corresponding isolated case.

The passage of the companion again is accompanied by a redistribution of disc angular momentum. The angular momentum exchange between the different disc regions (as defined in the previous section) is similar to that in Fig. 6.8. In Fig. 6.18 we show the radial distribution of the specific cumulative angular momentum $\tilde{L}_{z}(R)$ for a set of simulations with companion $\mathrm{C} 4$ in comparison with the isolated model I1. For the runs with lower mass companions, we find similar results. It is noticeable that the regeneration of the stellar bar has been feasible only in those models which show a significant change in $\tilde{L}_{z}$. Actually we find a tight correlation between the loss of angular momentum $\Delta L_{z}$ in the disc measured inside the initial disc cut-off 


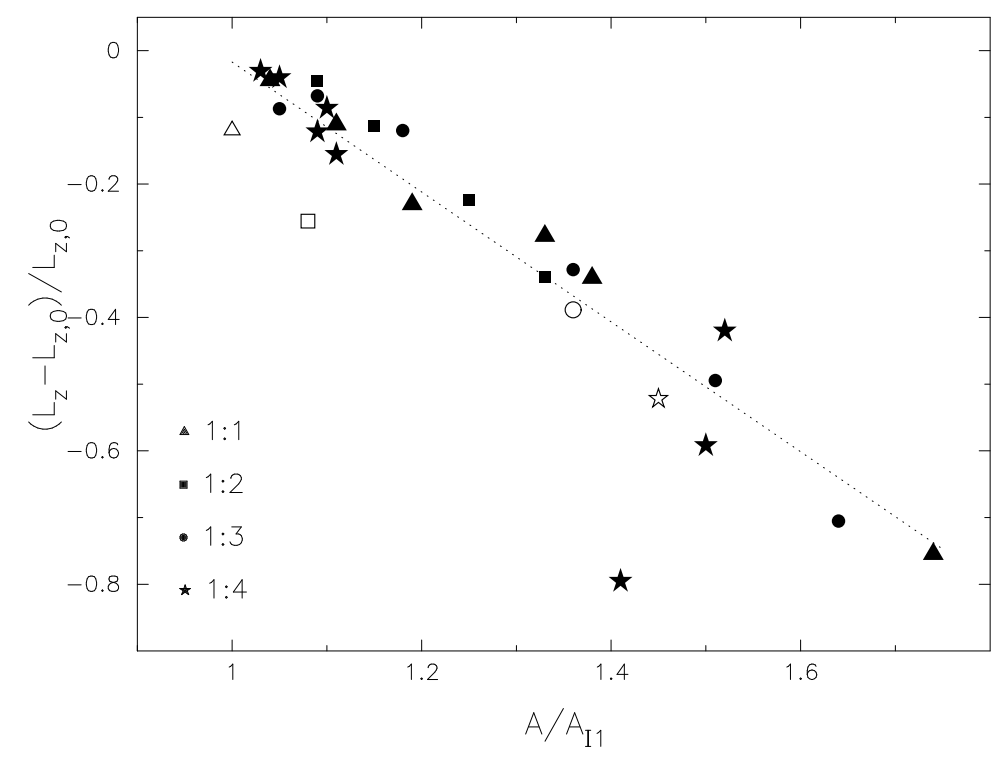

Figure 6.19: Total change of angular momentum $\Delta L_{z}$ of the stellar disc inside its initial cut-off radius as a function of bar strength normalized by the corresponding value of the isolated model. The different symbols represent interactions with different mass ratios. Parabolic and hyperbolic orbits are represented by open and filled symbols, respectively.

radius $r_{\text {cut }}$ and the induced bar strength, as shown in Fig. 6.19. The more angular momentum is removed from the disc, the stronger the regenerated bar becomes, in good agreement with what was found for isolated bars by Athanassoula (2003). We find the same correlation about equally strong when we plot the angular momentum change within the corotation radius. This important connection between the angular momentum exchange and the (re)generation process of the bar is described further in the discussion section.

\subsection{Discussion}

The regeneration of stellar bars triggered by galaxy interactions has been suggested as an additional scenario for the formation of bars and for explaining the observed frequency of bars along the Hubble sequence (Sellwood \& Moore 1999; Friedli 1999; Athanassoula 2000). So far only the formation of tidally induced bars in initially non-barred disc galaxies and the required conditions, under which such an event may occur, have widely been studied by means of numerical simulations (Byrd et al. 1986; Noguchi 1988; Salo 1991; Miwa \& Noguchi 1998; etc.). These results, however, need not necessarily apply to interactions with a formerly barred 


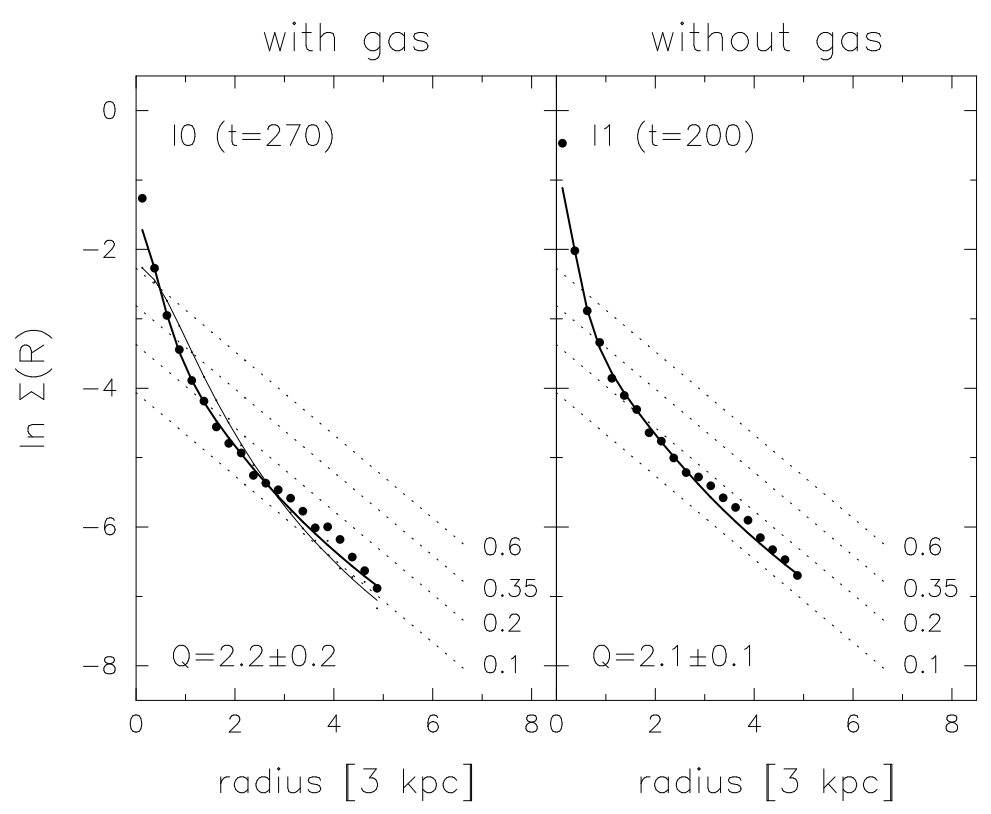

Figure 6.20: Logarithmic surface density of the stellar disc of models I0 (left-hand panel) and I1 (right-hand panel). The filled dots show the density distribution of the stellar discs and the thick full lines the best-fit of the corresponding Kuzmin-Toomre profile. The thin full line in the left-hand panel gives the density distribution of the initial stellar disc in model I0. We also show the different exponential discs used by Miwa \& Noguchi (1998) with dotted lines and give the mass of each disc in their model units. The value of the Toomre Q-parameter of the stellar disc is given at the bottom of each panel.

galaxy. Indeed, a former stellar bar likely might have changed the dynamics of the disc, i.e. increased the velocity dispersion in the stellar disc and changed both the density and angular momentum distribution. Therefore, the regeneration of stellar bars is subject to different conditions than the formation of a bar in an bar-unstable isolated disc or in a tidal interaction.

In this work we present numerical simulations starting with a host galaxy which has initially been bar unstable and in which the bar has been significantly weakened due to gas inflow before the interaction.

\subsubsection{Quantifying the interaction strength}

To quantify the strength of the interaction, we have introduced a parameter $\Theta$ for the interaction strength (Sec. 6.6.1), which basically takes the following quantities into account: 1) the mass of the companion, 2) the pericentric separation between the galaxies, and 3) the velocity of the companion at pericentre, defining a kind of inter- 


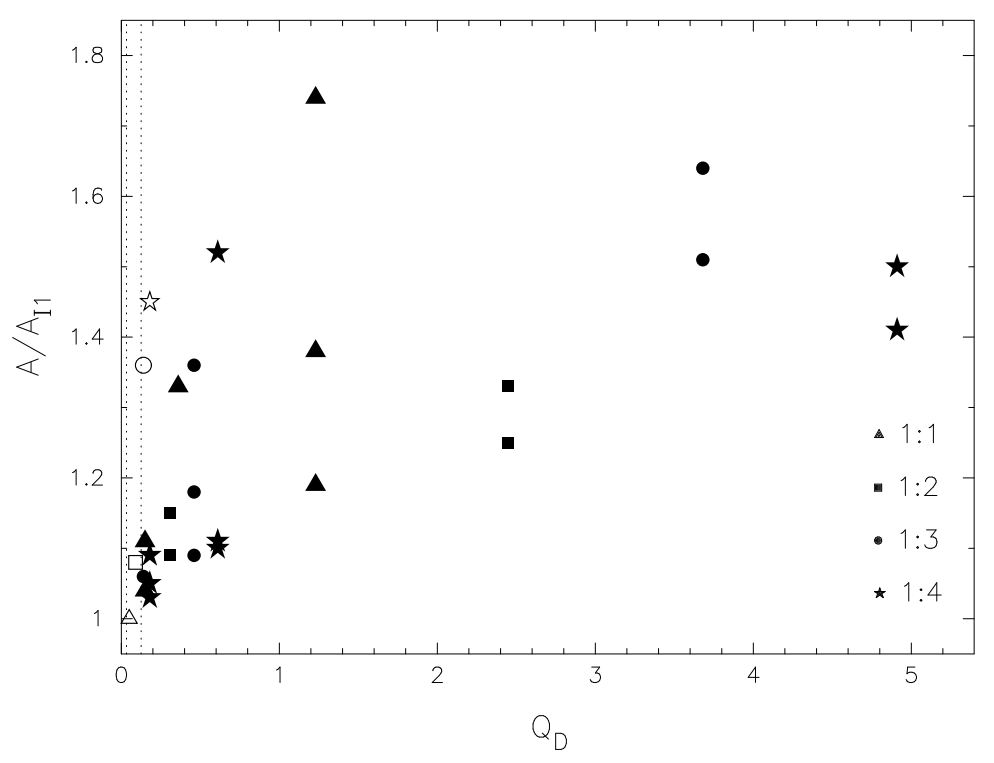

Figure 6.21: Normalized bar strength $\mathrm{A} / \mathrm{A}_{\mathrm{I} 1}$ versus the Dahari index $Q_{\mathrm{D}}$ (Dahari 1984) for interactions with different mass ratios. The layout is as in Fig. 6.15.

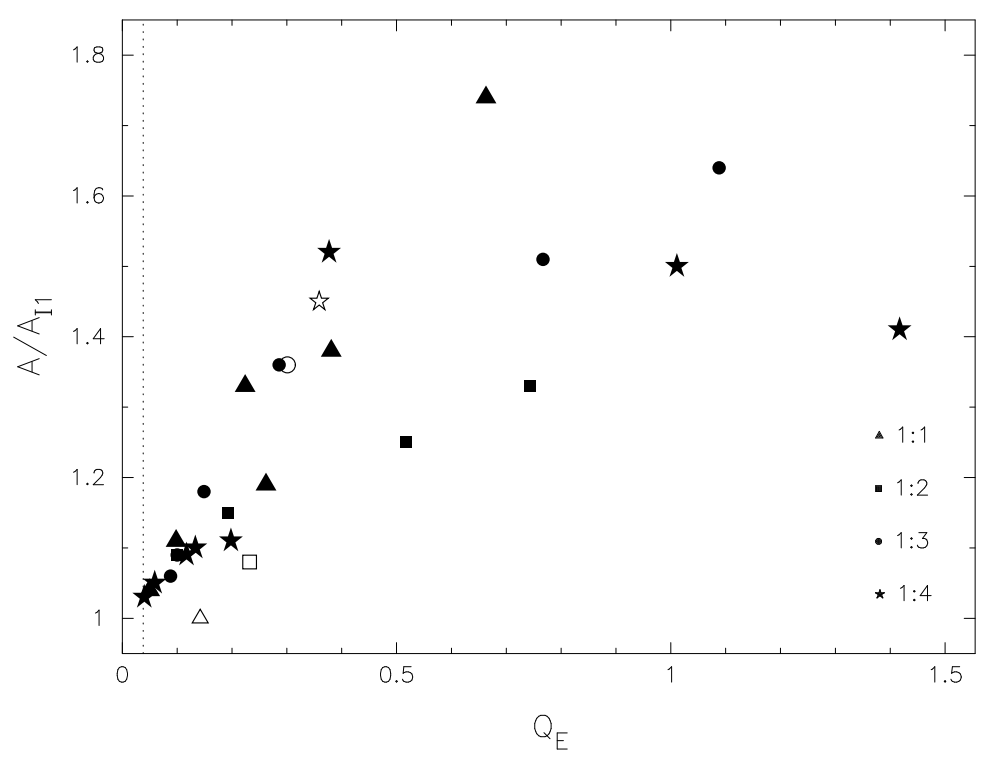

Figure 6.22: Normalized bar strength versus the interaction parameter $Q_{\mathrm{E}}$ (Elmegreen et al. 1991). The layout is as in Fig. 6.15.

action time-scale. With our definition (see Sec. 6.6.1), we find that the interaction strength $\Theta$ correlates well, i.e. roughly linearly, with the strength of the regenerated bars, which have been created in our purely stellar models. 
A different parameter for the interaction strength has been introduced by Dahari (1984) as an estimate of the direct tidal impulse:

$$
Q_{\mathrm{D}}=\left(M_{\text {comp }} / M_{\text {host }}\right) /\left(R_{\text {min }} / R_{\text {disc }}\right)^{3} \text {, }
$$

where $M_{\text {comp }}$ and $R_{\text {peri }}$ are the mass of the companion galaxy and the pericentric separation, respectively. $M_{\text {host }}$ is the mass of the host galaxy within the disc truncation radius $R_{\text {disc }}$. Salo (1991) has used the Dahari index as a quantitative measure for tidally induced bar formation in his $2 \mathrm{D}$ simulations and found a minimum value of $Q_{\mathrm{D}}$, depending on the specific host galaxy, above which a bar is formed by the interaction. The specific limiting value of $Q_{\mathrm{D}}$ Salo found depends strongly the central mass concentration of the host galaxy and cannot be easily transferred to our model. Furthermore, applying the Dahari index to our simulations we find neither a correlation between the strength of the interaction and the regeneration of the bar, nor a limiting value of $\mathrm{Q}_{\mathrm{D}}$ (see Fig. 6.21). The main drawback of the Dahari index is that it does not take into account the interaction orbit, i.e. the interaction time-scale. The Dahari index therefore does not seem to be sufficient to constrain the parameters necessary for the formation or regeneration of bars by galaxy interactions in general.

A more advanced parameter has been introduced by Elmegreen et al. (1991). This is based on the Dahari index, but includes also the ratio between an interaction time-scale $\Delta T$ and some dynamical time $T$ of the disc:

$$
Q_{\mathrm{E}}=Q_{\mathrm{D}} \times \frac{\Delta T}{T},
$$

where $T=R_{\text {gal }}^{3} / G M_{\text {gal }}$ and $\Delta T$ is the time it takes the companion to move by one radian relative to the hosts centre at pericentre time. These authors also report a limiting value $Q_{\mathrm{E}}=0.038$ for the formation of the bar by tidal interactions. Applying this parameter to our simulations we find a correlation between the interaction strength and the strength of the regenerated bar (see Fig. 6.22). This, however, is less pronounced than the corresponding one for our parameter $\Theta$, which takes more fully the interaction into account. The existence of a limiting parameter, as found by Salo (1991) and Elmegreen et al. (1991), however, might be attributed to the method for measuring the bar strength. For our purely stellar simulations we find a correlation between $\Theta$ and the induced bar strength rather than a limiting value of $\Theta$. Still, the exact correlation between $\Theta$ and the bar strength - as described in Sec. 6.6.1 - is likely to be model dependent.

\subsubsection{Radial redistribution of angular momentum}

Athanassoula (2003) argued that the redistribution of angular momentum is the driving agent for the evolution of an isolated barred galaxy. Both her analytical work and her numerical simulations show that galaxies that have exchanged more angular momentum should have a stronger bar, with a faster decreasing pattern speed. In 
our purely stellar models, we also find that, whenever bar regeneration occurs, it is always accompanied by a considerable loss of angular momentum from the disc (see Fig. 6.18). Indeed, as in the case of isolated galaxies (Athanassoula 2003), there is a tight correlation between the strength of the bar and the angular momentum lost by the inner disc. This argues that angular momentum exchange is tightly linked to the bar formation process, independent of whether that is spontaneous, or driven.

The actual exchange process, however, is not always the same. In isolated discs angular momentum is emitted by particles in resonance with the bar in the inner part of the disc (within corotation), and, to a lesser extent and if there is a considerable bar growth, by non-resonant particles in that region. This is absorbed by particles in the outer disc and halo. In this case there is only one pattern speed, that of the bar, since the spirals, which could in principle have a different pattern speed (Tagger et al. 1987; Sygnet et al. 1988), have died away in the early parts of the simulations. Thus resonances are well defined. This is certainly not the case here. Besides the bar pattern speed, there is the driving frequency of the interaction, which changes with time. Furthermore, the forcing from the companion is of comparable strength than that of the bar, and the companion itself participates actively in the angular momentum exchange. Thus, in contrast to the isolated cases, we do not find the bar corotation radius to separate disc angular momentum emitters from disc absorbers.

The bar regeneration process can be be understood in terms of basically the same angular momentum considerations as described by Athanassoula (2003) for isolated disc galaxies. The bar in an isolated disc can get stronger and lose angular momentum basically by four different effects, which should be linked to each other. First, particles which were on quasi-circular orbits outside the bar get trapped into elongated orbits in its outer part and thus the bar becomes longer. Secondly, the orbits trapped in the bar could get thinner and make the bar thinner, too. Third, more mass could be get trapped on periodic orbits in the bar. And finally, the bar can of course lose angular momentum by slowing down. These effects should also be present in our case as well, where the loss of disc angular momentum is predominantly driven by the tidal perturbation of the companion. We will discuss here how much the different effects contribute to the regeneration of the stellar bars in our simulations. Since the analytical calculations cannot make any statements about this, we will base the discussion on the dynamical properties and orbital structure of our specific models.

In Fig. 6.23 (right-hand column, upper panel) we show a direct comparison of the characteristic diagrams of the purely stellar models I1 and I1 C4 p at the end of each run, respectively. The characteristic diagram of the isolated and the perturbed case look very similar in terms of the layout of the main periodic orbits. The main difference, however, is that both the $x_{1}$ and the $x_{2}$ orbits extend to higher energies after the interaction. This is especially obvious for the part of the $x_{1}$ characteristic around $E_{\mathrm{J}}=-0.75$, where the value of the $y$ intercept increases strongly with $E_{\mathrm{J}}$. This clearly moved towards higher energies. In Fig. 6.24 (right-hand column) we 


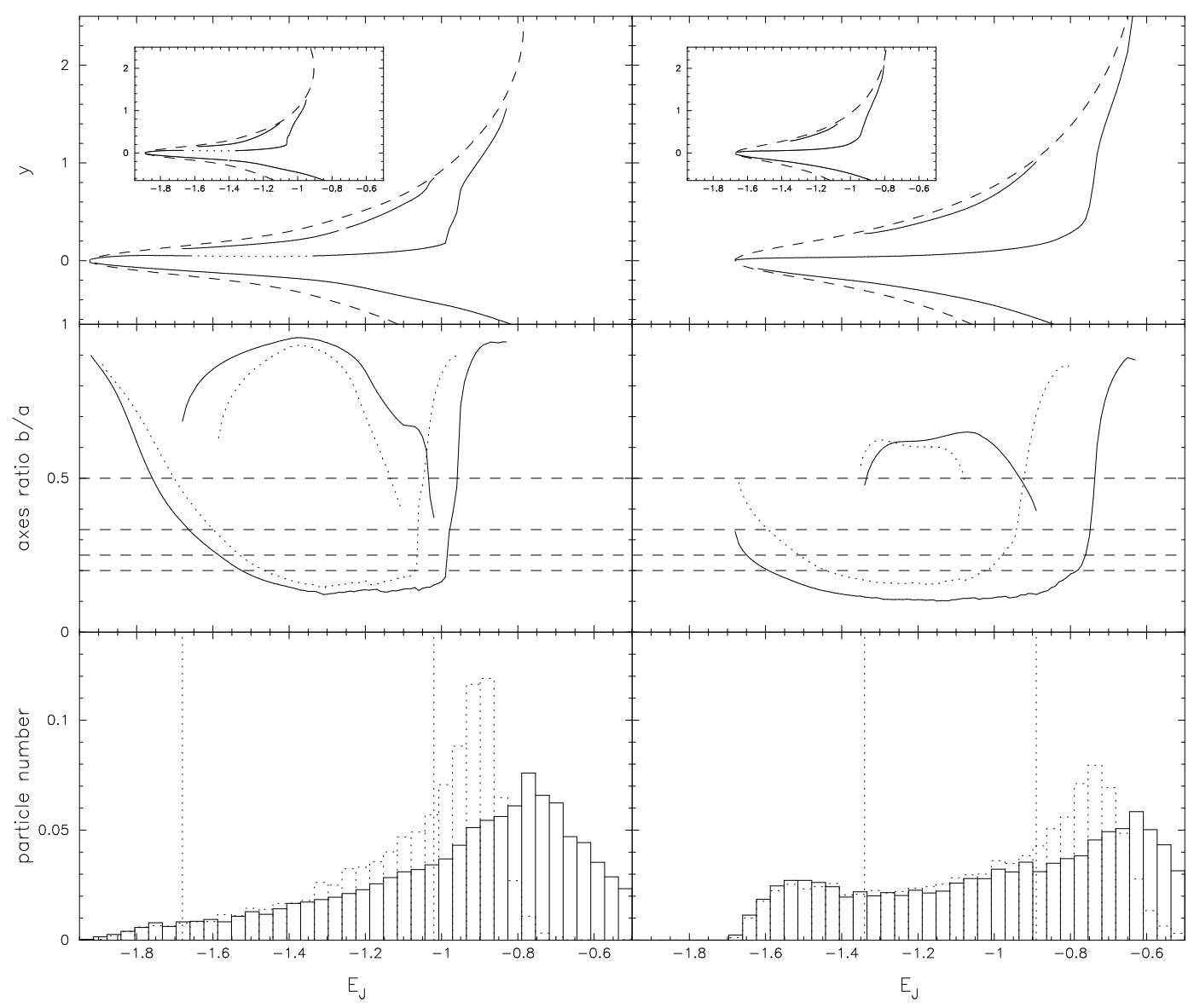

Figure 6.23: Comparison of models I0 C4 p (with gas; left-hand side) and I1 C4p (without gas; right-hand side). The upper panels show the characteristic diagram at the end of the run of the interaction model (main panel) and of the corresponding isolated model (subpanel). The layout of the diagrams is the same as for, e.g., Fig. 6.3. Instability regions in the characteristic curves are indicated by dotted lines. In the middle panels we show the axial ratios $b / a$ of the $x_{1}$ and $x_{2}$ orbit families, where $a$ and $b$ are the major and minor axis, respectively. The full and dotted lines show the results of the interaction and the isolated model, respectively. The horizontal dashed lines indicate axes ratios of $1: 2,1: 3,1: 4$ and $1: 5$. The bottom panels show a histogram of the number of stellar disc particles within corotation per energy interval, normalized by total number of stellar disc particles within corotation in the corresponding isolated model. The vertical dotted lines mark the region in which the $x_{2}$ orbits is present in the perturbed models.

show some examples of the periodic orbits with different Jacobian energies $E_{\mathrm{J}}$ of 

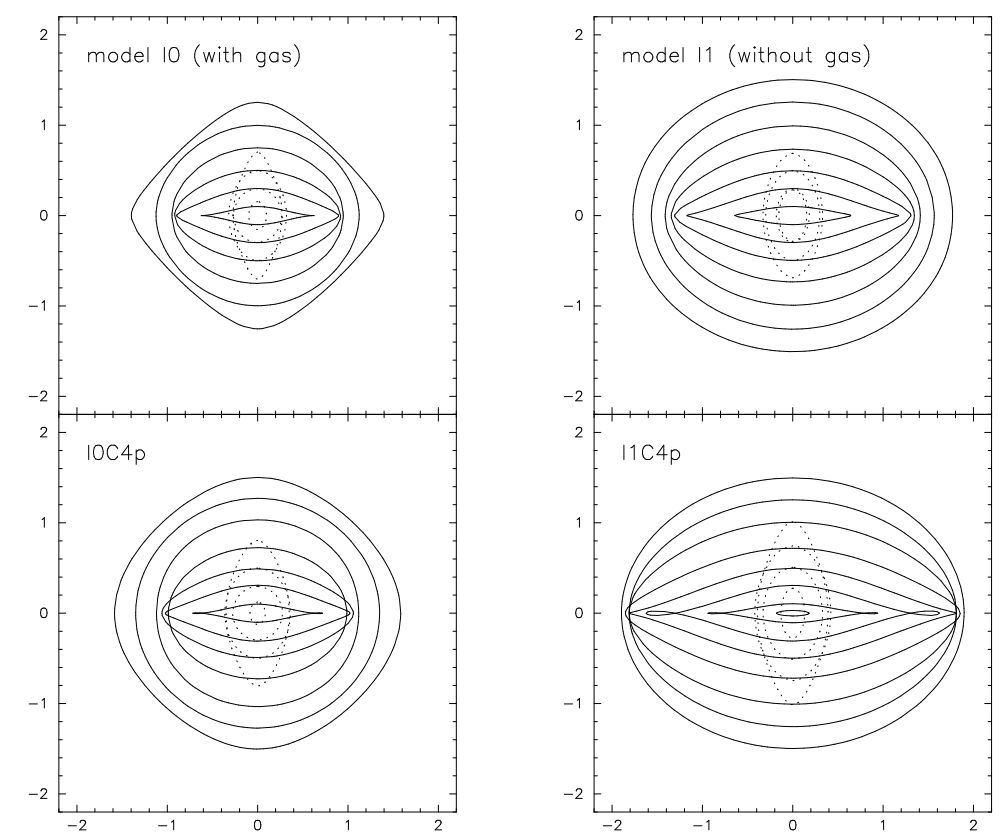

Figure 6.24: Concentric orbits of the $x_{1}$ (full lines) and $x_{2}$ (dotted lines) families. The left- and the right-hand column show the models with and without gas, respectively. In the upper panels we show the isolated models I0 and I1 and in the lower panels models I0 C4 p and I1 C4 p.

both the $x_{1}$ and the $x_{2}$ family. We find that the $x_{1}$ orbits become longer and, to some smaller extent though, also thinner after the interaction. This becomes clearer in Fig. 6.23 (middle panel), in which we plot the axial ratio $b / a$ of the orbits, where $a$ and $b$ denote the major and minor axis, respectively, as a function of $E_{\mathrm{J}}$. The main effect, however, is the lengthening of both the $x_{1}$ and the $x_{2}$ orbits towards higher energies and, taking into account the corresponding characteristic diagram, also in radial extent. For the $x_{1}$ orbits we also notice some lengthening towards lower energies, as well. Owing to these effects the bar gets both more centrally concentrated and more extended to large radii.

The thinning of the periodic orbits, which is less pronounced in our models than the lengthening, can be understood by the analytic calculations by LyndenBell (1979; hereafter LB79) in the context of gradual bar growth in isolated disc galaxies. As described in LB79, the mean circular frequency $\Omega_{\mathrm{c}}$ of most disc stars in the central and/or inner disc region is much higher than the pattern speed $\Omega_{\mathrm{p}}$ of the weak periodic perturbation, which is considered to be bar-like in this case. In the frame of reference corotating with the perturbation, the fast motion $\Omega_{\mathrm{c}}-\Omega_{\mathrm{p}}$ of the stars on near-circular orbits is not considerably affected by the weak perturbation and therefore the fast action variable $J_{\mathrm{f}}$ (see LB79) is approximately constant. 
As shown by Lynden-Bell, angular momentum is removed from the orbits, whose elongation leads the perturbing bar-like potential. Near-resonant orbits in the inner disc region, which reside in the abnormal region (see Fig. 2 in LB79), i.e. the region where $\Omega_{i}=\Omega_{\mathrm{c}}-\frac{1}{2} \kappa$ of the orbit decreases as its angular momentum decreases with constant $J_{\mathrm{f}}$, will align with the perturbation and become more eccentric, supporting the growth of the bar structure. As pointed out by Lynden-Bell, this way the bar shape also becomes more eccentric, but the length of the bar will not change significantly. The main difference to the scenario described in LB79 is that in our case, the perturbation is not periodic, since it is due to both the companion and the bar, and the angular frequency of the former is a function of time.

We finally check which orbits are important for making the bar. In the lower panel of Fig. 6.23 we therefore plot the number of particles as a function of $E_{\mathrm{J}}$, normalized by the total disc particle number within corotation of the isolated model I1. This way we get information about how the principal orbits at different energies are populated in our model. As can be seen from this plot, the total number of stellar particles within the corotation radius increased by less than 10 per cent. We conclude that the mass of the bar does not increase significantly. The main effect we find is that particles with energies close to the vertical $x_{1}$-branch move towards higher energies or higher radial extent, taking into account the characteristic diagram.

By tracking individual particle orbits in the corresponding potential and plotting their average y-intercept versus the Jacobian energy $E_{\mathrm{J}}$ in the characteristic diagram, we find that the $x_{2}$ orbits in the purely stellar models are only populated by a very small fraction of particles. This result is consistent with Fig. 6.25, in which we show the surfaces of section of model I1 C4 $\mathrm{p}$ at the end of the run for different $E_{\mathrm{J}}$. As can be clearly seen, the $x_{1}$ family dominates phase space over a wide range in energy and the $x_{2}$ orbits are almost not present.

\subsubsection{Influence of the gas}

Interactions which are strong enough to induce a bar in our purely stellar models are not sufficiently strong to induce a bar in the $N$-body/SPH models. In fact it has not been possible to induce a bar in any of our dissipative models, and we will here discuss the possible reasons for this. The stellar disc component in these simulations loses angular momentum, as was found also in the purely $N$-body models (see Fig. 6.8 and 6.18). The gas in the inner parts, however, gains angular momentum, so that the inner parts of the disc may gain a small amount of angular momentum (see Fig. 6.9), or if they lose, it is considerably less than the corresponding quantity for the purely stellar case. Furthermore, as was shown in Fig. 6.7 the interaction is accompanied by a significant inflow of gas towards the central disc. These two effects, coupled, prevent the regeneration of the bar. In this section we discuss how the increase of the central mass concentration affects the orbital structure of the disc and thus helps prevent the regeneration of the bar in these models. 

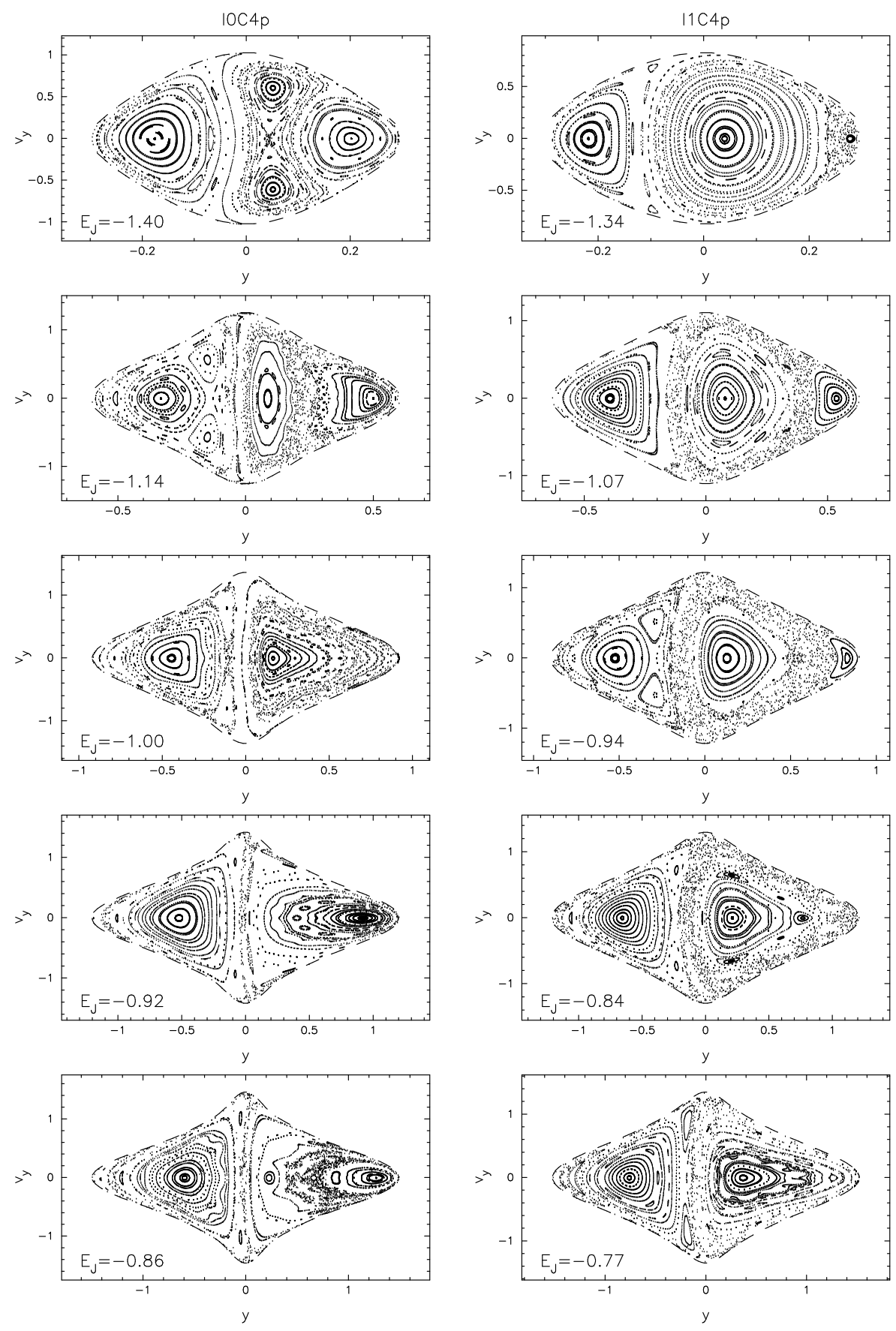

Figure 6.25: Surfaces of section for models I0 C4p (with gas; left-hand column) and I1 C4p (without gas; right-hand column) at the end of the runs. The values of the Jacobian energy $E_{\mathrm{J}}$, given in the bottom left-hand corner of each panel, have been chosen such as to give roughly the same range in $y$ for each row. In the purely stellar model the phase space is dominated by the $x_{1}$ and $x_{4}$ orbits at low Jacobian energies, while in the models with gas the phase space is mainly dominated by the $x_{2}$ and irregular orbits. 
In Fig. 6.23 (left-hand column) we compare models with gas, before and after the interaction. In particular, for models $\mathrm{I} 0$ and $\mathrm{I} 0 \mathrm{C} 4 \mathrm{p}$, we compare the characteristic diagrams (upper panel), the axial ratio of the $x_{1}$ and $x_{2}$ orbits (middle panel) and the particle number per energy range (bottom panel). In contrast to the models without gas (right column) the characteristic diagram of the models with gas extends more to lower energies, as would be expected from Fig. 6.7 and Sec. 6.4, that show clearly that the density distribution is centrally more concentrated in models with gas. Furthermore, the extent of the $x_{2}$ orbits is considerably larger, and the $x_{1}$ orbits have a sizeable instability strip between roughly $E_{\mathrm{J}}=-1.34$ and -1.66 .

Information on the axial ratio of the $x_{1}$ and $x_{2}$ orbits and on their extent is given in the middle panels of Figs 6.23 and 6.24. We see clearly that the differences between the cases before and after the interaction are much smaller than for the purely stellar case. The $x_{1}$ orbits are a bit thinner after the interaction and the $x_{2}$ orbits have slightly larger radial extent, but the differences are small. From Figs 6.23 and 6.24 we see that the $x_{1}$ orbits are less elongated than the corresponding orbits in the models without gas. This is particularly true at the highest and lowest energies. The extent of the elongated orbits is also considerably shorter. Also the $x_{2}$ orbits in the central region, i.e. at lower energies, are much rounder in the case with gas.

The number of stellar particles within corotation in models with gas (Fig. 6.23; bottom panel) have increased by roughly 12 per cent compared to the corresponding isolated model. Particles move from the intermediate energy region to the high energy one, and also, though to a lesser amount, to the low energy central region, similar to what is found in the models without gas.

Comparing the SOSs of models with and without gas (I0 C4 p and I1 C4 p, respectively) after the interaction (Fig. 6.25), we note that the area corresponding to $x_{2}$ orbits is much smaller in models with gas. On the other hand the area corresponding to chaotic motion and the area corresponding to $x_{1}$ orbits is considerably larger. The chaos in the cases with gas is due to the instabilities of the $x_{1}$ orbits, discussed above. The larger area covered by the $x_{2}$ orbits is in agreement with the fact that the $x_{2}$ characteristic is much more extended. To pursue this further we followed in the frozen potential the orbits of particles with initial conditions from the simulation, as we had already done for the simulations without gas. We find a notable difference. Namely there is now an indication that, contrary to the purely stellar case, a nonnegligible fraction of the orbits is trapped around $x_{2}$ orbits. This difference can be understood as a result of the induced gas-inflow (see Fig. 6.7), in accordance with the results found in numerical simulations of isolated gas-rich barred galaxies (see, for example, Friedli \& Benz 1993; Berentzen et al. 1998). Owing to the growing central mass concentration the $x_{2}$ orbits cover a larger phase-space volume at the expense of the $x_{1}$ orbits.

Thus the fact that bars cannot be regenerated in simulations with gas can be understood with the help of the many differences between the two models, described above. There is considerably less angular momentum loss from the inner disc material, if this is not a gain. The interaction brings considerably less change of the 
shape and extent of the $x_{1}$ orbits, but renders them unstable over a considerable energy interval, thus introducing a considerable amount of chaos. Finally, due to the increased central concentration, the importance of the $x_{2}$ population is considerably increased. The coupling of these three anti-bar effects prevents the regeneration of a bar component.

\subsubsection{Properties of regenerated bars}

As a next step we discuss the properties of the regenerated bars compared to the ones formed by the bar instability in isolated discs. Contrary to Miwa \& Noguchi (1998), we find no clear evidence for qualitative differences between the two types of bars, while we do find clear evidence for similarities. The orbital differences between the two types of bars, discussed in the two previous subsections are quantitative, rather than qualitative, since they pertain only to the extent of the families and the shape of the corresponding orbits. The role of the resonances seems the same.

Athanassoula (2003) found a correlation between the bar strength and the pattern speed of bars in isolated disc galaxies. In order to compare the properties of the bars formed in our models and the ones in isolated discs, we first calibrate our model units appropriately, as proposed in Athanassoula \& Misiriotis (2002), and then compared them to the ones in Athanassoula (2003). The result is shown in Fig. 6.26, which shows that the area covered by the driven bars is not separated by that covered by the isolated bars. This argues strongly for the similarity between the dynamical properties of the two types of bars, and the difficulty to distinguish between them.

Elmegreen \& Elmegreen (1985) found two different types of major axis surface density profiles, the exponential and the flat ones, and Noguchi (1996) argued that this distinction could be due to the fact that the first type is found in spontaneous bars and the second in driven ones. We believe that the difference is due to the bar strength, rather than its origin, since Athanassoula \& Misiriotis (2002) showed that both types of profiles could be found in isolated galaxies: the flat profiles occurring in the stronger bars and the exponential ones in less strong ones.

Apart from these differences, we find agreement with the results of Miwa \& Noguchi (1998). Thus, in our purely stellar simulations we find a roughly linear correlation between the pattern speed of the regenerated bar and its strength, or, accordingly, the interaction parameter $\Theta$ (see Figs 6.17 and 6.15, respectively). In other words, stronger bars - regenerated by the interaction - have lower pattern speeds. This result is in agreement with the simulations of Miwa \& Noguchi (1998), who find a similar correlation - less strict though - between the pattern speed of the induced bars and the companions mass. In both cases, i.e. the formation (Miwa \& Noguchi 1998) and regeneration of stellar bars (see Fig. 6.17) by tidal interactions, the bars always have lower pattern speeds than the bars formed spontaneously in the corresponding isolated models.

In agreement with Noguchi (1987) and Gerin et al. (1990) we find that tidal 


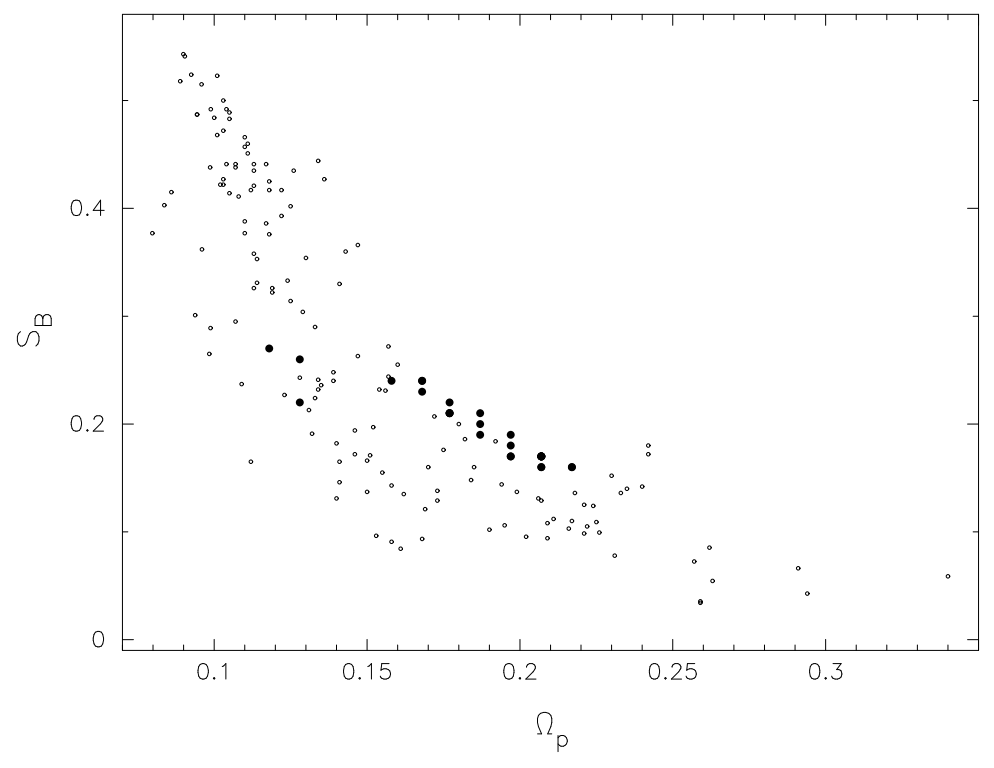

Figure 6.26: Bar strength $S_{\mathrm{B}}$ versus pattern speed $\Omega_{\mathrm{p}}$ in units used by Athanassoula $\&$ Misiriotis (2002). The dots and bullets show the results of the isolated models of Athanassoula (2003) and our purely stellar interaction models, respectively.

bars are not transient, but long-lived, as are spontaneous bars. Thus bars which are observed at high redshifts (Sheth et al. 2003) may have formed by galaxy interactions and thus played an important role in the evolution of disc galaxies (Sheth et al. 2003).

\subsubsection{Numerical considerations}

The simulations presented in this work have been performed with a rather low particle number of the host galaxy, because of the relatively large sample of simulations. On the one hand the induced numerical noise in general supports the bar formation process, while on the other it will heat the disc, and thus make the bar regeneration more difficult. We trust, however, that a higher number of particles would make only quantitative, and not qualitative differences. Thus the physical results should remain unchanged.

The companion galaxy in our simulations has always been approximated by a softened point mass. For simulations in which the companion passes outside the halo of the host galaxy, this approximation is well suited. For simulations in which the companion crosses the halo, however, dynamical friction can be sufficiently strong to change the orbit of the companion and finally lead to a merger between the two galaxies. 


\subsection{Summary}

In this paper we used numerical simulations to investigate the regeneration of a stellar bar by tidal encounters. The host galaxy has been chosen to be initially bar unstable and forms a large-scale bar during its early evolution. Before the interaction with the companion galaxy the bar significantly weakened, owing to the bar-driven gas-inflow towards the central disc region. For the simulations presented in this work we used two different types of host galaxies, i.e. one gas-rich disc model and one without gas. The encounters in our simulations have been chosen to be prograde in respect to the rotation in the host disc and co-planar with the discs equatorial plane. The mass and the orbit of the companion have been varied in order to cover a large parameter space.

We found that interactions, which are sufficiently strong to regenerate the bar in the purely stellar models, do not lead to a regeneration in the dissipative models. The regenerated bars in our simulations are long-living phenomena and by no means only transient, i.e. that (regenerated) bars formed by interactions may indeed contribute to the number of barred spirals at high redshifts and in the local Universe.

We have shown that the strength of the regenerated bars increases with the interaction strength. Owing to the tidal perturbation, angular momentum is removed from the disc. In fact, the whole disc within its initial cut-off radius loses angular momentum, in contrast to what is found for an isolated disc, where the corotation radius of the bar separates disc regions loosing and gaining angular momentum. The amount of angular momentum removed from the disc shows a clear correlation with the interaction strength. We argued that the main effect of this angular momentum loss is a significant extension of the region where bar-supporting orbits exist, resulting in a lengthening of the bar. As a further, though somewhat less important, effect, we found also a thinning of both the bar-supporting orbits and the bar itself.

The regenerated bars are generally slow-rotators and have lower pattern speeds than the bars in the corresponding isolated models. Furthermore, we found strong correlation between the strength and the pattern speed of the bar. This correlation is in very good agreement with the correlation found for bars in isolated discs. This is one of the pieces of evidence that the regenerated bars are qualitatively similar to those formed in isolated discs and thus cannot easily (if at all) be distinguished from them by their dynamical properties.

In contrast to the purely stellar simulations presented in this work, it has not been possible to regenerate the bar in our models including gas. We argued that owing to the interaction additional gas is driven towards the centre of the disc, which finally prevents the regeneration process. We concluded that the regeneration of stellar bars by galaxy interactions seems to be a reasonable mechanism, provided the external forcing is sufficiently strong. 


\subsection{Acknowledgments}

We would like to thank Albert Bosma for interesting discussions, and J.C. Lambert and $\mathrm{C}$. Theis for their computer assistance. IB acknowledges support from DFG grant Fr 325/48-2, /48-3 and Volkswagen Foundation grant I/76 520. He thanks the DFG for funding the grape facilities at the Sternwarte Göttingen. $\mathrm{CH}$ acknowledges support from grants HST-AR-09546.02-A, NSF-AST-0206251, GSU-FRG-2002, and DFG Fr 325/39-1, /39-2. EA would like to thank the IGRAP, the INSU/CNRS and the University of Aix-Marseille I for funds to develop the grape facilities used for part of the calculations in this paper. The final draft of this paper was written while E.A. was in I.N.A.O.E. She thanks the I.N.A.O.E. staff for their kind hospitality and ECOS-Nord/ANUIES for a travel grant that made this trip possible.

\subsection{Bibliography}

Athanassoula E., 2000, in Combes F., Mamon G.A., Charmandaris V., eds, ASP Conf. Ser. Vol. 197, Dynamics of Galaxies: from the Early Universe to the Present. Astron. Soc. Pac., San Francisco, p. 455

Athanassoula E., 2002, ApJ, 569, L86

Athanassoula E., 2003, MNRAS, 341, 1179

Athanassoula E., Misiriotis A., 2002, MNRAS, 330, 35

Athanassoula E., Sellwood J.A., 1986, MNRAS, 221, 195

Barnes J.E., Hernquist L.E., 1991, ApJ, 370, 65

Berentzen I., Athanassoula E., Heller C.H., Fricke K.J., 2003, MNRAS, 341, 343

Berentzen I., Heller C.H., Shlosman I., Fricke K.J., 1998, MNRAS, 300, 49

Binney J., Tremaine S., 1987, Galactic Dynamics. Princeton Univ. Press

Byrd G.G., Valtonen M.J., Valtaoja L., Sundelius B., 1986, A\&A, 166, 75

Contopoulos G., Grosbø1 P., 1989, A\&ARv, 1, 261

Contopoulos G., Papayannopoulos Th., 1980, A\&A, 92, 33

Dahari O., 1984, AJ, 89, 966

de Vaucouleurs G., 1963, ApJS, 8, 31

Elmegreen B.G., Elmegreen D.M., 1985, ApJ, 288, 438

Elmegreen D. M., Sundin M., Sundelius B., Elmegreen B., 1991, A\&A, 244, 52

Eskridge P.B., Frogel J.A., Pogge R.W., et al., 2000, AJ, 119, 536

Friedli D., 1999, in Beckman J.E., Mahoney T.J., eds, ASP Conf. Ser. Vol. 187, The Evolution of Galaxies on Cosmological Timescales. Astron. Soc. Pac., San Francisco, p. 88

Friedli D., Benz W., 1993, A\&A, 268, 65

Gerin M., Combes F., Athanassoula E., 1990, A\&A, 230, 37

Heller C.H., 1991, PhD thesis, Yale Univ.

Heller C.H., Shlosman I., 1994, ApJ, 424, 84

Hohl F., 1971, ApJ, 168, 343 
Kuzmin G., 1956, Astron. Zh, 33, 27

Lynden-Bell D., 1979, MNRAS, 187, 101L

Lynden-Bell D., Kalnajs A.J., 1972, MNRAS, 157, 1L

Miller R.H., Prendergast K.H., Quirk W.J., 1970, ApJ, 161, 903

Miwa T., Noguchi M., 1998, ApJ, 499, 149

Monaghan J.J., 1992, ARA\&A, 30, 543

Noguchi M., 1987, MNRAS, 228, 635

Noguchi M., 1988, A\&A, 203, 259

Noguchi M., 1996, ApJ, 469, 605

Ostriker J.P., Peebles P.J.E., 1973, ApJ, 186, 467

Plummer H.C., 1911, MNRAS, 71, 460

Salo H., 1991, A\&A, 243, 118

Sellwood J. A., 1981, A\&A, 99, 362

Sellwood J. A., Moore E.M., 1999, ApJ, 510, 125

Sheth K., Regan M.W., Scoville N., Sparke L., 2003, ApJL, in press

Shlosman I., Begelman M.C., Frank J., 1989, Nat, 338, 45

Spitzer L., 1942, ApJ, 95, 329

Steinmetz M., 1996, MNRAS, 278, 1005

Sugimoto D., Chikada Y., Makino J., Ito T., Ebisuzaki T., Umemura M., 1990, Nat, 345,33

Sygnet J.F., Tagger M., Athanassoula E., Pellat R., 1988, MNRAS, 232, 733

Tagger M., Sygnet J.F., Athanassoula E., Pellat R., 1987, ApJ, 318, 43L

Toomre A., 1963, ApJ, 138, 385 
7. The accuracy of the pattern speed in numerical simulations of barred galaxies

\author{
I. Berentzen $^{1,2}$, E. Athanassoula ${ }^{2}$, C.H. Heller ${ }^{3}$, and K.J. Fricke ${ }^{1}$ \\ ${ }^{1}$ Universitäts-Sternwarte, Geismarlandstraße 11, D-37083 Göttingen, Germany \\ ${ }^{2}$ Observatoire Astronomique de Marseille, 2 Place Le Verrier, F-13248 Marseille Cedex 4, \\ France \\ ${ }^{3}$ Georgia Southern University, Department of Physics, Statesboro, GA 30460, U.S.A.
}

\begin{abstract}
We use fully self-consistent $N$-body/smoothed-particle-hydrodynamics simulations to study the influence of different numerical effects on the evolution of bars in models of isolated galaxies. We argue that the evolution of the bar's pattern speed is affected by both the intrinsic numerical noise owing to the $N$-body realisation and the numerical accuracy of the code, e.g. in the force calculation and in the time-stepping scheme. These effects can lead to a difference in the evolution of the pattern speed up to 13 per cent. We conclude that the pattern speeds determined from models today which have only a few tens-of-thousands of particles and/or moderate accuracy in the force computation are not very robust and care should be taken not to over-interpret their values and evolutionary behaviour.
\end{abstract}

\title{
7.1 Introduction
}

The angular velocity or pattern speed (hereafter $\Omega_{\mathrm{p}}$ ) at which bars rotate in disc galaxies is one of the fundamental parameters determining the structure of barred galaxies. The rotating bar introduces dynamical resonances in the disc, and it has been shown that the shape of the bar and its pattern speed are directly linked to the exchange of angular momentum in the disc and between the disc and spheroidal components like bulge or halo (Athanassoula 2003).

The direct measurement of $\Omega_{\mathrm{p}}$ from observations, however, is very difficult. Several methods have been suggested and applied to determine the pattern speed of bars. The Tremaine-Weinberg method (Tremaine \& Weinberg 1984), which is 
based on the continuity equation of a tracer population, allows one to derive $\Omega_{\mathrm{p}}$ from long-slit spectra and photometric light profiles along the bar's major axis. The Tremaine-Weinberg method is, however, strongly sensitive to errors in the position angle of the disc (Debattista 2003). Another way is to use the non-circular motion produced by the bar and spiral structure to locate the radius of corotation in the disc (Canzian 1993) and thus the pattern speed. Additionally one can identify characteristic morphological structures, like inner and outer rings in the disc, with the location of resonances induced by the bar (see, for example, Elmegreen, Elmegreen \& Seiden 1989; Elmegreen \& Elmegreen1990; Knapen et al. 1992; etc.). If the potential of the galaxy can be derived, e.g. from near-infrared data, one can use numerical hydrodynamical simulations (see, for example, Sempere et al. 1995a, 1995b; Lindblad, Lindblad \& Athanassoula 1996; ; Lindblad \& Kristen 1996; Laine, Heller \& Shlosman 1998; etc.) to find $\Omega_{\mathrm{p}}$. The bar's pattern speed can be varied until the observed gas-flow patterns are reproduced sufficiently well. The morphology and the inflow are dependent on the hydrodynamical and numerical parameters used in the models (Patsis \& Athanassoula 2000).

To study the evolution of bar pattern speeds one needs to turn to self-consistent $N$-body simulations. In such simulations the pattern speed can easily be derived from the change of the bar's phase angle in the disc. For instance, such simulations have been used to study the slow-down of bars (see, for example, Hernquist \& Weinberg 1992; Athanassoula 1996; Debattista \& Sellwood 1998, 2000; Athanassoula 2003).

In this paper we examine the simulations ability to produce robust and reproducible pattern speeds. The models we run are that of of barred galaxies using fully self-consistent $N$-body/smoothed-particle-hydrodynamics (hereafter SPH) simulations. In Sec. 7.2 we describe the numerical methods and initial conditions used for the models presented in this work. In Sec. 7.3 we then provide a direct comparison of the results of our reference model from GRAPE-3 and GRAPE-5 in comparison with the treecode, using different resolutions. Furthermore we present different tests to rule out different sources of error in the code. In Sec. 7.4 we study the effect of the initial conditions used for the $N$-body realisation of the models. Finally, in we describe in Sec. 7.5 further numerical effects introduced by the code. We discuss the results in Sec. 7.7 and finally give a summary in Sec. 7.8.

\subsection{Numerical methods and initial conditions}

\subsubsection{Numerical methods}

To evolve both the collisionless component (representing the stars and the dark matter) and the dissipative component (representing the gas) we use an $N$-body combined with an SPH algorithm (see, for example, the review by Monaghan 1992). In particular we use the version of the hybrid $N$-body/SPH code of Heller (1991; 
see also Heller \& Shlosman 1994). This 3D algorithm employs such features as a spatially varying smoothing length, a hierarchy of time-bins to approximate individual time-steps and a viscosity 'switch' to reduce the effects of viscous shear. To compute the gravitational forces and neighbour interaction lists we use either the special-purpose hardware GRAPE-3AF and GRAPE-5 (Sugimoto et al. 1990; Steinmetz 1996) or a treecode.

\subsubsection{The GRAPE hardware}

The most time-consuming part in $N$-body simulations is the gravitational force calculation. In the direct method, i.e. the direct summation of the forces between the $N$ particles, the computational cost scales as $N(N-1)$. Hence several techniques have been developed, both from the numerical and the technical side, to obviate this bottleneck. There are the software-based methods, such as the Barnes \& Hut Treecode (Barnes \& Hut 1986) or the Very-Fast-Treecode (Dehnen 2000), which scale as $N \log N$ or $N$, respectively. Other software methods, like the grid-based or field-expansion methods, often have the disadvantage, that they set constraints on the geometry of the system. In contrast to these methods there is the hardware approach. For this special-purpose machines are constructed, such as the GRAPE (short for: GRAvity PipE) hardware series, in which the direct force calculation is hardwired on chips, thus greatly accelerating the force calculation. There exist different versions/generations of GRAPE-boards, of which the GRAPE-3 (Okumura et al. 1993) and GRAPE-5 (Kawai et al. 2000) are well suited for galaxy simulations. The performance and accuracy of the GRAPE-3 hardware is described in Athanassoula et al. (1998). The GRAPE-5 is the successor of the GRAPE-3 and provides a higher numerical accuracy in the force calculation and is about 10 times faster (Kawai et al. 2000). The GRAPE hardware furthermore supports hydrodynamical schemes like SPH and is thus widely being used in galactic and cosmological studies.

\subsubsection{Initial conditions}

Our galaxy model is initially composed of a stellar and gaseous disc, embedded in a spherical halo. The radial surface density of the disc follows a truncated KuzminToomre (hereafter KT) profile (Kuzmin 1956; Toomre 1963) with a radial scalelength $a_{\mathrm{d}}$. The disc vertical density profile follows the sech ${ }^{2}$ distribution of an isothermal sheet (Spitzer 1942). The halo is set up initially as a Plummer (hereafter Pl) sphere (Plummer 1911) with a radial scalelength $b_{\mathrm{h}}$. The initial model parameters are summarised in Tab. 7.1. The columns from left to right give the component, the type of density profile, the particle number, the total mass, the radial scalelengths $a_{\mathrm{d}}$ and $b_{\mathrm{h}}$, the cut-off radius $r_{\text {cut }}$, and the vertical scaleheight $z_{0}$ of each component.

The halo is then allowed to relax in the gradually introduced potential of the frozen disc. After the relaxation of the halo we assign velocities to the disc particles 
Table 7.1: Initial model parameters.

\begin{tabular}{lcccccc}
\hline Component & Type & $N_{\mathrm{d}}$ & $\mathrm{M}_{\mathrm{d}}$ & $a_{\mathrm{d}}$ & $\mathrm{r}_{\text {cut }}$ & $\mathrm{z}_{0}$ \\
\hline Disc & & & & & & \\
$\quad$ stars & $\mathrm{KT}$ & 13500 & 0.54 & 1.0 & 5.0 & 0.20 \\
gas & $\mathrm{KT}$ & 10000 & 0.16 & 1.0 & 5.0 & 0.05 \\
& & & & & & \\
\hline Component & Type & $N_{\mathrm{h}}$ & $\mathrm{M}_{\mathrm{h}}$ & $b_{\mathrm{h}}$ & $\mathrm{r}_{\text {cut }}$ & \\
\hline $\begin{array}{l}\text { Halo } \\
\quad \text { stars }\end{array}$ & $\mathrm{Pl}$ & 32500 & 1.30 & 5.0 & 10.0 & $\cdots$ \\
\hline
\end{tabular}

such that the disc is globally unstable to non-axisymmetric perturbations and will form a large-scale bar during its evolution. For a detailed description of the initial model see also Berentzen et al. (2003). In the following we will refer to this model as our reference model (hereafter model I0). In addition we have created three more models (hereafter I1, I2 and I3, respectively) based on this reference model. The new realisations have been constructed by changing the initial $(x, y)$ component of both the position and velocities of all particles, keeping the particles initial radius fixed and rotating it by some random angle about the $z$-axis. The angle of rotation for each has been determined using a random number generator with three different initial seeds.

In Tab. 7.2 we summarize some of the parameters of the different simulations presented in the paper. The columns from left to right give the method/hardware used, the opening angle $\theta$ of the treecode simulations, along with the models and special parameters used. The minimum and maximum time-steps in the hierarchical stepping scheme are $\tau_{\min }=0.02$ and $\tau_{\max }=1.0$, respectively, if not stated otherwise.

\subsubsection{Units}

The adopted units for mass, distance, and time are $M=6 \times 10^{10} \mathrm{M}_{\odot}, \mathrm{R}=3 \mathrm{kpc}$ and $\tau=10^{7} \mathrm{yr}$, respectively, for which the gravitational constant $\mathrm{G}$ is unity. The dynamical time is $\tau_{\text {dyn }} \equiv\left(r_{1 / 2}^{3} / \mathrm{G} M_{1 / 2}\right)^{1 / 2}=4.8 \times 10^{7}$ yr, where $M_{1 / 2}$ is half the hosts total mass and $r_{1 / 2}$ is the half-mass radius, which is, after relaxation of the halo, approximately $8.5 \mathrm{kpc}$ or in terms of the disc scalelength, $2.8 a_{\mathrm{d}}$. The initial stellar disc rotation period in these units then corresponds to $t_{\text {rot }} \equiv 2 \pi \tau_{\text {dyn }} \approx 3 \times 10^{8}$ yr. A fixed gravitational softening length of $\epsilon=0.375 \mathrm{kpc}$ is used for all particles ${ }^{1}$. An isothermal equation of state is used for the gas with a sound speed of $v_{\mathrm{s}}=12 \mathrm{~km}$ $\mathrm{s}^{-1}$. The corresponding thermal gas temperature is $10^{4} \mathrm{~K}$.

\footnotetext{
${ }^{1}$ Because the GRAPE hardware supports only a fixed gravitational softening for all particles, we apply a fixed $\epsilon$ for the treecode simulations, too.
} 
Table 7.2: Simulation description.

\begin{tabular}{lllc}
\hline Method & $\theta$ & Model(s) & comments \\
\hline GRAPE-3 & $\cdots$ & $\mathrm{I} 0, \mathrm{I} 1, \mathrm{I} 2, \mathrm{I} 3$ & $\cdots$ \\
GRAPE-5 & $\cdots$ & $\mathrm{I} 0$ & $\ldots$ \\
\hline GRAPE-3 & $\cdots$ & $\mathrm{I} 0$ & $t_{\mathrm{rst}}=60,180,220$ \\
GRAPE-3 & $\cdots$ & $\mathrm{I} 0$ & $\mathrm{SPH}$ with $1.5 \mathrm{~h}$ \\
\hline treecode & 0.1 & $\mathrm{I} 0, \mathrm{I} 1, \mathrm{I} 2, \mathrm{I} 3$ & $\cdots$ \\
treecode & 0.2 & $\mathrm{I} 0, \mathrm{I} 1, \mathrm{I} 2, \mathrm{I} 3$ & $\cdots$ \\
treecode & 0.3 & $\mathrm{I} 0, \mathrm{I} 1, \mathrm{I} 2, \mathrm{I} 3$ & $\cdots$ \\
treecode & 0.4 & $\mathrm{I} 0, \mathrm{I} 1, \mathrm{I} 2, \mathrm{I} 3$ & $\cdots$ \\
treecode & 0.5 & $\mathrm{I} 0, \mathrm{I} 1, \mathrm{I} 2, \mathrm{I} 3$ & $\cdots$ \\
treecode & 0.6 & $\mathrm{I} 0, \mathrm{I} 1, \mathrm{I} 2, \mathrm{I} 3$ & $\cdots$ \\
treecode & 0.7 & $\mathrm{I} 0, \mathrm{I} 1, \mathrm{I} 2, \mathrm{I} 3$ & $\cdots$ \\
treecode & 0.8 & $\mathrm{I} 0, \mathrm{I} 1, \mathrm{I} 2, \mathrm{I} 3$ & $\cdots$ \\
treecode & 0.9 & $\mathrm{I} 0, \mathrm{I} 1, \mathrm{I} 2, \mathrm{I} 3$ & $\cdots$ \\
treecode & 1.0 & $\mathrm{I} 0, \mathrm{I} 1, \mathrm{I} 2, \mathrm{I} 3$ & $\cdots$ \\
\hline treecode & 0.5 & $\mathrm{I} 0$ & $\tau_{\min }=0.01, \tau \max =1.0$ \\
treecode & 0.5 & $\mathrm{I} 0$ & $\tau_{\min }=0.01, \tau_{\max }=0.05$ \\
treecode & 0.5 & $\mathrm{I} 0$ & $\tau_{\min }=0.04, \tau_{\max }=1.0$ \\
treecode & 0.5 & $\mathrm{I} 0$ & $\tau_{\min }=0.06, \tau_{\max }=1.0$ \\
treecode & 0.7 & $\mathrm{I} 0$ & quadrupole on \\
\hline treecode & 0.15 & $\mathrm{I} 0$ & $\mathrm{G} 5$ resolution \\
treecode & 0.66 & $\mathrm{I} 0$ & $\mathrm{G} 3$ resolution \\
\hline hybrid & $\cdots$ & $\mathrm{I} 0$ & neighb. from tree \\
hybrid & 0.2 & $\mathrm{I} 0$ & gravity from tree \\
\hline
\end{tabular}

\subsubsection{Measuring the bar properties}

In the following we define the bar strength by the normalized $m=2$ Fourier component of the stellar mass distribution. For a given $m$, the Fourier coefficients are given by:

$$
A_{m}=\sum_{i=1}^{N_{\mathrm{d}}} m_{i} \cos \left(m \theta_{i}\right), \quad m=0,1,2, \ldots,
$$

and

$$
B_{m}=\sum_{i=1}^{N_{\mathrm{d}}} m_{i} \sin \left(m \theta_{i}\right), \quad m=1,2, \ldots,
$$


where the sum is taken over all stellar disc particles $N_{\mathrm{d}}$ and $\theta_{i}$ and $m_{i}$ are the polar position angle and the mass of the $i$-th particle, respectively. We then define the strength of the bar as:

$$
S_{\mathrm{B}}=\frac{\sqrt{A_{2}^{2}+B_{2}^{2}}}{A_{0}}
$$

and the phase-angle of the bar is derived as:

$$
\theta_{\mathrm{B}}=\frac{1}{2} \arctan \left(\frac{B_{m}}{A_{m}}\right)
$$

We measure the bar's amplitude $S_{\mathrm{B}}$ within a cylindrical radius $R=1.25$ and about one scaleheight of the stellar disc. Special care was taken not to include any power contributed by the stellar spiral arms. The pattern speed of the bar is derived directly from the phase angle as $\Omega_{\mathrm{p}}=\Delta \theta_{\mathrm{B}} / \Delta t$. We tested different radii in order to make sure that the measurement was not overly sensitive to the choice.

\subsection{Direct comparison of GRAPE-3 and -5 simula- tions}

In order to provide a direct comparison between simulations using the GRAPE-3 and GRAPE-5 hardware (hereafter G3 and G5, respectively), we first run our reference model $\mathrm{I} 0$ of an isolated barred galaxy on both machines. The initial conditions of the disc in our reference model have been chosen such as to be bar-unstable and form a strong bar after a few disc rotations. Owing to the torques of the growing bar, gas is driven towards the central region and accumulates in a central gaseous disc. The growing central mass concentration leads to a significant weakening of the bar during the further evolution. A detailed description of the reference model (G3 simulation) and its evolution can be found in Berentzen et al. (2003). From simple inspection by eye of the particle distribution and the isodensity contour plots, we find that the morphological evolution of the disc (not shown here) is basically identical in both simulations (G3 and G5).

Fig. 7.1 (upper panel) shows the evolution of the bar strength, as defined in the previous section, for the simulations G3 and G5. The bar strength during the evolution in both models is basically identical within the error bars. After the nonlinear growth-phase, the bar reaches its maximum strength at roughly $t=60$ and weakens significantly owing to the induced gas inflow. After the disc has settled down to a quasi-stable state again, the bar strength remains roughly constant till the end of the run, i.e. $t=300$, or $3 \times 10^{9} \mathrm{yr}$. The pattern speed $\Omega_{\mathrm{p}}$ of the bar (see Fig. 7.1, lower panel), however, shows clear differences between the two simulations. Essentially we can distinguish four different phases in the evolution of $\Omega_{\mathrm{p}}$ : 


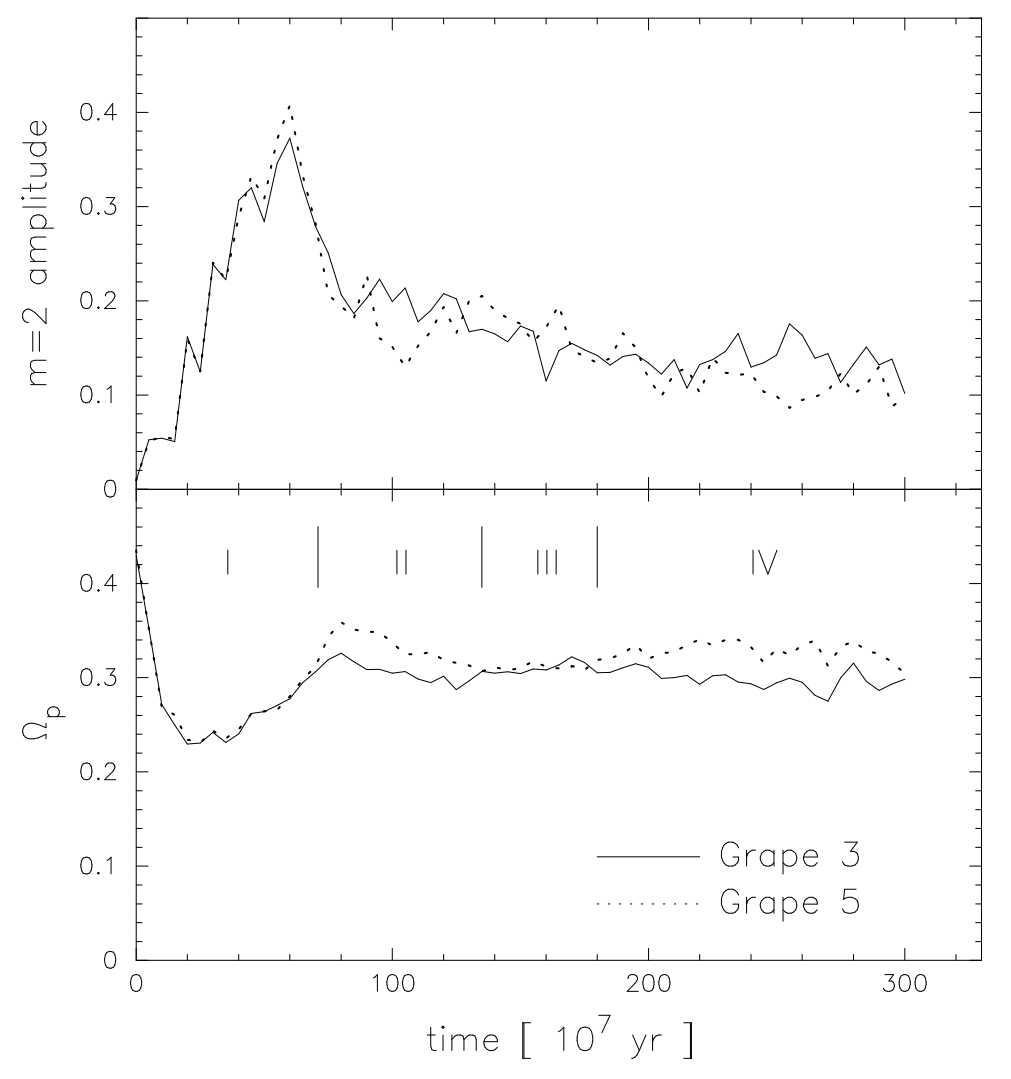

Figure 7.1: Direct comparison of the GRAPE-3 and GRAPE-5 results of the reference model I0. The upper and lower panel show the bar strength and the pattern speed $\Omega_{\mathrm{p}}$, respectively, as a function of time for the GRAPE-3 (full line) and GRAPE-5 (dotted line) simulations. In the lower panel we mark the four phases of $\Omega_{\mathrm{p}}$ as defined in the text.

(I) $20<t \leq 70$ : During the initial growth phase of the bar, $\Omega_{\mathrm{p}}$ increases from 0.23 to roughly 0.3 . During this first phase the pattern speed is the same in both simulations.

(II) $70<t \leq 135$ : In the second phase, the pattern speed in both simulations first increases, reaching a local maximum of $\Omega_{\mathrm{p}} \approx 0.4$ at roughly $t=80$ and decreases again afterwards. In the G5 simulation, however, $\Omega_{\mathrm{p}}$ is temporarily about 12 per cent higher than in the G3 run.

(III) $135<t \leq 180$ : In the third phase, the pattern speed in both runs is once again identical, remaining at a constant level of some $\Omega_{\mathrm{p}}=0.3$.

(IV) $180<t \leq 300$ : While $\Omega_{\mathrm{p}}$ remains roughly constant in the G3 simulation, it increases again in the G5 run. The pattern speed in G5 is, as in phase II, about 
12 per cent higher than in G3.

A possible explanation for the differences could be the higher numerical accuracy of the G5. We will show, however, that the different evolution cannot be attributed solely to this and some other numerical effect(s) must also play a role.

\subsubsection{Switching machines}

In a first test we check, if the different evolution of $\Omega_{\mathrm{p}}$ on the two machines is sensitive to the evolutionary phases of the bar. We therefore use different snapshots from the G5 run to restart the simulation on the G3. The restart times (hereafter $t_{\mathrm{rst}}$ ) have been chosen from the different phases of $\Omega_{\mathrm{p}}$ as described in the previous section.

We show the evolution of the pattern speed in Fig. 7.2. In the run restarted in the last phase IV, i.e. at $\left(t_{\mathrm{rst}}=220\right.$; upper panel $)$, the pattern speed coincides with the G5 result till the end of the run. This result may give some indication that the underlying dynamics of the disc has been changed in an earlier phase of evolution and further evolution is not dependent on the hardware used. On the other hand, the integration period might have been too short for numerical effects introduced from the G3 to take place. Restarting the model in phase III, i.e. at $t_{\mathrm{rst}}=160$ (middle panel), the evolution of $\Omega_{\mathrm{p}}$ is similar as in the G5 case, i.e. the pattern speed increases in phase IV and follows the G5 result afterwards. There is some indication, however, that $\Omega_{\mathrm{p}}$ decreases somewhat towards the end of the run. Finally, restarting the model in phase I $\left(t_{\mathrm{rst}}=60\right.$; lower panel), we find an increment of $\Omega_{\mathrm{p}}$ in phase II, but later on $\Omega_{\mathrm{p}}$ settles down somewhere between the G3 and G5 results. To summarize, we find a general trend, that the restarted simulations on the G3 tend to stay closer to the G5 result. Thus if the underlying dynamics of the disc is really affected by the type of hardware used, this must have occurred in an early phase, i.e. during the bar-growth and/or gas-inflow phase.

\subsubsection{Hybrid tree/GRAPE codes}

Besides the gravitational force and potential calculation, the GRAPE hardware returns an index array containing all particle neighbours within a sphere with radius $1.3 \times 2 h_{i}$, where $h_{i}$ is the SPH smoothing length, around a given particle position $\mathbf{x}_{i}$. This 30 per cent buffer is required when using multiple time-steps, because of the symmetric nature of the kernel evaluation. However, the size of the buffer zone is based on tests which show that it will find the majority of the required neighbours, but is not guaranteed to locate them all. In contrast the software method we use will find all the neighbours within the truncation error of the machine.

To see if the difference in the neighbour search method is effecting the pattern speed, we replace in the code both tasks, i.e. the force calculation and the SPH particle neighbour-list search, separately by the optional treecode routines. The opening 


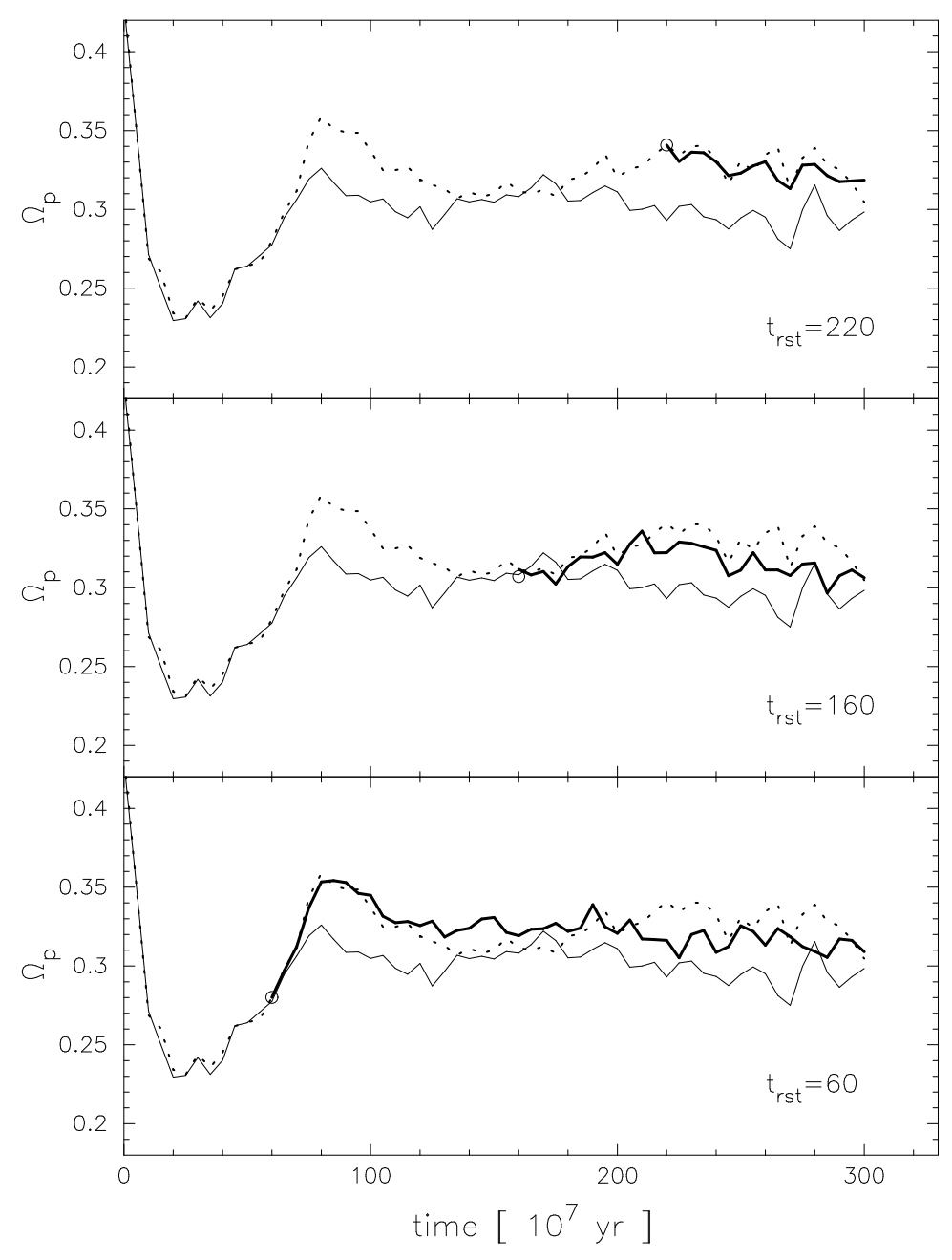

Figure 7.2: Pattern speed of the bar in GRAPE-3 models (thick full line), which have been restarted from GRAPE-5 snapshots. The G3 and G5 results are shown for comparison with a thin full line and dotted line, respectively. The restart time $t_{r s t}$ is given in each panel.

angle $\theta$ used in the treecode has been chosen as $\theta=0.2$ in the force calculation. The results are shown in Fig. 7.3. Using the treecode force calculation (upper panel), the evolution of $\Omega_{\mathrm{p}}$ is similar to the pure G3 simulation in all four phases. We find differences in the evolution, however, when using the software-based neighbour search (Fig. 7.3; lower panel). In the beginning $\Omega_{\mathrm{p}}$ matches with the pure G3 run but then lies somewhat between G3 and G5. In the phases III and IV algorithm. These results may indicate that the diverging evolution is in part attributable to the neighbour search method. However, using a brute force algorithm, we find that all lists have most of the neighbours in common, within some 1 per cent. Further tests, 


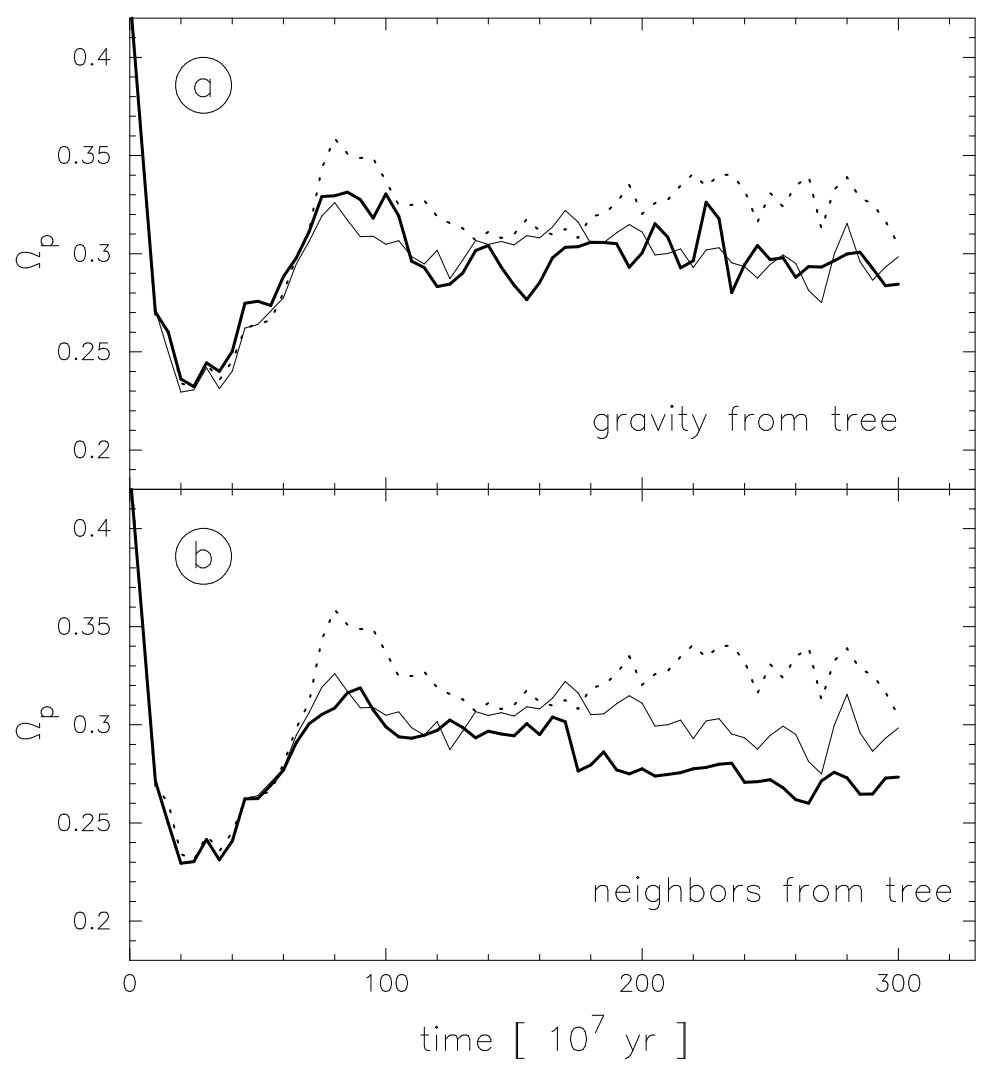

Figure 7.3: Results of the hybrid Tree-GRAPE3-code. Panel (a) and (b) show the pattern speed of the runs in which the force calculation and the neighbour search, respectively, has been done by the treecode on the front-end machine.

in which we increase the size of the buffer zone to $1.5 \times 2 h$ give similar results. The outcome of these tests shows that even small variations in the neighbour-lists make a difference in the evolution of the bar pattern speed

\subsubsection{Hydrodynamics}

To check the numerical accuracy of the hydrodynamical routines in our code in more detail, we run a set of simulations of the collision between two non-gravitating isothermal sheets of gas (see, for example, Heller \& Shlosman 1994; see also Appendix 7.11), using the G3, G5 and the treecode. In this test, the SPH particles are non-self-gravitating and thus the gravitational force calculation does not play a role. The particles are initially located on a grid with a spacing of $\mathrm{d} x=\mathrm{d} y=0.03$ units and with equal masses of value $m=p \mathrm{~d} x \mathrm{~d} y=1 \mathrm{~d} x \mathrm{~d} y$. Particles located at $x<0$ have an initial velocity of $v_{0}=1$, i.e. Mach 1 , while those with $x>0$ have $v_{0}=-1$. The shock profiles of the G3, G5 and treecode runs in comparison with the analytic 


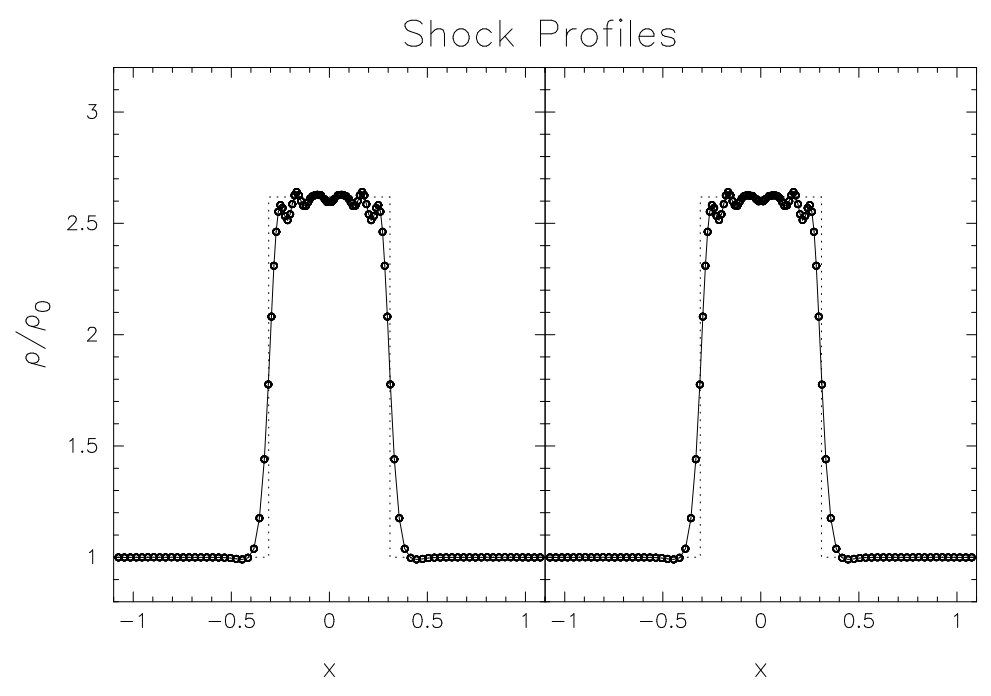

Figure 7.4: Shock profile of two colliding isothermal sheets of gas. The left- and right-hand panel show the normalized density profiles produced by the treecode and GRAPE-5, respectively, both marked by points. The analytic solution (dotted line) and the result of GRAPE-3 (full line) are shown for comparison in both panels.

solution are shown in Fig. 7.4. We find that the SPH algorithm adequately reproduces the analytic density profile of the Mach 2 shock at time $\tau=0.5$. Furthermore, the three different runs do not show any discrepancies.

\subsection{Systematic numerical tests}

Since the GRAPE-5 hardware is about ten times more accurate in the force calculation than GRAPE-3 (Kawai et al. 2000), we consider the numerical accuracy as an possible source of error in the deviating evolution of $\Omega_{\mathrm{p}}$. Since the force calculation is hardwired on the GRAPE the numerical accuracy is the same for all simulations on this hardware. In the following sections we will therefore present a set of treecode simulations using ordinary workstations, applying different opening angles $\theta$ in order to look for a possible correlation between the evolution of $\Omega_{\mathrm{p}}$ and the accuracy of the force calculation. In order to make a comparison feasible, we first calculate the relative errors from the treecode and the GRAPE simulations (Sec. 7.4.1). Furthermore, in order to have a larger sample of simulations available, we set-up three more realisations of our reference model. The new realisations have been constructed by changing the initial $(x, y)$ component of both the position and velocities of all particles, keeping the particles initial radius fixed and rotating it by a random angle about the $z$-axis. The angle of rotation for each has been determined using a random number generator with three different initial seeds. All 


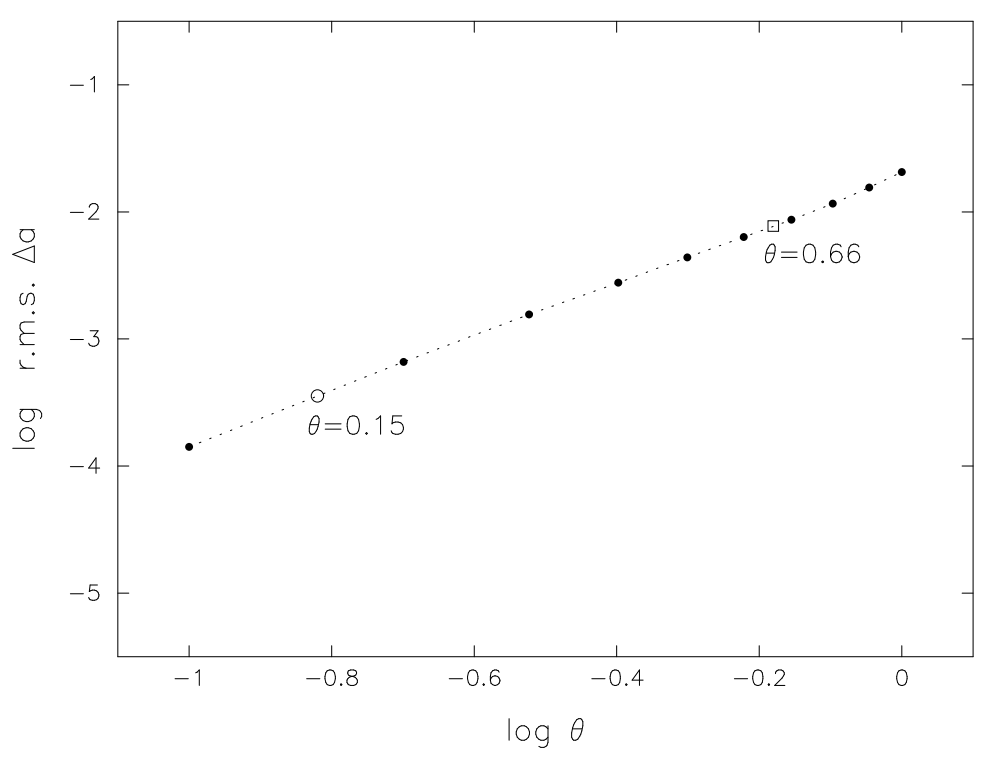

Figure 7.5: Logarithmic root-mean-square (r.m.s.) relative errors in acceleration, $\left\langle\|\Delta \mathbf{a}\|^{2} /\|\mathbf{a}\|^{2}\right\rangle^{1 / 2}$. We show the results of the treecode (full circles), GRAPE-5 (open circle) and GRAPE-3 (open square). For the GRAPE results we give the corresponding opening angle for the treecode.

models have been run on the GRAPE-3 and with the treecode, using opening angles $\theta=0.1,0.2, \ldots, 1.0$. The results are discussed in Sec. 7.4.2.

\subsubsection{Accuracy in the force calculations}

The accuracy of the force calculation in treecode simulations is determined by the opening angle $\theta$. In order to provide a comparison between the GRAPE and the treecode simulations, we first compare the accuracy in the force calculation. Therefore we select a snapshot ( $t=100$ ) from the G5 simulation of model I0 and calculate the total gravitational forces for the stellar disc particles using a direct summation method, giving us a reference force $\mathbf{a}_{0}$, where $\mathbf{a}_{0}=\sum_{i=1}^{N} a_{i}$. Then we repeat the force calculation for the same snapshot using the G3, G5 and the treecode with opening angles $\theta=0.1,0.2, \ldots, 1.0$. We than calculate the root-mean-square (r.m.s.) errors of the forces, as defined by:

$$
\sigma_{\Delta a}=\left(\frac{\left|\mathbf{a}-\mathbf{a}_{0}\right|^{2}}{\left|\mathbf{a}_{0}\right|^{2}}\right)^{1 / 2}
$$

We show the results in Fig. 7.5, where we plot the logarithmic r.m.s. relative errors as a function of $\log \theta$. We find that the error in the force calculation increases 


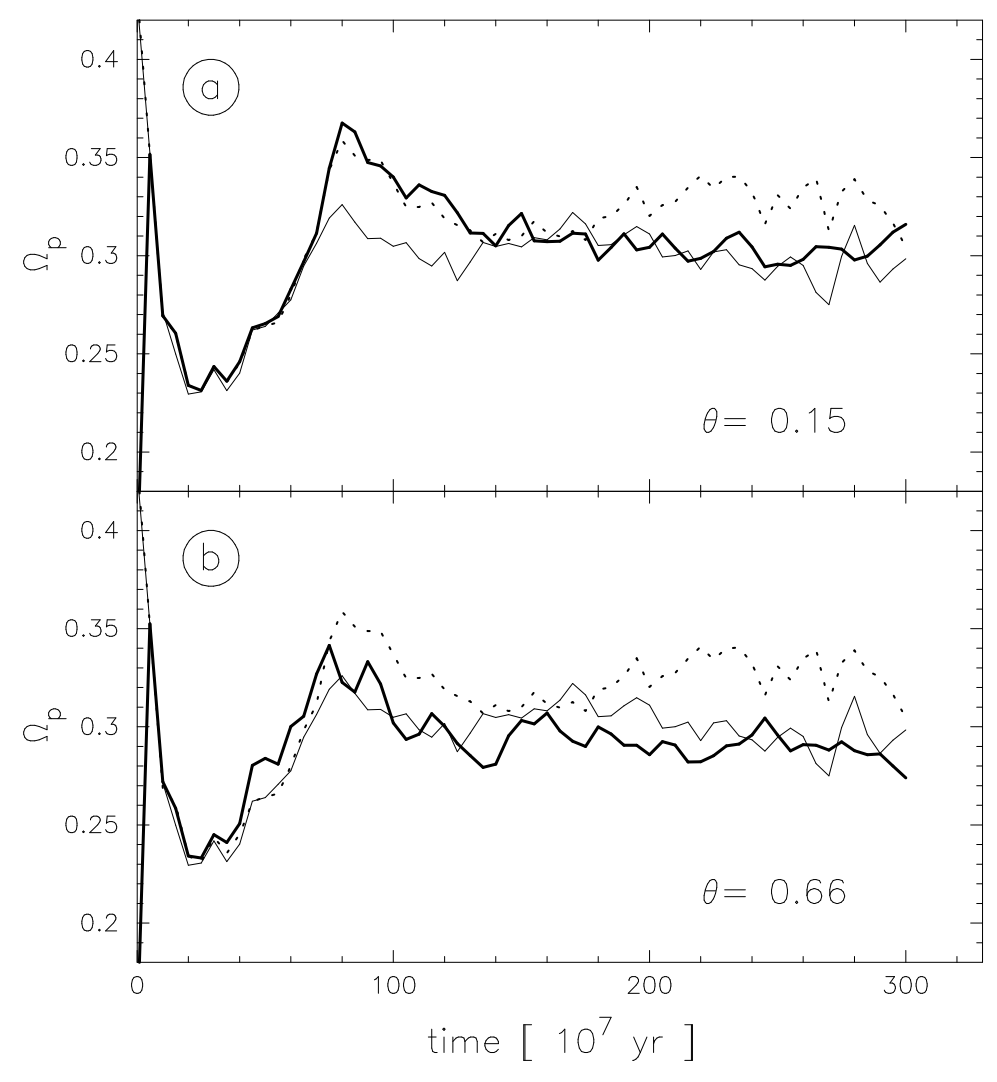

Figure 7.6: Comparison of the treecode models with the deduced opening angles for G5 ( $\theta=0.15$, upper panel) and G3 $(\theta=0.66$, lower panel).

linearly in this plot, which is in agreement with the results of Barnes \& Hut (1989). From our graph we can now look up the $\theta$ corresponding to the accuracy of G3 and G5, which gives $\theta=0.66$ and 0.15 , respectively. In order to check this prediction, we run two treecode simulations of our reference model with these opening angles and compare the results with the ones from G3 and G5 (see Fig. 7.6). We find that the simulation with $\theta=0.66$ fairly reproduces the $\mathrm{G} 3$ result of our reference model. The simulation with $\theta=0.15$, however, does not reproduce the G5 result as expected, though in the early phase (up to $t \approx 120$ ) it shows a similar evolution.

\subsubsection{Treecode with different opening angles}

In this section we describe a set of simulations using the treecode with opening angles $\theta=0.1,0.2, \ldots, 1.0$ in order to study the influence of the accuracy of the force calculation on the evolution of $\Omega_{\mathrm{p}}$. We create three more model realisations based on our reference model I0, following the description in Sec. 7.2.3, to further check the influence of the intrinsic numerical noise in the initial conditions. For the 


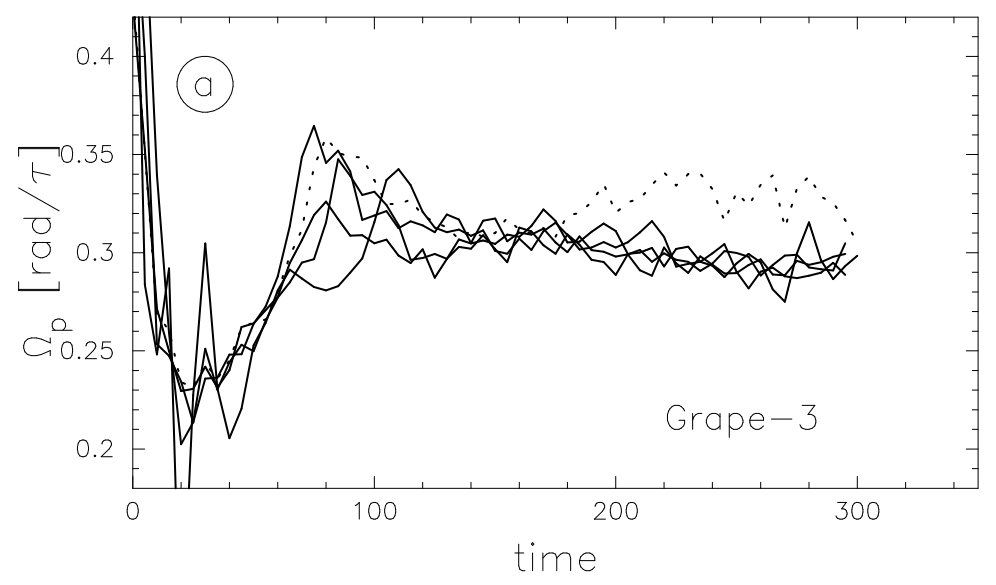

Figure 7.7: Model with different initial positions and velocities using different initial random seeds. The figure shows the results of the GRAPE-3 simulations. The reference model from GRAPE-3 and GRAPE-5 are shown for comparison.

latter, we first run simulations using the GRAPE-3 hardware with the new models I1, I2 and I3. In Fig. 7.7 we show the bar's pattern speed as a function of time in these models. We find some deviations in pattern speed in the early phases of evolution (i.e., phase I and II), but then $\Omega_{\mathrm{p}}$ converges at later times to a pattern speed which is smaller than in the G5 run of model I0. Thus the change in the particles initial positions results in a different evolution of the pattern speed.

In Fig. 7.8 we show the pattern speed of the four models, using this time the treecode with different opening angles $\theta=0.1,0.2, \ldots, 1.0$. As can be seen in this plot, the scattering of $\Omega_{\mathrm{p}}$ between the four realisations increases in simulations with a lower accuracy in the force calculation, i.e. with increasing $\theta$. In order to quantify this result, we calculate the mean deviation of $\Omega_{\mathrm{p}}$ for the simulations in the four phases $k=\mathrm{I}, \ldots, \mathrm{IV}$, as defined in Sec. 7.3. We therefore define the mean deviation as:

$$
\sigma_{k}=\frac{1}{2 \Delta t_{k}} \sum_{i, j=0}^{3}\left|\Omega_{\mathrm{p}}^{i}-\Omega_{\mathrm{p}}^{j}\right|_{k}
$$

where $\Delta t_{j}$ is the time interval of each phase. Furthermore we calculate the timeintegrated deviation as:

$$
\sigma_{\mathrm{T}}=\sigma_{\mathrm{I}}+\sigma_{\mathrm{II}}+\sigma_{\mathrm{III}}+\sigma_{\mathrm{IV}}
$$

We use the corresponding definitions for calculating the mean deviation of the bar's amplitude. In Figs 7.9 and 7.10 we show $\sigma_{j}$ and $\sigma_{T}$ of the simulations with different $\theta$. While simulations with $\theta \leq 0.5$ have relatively low deviations, which is roughly 

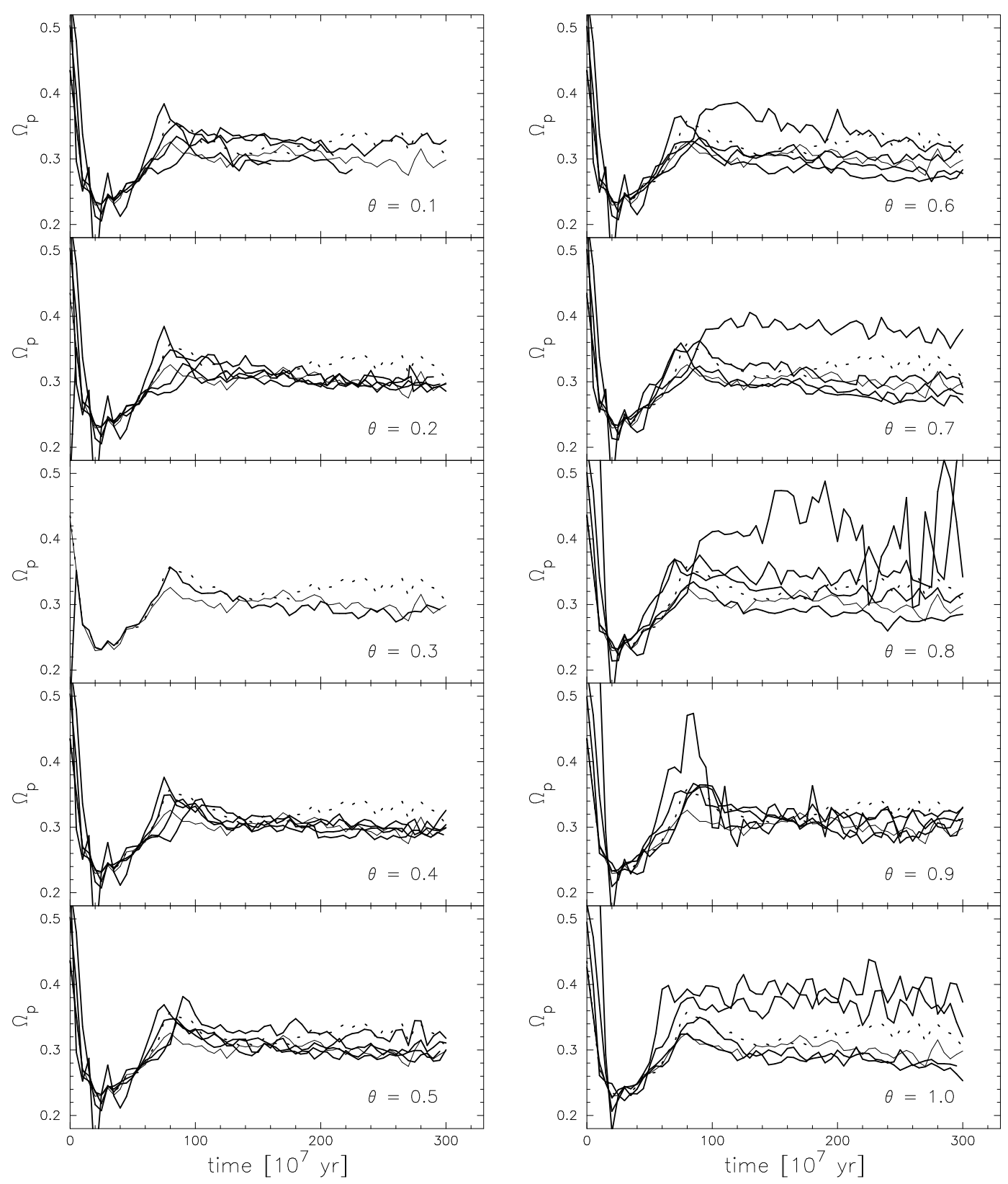

Figure 7.8: Pattern speed of the four model realisations as a function of time. The simulations have been run with the treecode using different opening angles as given in the lower right of each panel. The results of the GRAPE-3 and GRAPE-5 reference model are plotted by a thin full line and a dotted line, respectively.

constant for those runs, the deviation for larger $\theta$ starts to increase approximately linearly. Assuming a gaussian-like distribution of the values of $\Omega_{\mathrm{p}}$, the errors with 


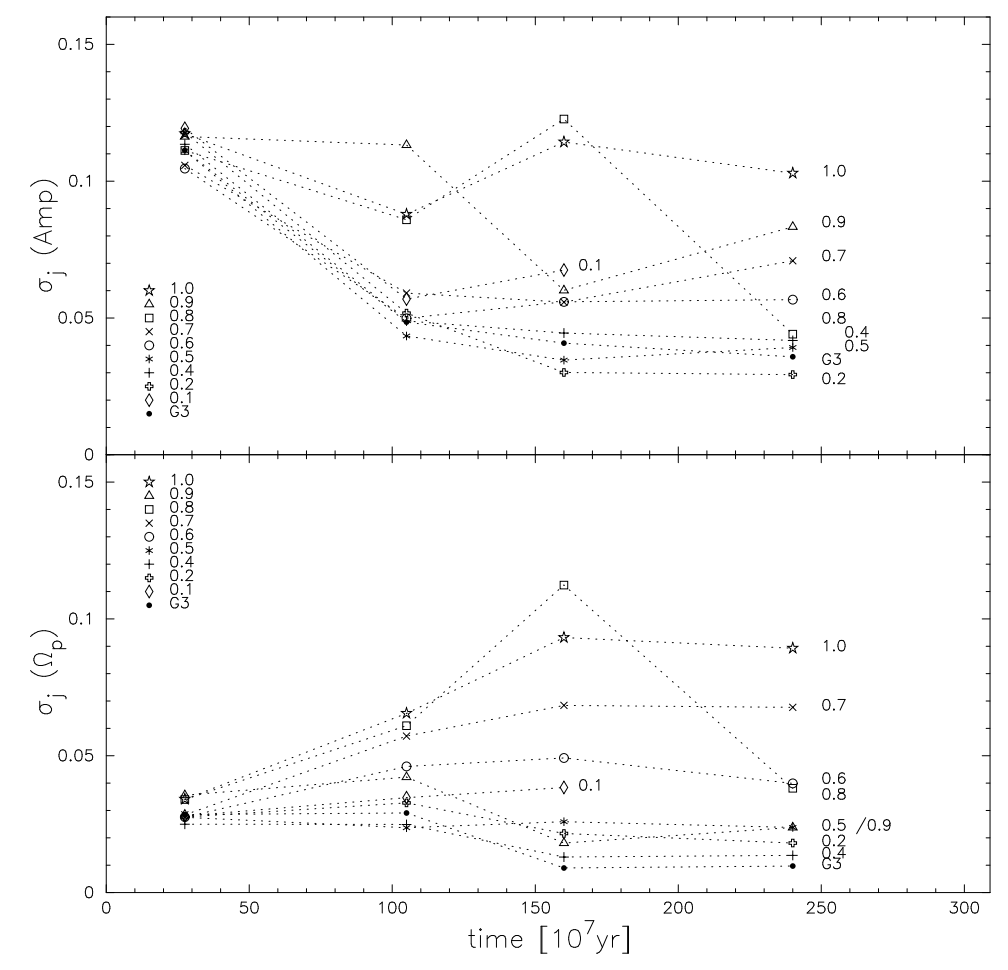

Figure 7.9: Mean deviation $\sigma_{j}$ of the bar strength (upper panel) and pattern speed (lower panel) in each phase (as defined in the text) for the different model realisations. Simulations with different opening angles are marked by the different symbols as given in the plot.

respect to the true gaussian average should be proportional to the width of the gaussian. Thus the average of the results of the large $\theta$ simulations gives a much poorer estimate of the true average than the corresponding one for a small $\theta$. For an equal accuracy, more realisations for large $\theta$ would be required. This might explain the off-set result for the models with $\theta=0.9$ in Fig. 7.10.

\subsection{Further numerical tests}

\subsubsection{Treecode with and without quadrupole terms}

The treecode simulations presented so far in this work have been neglecting the quadrupole terms in the force calculation. We thus run a test with our reference model I0 using the treecode with an opening angle of $\theta=0.7$, taking the quadrupole moment into account. In Fig. 7.11 we show a comparison of the runs with and without quadrupole terms. We find that the higher accuracy in the force calculation 


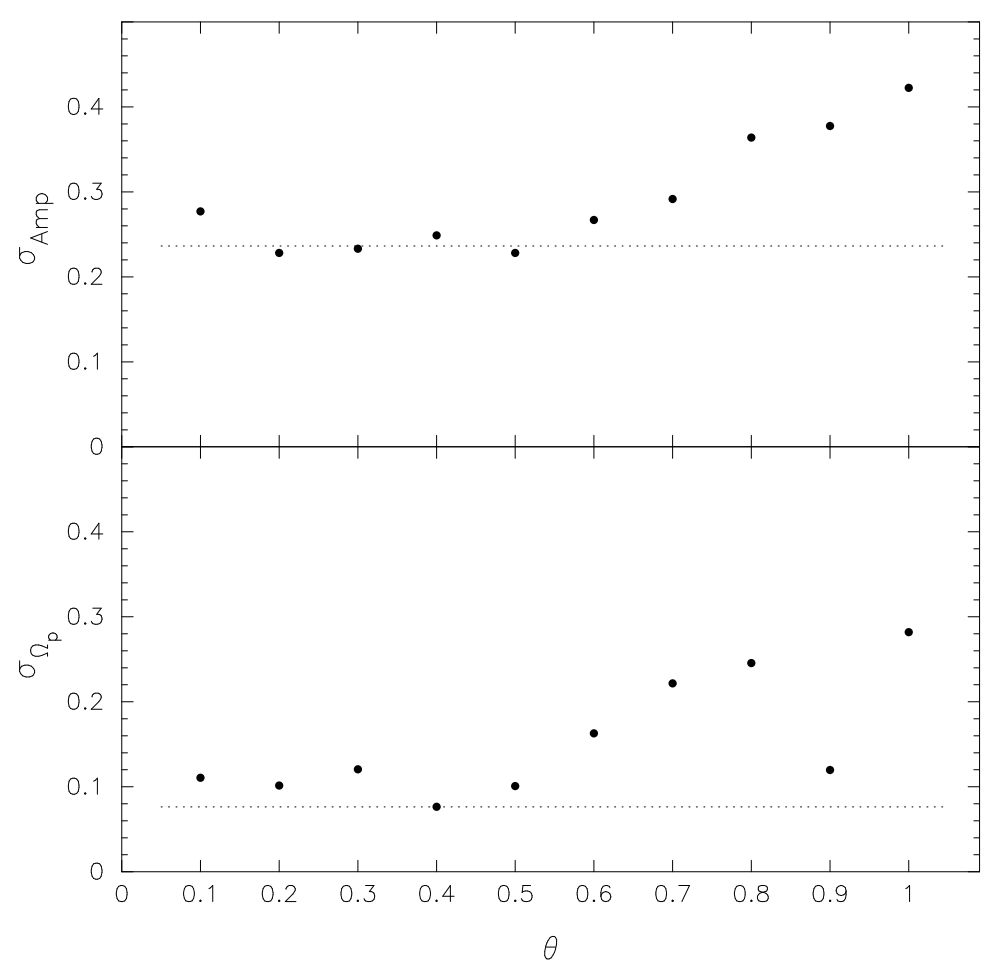

Figure 7.10: Time-integrated deviations $\sigma_{T}$ of the bar strength (upper panel) and pattern speed (lower panel) integrated over the four phases as a function of the opening angle. The deviation from the GRAPE-3 simulations is given by the horizontal dotted line.

(with quadrupole terms) results in a different evolution of $\Omega_{\mathrm{p}}$, being closer to the G5 result. This results is in agreement with the fact, that the evolution of the bar is sensitive to the accuracy of the force calculation.

\subsubsection{Treecode with different time-step limits}

The time stepping affects the results mainly in terms of energy conservation. We run different simulations with the treecode and GRAPE-3, varying the maximum and minimum system time-steps, $\tau_{\max }$ and $\tau_{\min }$, respectively. Fig. 7.12 shows the pattern speed of two runs with different maximum system time-steps using the treecode. We do not find any significant differences between the two models, although the pattern speed in the model with $\tau_{\max }=0.05$ seems to increase slightly again at the end of the run. We also checked if the minimum time-step affects the pattern speed in the simulations. The results of these runs are shown in Fig. 7.13. 


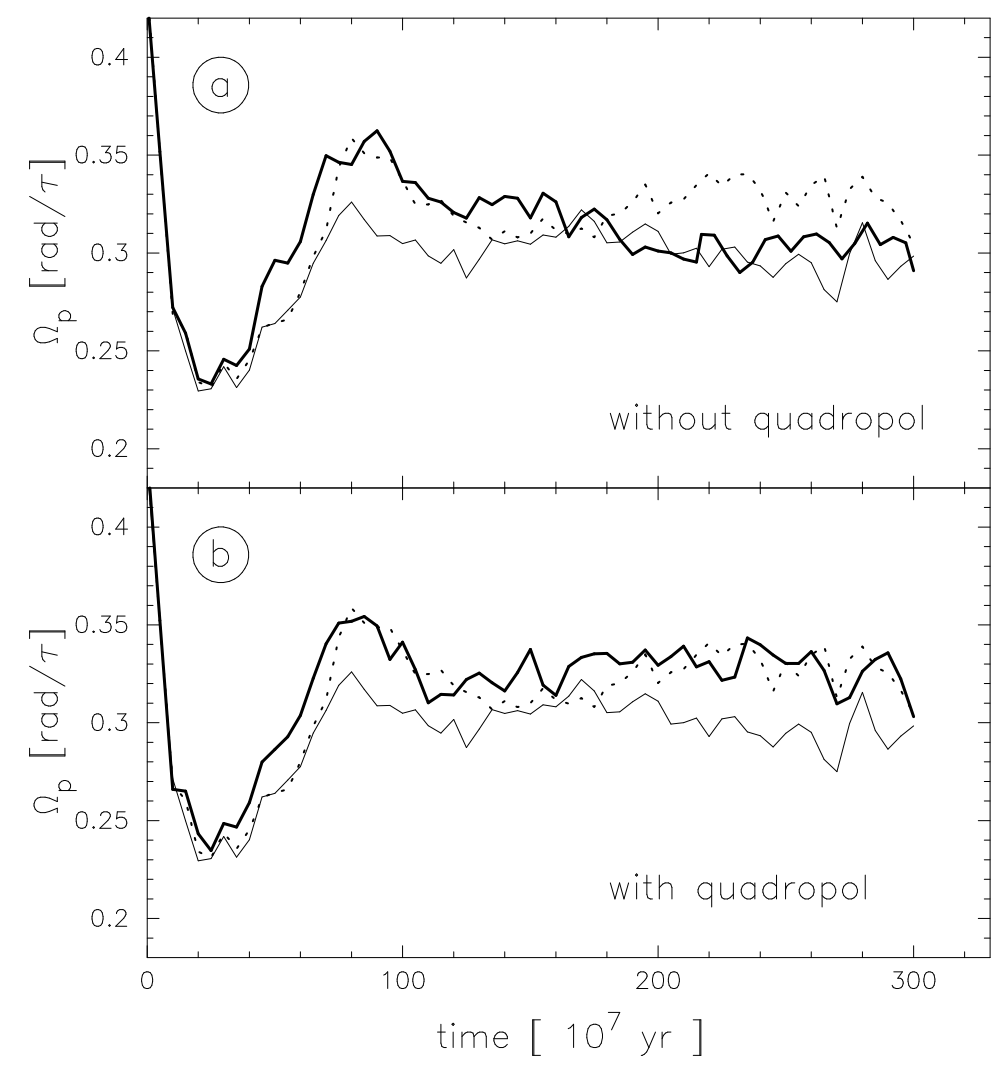

Figure 7.11: Comparison of treecode simulations of model I0 with $\theta=0.7$. Panels (a) and (b) show the results without and with quadrupole terms in the force calculation, respectively. The results of the G3 (dashed line) and G5 (dotted line) runs are shown for comparison.

\subsection{Properties of the bars}

As we have shown in Sec. 7.4.2, the evolution of the disc is affected by the intrinsic numerical noise in the $N$-body/SPH model realisation. So far we have mainly concentrated on the evolution of the bar's pattern speed with respect to the numerical accuracy of the code and the hardware. In this section we now discuss the properties of the bar and the disc in general.

In Fig. 7.14 we show the bar strength and the pattern speed as a function of time, along with the logarithmic surface density of the gas (roughly within the inner kpc) at the end of the simulations as a function of radius. We have arranged the plot such as to compare simulations with different opening angles. As can be seen in the plot, both the bar strength and the pattern speed show a different evolution for the same model. We note that the deviation of the three quantities is minimum for model I3. This indicates that the variations in $\Omega_{\mathrm{p}}$ depend on both the opening angle and the 


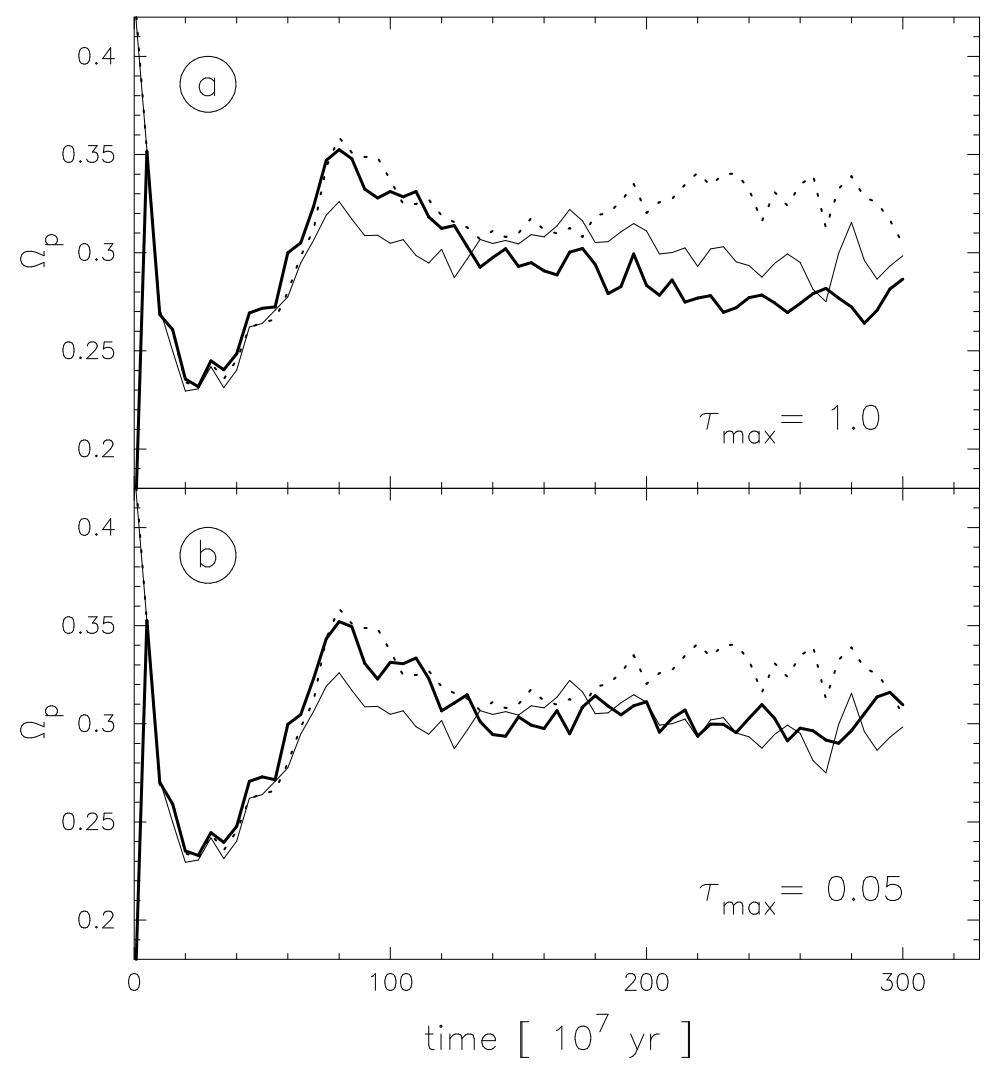

Figure 7.12: Treecode with an opening angle of $\theta=0.5$ and different maximum system time-steps $\tau_{\text {max }}$. Panel (a) and (b) show the pattern speed for runs with $\tau_{\max }=1.0$ and $\tau_{\max }=0.05$, respectively (full lines). The results of GRAPE-3 (dashed line) and GRAPE-5 (dotted line) are shown for comparison in both panels.

intrinsic noise of the model.

Models which show strong deviation from the mean evolution also show conspicuous differences in the central gas mass distribution. As can be seen from Fig. 7.8, both the pattern speed and the bar strength stay roughly constant approximately after $t=150$ in most of the simulations. We therefore calculate the average of $\Omega_{\mathrm{p}}$ and $S_{\mathrm{B}}$ from $t=150$ till the end of the run for each simulation. Furthermore we calculate the central gas mass within a radius $R=0.3$, which is just outside the circumnuclear gaseous disc formed in the models. The results are then plotted versus the opening angle $\theta$ in Fig. 7.15. From this plot, however, we cannot find an obvious correlation between the central gas mass and the evolution of the bar. Thus the evolution of the bar seems to be dependent on the density profile of the gas (or vice versa) rather than on the absolute amount of gas in the central region of the disc. We do find a roughly linear correlation between the central gas mass and the pattern speed of the bar, as shown in Fig. 7.16. 

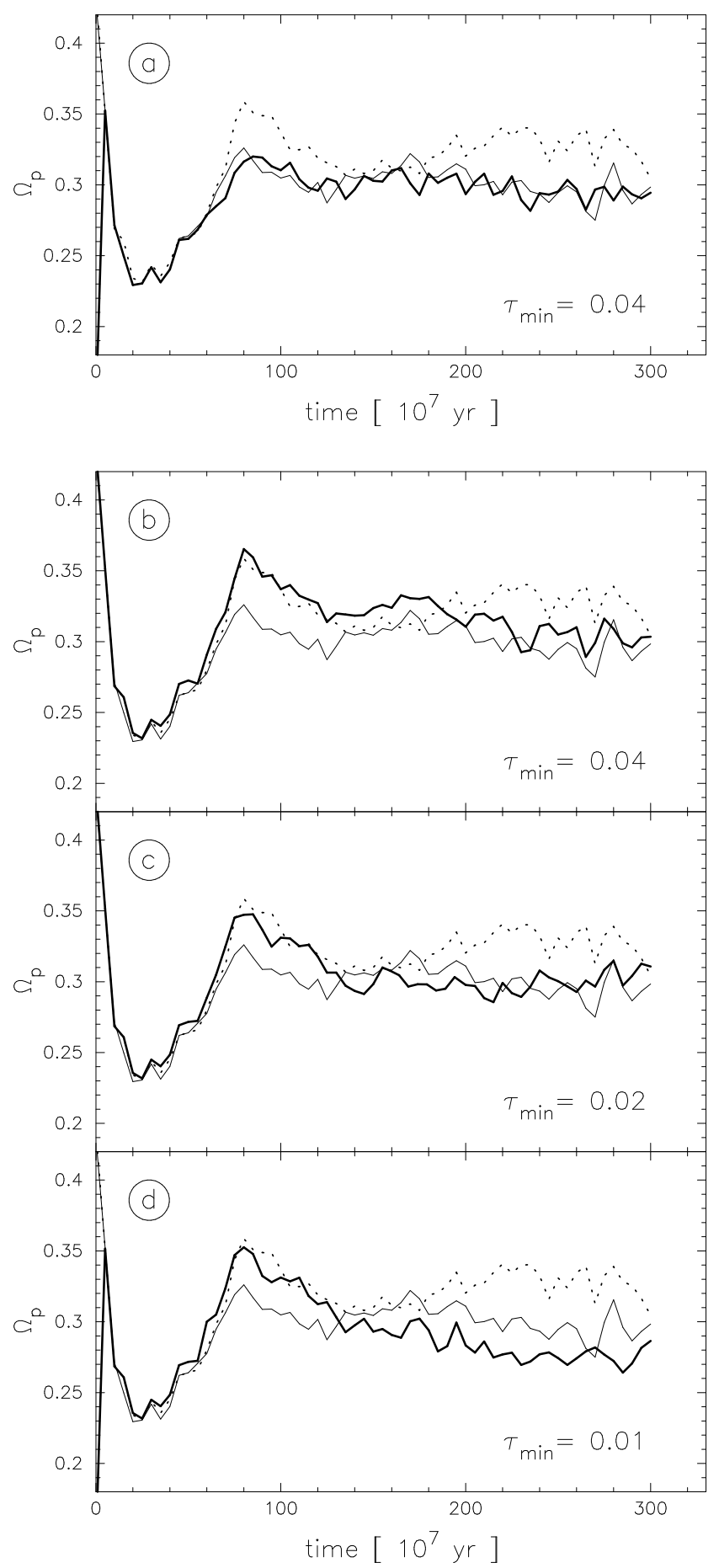

Figure 7.13: Models with different minimum system time-steps $\tau_{\min }$. The panels (a) to (d) shows the result of the GRAPE-3 and the treecode simulations (with $\theta=$ $0.5)$, respectively. The minimum system time-steps $\left(\tau_{\min }=0.04,0.02\right.$ and 0.01$)$ used in the runs are given in the lower right of each panel. The results of the default GRAPE-3 and GRAPE-5 models are shown with a thin full line and dotted line, respectively, for comparison. 


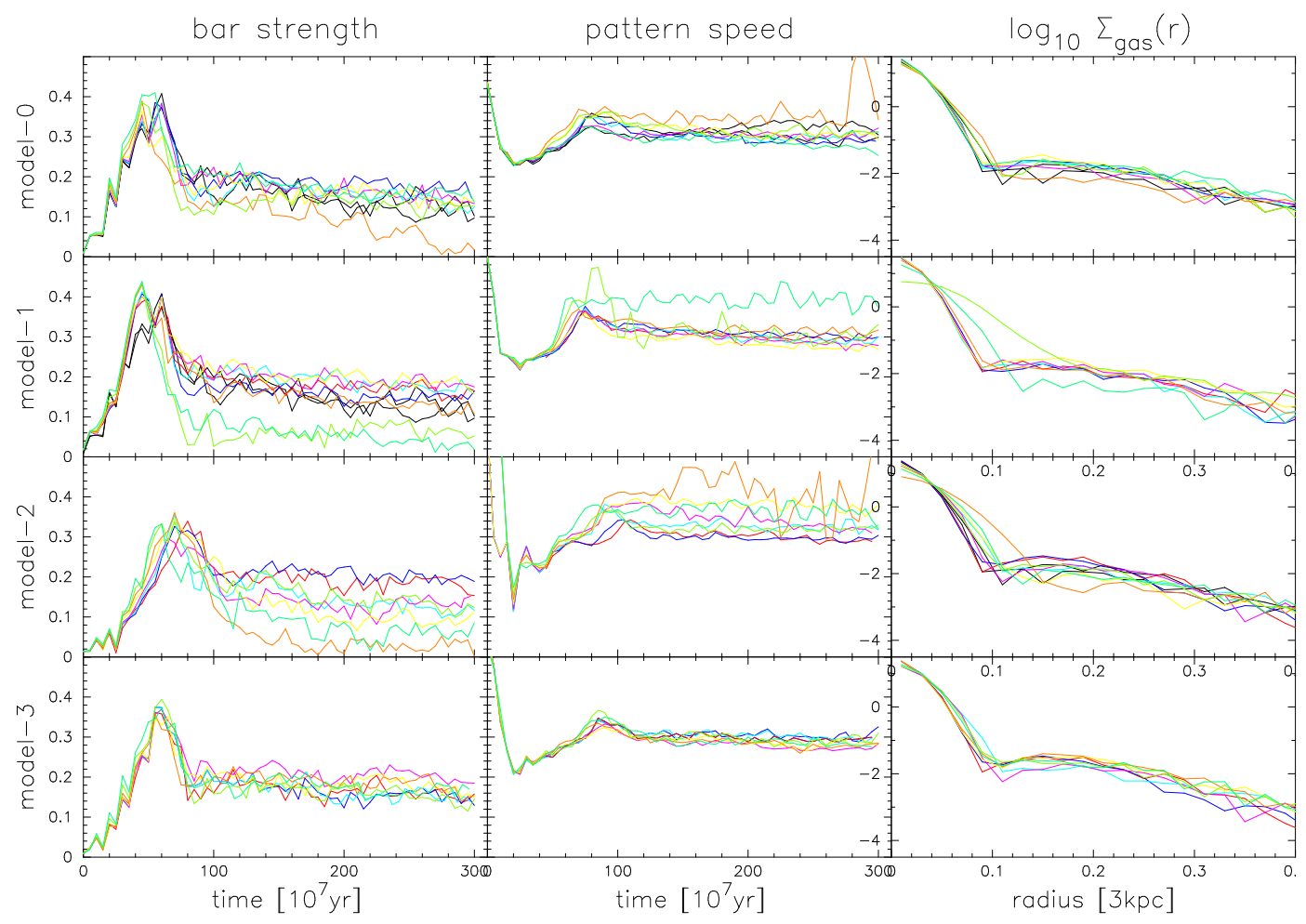

Figure 7.14: Bar strength, pattern speed and logarithmic surface density for the models with different initial seeds. The different colors represent simulations with different opening angles $\theta(0.4,0.5, \ldots 1.0)$. Lower theta runs are not yet included. Also the y-axis labels for the density have to go to the other side.

Athanassoula (2003) found, using pure $N$-body simulations, a correlation between the bar strength and the pattern speed of bars in isolated disc galaxies. The author argues, that the evolution of bars in isolated disc galaxies is driven by the redistribution of angular momentum and that the strength and the pattern speed of the bar are determined by the amount of angular momentum exchanged. The main difference between her models and the one presented in this work is that we have an additional gas component in the disc. The gas can dissipate energy and loose angular momentum in the inner regions. In order to compare the properties of the bars formed in our models to the ones in the purely stellar models, we first calibrate our model units appropriately, as proposed in Athanassoula \& Misiriotis (2002). We then compare them to the ones in Athanassoula (2003). The result is shown in Fig. $7.17^{2}$. We find a similar correlation between the bar strength and the pattern

\footnotetext{
${ }^{2}$ Care is needed when evaluating the plot, because the results of Athanassoula (2003) represent different models, while our results are based on four realisations of the same model with different numerical accuracy.
} 


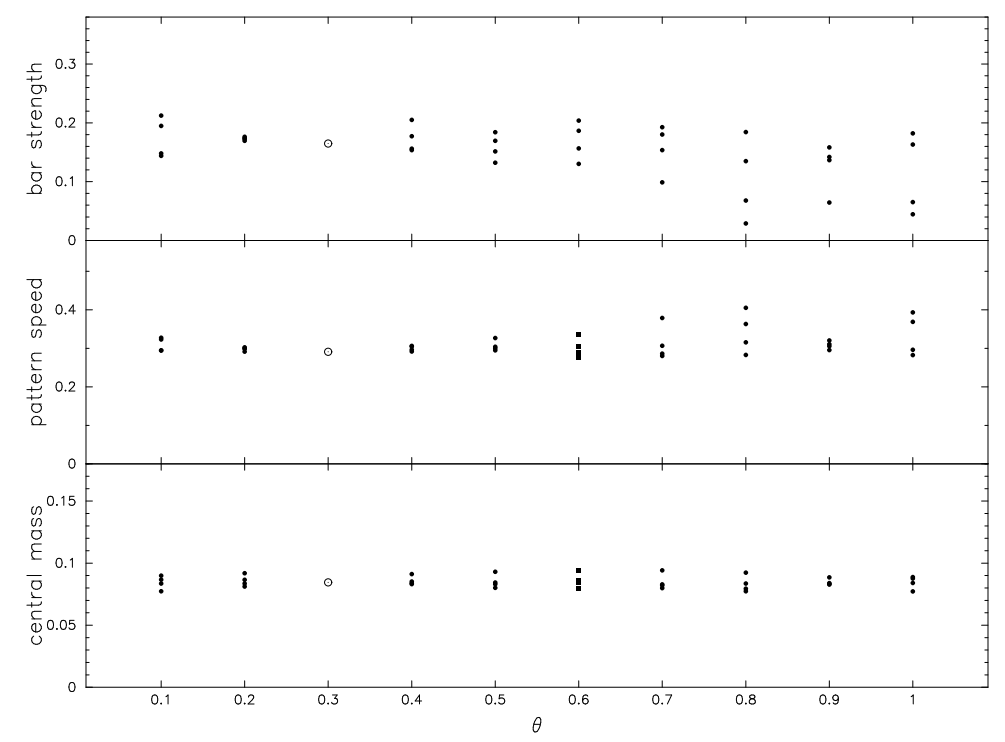

Figure 7.15: Bar strength, pattern speed and central gas mass (within $R=0.2$ ) for the four realisations versus the opening angle used in the treecode simulations. Note that for $\theta=0.3$ only one model has been run and so only a single point representing it is shown.

speed, which is, however, slightly off-set from the one in the purely stellar models. We associate this difference with the presence of the dissipative component in our models.

\subsection{Discussion}

The set of simulations presented in this work shows that the pattern speed of the bar can show variations of up to 13 per cent between the different runs. These variations are due to more than one numerical effect, which are difficult to disentangle.

There is the intrinsic numerical noise in the models, which can result in a different evolution of the bar (Figs 7.7 and 7.14). Because of the large number of simulations presented in this work, we used a relatively low number of particles in our models ${ }^{3}$. This discreteness effect can probably be significantly reduced in models with larger $N$. Thus the value of larger $N$ models for even basic studies of disc evolution is clear.

There are also effects which are directly related to the numerical accuracy of the code and the hardware used. One aspect of this is the accuracy of the force calculation in the simulations, because it directly affects the momentum conservation

\footnotetext{
${ }^{3}$ The simulations presented in this work took several week/month on modern work-stations.
} 


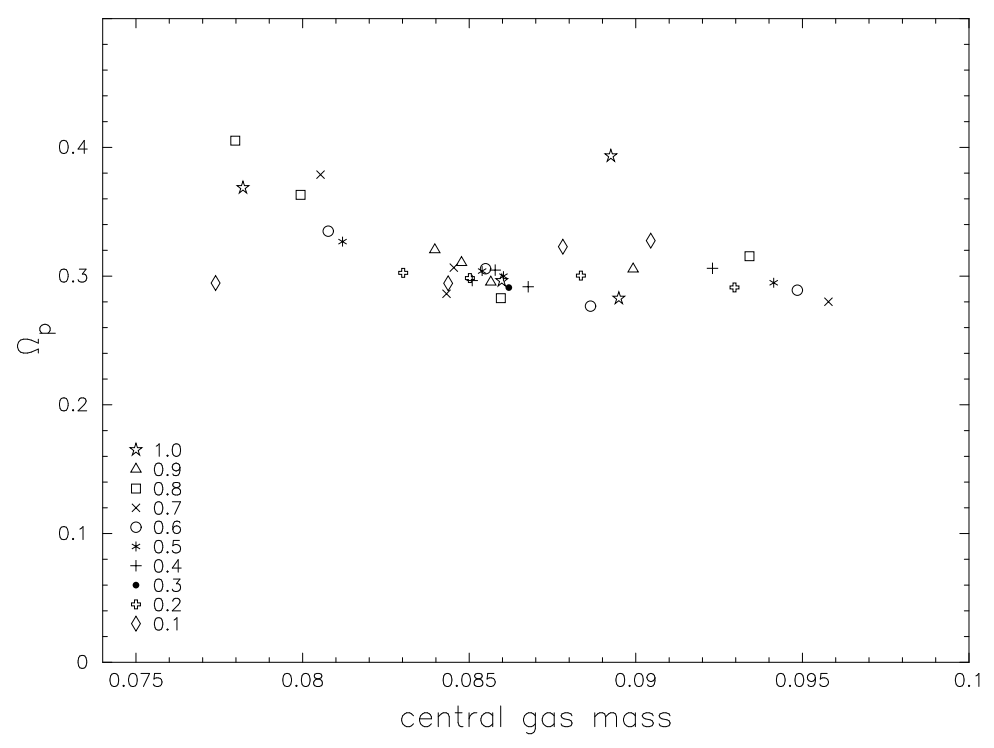

Figure 7.16: Pattern speed versus central gas mass (within $R<0.3$ ) at the end of each run. Sets of models with different initial seeds are represented by black squares, red stars, blue triangles and green circles. The corresponding GRAPE simulations are represented by open circles with a cross (GRAPE-3) and a dot (GRAPE5). Opening angles are given for some of the simulations below the corresponding symbols.

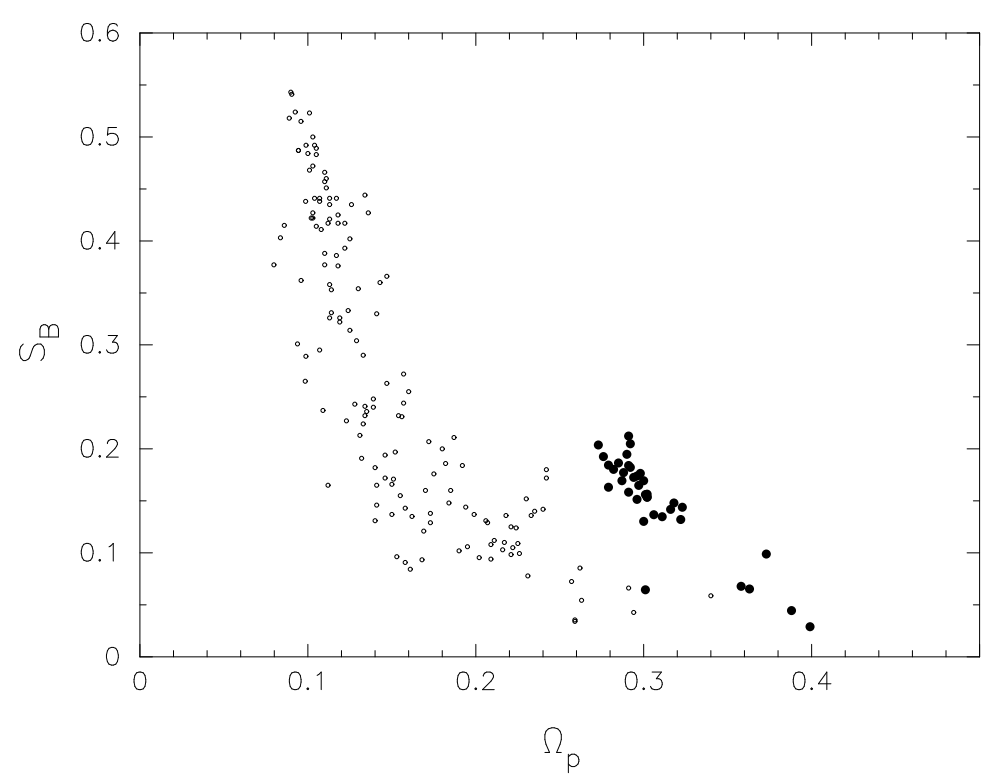

Figure 7.17: Bar strength $S_{\mathrm{B}}$ versus pattern speed $\Omega_{\mathrm{p}}$ in units used by Athanassoula $\&$ Misiriotis (2002). The dots and bullets show the results of the isolated models of Athanassoula (2003) and of our four model realisations using the treecode with different opening angles. 
in the models. For the GRAPE hardware, which is often used for galactic and cosmological simulation, the accuracy is fixed by the hardware itself. The GRAPE-5 is about ten times more accurate than its progenitor GRAPE-3 (see Fig. 7.5). This difference alone shows a clear impact on the evolution of the bar's pattern speed (Fig. 7.1) in our models. The deviation of $\Omega_{\mathrm{p}}$ also shows a clear correlation with the numerical accuracy when using the treecode (Fig. 7.10), in which we can vary the accuracy of the force calculation by means of the opening angle $\theta$. The results are in agreement with the assumption of a gaussian-like distribution for the deviations in $\Omega_{\mathrm{p}}$. This is however based on only four different models and additional simulations will be required to fully test this hypothesis. The deviation of $\Omega_{\mathrm{p}}$ in the different simulations increases linearly for opening angles $\theta \geq 0.5$, and thus a relatively small $\theta$ is required to minimize the influence of the force calculation. Another approach to increase the numerical accuracy is to take the quadrupole terms in the force calculation of the treecode into account. So far, we have only one simulation of this kind available, but the result is in agreement with our previous finding that the force accuracy strongly affects the evolution of the bar.

Another aspect is the time-stepping scheme used in the simulations. Changing the time-stepping scheme, i.e. using different minimum and maximum system timesteps - which affects the energy conservation in the simulations - also leads to a different evolution of $\Omega_{\mathrm{p}}$ (Figs 7.12).

To summarize, a combination of different numerical effects has a significant influence on the evolution of $\Omega_{\mathrm{p}}$ and numerical simulations of small or even moderate $N$ do not allow a robust determination of the pattern speed.

\subsection{Summary}

In this work we present a systematic study of the influence of numerical effects on the evolution of the pattern speed of bars in fully self-consistent $N$-body/SPH simulations. We have applied different numerical schemes for computing the gravitational forces, such as a treecode or the GRAPE hardware. We found that the evolution of $\Omega_{\mathrm{p}}$ is very sensitive to both the intrinsic numerical noise of the model, as well as to the numerical accuracy of the method used. The superposition of these effects lead to an uncertainty of roughly 13 per cent in $\Omega_{p}$ for models with less than a few tens-of-thousands of particles and/or moderate force computations. We conclude that large $N$ and high force accuracy are required for making a robust determination of $\Omega_{\mathrm{p}}$.

\subsection{Acknowledgments}

We would like to thank Albert Bosma for interesting discussions, and J.C. Lambert for his computer assistance. IB acknowledges support from grants DFG Fr 325/48- 
2, /48-3 and Volkswagen Foundation I/76 520. He thanks the DFG for funding the grape facilities at the Sternwarte Göttingen. $\mathrm{CH}$ acknowledges support from grants HST-AR-09546.02-A, NSF-AST-0206251, GSU-FRG-2002, and DFG Fr 325/391, /39-2. EA would like to thank the IGRAP, the INSU/CNRS and the University of Aix-Marseille I for funds to develop the grape facilities used for part of the calculations in this paper.

\subsection{Bibliography}

Athanassoula E., 1996, in Buta R., Crocker D.A., Elmegreen B.G., eds, ASP Conf. Ser. Vol. 91, Barred Galaxies. Astron. Soc. Pac., San Francisco, p. 309

Athanassoula E., 2003, MNRAS, 341, 1179

Athanassoula E., Bosma A., Lambert J.-C., Makino J., 1998, MNRAS, 293, 369

Athanassoula E., Misiriotis A., 2002, MNRAS, 330, 35

Barnes J., Hut P., 1986, Nature, 324, 466

Barnes J., Hut P., 1989, ApJS, 70, 389

Berentzen I., Athanassoula E., Heller C.H., Fricke K.J., 2003, MNRAS, 341, 343

Canzian B., 1993, ApJ, 414, 487

Debattista V.P., 2003, MNRAS, 342, 1194

Debattista V.P., Sellwood J.A., 1998, ApJ, 493, L5

Debattista V.P., Sellwood J.A., 2000, ApJ, 543, 704

Dehnen W., 2000, ApJ, 536, L39

Elmegreen B.G., Elmegreen D.M., 1990, ApJ, 355, 52

Elmegreen D.M., Elmegreen B.G., Seiden P.E., 1989, ApJ, 343, 602

Heller C.H., 1991, Ph.D. thesis, Yale University

Heller C.H., Shlosman I., 1994, ApJ, 424, 84

Hernquist L., Weinberg M.D., 1992, ApJ, 400, 80

Hugoniot P.H., 1885, J. de l'Ecole Polytechnique, 57, 1

Kawai A., Fukushige T., Makino J., Taiji M., 2000, PASJ, 52, 659

Knapen J.H., Beckman J.E., Cepa J., van der Hulst T., Rand R., 1992, ApJ, 385, L37

Kuzmin G., 1956, Astron. Zh, 33, 27

Laine S., Shlosman I., Heller C.H., 1998, MNRAS, 297, 1052L

Lindblad P.A.B., Kristen H., 1996, A\&A, 313, 733

Lindblad P.A.B., Lindblad P.O., Athanassoula E., 1996, A\&A, 313, 65

Monaghan J.J., 1992, ARA\&A, 30, 543

Okumura S.K., Makino J., Ebisuzaki T., Fukushige T., Ito T., Sugimoto D., Hashimoto E., Tomida K., Miyakawa N., 1993, PASJ, 45, 329

Patsis P.A., Athanassoula E., 2000, A\&A, 358, 45

Plummer H.C., 1911, MNRAS, 71, 460

Rankine W.J.M., 1870, Phil. Trans. Roy. Soc. London, 160, 277

Sempere M.J., García-Burillo S., Combes F., Knapen J.H., 1995a, A\&A, 296, 45 
Sempere M.J., Combes F., Casoli F., 1995b , A\&A, 299, 371

Spitzer L., 1942, ApJ, 95, 329

Steimetz M., 1996, MNRAS, 278, 100

Sugimoto D., Chikada Y., Makino J., Ito T., Ebisuzaki T., Umemura M., 1990, Nat, 345,33

Toomre A., 1963, ApJ, 138, 385

Tremaine S., Weinberg M.D., 1984, ApJ, 282, L5

\subsection{Appendix: Shocks in a polytropic gas}

The continuity conditions for a shock at rest are obtained from the conservation of mass, momentum and energy given by:

$$
\begin{aligned}
& \rho_{1} v_{1}=\rho_{2} v_{2} \equiv j \\
& p_{1}+\rho_{1} v_{1}^{2}=p_{2}+\rho_{2} v_{2}^{2}
\end{aligned}
$$

and

$$
w_{1}+\frac{1}{2} v_{1}^{2}=w_{2}+\frac{1}{2} v_{2}^{2}
$$

where $\rho, p, w$ and $j$ are the density, the pressure, the enthalpy and the mass flux of the gas, and the indices $i=1,2$ denote the supersonic and the subsonic part of the gas. From these equations we get the equation of a shock adiabate (or HugoniotRankine adiabate; Rankine 1870; Hugoniot 1885), which is given by

$$
w_{1}-w_{2}+\frac{1}{2}\left(V_{1}+V_{2}\right)\left(p_{2}-p_{1}\right)=0,
$$

where $V$ is the specific volume of the gas, given by $V=1 / \rho$. For a polytropic gas, i.e. with a constant specific heat capacity $c_{\mathrm{p}}$, the enthalpy $w$ is given by:

$$
w=c_{\mathrm{p}} T=\frac{\gamma}{\gamma-1} p V=\frac{c_{\mathrm{s}}^{2}}{\gamma(\gamma-1)},
$$

where $c_{\mathrm{s}}$ is the sound speed, $\gamma$ the adiabatic index, and $T$ the temperature of the gas, respectively. From Eq. 7.11 we then get:

$$
\frac{V_{2}}{V_{1}}=\frac{(\gamma+1) p_{1}+(\gamma-1) p_{2}}{(\gamma-1) p_{1}+(\gamma+1) p_{2}}
$$

Using this relation we can now derive the ratios of density, pressure and temperature for the shock wave. With the definition of the Mach number Ma $=v / c_{\mathrm{S}}$ we obtain:

$$
\frac{\rho_{2}}{\rho_{1}}=\frac{v_{2}}{v_{1}}=\frac{(\gamma+1) \mathrm{Ma}_{1}^{2}}{(\gamma-1) \mathrm{Ma}_{1}^{2}+2},
$$




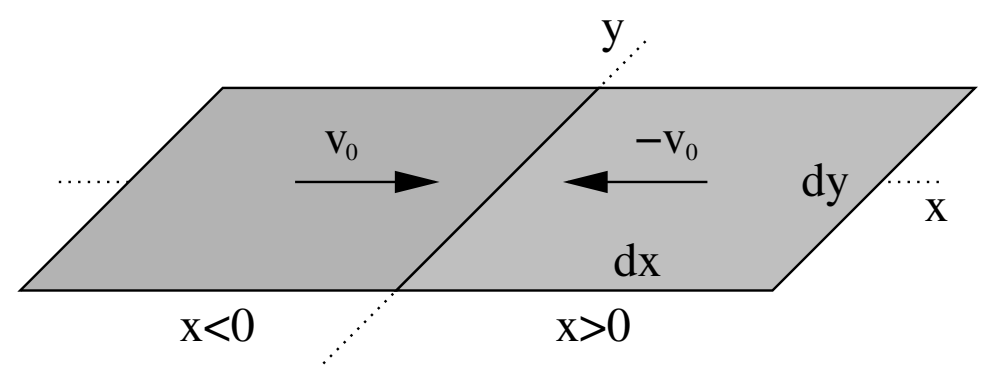

Figure 7.18: Systematic illustration of the initial set-up of the collision of two gaseous sheets.



Figure 7.19: Illustration of the initial set-up of the collision of two gaseous sheets.

$$
\frac{p_{2}}{p_{1}}=\frac{2 \gamma \mathrm{Ma}_{1}^{2}}{\gamma+1}-\frac{\gamma-1}{\gamma+1}
$$

and

$$
\frac{T_{2}}{T_{1}}=\frac{\left[2 \gamma \mathrm{Ma}_{1}^{2}-(\gamma-1)\right]\left[(\gamma-1) \mathrm{Ma}_{1}^{2}+2\right]}{(\gamma+1)^{2} \mathrm{Ma}_{1}^{2}}
$$

With these results we can now describe the collision between two isothermal sheets of gas, which collide with a relative velocity of $2 v_{0}=2 \mathrm{Ma}$ (see Fig. 7.18). This problem is equivalent to a gas with constant density and pressure, which is initially at rest, and bounded on the left by a plane piston. We further assume that the gas is compressed at an initial time by the piston moving into the gas with a constant velocity $u$, equal to the gas velocity. If $D$ is the propagation velocity of the shock through the stationary gas, then $v_{2}=-D$ is the velocity at which the undisturbed gas flows into the discontinuity. Likewise, $D-u$ is the propagation velocity of the discontinuity with respect to the gas moving behind it, and $v_{1}=-(D-u)$ is the velocity of the gas flowing out of the discontinuity. 
In this notation we finally get

$$
\begin{aligned}
& \frac{v_{2}}{v_{1}}=\frac{-D}{-(D-u)} \\
& \frac{\rho_{2}}{\rho_{1}}=\frac{(\gamma+1) \mathrm{Ma}_{1}^{2}}{(\gamma-1) \mathrm{Ma}_{1}^{2}+2} \\
& =\frac{(\gamma+1) D^{2}}{(\gamma-1) D^{2}+2 c_{\mathrm{s}, 1}^{2}},
\end{aligned}
$$

where we used $\mathrm{Ma}_{1}^{2}=v_{1}^{2} / c_{\mathrm{s}, 1}^{2}=D^{2} / c_{\mathrm{s}, 1}^{2}$. In Fig. 7.20 we show the evolution of the shock as a function of time. 

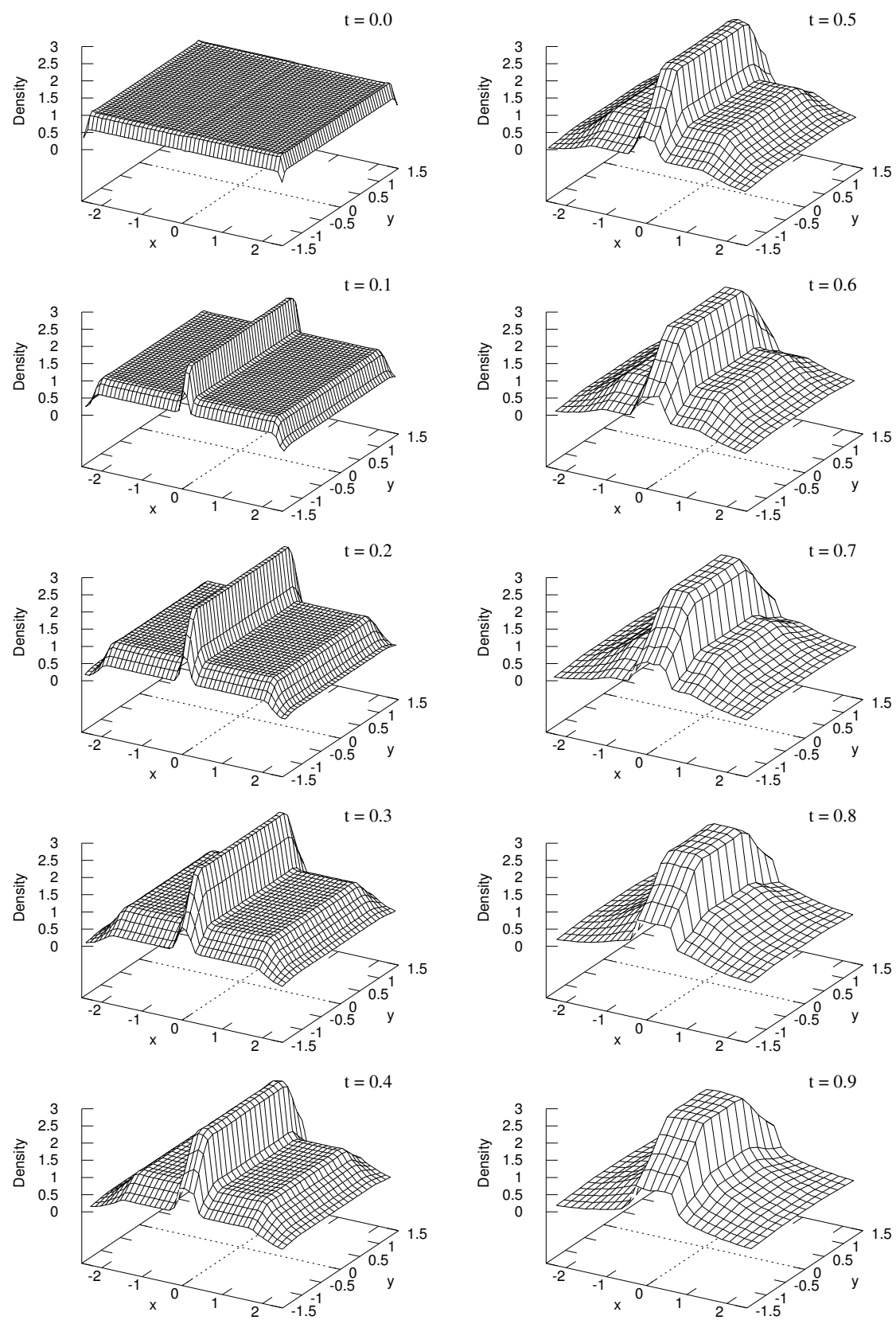

Figure 7.20: Evolution of the shock wave. 


\section{Summary and prospects}

The evolution of disc galaxies is driven by both internal and external processes, such as bars and interactions. Both these phenomena are now known to be common events in the life of disc galaxy. Thus interactions of barred galaxies should also be a common occurrence. However, numerical simulations of interacting disc galaxies have mainly concentrated on normal spiral galaxies only, i.e. without a central stellar bar.

The presence of gas has also been shown to be important to the dynamical evolution of barred galaxies. The inflow of gas due to the gravitational torques of bar and the resulting increase in the central gas concentration will weaken or destroy the bar. As the bar slows its structure and pattern speed has been shown to change. It is therefore expected that the inclusion of gas in models of barred galaxy interactions would be an important, if not critical, dynamical component.

In this work we have studied the effect of interactions between a gas-rich, initially barred galaxy and a companion galaxy by means of fully self-consistent numerical simulations. We modified an $N$-body/SPH code to handle multiple galaxy systems, along with the inclusion of additional routines to support both the GRAPE3 and GRAPE-5 hardware. Furthermore, several algorithms have been developed to set up the initial models and interaction configurations. Part of the simulations presented in this work have been performed on the Göttinger GRAPE-3 facilities, which is funded by the Deutsche Forschungsgemeinschaft (DFG) for this project. The hardware has been set up and fully maintained by the author.

\subsection{Vertical impacts of small companions}

In the first part of this work (Sec. 5) we have performed $N$-body/SPH simulations of an interaction between an initially barred galaxy and a less massive spherical companion. The initial conditions for the galaxies have been chosen such that the companion passes almost vertically through the disc of the host galaxy, leaving the host system following the impact. Both the impact time and the impact position have been varied with respect to the orientation of the bar and its evolutionary phase. The initial models and parameters in our simulations have been chosen to be similar to the ones used by Athanassoula, Puerari \& Bosma (1997) in order to provide a direct comparison with their purely stellar simulations. These purely collisionless simulations have shown that off-centred impacts of a sufficiently massive 
companion hitting the inner parts of a barred disc galaxy can displace the bar to one side, causing asymmetries and the formation of rings.

We find that the interactions produce characteristic features, such as expanding rings, tidal arms and off-centred bars, as observed in many interacting galaxies. In order to analyze the models accurately, we have developed several tools, e.g. for tracing the off-centred bars and the expanding rings automatically. We also developed graphical tools to study the 3D morphology of the galaxies. The features produced by the interaction remain in the disc for only a few dynamical times, in agreement with what is found in previous purely stellar simulations. The gaseous features in our models, however, tend to persist longer than their stellar counterparts, though after about $1 \mathrm{Gyr}$ both of them are dissolved.

The most striking feature produced in our central-impact models are the expanding rings. Owing to the passage of the companion through the disc both radial and vertical oscillations in the disc are excited. The induced radial oscillatory motion of the particles produces expanding density ring-waves (see, for example, Lynds \& Toomre 1976) centred on the impact position, while the vertical oscillations lead to an increase of the vertical velocity dispersion and a significant thickening of the disc. The same type of vertical motion can be seen in the numerical models by Lynds \& Toomre (1976) and also in Mihos \& Hernquist (1994b). While only two rings form in the stellar disc, we find several consecutive expanding ring features in the gas. The gas fragments that detach from the inner side of the first ring flow back to the central disc region and are sheared out by the differential rotation of the disc, forming several spokes between the two collisional rings (Hernquist \& Weil 1993).

In contrast to central impacts, the off-centred passages produce outward-expanding ring-like density waves not in the form of closed rings, but as long tidal spiral arms whose extent exceeds the initial disc cut-off radius. These tidal arms are present for a few dynamical times in our simulations, before they dissolve and the material in the arms is redistributed in the disc. We furthermore find that the bar gets off-centred in these simulations, which is in agreement with the results by Gerber \& Lamb (1994, 1996), Athanassoula (1996a) and Athanassoula et al. (1997). Asymmetries of this type are frequently found in disc galaxies, particularly in latetype galaxies (see, for example, de Vaucouleurs \& Freeman 1972; Odewahn 1996). While this kind of asymmetry could be produced by strong $m=1$ modes in the disc, we find that an off-centred impact of a companion galaxy should generally give rise to more significant shifts, depending on the impact parameters. The size of the displacement found in our models is in good agreement with the relative displacement parameter found by Feitzinger (1980) for Magellanic-type galaxies. The off-centring of the bar is also accompanied by asymmetries in the spiral structure. The morphology of the discs with off-centred bars in our simulations is dominated by one long spiral arm, which forms after the impact by an expanding density wave, in agreement with the results of Athanassoula (1996b) and with the morphology of several asymmetric barred galaxies as, e.g., NGC 4027. In all our models with offset passages we notice a strong displacement of the bar structure for approximately 
0.6 Gyr, independent of the evolutionary phase of the bar at impact time.

Central impacts and passages through the bar minor axis cause more damage to the bar than major axis impacts. In the central impact models the destruction of the bar is accompanied with a considerable thickening of the disc. For the minor axis impacts it is possible to destroy the stellar bar, while keeping the disc. This is in contrast to the pure $N$-body simulations, where an interaction of sufficient strength to destroy the bar also destroys the disc (Athanassoula 1996b), i.e. the ratio between the vertical and the radial scale-heights is greatly increased. The impact time itself, or correspondingly the dynamical phase of the disc, does not play a significant role in determining the final morphology of the disc. In most of our simulations, the stellar bar is destroyed after the impact of the companion. The dense nuclear disc and the surrounding gap in the gas distribution are the only morphological imprints that survive the interaction, even after the bar has dissolved. Such features in a non-barred galaxy may indicate the former existence of a bar.

We conclude that the fate of the bar is more sensitive to the impact location of the companion relative to the bar than to the evolutionary phase of the bar during impact. We finally argue that the interaction with a companion can drive the transition from a strongly-barred galaxy to a weakly-barred galaxy on shorter time-scales than those found in isolated models.

\subsection{The regeneration of bars}

Numerical simulations of isolated barred galaxies including a gaseous component have shown that the bar in the disc can be significantly weakened or dissolved during its evolution on relatively short time-scales. Thus the question has arisen if there are different epochs of bar formation and if stellar bars can be regenerated in order to explain the number of barred galaxies observed along the Hubble sequence (Sellwood \& Moore 1999; Friedli 1999; Athanassoula et al. 2000). Two scenarios have been suggested for the regeneration of stellar bars by Sellwood \& Moore (1999), i.e. by adding cooler material to the disc or by the tidal interaction with a companion galaxy. Numerical simulations have shown that bars can be triggered in initially non-barred galaxies by tidal interactions, and that those bars are slow rotators, while the spontaneously formed bars have higher pattern speeds. The presence of a former stellar bar, however, will changed the dynamics of the disc, for example increasing the velocity dispersion in the stellar disc and changing both the density and angular momentum distribution. Therefore, the regeneration of stellar bars is subject to different conditions than the formation of a bar in an bar-unstable isolated disc or in a tidal interaction. It has been unclear what kind of interactions are sufficient to regenerate the bar and what the properties of those bars would be.

In the second part (Sec. 6) of this work we therefore have used numerical simulations to investigate the regeneration of a stellar bar by tidal encounters. The host galaxy in our simulations again has been chosen to be initially bar unstable and 
forms a large-scale bar during its early evolution. Owing to the induced gas inflow the bar weakened significantly before the interaction with the companion takes place. We have used different masses and orbits for the companion in order to cover as wide a parameter range as possible for the interactions. Furthermore we have used two different host galaxies, one with and one without gas in order to elucidate the influence of the gas.

We find that tidal interactions, which are sufficiently strong to regenerate the bar in the purely stellar models, do not lead to a regeneration in the dissipative models. We argue that owing to the interaction, additional gas is driven towards the centre of the disc, which prevents the regeneration process. To analyze the bars we developed several tools to trace and classify individual orbits of the stellar particles.

The regenerated bars in our purely stellar simulations are long-living phenomena and by no means only transient, being in agreement with the results of Noguchi (1987) and Gerin et al. (1990). We argue that (regenerated) bars formed by interactions may indeed contribute to the number of barred spirals at high redshifts and in the local Universe (Sheth et al. 2003). Furthermore, the regenerated bars are generally slow-rotators and have lower pattern speeds than the bars in the corresponding isolated models, in agreement with what is found for tidally induced-bars in normal spiral galaxies (Miwa \& Noguchi 1998). We find a strong correlation between the strength and the pattern speed of the bar. This correlation is also in very good agreement with the correlation found for bars in isolated discs (Athanassoula 2003). This is one of the pieces of evidence indicating that the regenerated bars are qualitatively similar to those formed in isolated discs and thus cannot easily (if at all) be distinguished by comparing their dynamical properties.

Athanassoula (2003) argued that the evolution of bars in isolated disc galaxies is driven by the redistribution of angular momentum. These recent results argue strongly that the strength and the pattern speed of the bar are determined by the amount of angular momentum exchanged. In our models we find that the strength of the regenerated bars increases with the interaction strength. Owing to the tidal perturbation, angular momentum is removed from the disc. In fact, the whole disc within its initial cut-off radius loses angular momentum, in contrast to what is found for an isolated disc, where the corotation radius of the bar separates the disc into regions of angular momentum loss and gain (Athanassoula 2003). The amount of angular momentum removed from the disc shows a clear correlation with the interaction strength. We argue that the main effect of this angular momentum loss is a significant extension of the region where bar-supporting orbits exist, resulting in a lengthening of the bar. As a further, though somewhat less important effect, we find a thinning of both the bar-supporting orbits and the bar itself.

We finally conclude that the regeneration of stellar bars by galaxy interactions seems to be a reasonable mechanism, provided the external forcing is sufficiently strong. 


\subsection{Numerical accuracy of pattern speeds}

With the GRAPE-5 hardware becoming available, we performed a comparison of simulations of identical models using both the GRAPE-3 and the GRAPE-5. We find that although the morphological evolution of the disc was in qualitative agreement, the evolution of the bar's pattern speed showed differences of up to 13 per cent between the two machines. Since the pattern speed is one of the main parameters characterizing the dynamical properties of the bar and disc, the reliability of its measurement in numerical models is an important issue.

Thus we have presented in Sec. 6 the first systematic study of numerical effects on the accuracy of the pattern speed of isolated barred models. We show that the variations of $\Omega_{\mathrm{p}}$ found in our models result from a mix of different numerical effects, which are difficult to disentangle.

On the one hand there is the intrinsic numerical noise in the models, which can result in a different evolution of the bar. This noise owing to discreteness effects can likely be reduced with higher particle number. On the other hand, there are effects which are directly related to the numerical accuracy of the code and the hardware used. One aspect of this is the accuracy of the force calculation used in the simulations. The deviation of $\Omega_{\mathrm{p}}$ shows a clear correlation with the numerical accuracy when using a treecode and the different GRAPE machines. The results found are generally in agreement with the assumption of a gaussian-like distribution for the deviations in $\Omega_{\mathrm{p}}$. This is however based on only four different models and further simulations are required to improve the statistics.

Another important aspect is the time-stepping scheme used in the simulations. Changing the time-stepping scheme also leads to a different evolution of $\Omega_{\mathrm{p}}$.

To summarize, a combination of different numerical effects have a significant influence on the evolution of $\Omega_{\mathrm{p}}$ and numerical simulations of moderate $N$ or accuracy do not allow a robust determination of the pattern speed.

\subsection{Future research}

We have studied the effects of an interaction on the evolution of gas-rich barred galaxies, describing how interactions effect the evolution of the bar and the interstellar medium in the disc. In this work we have restricted ourselves to certain geometries of the interaction and to relatively fast passages, for which the two galaxies do not finally merge. However, numerical simulations have shown, that minor mergers also affect the evolution of disc galaxies in many ways, without actually destroying the disc. Purely stellar simulations of minor merger models with barred galaxies have shown that such an event can lead to the destruction of the bar and may result in the formation of a lens (Athanassoula 1996a; Athanassoula 1996b). Thus it would be interesting to study the effect of the accretion of either gas clouds or small companions on the evolution of gas-rich barred galaxy. 
Purely stellar simulations have shown a clear correlation between the pattern speed and the bar strength due to angular momentum transport within the disc and between the disc and the spheroidal components of the galaxy. These results, however, did not take the gaseous component into account, which would have a strong effect on the angular momentum transfer in the disc. It would be interesting to compare the properties of bars in gas-rich barred galaxies with the ones of the purely stellar models of Athanassoula (Athanassoula 2003).

The present work has emphasized the importance of the interstellar medium on the evolution of interacting barred galaxies. The gas, however, may be partially consumed by star-formation, which is likely to affect the evolution of the disc. The next step after studying the effects of the gaseous component alone would to include star-formation, with the accompanying gas consumption and energy feedback.

Star-formation may provide further interesting aspects in the evolution of collisions with barred galaxies. As we have shown in the simulations of vertical impacts, the final face-on morphology of the gaseous disc - in which the differences are more clear than in the stellar - the interacting and non-interacting models can hardly be separated. This might become possible from models including star formation, e.g., from the resulting colour/metallicity gradients. 


\section{Bibliography}

A

Aarseth S.J., Hénon M., Wielen R., 1974, A\&A, 37, 183

Arp H.C., 1966, Atlas of Peculiar Galaxies. California Institut of Technology, Pasadena.

Athanassoula E., 1992a, MNRAS, 259, 328

Athanassoula E., 1992b, MNRAS, 259, 345

Athanassoula E., 1994, in Shlosman I., Mass-Transfer Induced Activity in Galaxies. Cambridge Univ. Press, p.143

Athanassoula E., 1996a, in Buta R., Crocker D.A., Elmegreen B.G., eds, ASP Conf. Ser. Vol. 91, Barred Galaxies. Astron. Soc. Pac., San Francisco, p.309

Athanassoula E., 1996b, in Sandquist Aa., Lindblad P.O., eds, Lecture Notes in Physics Vol. 474, Barred Galaxies and Circumnuclear Activity. Springer Verlag, p. 59

Athanassoula E., 2000, review in the XIVth IAP meeting "Galaxy Dynamics, from the Early Universe to the Present"

Athanassoula E., 2002, ApJ, 569, L86

Athanassoula E., 2003, MNRAS, 341, 1179

Athanassoula E., Martinet L., 1980, A\&A, 87, L10

Athanassoula E., Misiriotis A., 2002, MNRAS, 330, 35

Athanassoula E., Sellwood J.A., 1986, MNRAS, 221, 195

Athanassoula E., Bienayme O., Martinet L., Pfenniger D., 1983, A\&A, 127, 349

Athanassoula E., Puerari I., Bosma A., 1997, MNRAS, 286, 284

Athanassoula E., Bosma A., Lambert J.-C., Makino J., 1998, MNRAS, 293, 369

Athanassoula E., Fady E., Lambert J.C., Bosma A., 2000, MNRAS, 314, 475

B

Barnes J.E., 1988, ApJ, 331, 699

Barnes J.E., 1992, ApJ, 393, 484

Barnes J.E., 1998, in Friedli D., Martinet L., Pfenniger D., eds, Galaxies: Interactions and Induced Star Formation. Springer, Heidelberg, p. 105

Barnes J.E., Hernquist L.E., 1991, ApJ, 370, 65

Barnes J.E., Hernquist L., 1992, ARA\&A, 30, 705

Barnes J.E., Hernquist L., 1996, ApJ, 471, 115

Barnes J.E., Hernquist L., 1998, ApJ, 495, 187

Barnes J.E., Hut P., 1986, Nat, 324, 446

Barnes J.E., Hut P., 1989, ApJS, 70, 389 
Benz W., 1990, in Buchler J.R., ed., The Numerical Modelling of Nonlinear Stellar Pulsations, Problems and Prospects. Kluwer, Dordrecht, Netherlands, p. 269

Berentzen I., Heller C.H., Shlosman I., Fricke K.J., 1998, MNRAS 300, 49

Berentzen I., Athanassoula E., Heller C.H., Fricke K.J., 2003, MNRAS, 341, 343

Binney J., Tremaine S., 1987, Galactic Dynamics. Princeton Univ. Press, Princeton Blitz L., Spergel D.N., 1991, ApJ, 379, 631

Bournaud F., Combes F., 2002, A\&A, 392, 83

Bureau M., Freeman K.C., 1997, Proc. Astron. Soc. Australia, 14, 146

Buta R. J., 1983, BAAS, Vol. 15, p. 659

Buta R. J., 1984, PhD thesis, Texas Univ., Austin.

Byrd G.G., Valtonen M.J., Valtaoja L., Sundelius B., 1986, A\&A, 166, 75

C

Canzian B., 1993, ApJ, 414, 487

Chamberlin T.C., 1901, ApJ, 14, 17

Chandrasekhar S., 1942, in Principles of Stellar Dynamics. Chicago Univ. Press, Chicago

Combes F., Gerin M., 1985, A\&A, 150, 327

Combes F., Debbasch D., Friedli D., Pfenniger D., 1990, A\&A, 233, 82

Contopoulos G., Grosbøl P., 1989, A\&ARv, 1, 261

Contopoulos G., Mertzanides C., 1977, A\&A, 61, 477

Contopoulos G., Papayannopoulos, 1980, A\&A, 92, 33

D

Dahari O., 1984, AJ, 89, 966

Debattista V.P., 2003, MNRAS, 342, 1194

Debattista V.P., Sellwood J.A., 1998, ApJ, 493, 5

Debattista V.P., Sellwood J.A., 2000, ApJ, 543, 70

Dehnen W., 2000, ApJ, 536, L39

Dehnen W., 2001, MNRAS, 324, 273

de Vaucouleurs G., 1963, ApJS, 8, 31

de Vaucouleurs G., 1964, in Kerr F.J. \& Rodgers A.W., eds., The Galaxy and the

Magellanic Clouds. IAU Symposium 20, Sydney: Australian Academy of Science, p. 195

de Vaucouleurs G., Freeman K.C., 1972, Vistas in Astronomy, 14, 163

E

Eddington A.S., 1916, MNRAS, 76, 572

Edmunds M.G., Roy J.-R., 1993, MNRAS, 261, L17

Elmegreen B.G., Elmegreen D.M., 1985, ApJ, 288, 438

Elmegreen B.G., Elmegreen D.M., 1990, ApJ, 355, 52

Elmegreen D.M., Elmegreen B.G., Seiden P.E., 1989, ApJ, 343, 602

Elmegreen D. M., Sundin M., Sundelius B., Elmegreen B., 1991, A\&A, 244, 52

Eskridge P.B. et al., 2000, AJ, 119, 536

Evrard A.E., 1988, MNRAS, 235, 911 
$\mathbf{F}$

Feitzinger J.V., 1980, Space Sci. Review, 27, 35

Fridman A.M., Polyachenko V.L., 1984, Physics of Gravitating Systems. New York: Springer

Friedli D., 1999, in Beckman J.E., Mahoney T.J., eds, ASP Conf. Ser. Vol. 187, The Evolution of Galaxies on Cosmological Timescales. Astron. Soc. Pac., San Francisco, p. 88

Friedli D., Benz W., 1993, A\&A, 268, 65

Friedli D., Pfenniger D., 1991, A\&A, 252, 75

G

Gerber R.A., Lamb S.A., 1994, ApJ, 431, 604

Gerber R.A., Lamb S.A., 1996, MNRAS, 278, 345

Gerhard O.E., 1996, in Blitz L., Teuben P., eds, Proc. IAU Symp. 169, Unresolved Problems of the Milky Way. Kluwer, Dordrecht, p. 79

Gerin M., Combes F., Athanassoula E., 1990, A\&A, 230, 37

Gingold R.A., Monaghan J.J., 1977, MNRAS, 181, 375

$\mathbf{H}$

Hasan H., Norman C.A., 1990, ApJ, 361, 69

Hasan H., Pfenniger D., Norman C.A., 1993, ApJ, 409, 91

Heckman T.M., 1990, in Sulentic J.W., Keel W.C., Telesco C.M., eds, Paired and Interacting Galaxies. IAU Colloq. 124, p.359

Heckman T.M., 1994, in Shlosman I., eds, Mass-Transfer Induced Activity in Galaxies. Cambridge, Cambridge Univ. Press, p.234

Heller C.H., 1991, Ph.D. thesis, Yale University

Heller C.H., Shlosman, I., 1994, ApJ, 424, 84

Heller C.H., Shlosman, I., 1996, ApJ, 471, 143

Hernquist L., 1992, ApJ, 400, 460

Hernquist L., 1993, ApJ, 409, 548

Hernquist L., Katz N., 1989, ApJS, 70, 419

Hernquist L., Mihos J.C., 1995, ApJ, 448, 41

Hernquist L., Weil M.L., 1993, MNRAS, 261, 804

Hernquist L., Weinberg M.D., 1992, ApJ, 400, 80

Higdon J.L., 1995, ApJ, 455, 524

Hockney R.W., Eastwood J.W., 1988, in: Computer Simulation using Particles, New York: Adam Hilger

Hohl F., 1971, ApJ, 168, 343

Holmberg E., 1941, ApJ, 94, 385

Holmberg E., 1958, Lund. Medd. Astron. Obs. Ser. II, 136, 1

Horellou C., Combes F., 2001, Ap\&SS, 276, 1141

Hubble P. E., 1926, ApJ, 64, 321

Hugoniot P.H., 1885, J. de l'Ecole Polytechnique, 57, 1

Hummel E., 1981, A\&A, 96, 111 
Hunter C., Toomre A.T., 1969, ApJ, 155, 747

K

Kawai A., Fukushige T., Makino J., Taiji M., 2000, PASJ, 52, 659

Keel W.C., Kennicutt R.C. Jr., Hummel E., van der Hulst J.M., 1985, AJ, 90, 708

Kennitcutt R.C. Jr., 1998, in Friedli D., Martinet L., Pfenniger D., eds, Galaxies: Interactions and Induced Star Formation. Springer, Heidelberg, p. 1

Kennicutt R.C. Jr., Keel W.C., 1984, ApJ, 279, L5

Knapen J.H., Beckman J.E., Cepa J., van der Hulst T., Rand R., 1992, ApJ, 385, L37

Korchagin V., Vorobyov E.I., Mayya Y.D., 1999, ApJ, 522, 767

Kormendy J., 1982, ApJ, 257, 75

Kuijken K., 1996, in Buta R., Crocker D.A., Elmegree B.G., eds, ASP Conf. Ser. Vol. 91, Barred Galaxies. Astron. Soc. Pac., San Francisco, p. 504

Kuzmin G., 1956, Astron. Zh, 33, 27

$\mathbf{L}$

Laine S., Heller C.H., 1999, MNRAS, 308, L557

Laine S., Shlosman I., Heller C.H., 1998, MNRAS, 297, 1052L

Larson R.B., Tinsley B.M., 1978, ApJ, 219, L46

Lindblad B., 1927, MNRAS, 87, 553

Lindblad P.A.B., Kristen H., 1996, A\&A, 313, 733

Lindblad P.A.B., Lindblad P.O., Athanassoula E., 1996, A\&A, 313, 65

Little B., Carlberg R.G., 1991, MNRAS, 250, 161

Little B., Carlberg R.G., 1991, MNRAS, 251, L227

Lucy L.B., 1977, AJ, 82, 1013L

Lynden-Bell D., 1979, MNRAS, 136, 101L

Lynden-Bell D., Kalnajs A.J., 1972, MNRAS, 157, 1L

Lynds R., Toomre A., 1976, ApJ, 209, 328

M

Marcum P.M., Appleton P.N., Higdon J.L., 1992, ApJ, 399, 57

Martin P., 1995, AJ, 109, 2428

Martin P., Friedli D., 1999, A\&A, 346, 769

Martin P., Roy J.-R., 1994, ApJ, 424, 599

Merrit D., 1996, AJ, 111, 2462

Mihos J.C., Hernquist L., 1994a, ApJ, 425, L13

Mihos J.C., Hernquist L., 1994b, ApJ, 437, 611

Miller R.H., Prendergast K.H., Quirk W.J., 1970, ApJ, 161, 903

Mirabel I.F., Lutz D., Maza J., 1991, A\&A, 243, 367

Mirabel I.F., Dottori H., Lutz D., 1992, A\&A, 256, L19

Miwa T., Noguchi M., 1998, ApJ, 499, 149

Monaghan J.J., 1992, ARA\&A, 30, 543

Monaghan J.J., Gingold R.A., 1983, J. Comput. Phys., 52, 374

Monaghan J.J., Lattanzio J.C., 1985, A\&A, 149, 135 


\section{$\mathbf{N}$}

Noguchi M., 1987, MNRAS, 228, 635

Noguchi M., 1988, A\&A, 203, 259

Noguchi M., 1996, ApJ, 469, 605

Norman C.A., Sellwood J.A., Hasan H., 1996, ApJ, 462, 114

$\mathbf{O}$

Odewahn S.C., 1996, in Buta R., Crocker D.A., Elmegreen B.G., eds, ASP Conf. Ser. Vol 91, Barred Galaxies. Astron. Soc. Pac., San Francisco, p. 30

Okumura S.K., Makino J., Ebisuzaki T., Fukushige T., Ito T., Sugimoto D., Hashimoto E., Tomida K., Miyakawa N., 1993, PASJ, 45, 329

Ostriker J.P., Peebles P.J.E., 1973, ApJ, 186, 467

$\mathbf{P}$

Patsis P.A., Athanassoula E., 2000, A\&A, 358, 45

Patsis P.A., Skokos Ch., Athanassoula E., 2002, MNRAS, 337, 578

Patsis P.A., Skokos Ch., Athanassoula E., 2003, MNRAS, 342, 69

Pèrez-Ramírez D., Knapen J.H., Peletier R.F., Laine S., Doyon R., Nadeau D., 2000, MNRAS, 317, 234

Pfenniger D., 1984, A\&A, 134, 373

Pfenniger D., Friedli D., 1991, A\&A, 252, 75

Pfleiderer J., 1963, Zs. f. Ap., 58, 12

Pfleiderer J., Siedentopf H., 1961, Zs. f. Ap., 51, 201

Plummer H.C., 1911, MNRAS, 71, 460

Poincaré H., 1892, Les Methods Nouvelles de la Mechanique Celeste, Paris: Gauthier-Villars

Pompea S.M., Rieke G.H., 1990, ApJ, 356, 416

Prendergast K.H., 1983, in Athanassoula E., ed., Internal kinematics and dynamics of galaxies. Reidel, Dordrecht, p.215

Press W.H., Teukolsky S.A., Vetterling W.T., Flannery B.P., 1986, Numerical Recipes. Cambridge Univ. Press, Cambridge

\section{Q}

Quinn P.J., Goodman J., 1986, ApJ, 309, 472

Quinn P.J., Hernquist L., Fullagar D.P., 1993, ApJ, 403, 74

$\mathbf{R}$

Raha N., Sellwood J.A., James R.A., Kahn F.D., 1991, Nat., 352, 411

Rankine W.J.M., 1870, Phil. Trans. Roy. Soc. London, 160, 277

Rix H.-W., 1993, PASP, 105, 999

Rix H.-W., Rieke M.J., 1993, ApJ, 418, 123

S

Sakamoto K., Okumura S.K., Ishizuki S., Scoville N.Z., 1999, ApJ, 525, 691

Salo H., 1991, A\&A, 243, 118

Sanders D.B., 1992, in Filippenko A.V., ed, Relationship between active galactic nuclei and starburst galaxies, ASP Conf. Series, Vol. 31, San Francisco, p. 303 
Sanders R.H., Teuben P.J., Van Albada G.D., 1983, in Athanassoula E., ed., Internal kinematics and dynamics of galaxies. Reidel, Dordrecht, p.221

Schwarz M.P., 1979, PhD thesis, Austr. Nat. Univ.

Schwarz M.P., 1981, ApJ, 247, 77

Schwarz M.P., 1984a, Proc. Astron. Soc. Australia, 5, 464

Schwarz M.P., 1984b, A\&A, 133, 222

Schwarz M.P., 1984c, MNRAS, 209, 93

Schwarzkopf U., Dettmar R.-J., 2000, A\&A, 361, 451

Schweizer F., 1978, in Berkhuisen E.M., Wiebilinski R., eds, Structure and Properties of Nearby Galaxies. Dordrecht D. Reidel, p.279

Schweizer F., 1998, in Friedli D., Martinet L., Pfenniger D., eds, Galaxies: Interactions and Induced Star Formation. Springer, Heidelberg, p. 275

Seigar M.S., James P.A., 1998, MNRAS, 299, 672

Sellwood J. A., 1980, A\&A, 89, 296

Sellwood J. A., 1981, A\&A, 99, 362

Sellwood J. A., 1987, ARA\&A, 25, 151

Sellwood J. A., Moore E.M., 1999, ApJ, 510, 125

Sellwood J.A., Sparke L.S., 1988, MNRAS, 231, 25

Sellwood J.A., Wilkinson A., 1993, Rep. Prog. Phys., 56, 173

Sempere M.J., García-Burillo S., Combes F., Knapen J.H., 1995a, A\&A, 296, 45

Sempere M.J., Combes F., Casoli F., 1995b , A\&A, 299, 371

Sheth K., Regan M.W., Scoville N., Sparke L., 2003, ApJL, in press

Shlosman I., Noguchi M., 1993, ApJ, 414, 474

Shlosman I., Begelman M.C., Frank J., 1990, Nat, 345, 679

Shlosman I., Frank J., Begelman M.C., 1989, Nat, 338, 45

Skokos Ch., Patsis P.A., Athanassoula E., 2002a, MNRAS, 333, 847

Skokos Ch., Patsis P.A., Athanassoula E., 2002b, MNRAS, 333, 861

Spitzer L., 1942, ApJ, 95, 329

Steinmetz M., 1996, MNRAS, 278, 1005

Struck C., 1999, preprint, astro-ph/9908269

Sugimoto D., Chikada Y., Makino J., Ito T., Ebisuzaki T., Umemura M., 1990, Nat, 345,33

Sundin M., Sundelius B., 1991, A\&A, 245, L5

Sundin M., Donner K.J., Sundelius B., 1993, A\&A, 280, 105

Sygnet J.F., Tagger M., Athanassoula E., Pellat R., 1988, MNRAS, 232, 733 $\mathbf{T}$

Tagger M., Sygnet J.F., Athanassoula E., Pellat R., 1987, ApJ, 318, 43L

Tremaine S., Weinberg M.D., 1984a, ApJ, 282, L5

Tremaine S., Weinberg M.D., 1984b, MNRAS, 209, 729

Toth G., Ostriker J.P., 1992, ApJ, 389, 5

Toomre A., 1963, ApJ, 138, 385

Toomre A., 1964, ApJ, 139, 1217 
Toomre A., 1977, in Tinsley B.M., Larson R.B., eds, Evolution of Galaxies and Stellar Populations. Yale Univ. Observ., p.401

Toomre A., 1978, in Longair M.S., Einasto J., eds, The large scale structure of the universe. Dordrecht, D. Reidel Publishing Co., p. 109

Toomre A., 1981, in Fall S.M., Lynden-Bell D., eds, Structure and Evolution of Normal Galaxies. Cambridge Univ. Press, Cambridge, p. 111

Toomre A., Toomre J., 1972, ApJ, 139, 179, 623 V

van der Kruit P.C., Freeman K.C., 1984, ApJ, 278, 81

van der Kruit P.C., Searle L., 1981, A\&A, 95, 116

Villa J., Athanassoula E., Bosma A., 2002, in Combes F., Barret D., Contini T., eds, Semaine de l'Astrophysique Francaise, EdP-Sciences, Conf. Ser., p. 141

Vorontsov-Velyaminov B.A., 1959, Atlas and Catalogue of Interacting Galaxies. Part I. Moscow Univ., Moscow

Vorontsov-Velyaminov B.A., 1977, A\&A Suppl. Ser., Atlas of Interacting Galaxies Part II, 28, 1

Vorontsov-Velyaminov B.A., Krasnogorskaya A., 1961, Morphological Catalogue of Galaxies. Moscow Univ., Moscow W

Weilbacher P., Duc P.-A., Fritze-v. Alvensleben U., Martin P., Fricke K.J., 2000, A\&A, 358, 819

Weilbacher P., Duc P.-A., Fritze-v. Alvensleben U., 2003, A\&A, 397, 545

Weinberg M., 1985, MNRAS, 213, 451

Weinberg M., 1991, ApJ, 373, 391

Weinberg M., Tremaine S., 1983, ApJ, 271, 586

Whitehurst R., 1988, MNRAS, 233, 529

White S.D.M., 1982, in Martinet L., Mayor M., eds, Morphology and Dynamics of Galaxies. Sauverny: Geneva Observatory, p. 291 $\mathbf{Z}$

Zaritsky D., Smith R., Frenk C., White S.D.M., 1993, ApJ, 405, 464

Zaritsky D., Smith R., Frenk C., White S.D.M., 1997, ApJ, 478, 39

Zwicky F., 1961, Catalogue of Galaxies and Clusters of Galaxies. California Institute of Technology, Pasadena 


\section{Appendices}





\section{A. Astronomical units and constants}

Constants relevant for this work:

\begin{tabular}{lll}
\hline Gravitational constant & $\mathrm{G}$ & $6.672(4) \times 10^{-8} \mathrm{~cm}^{3} \mathrm{~g}^{-1} \mathrm{sec}^{-2}$ \\
Mass of hydrogen & $m_{H}$ & $1.6733 \times 10^{-24} \mathrm{~g}$ \\
Boltzmann's constant & $k_{B}$ & $1.38066(4) \times 10^{-16} \mathrm{erg} \mathrm{K}^{-1}$ \\
Parsec & $\mathrm{pc}$ & $3.08567802(2) \times 10^{18} \mathrm{~cm}$ \\
Solar mass & $M \odot$ & $1.989(2) \times 10^{33} \mathrm{~g}$ \\
Hubble constant & $H_{0}$ & $100 h \mathrm{~km} \mathrm{~s}^{-1} \mathrm{Mpc}^{-1}$ where $0.5<h<1$ \\
Hubble time & $H_{0}^{-1}$ & $9.78 h^{-1} \times 10^{9} \mathrm{yr}$ \\
\hline
\end{tabular}

Table A.1: Units and constants

Reference: Binney \& Tremaine (1987) 
B. Documentation for the Göttinger $N$-body/SPH code

\section{B.1 Input files}

FTM.NML Simulation parameters namelist.

FTM.MSG Message text. Copied to output file FTM.TXT (optional).

FTM.MDL Model data.

\section{B.2 Output files}

FTM.TXT Informational and diagnostic text.

FTM.DMP Model diagnostic data dump.

FTM.RST Model restart file. Same as FTM.MDL, but with additional data.

Needed for restart appended to end of file. May be renamed to

FTM.MDL for restart.

FTM.ACC Accretion diagnostics.

FTM.SFM Star formation diagnostics.

\section{B.3 Input parameters (FTM.NML)}

\begin{tabular}{|c|c|c|}
\hline MODEL & ascii & $\begin{array}{l}\text { Model file specification. All input, except FTM.NML, } \\
\text { and all output files use this file name. MODEL = 'ftm' } \\
\text { in this document. }\end{array}$ \\
\hline SELFGRAV & logical & Gas self-gravity switch. \\
\hline KERNEL3D & logical & $\begin{array}{ll}\text { SPH kernel dimension. } \\
\begin{array}{ll}\text {.FALSE. } & \text { 2D } \\
\text {.TRUE. } & \text { 3D }\end{array}\end{array}$ \\
\hline QUADPOLE & logical & Gravitational quadrupole-moment switch. \\
\hline DYNSOFT & logical & $\begin{array}{ll}\text { SPH dynamic gravitational softening. } \\
\text {.FALSE. } & \text { EPS }=\text { SOFT_GRV } \\
\text {.TRUE. } & \text { EPS }=\text { SPH smoothing length }\end{array}$ \\
\hline SWITCH & logical & Artificial viscosity "switch". \\
\hline $\mathrm{XSPH}$ & logical & SPH marching velocities switch. \\
\hline EXTPOT & logical & $\begin{array}{l}\text { External gravitational potential switch. } \\
\text { Executes EXTINIT on startup and EXTFORCE }\end{array}$ \\
\hline
\end{tabular}




\begin{tabular}{|c|c|c|}
\hline \multirow{3}{*}{ ACCRETE } & \multirow{3}{*}{ logical } & during force evaluation. See EXTFORCE.F. \\
\hline & & Central accreting object switch. \\
\hline & & $\begin{array}{l}\text { If } R E S T A R T=0 \text {, initializes stellar particle } \\
\text { as central accreting object. }\end{array}$ \\
\hline STARFORM & logical & SPH star formation switch. \\
\hline ASSLOSS & logical & Stellar mass loss switch. \\
\hline \multirow[t]{2}{*}{ DAMP ING } & logical & Damping term switch. \\
\hline & & Used to produce equilibrium gas models. \\
\hline \multirow[t]{6}{*}{ STEPPING } & integer & $\begin{array}{l}\text { Time stepping method. } \\
0 \text { - Single time-sten }\end{array}$ \\
\hline & & $\begin{array}{l}0 \text { - Single time-step } \\
\text { STEP MIN = time-step size }\end{array}$ \\
\hline & & STEP_MAX = log output step size \\
\hline & & 1 - Hierarchical time bins \\
\hline & & STEP_MIN $=$ minimum step size for stars \\
\hline & & STEP_MAX $=$ maximum or system step size \\
\hline \multirow[t]{5}{*}{ RESTART } & integer & Simulation restart indicator. \\
\hline & & 0 - No restart; time $=0.0$ \\
\hline & & 1 - Restart; FTM.MDL contains restart data \\
\hline & & 2 - No restart; time $=0.0$, shift to c-of-m \\
\hline & & 3 - No restart; time $=0.0$, smooth fields \\
\hline \multirow[t]{4}{*}{ EQSTATE } & integer & Equation of state indicator. \\
\hline & & 1 - Perfect gas; $\mathrm{p}=($ GAMMA- 1$) \cdot \rho \cdot \mathrm{u}$ \\
\hline & & 2 - Polytropic; $\boldsymbol{p}=$ POLY_CON $\cdot \rho^{* *}$ GAMMA \\
\hline & & 3 - Isothermal; $\mathrm{p}=\mathrm{POLY} \_\mathrm{CON} * * 2 * \rho$ \\
\hline \multirow[t]{3}{*}{ EQVISC } & integer & Artificial viscosity indicator. \\
\hline & & $1-\boldsymbol{v} \cdot \boldsymbol{r}$ type \\
\hline & & $2-\nabla \cdot v$ type \\
\hline \multirow[t]{5}{*}{ DUMP INDX } & integer & Dump file format indicator. \\
\hline & & 1 -class, state, mass, $\mathrm{x}, \mathrm{v}$ \\
\hline & & $2-1+$ eps, phi, f \\
\hline & & $3-1+2+u, d, \operatorname{divv}, \operatorname{udot} 1, \operatorname{udot} 2, \mathrm{~h}$ \\
\hline & & See Data Base section for explanation of variables. \\
\hline \multirow[t]{6}{*}{ DATAFRMT } & integer & Floating point format indicator. \\
\hline & & 1 - IEEE \\
\hline & & $2-\mathrm{IBM}$ \\
\hline & & Translates FTM.MDL file into native format, writes \\
\hline & & ftm.rst file in native format; always writes \\
\hline & & ftm. dmp file in IEEE format. \\
\hline \multirow[t]{3}{*}{ TIME_TXT } & real & Textual information output time-step. \\
\hline & & Energy diagnostics output to stdout and FTM.TXT. \\
\hline & & Should be divisible by STEP_MAX. \\
\hline \multirow[t]{2}{*}{ TIME_DMP 1} & real & Stellar dump file output time-step. \\
\hline & & Full or partial dump selected by DUMP INDX. \\
\hline
\end{tabular}




\begin{tabular}{|c|c|c|}
\hline \multirow{4}{*}{ TIME_DMP 2} & \multirow{3}{*}{ real } & Should be divisible by STEP_MAX. \\
\hline & & Gas dump file output time-step. \\
\hline & & Full or partial dump selected by DUMP INDX. \\
\hline & & Should be divisible by STEP_MAX. \\
\hline \multirow[t]{2}{*}{ TIME_RST } & real & Restart file output time-step. \\
\hline & & Should be divisible by STEP_MAX. \\
\hline \multirow[t]{2}{*}{ TIME_END } & real & End time of simulation. \\
\hline & & Should be divisible by STEP_MAX. \\
\hline STEP_MAX & real & Maximum system time-step. \\
\hline STEP_MIN & real & Minimum time-step for collisionless particles. \\
\hline \multirow[t]{2}{*}{ ZONE_MX1 } & real & Outer removal zone one. \\
\hline & & $\begin{array}{l}\text { Particles greater than this distance from c-of-m of } \\
\text { system and having positive energies are removed } \\
\text { from the simulation. }\end{array}$ \\
\hline \multirow[t]{3}{*}{ ZONE_MX2 } & real & Outer removal zone two. \\
\hline & & $\begin{array}{l}\text { Particles greater than this distance from c-of-m of } \\
\text { system are removed from simulation. }\end{array}$ \\
\hline & & Usually ZONE_MX1 < ZONE_MX2. \\
\hline ZONE_MIN & real & Accretion radius of central object. \\
\hline TOL_GRAV & real & $\begin{array}{l}\text { Gravitational tolerance in tree algorithm (theta). } \\
\text { Usually TOL_GRAV }<=0.8 \text {. }\end{array}$ \\
\hline TOL_BODY & real & $\begin{array}{l}\text { Time-step tolerance for stellar particles. } \\
\text { Usually TOL_BODY }<0.05 \text {. }\end{array}$ \\
\hline TOL_SPH & real & $\begin{array}{l}\text { Time-step tolerance for SPH particles. } \\
\text { Usually TOL_SPH }<=0.3 \text {. }\end{array}$ \\
\hline \multirow[t]{2}{*}{ RAD_INIT } & real & Initial SPH smoothing length. \\
\hline & & $\begin{array}{l}\text { Assigned to particles at initialization if RAD_INIT } \\
\text { not equal to zero, else smoothing lengths from } \\
\text { model file (FTM.MDL) used. }\end{array}$ \\
\hline \multirow[t]{2}{*}{ RAD_INCR } & real & SPH smoothing length increment. \\
\hline & & $\begin{array}{l}\text { Used in iterative neighborhood routine if number } \\
\text { of neighbors equals zero. Usually set equal } \\
\text { to about } 10 \% \text { of system size. }\end{array}$ \\
\hline SOFT_GRV & real & $\begin{array}{l}\text { Gravitational softening length. } \\
\text { eps = SOFT_GRV if non-zero. Not used by SPH par- } \\
\text { ticles, if DYNSOFT }=\text {. TRUE. }(\operatorname{eps}(i)=\mathrm{h}(i)) .\end{array}$ \\
\hline SOFT_MIN & real & $\begin{array}{l}\text { Minimum gravitational softening length. } \\
\text { Used only if DYNSOFT }=. \text { TRUE. }\end{array}$ \\
\hline GAMMA & real & Ratio of specific heats. \\
\hline POLY_CON & real & Polytropic constant. \\
\hline & & $\begin{array}{l}\text { Used by equation of state. Equals sound speed if } \\
\text { isothermal. See EQSTATE above for more details. }\end{array}$ \\
\hline $\mathrm{XSPH} \mathrm{CON}$ & real & XSPH constant. $0<\mathrm{XSPH}$ _CON $<1$. \\
\hline
\end{tabular}




\begin{tabular}{|c|c|c|}
\hline SFM_DMAX & real & $\begin{array}{l}\text { Star formation maximum gas density. } \\
\text { Star formation is triggered when rho }>\text { SFM_DMAX } \\
\text { and } \nabla \cdot \boldsymbol{v}<0 \text {. }\end{array}$ \\
\hline SFM_EFCY & real & $\begin{array}{l}\text { Star formation efficiency factor. } \\
\text { Typically } 0.02<\text { SFM_EFCY }<0.5 \text {. }\end{array}$ \\
\hline SFM_RTRN & real & $\begin{array}{l}\text { Star formation mass return factor. } \\
\text { Typically SFM_RTRN }=0.45 \text {. }\end{array}$ \\
\hline SFM_SNII & real & $\begin{array}{l}\text { Number of SNII per unit mass. } \\
\text { Typically SFM_SNII }=0.002 \text { per solar mass. }\end{array}$ \\
\hline SFM_ENGY & real & $\begin{array}{l}\text { Energy per SNII. } \\
\text { Typically SFM_ENGY }=10^{51} \text { ergs. }\end{array}$ \\
\hline SFM_THRM & real & Star formation thermalization factor. \\
\hline SFM_WIND & real & Star formation wind factor. \\
\hline SFM_ZYLD & real & Star formation metal yield per unit mass. \\
\hline SFM_GMIN & real & $\begin{array}{l}\text { Minimum SPH particle mass. } \\
\text { Typically 5\% of SPH initial particle mass. }\end{array}$ \\
\hline MLS_GMAS & real & Mass loss SPH particle mass. \\
\hline MLS_NMIN & real & Mass loss minimum number of particles. \\
\hline VISC_P1 & real & $\begin{array}{l}\text { Artificial viscosity parameter one. } \\
\text { Typically VISC_P1 }=0.5 \text { for EQVISC }=1 \text { or } 2 .\end{array}$ \\
\hline VISC_P2 & real & $\begin{array}{l}\text { Artificial viscosity parameter two. } \\
\text { Typically VISC_P } 2=1.0 \text { for } \mathrm{EQVISC}=1 . \\
\text { Typically VISC_P } 2=1.5 \text { for } \mathrm{EQVISC}=2 .\end{array}$ \\
\hline DAMP_CON & real & $\begin{array}{l}\text { Damping factor. } \\
\text { Used to obtain equilibrium gaseous models. } \\
\text { Typically DAMP_CON }=0.3 \text {. }\end{array}$ \\
\hline
\end{tabular}

\section{B.4 Model and diagnostic data}

$\begin{array}{ll}\text { Class } \quad \text { integer } * 4 & \text { Index assigned to each particle. } \\ & \text { Used to both uniquely identify and classify } \\ & \text { individual particles. } \\ & \text { For compatibility with GALPLOT graphics: } \\ & \text { class }<-100,000 \text { Stellar halo particle } \\ & -100,000<=\text { class }<0 \text { Stellar disk particle } \\ & \text { class }=0 \text { Central BH } \\ & 0<\text { class SPH particle } \\ \text { state } \quad \text { integer } * 2 & \text { Bit map indicating state of particle. } \\ & \text { Currently only used to indicate SPH particle } \\ & \text { undergoing starformation: } \\ & 0-\text { No starformation } \\ & 1-\text { Starformation }\end{array}$




\begin{tabular}{|c|c|c|}
\hline \multirow{5}{*}{$\begin{array}{l}\text { mass } \\
\text { eps }\end{array}$} & real*8 & Mass of particle. \\
\hline & real*8 & Gravitational softening length of particle. \\
\hline & & Read in from FTM.MDL file. \\
\hline & & Set equal to SOFT_GRV, if non-zero. \\
\hline & & $\begin{array}{l}\text { For SPH particles, set equal to smoothing length } \\
\text { if DYNSOFT equals . TRUE.. }\end{array}$ \\
\hline$x$ & real*8 & Position vector of particle. \\
\hline $\mathrm{V}$ & real*8 & Velocity vector of particle. \\
\hline phi & real*8 & Gravitational potential of particle. \\
\hline f & real $* 8$ & Acceleration vector of particle. \\
\hline $\mathrm{u}$ & real $* 8$ & SPH Internal energy per unit mass. \\
\hline $\mathrm{d}$ & real*8 & SPH gas density. \\
\hline divv & real $* 8^{*}$ & SPH divergence of velocity field. \\
\hline udot 1 & real*8 & SPH du/dt adiabatic component. \\
\hline $\operatorname{dot} 2$ & real*8 & SPH du/dt viscous component. \\
\hline & real*8 & SPH smoothing length. \\
\hline
\end{tabular}

\section{B.5 Compile time parameters (PARAM.F)}

MXBODY1 Maximum number of collisionless (stellar) particles.

MXBODY2 Maximum number of collisional (gaseous) particles.

NEIOPT Optimum number of SPH neighbors.

NEITOL Neighbor tolerance. The actual number of neighbors, NEI,

is allowed to vary from NEIOPT-NEITOL to NEIOPT+NEITOL.

\section{B.6 Model data input file format (FTM.MDL)}

The following is an example FORTRAN code section illustrating the format of a model input file:






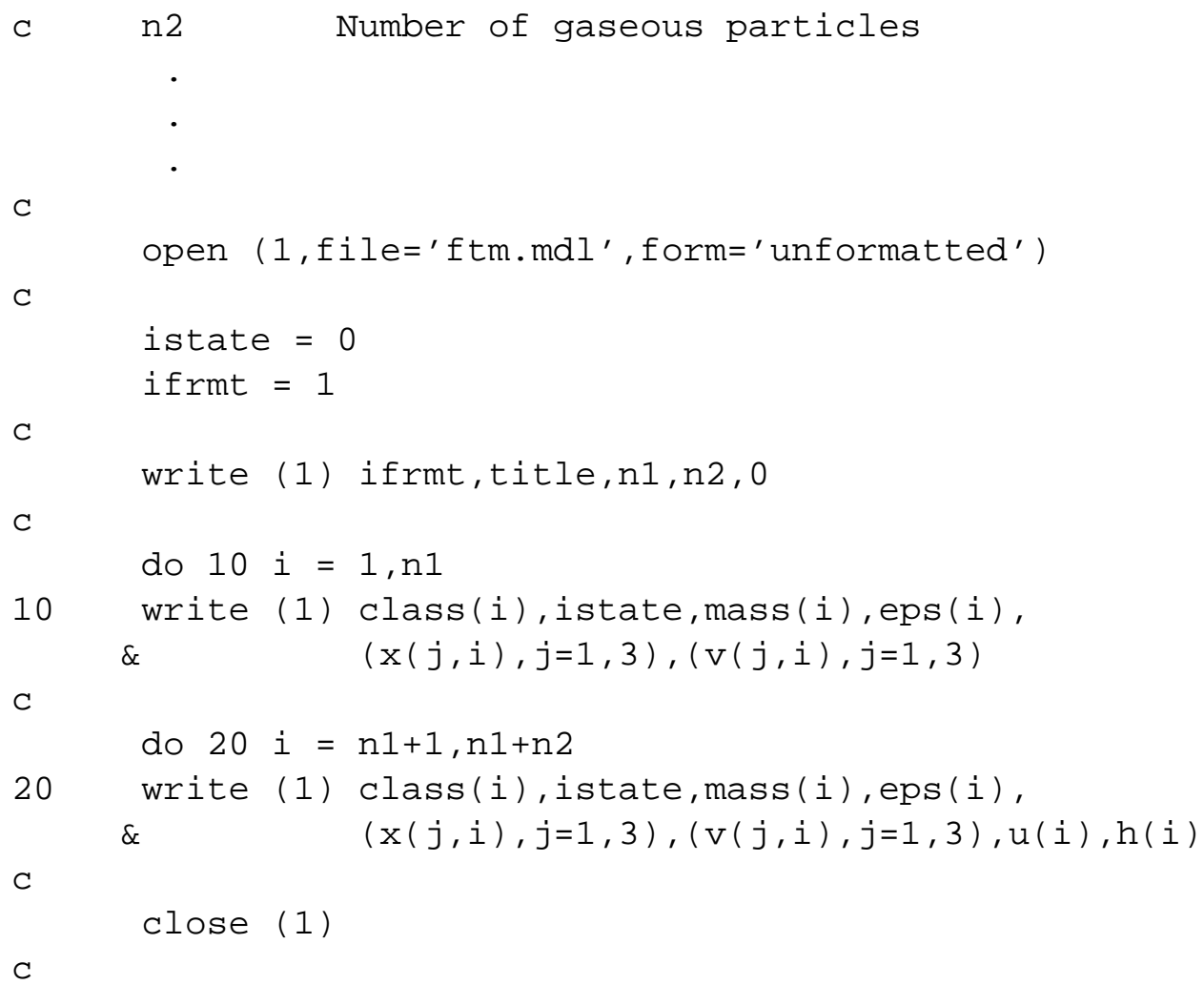

C

Note: For an example on how to access the dump file (FTM.DMP) see the FORTRAN routines FRAME.F, READDMP.F, and READDEF.F in the TOOLS directory.

\section{B.7 Data Structures}

Storage allocations for stellar particles, gaseous particles, and tree cells are defined by compile time parameters, IBLST1, IBLST2, IBLST3, and IELST1, IELST2, and IELST3. The actual locations of particles are defined by run time integer variables, bptr11, bptr12, and eptr11, eptr12. This is shown schematically below:

stellar

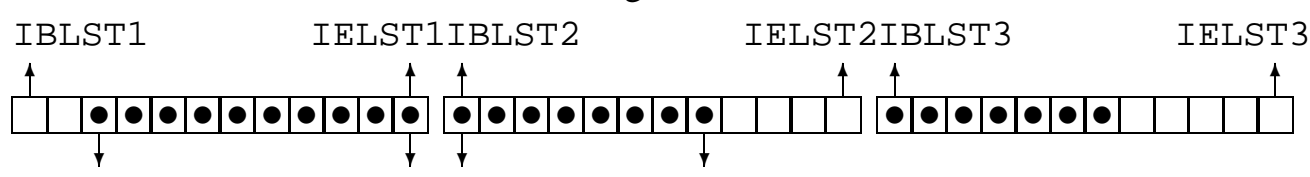
eptr11bptr12 eptr12 


\section{B.8 Examples I}

(I) To access the masses of the stellar particles you might have a loop:

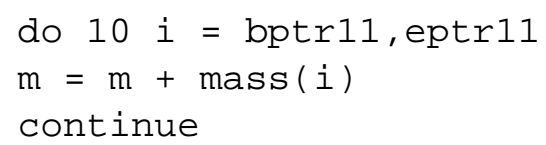

(II) To access the masses of the gaseous particles you might have a loop:

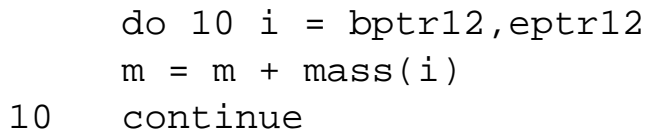

(III) To access the masses of all the particles you might have a loop:

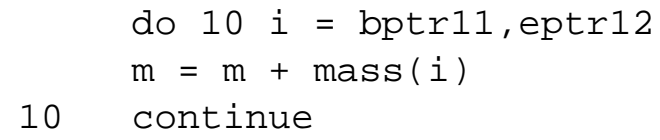

This last example will work even if there are either no stellar or gaseous particles.

For indirect addressing, integer list indx 1 contains the array locations of the particles in the current time-step. The particle addresses are located in the array according to the run time integer variables bptr21, bptr22, and eptr21, eptr22.

\section{B.9 Examples II}

(I) To access the masses of the stellar particles in the current time-step you might have a loop:

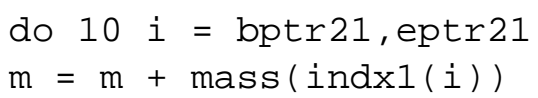

(II) To access the masses of the gaseous particles in the current time-step you might have a loop:

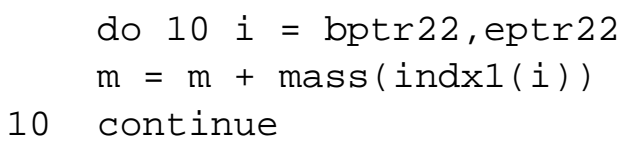

(III) To access the masses of all the particles in the current time-step you might have a loop: 


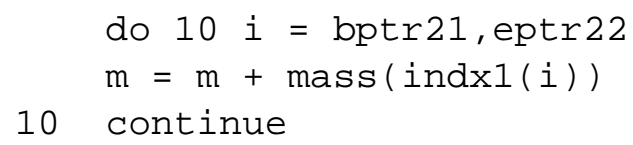

This last example will work even if there are either no stellar or gaseous particles.

NOTE: The first digit on bptr and eptr represents whether its absolute or indirect addressing, and the second digit stellar or gas.

NOTE: The variables bptr13, eptr13, and bptr23, eptr23 are used to define locations for special SPH boundary particles. In general use, when such particles are not present, eptr13 = eptr12 and eptr23 = eptr22. 


\section{B.10 Flow Diagram}

FTM

INITIAL

INTEGRAT

SYNCH

DIAGNOSE

WRTDMP

WRTRST

STATS

TASKTERM

INITIAL

READNML

READMDL

EPSINIT

SOFTINIT

KERNINIT

EXTINIT

TASKORIG

INTGINIT

INTGINIT

LOADINDX

SMOOTH

WARMUP

FREEZE

FORCE

INSERT

TIMESTEP

INTEGRAT

FORCE

TIMESTEP

RESTRCT

BNDRY 1

BNDRY2

FORCE

NEIGHBOR

SETBOX

MAKNTREE

TASK 1

INTERAC1

TASK2

LIMITS

INTERAC2

SPHVAR

SPHSFM entry point

data base initialization

model time integration

time synchronize positions and velocities

Diagnostic output (-> stdout, .txt)

Dump file output (-> .dmp)

Restart file output (-> .rst)

run statistics (-> .txt)

terminate parallel tasks and locks

data base initialization

read input parameters namelist (<-ftm.nml)

read model data file (<- .mdl)

gravitational softening length initialization

gravitational softening interpolation table generation

SPH smoothing kernel interpolation table generation

initialize external potential

create parallel tasks and locks

integration initialization

integration initialization

load all bodies into current list (indx1)

smooth velocity and internal energy fields

assign velocities to disk particles

remove disk particles from integrator

compute total force on current particles (-> stdout, .txt)

insert particles into time stepping data base

compute time-step for current particles

model time integration

compute total force on current particles

compute time-step for current particles

restrict internal energies to positive values

check inner accretion boundary (-> .acc)

check outer removal boundary

compute total force on current particles

Find smoothing lengths and neighborhoods

set tree system box size

construct neighborhood tree

schedule parallel task 1

find immediate neighborhoods

schedule parallel task 2

set tree for neighborhood corrections

neighborhood corrections

compute SPH variables (d, p, c, divv, q)

check for starformation (-> .sfm) 


$\begin{array}{ll}\text { SPHFORCE } & \begin{array}{l}\text { compute hydrodynamic forces } \\ \text { compute marching velocities }\end{array} \\ \text { SPHVEL } & \text { schedule parallel task } 3 \\ \text { TASK3 } & \text { construct gravity tree } \\ \text { MAKGTREE } & \text { compute gravitational forces } \\ \text { GRVFORCE } & \text { compute external forces }\end{array}$




\section{The GRAPE-system in Göttingen (GÖ-GRAPE)}

As a member of the Galactic Dynamics Group at the Department II of the Sternwarte Göttingen, I have installed and maintained the local GRAPE facilities, which have been granted to my research project by the Deutsche Forschungsgemeinschaft (DFG).

The front-end machine is a TATUNG Ultra SPARC system COMPstation U Series (TWS-9320) with an UltraSparc II $233 \mathrm{MHz}$ processor (single CPU). The machine runs currently under the operating system Solaris 2.6. All software related to the GRAPE has been compiled with the OSF compiler gcc/g77 obtained from the GNU Project.

The SBus-to-VMEbus adapter, connecting the front-end and the GRAPE board is the Performance TeChnOlogies InC. model PT-SBS915. The Grape-board is the Grape3 Af version. It uses the 8-chip PCB board (without broadcast mode support). 


\section{Acknowledgements}

I have had the opportunity to interact and to work with many different people during all those years, for which I am very grateful. It is a pleasure to thank all of you for such a great time. Furthermore, I had the priviledge and pleasure to work in the old historical observatory in Göttingen, whose inspiring atmosphere is quite unique.

I would like to thank Prof. Klaus J. Fricke for his permanent interest in my work and for supporting me - in many different ways - during all the years. I would also like to thank my supervisor Prof. Evangelie Athanassoula, who monitored all my work and carefully read and provided most valuable comments on the manuscripts presented in this thesis. I owe her lots of gratitude for many fruitful and stimulating discussions and for her excellent support. Furthermore, I am greatly indebted to Prof. Clayton Heller for providing his $N$-body/SPH code and for making this thesis possible in the first place. With his enthusiasm, inspiration and great efforts to explain things clearly and simply, he has been a perfect teacher to me. I would also like to thank Prof. Albert Bosma for many fruitful discussions and for giving valuable aid with the proposals.

I am indebted to all members of the Department II at the Sternwarte Göttingen. Especially, I would like to thank Polichronis Papaderos, Matthias Grott, Kai Noeske and Jörg Huber for many coffee-break discussions. The latter would not have been possible without the support of Thomas Lilly - thanks for the coffee. Special thanks also to Dagmar Krefting for valuable discussions about nonlinear dynamics. I would also like to thank the VW junior research group - Bodo Ziegler, Klaus Jäger, Alexander Fritz and Asmus Böhm - for supporting me in various aspects.

I had the great opportunity to visit and work at the Observatoire de Marseille and would like to thank all members of the institute and particularly the Galactic Dynamics Group for their great hospitality and cordiality. I am deeply indebted especially to Annie Lavall: without her dedication and care, my visits in Marseille would not have taken place so smoothly at all.

My special thanks to Angelos Misiriotis for many discussions about bars in galaxies and in Marseille, the life, the universe and everything. Information:

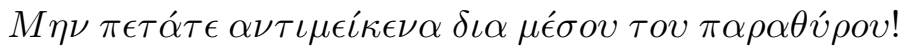

Furthermore, I am deeply grateful to Luz-Maria Montoya, Leobardo Alfaro-Avena, Jorge Villa-Vargas, Carlos Barbera and Jorge Iglesias for many nice days and evenings in Marseille.

Most of the work presented in this thesis is based on numerical computations, which requires stable computers and networks and thus many people to ensure both. Without the great support of Peter Weilbacher at the Sternwarte Göttingen and Jean-Charles Lambert and Philippe Balard at the Observatoire de Marseille this work would not have been possible. Planting and harvesting Grapes is not an easy task. Thus I would like to thank Rainer 
Spurzem and Christian Theis for all their efforts and support on getting the GÖ-Grape system up and running.

Mein ganz besonderer Dank gilt meinen Eltern, Ingrid und Robert Berentzen, die mir das Studium ermöglicht haben und für den familiären Rückhalt, den sie mir in allen Lebenslagen geboten haben. Des weiteren möchte ich mich bei Henriette Pleuß für die täglichen Ablenkungen und ihre vielseite Unterstützung - besonders während der Schlußphase dieser Arbeit - herzlichst bedanken.

This research has been funded by the Deutsche Forschungsgemeinschaft (DFG) under grant Fr 325/48-1, 48-2 and 48-3 and by the Volkswagen-Stiftung under grant I/76520. Furthermore, I thank the DFG for funding the GRAPE facilities at the Sternwarte Göttingen. Finally, I would like to thank the Observatoire de Marseille and the Institute of Theoretical Physics and Astrophysics in Kiel for giving access to the local GRAPE-facilities for part of the simulations presented in this work. 


\section{E. Lebenslauf}

\section{Persönliche Daten}

Name:

geboren am/in:

Staatsbürgerschaft:

Familienstand:

\section{Bildungsweg}

1977-1981

1981-1983

1983-1990

Juni 1990

\section{Grundwehrdienst}

Juli 1990 - Juni 1991

\section{Wissenschaftlicher Werdegang}

seit Oktober 1991

14. Oktober 1993

07. Februar 1997

seit Februar 1997

\section{Fortbildungen}

1997-1998

\section{Berufstätigkeit}

1994-1997

1997-1999

1999-2003
Ingo Berentzen

26.08.1970 in Göttingen

Deutsch

Ledig

Grundschule in Göttingen

Orientierungsstufe in Göttingen

Gymnasium in Göttingen

Abitur

in Rothwesten und Göttingen

immatrikuliert an der

Georg-August-Universität Göttingen

Diplomvorprüfung Physik

Diplom Physik

Promotionsstudium Physik

Netzwerk- und Computeradministration

Wissenschaftliche Hilfskraft an der

Universitäts-Sternwarte Göttingen

Netzwerkadministrator/Wissenschaftlicher Mitarbeiter, Universitäts-Sternwarte Göttingen

Wissenschaftlicher Mitarbeiter,

Universitäts-Sternwarte Göttingen 
